Portland State University

PDXScholar

$6-2-2020$

\title{
Factors Affecting Clinical Research Enrollment Among Individuals with Alzheimer's Disease and Related Dementias
}

Nicole Grace Bouranis

Portland State University

Follow this and additional works at: https://pdxscholar.library.pdx.edu/open_access_etds

Part of the Public Health Commons

Let us know how access to this document benefits you.

Recommended Citation

Bouranis, Nicole Grace, "Factors Affecting Clinical Research Enrollment Among Individuals with Alzheimer's Disease and Related Dementias" (2020). Dissertations and Theses. Paper 5465.

https://doi.org/10.15760/etd.7337

This Dissertation is brought to you for free and open access. It has been accepted for inclusion in Dissertations and Theses by an authorized administrator of PDXScholar. Please contact us if we can make this document more accessible: pdxscholar@pdx.edu. 
Factors Affecting Clinical Research Enrollment Among Individuals with Alzheimer's Disease and Related Dementias

by

Nicole Grace Bouranis

A dissertation submitted in partial fulfillment of the requirements for the degree of

Doctor of Philosophy

in

Health Systems and Policy

Dissertation Committee:

Sherril B. Gelmon, Chair

Allison Lindauer

Dawn Richardson

Elizabeth Needham Waddell

Hyeyoung Woo

Portland State University

2020 
(C) 2020 Nicole Grace Bouranis 


\begin{abstract}
The need to increase ADRD research participation has become more pressing as the prevalence of ADRD increases. Nearly 70,000 Oregonians and 7 million people in the United States live with ADRD, and this number is expected to increase by $200 \%$ by 2050 without identification of an intervention to halt its increase. Developing mechanisms for effective care and treatment depends on implementing research with numerous participants. Historically, ADRD research programs have had difficulty recruiting and enrolling individuals into studies for a variety of reasons. Given low recruitment rates, the interest in researching and evaluating effective strategies to recruit specifically for ADRD research has increased.
\end{abstract}

This dissertation asks "What are the factors affecting clinical research enrollment among individuals with ADRD?" This study has three specific aims: Aim 1: Identify organizational, system, and policy factors that impede or enhance clinical research enrollment among people with ADRD within the Portland metro area; Aim 2: Describe personal factors that persuade or dissuade individuals with ADRD from enrolling in clinical research; and Aim 3: Develop motivational strategies and policy recommendations based on the findings from Aims 1 and 2.

Semi-structured interviews were conducted in two phases with ADRD clinicians, researchers, advocates, people living with ADRD, and caregivers. A community advisory board $(\mathrm{CAB})$ comprised of clinicians, researchers, advocates, people with dementia, family caregivers, and older African Americans was established for this project. The 
$\mathrm{CAB}$ helped develop recruitment and dissemination strategies, modify protocols, and interpret findings. All protocols were approved by the Portland State University Institutional Review Board.

Nine clinicians, researchers, and advocates who were employees or volunteers with health, research, and advocacy organizations of varying sizes and structures were interviewed in Phase 1. Several findings emerged from the interviews. Federal policy attempts to streamline research studies have not resulted in the intended outcomes at the local level. People with ADRD and caregivers have a lack of awareness of research opportunities. In some cases, this was due to lack of provider knowledge; in others, it was due to organizational directives to not discuss this topic or a provider's personal preference. Research opportunities are not shared in places where people with ADRD and their caregivers are likely to congregate. Interviewees were often hesitant to join community collaborations or formal partnerships, and many were weary of including people with ADRD in their advisory boards.

In Phase 2, twelve dyads of people with ADRD and their caregivers $(n=24)$ were interviewed. Many interviewees were unaware of dementia studies, which was surprising as many interviewees were recruited from organizations with rich knowledge of research opportunities. One's perceived value of ADRD research did not increase their likelihood of joining a study. Many caregivers either did not have or did not want to use limited free time to be a study partner; however, several caregivers noted that they would participate in a study if provided some sort of caregiver benefit to ease their burden, such as support, 
education, or respite. A substantial number of interviewees indicated they avoid research because it involves taking study drugs.

The Robert Wood Johnson Foundation's Culture of Health Action Framework was used to conceptualize motivation strategies and reflect elements that describe research participation among people with dementia. Six strategies were identified to improve dementia clinical research participation: 1) Identify and promote local champions for ADRD clinical research participation; 2) Promote policies and processes that incentive cross-sector collaboration; 3) Recognize caregivers as full research participants; 4) Include people with ADRD and caregivers in the research design process; 5) Offer alternative options to reduce participation burden; 6) Evaluate and improve relationships between healthcare/research staff and patients/participants. These strategies can be used in conjunction with the Culture of Health Action Framework as a roadmap to form organization-community partnerships, facilitate motivation and empowerment, give decision-making power to people with ADRD and promote a local culture of research.

This study contributed to the literature by providing contextually relevant factors affecting clinical research participation as well as providing recommendations to improve recruitment. Further, this study successfully demonstrated that including people with dementia and caregivers on an advisory board can be effective, and the use of a CAB improves the study design and interpretation of findings. Through the findings and conclusions of this study, the potential exists to increase research participation in Portland, OR, and can be contextually relevant in other geographic areas where ADRD research is conducted. 


\section{ACKNOWLEDGEMENTS}

The following people and organizations have made a significant impact throughout my dissertation process. Sherril Gelmon, thank you for believing in me from Day One, agreeing to chair my dissertation, and for always pushing me outside of my comfort zone. Allison Lindauer, thank you for taking a second appointment at the OHSU-PSU School of Public Health so you could join my committee, and for sharing your caregiving and ADRD clinical research wisdoms with me. Dawn Richardson, thank you for sharing your extensive community engaged research knowledge with me, for being a part of the Bouranis Community Advisory Board, and for thoughtfully helping me work through my method and research design questions. Elizabeth Needham Waddell, thank you for providing such detailed feedback and encouraging me to find my voice. Hyeyoung Woo, thank you for taking on the role of Graduate School Representative and assuring that my work meets the school's requirements.

To the Bouranis Community Advisory Board members, thank you for taking time out of your busy schedules to share your expertise that I would not have been able to find elsewhere. Because of all of you, this study is better than I could have thought possible. To the PreSERVE Coalition for Black/African American Memory and Brain Health, I am so grateful for the relationships I have made and the knowledge I have received. To my classmates, Dr. Shauna Petchel, Chad Johnson, Kelly Coates, and Liz Walker, thank you for being there for the past few years for all the highs and lows that only HS\&P doctoral students can understand. To the employees of 2 Stroke Coffee in St. Johns and Starbucks 
in Goose Hollow, thank you for providing me a space to write for hours on end. To my husband, Brendan Doran: words cannot describe how grateful I am for you. And to Papa Mike and Uncle Mike, thank you for inspiring me to do this work.

This study was supported, in part, by Oregon citizens through the Alzheimer's Disease Research Fund of the Oregon Charitable Checkoff Program. This fund is administered by the Oregon Partnership for Alzheimer's Research. 


\section{TABLE OF CONTENTS}

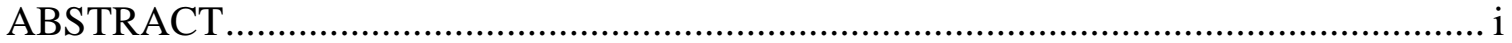

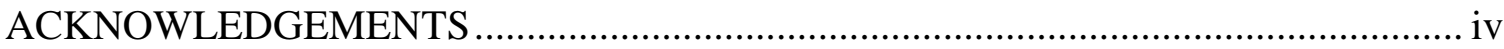

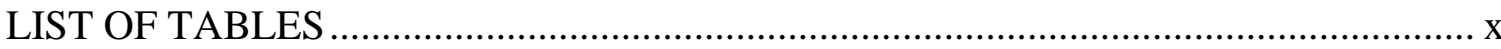

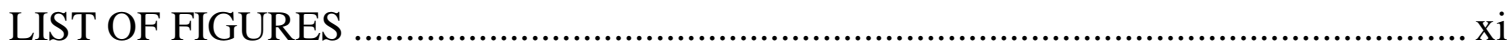

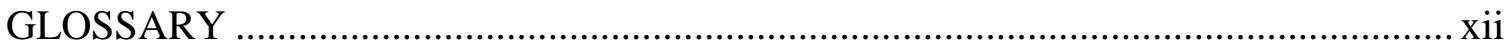

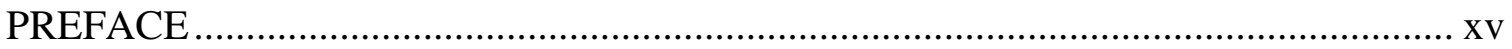

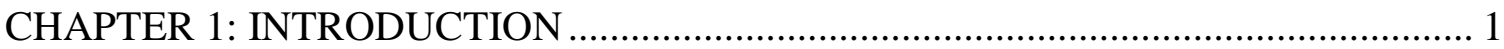

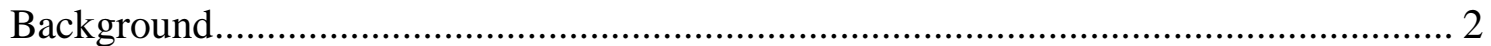

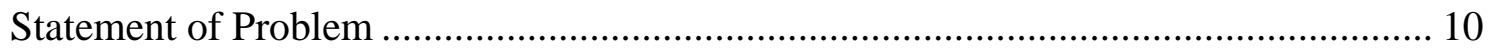

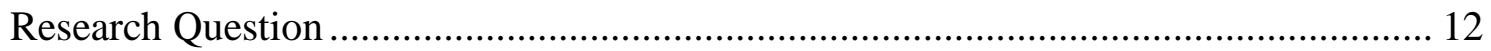

Conceptual and Theoretical Frameworks ........................................................ 12

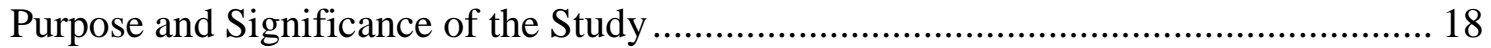

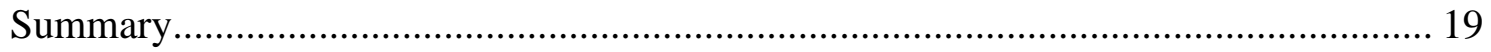

CHAPTER 2: REVIEW OF THE LITERATURE ................................................... 20

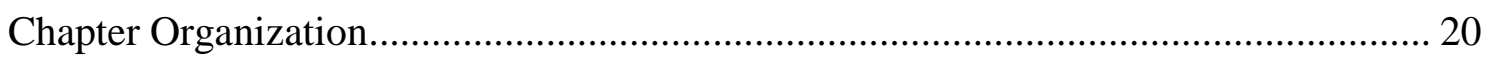

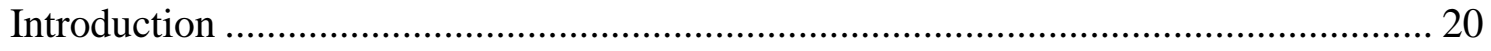

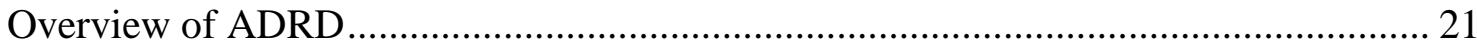

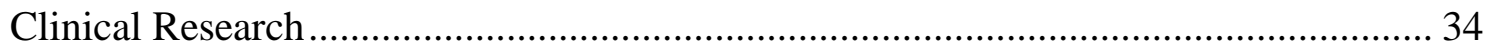

Barriers, Facilitators, and Strategies Influencing Clinical Research Participation ........ 44

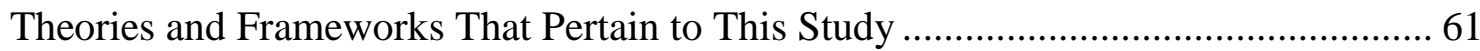

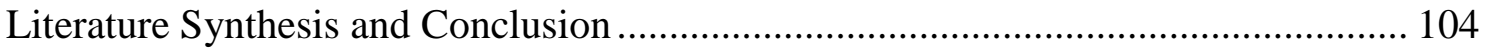

CHAPTER 3: RESEARCH DESIGN AND METHODOLOGY ................................ 106 


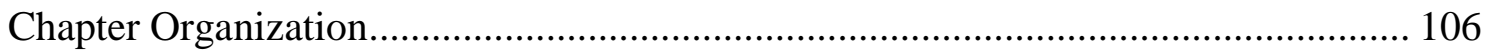

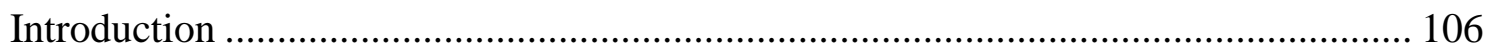

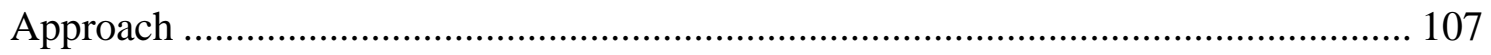

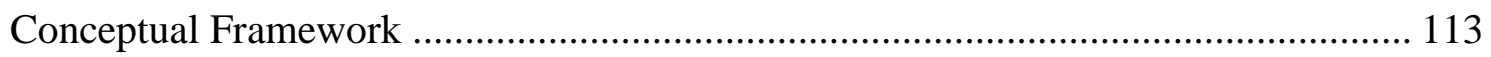

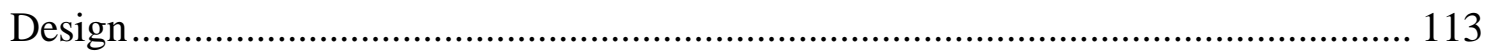

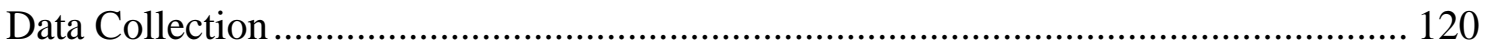

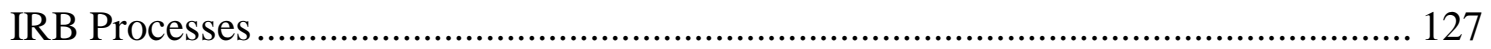

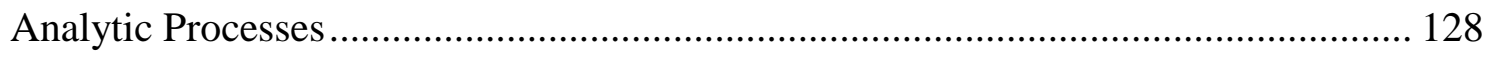

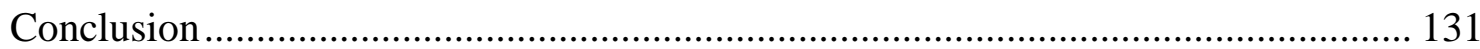

CHAPTER 4: ORGANIZATIONAL, SYSTEM, AND POLICY FACTORS

AFFECTING DEMENTIA CLINICAL RESEARCH PARTICIPATION .................... 132

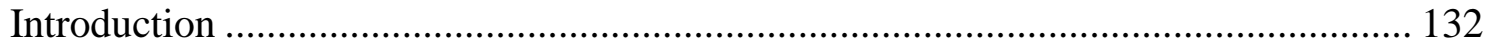

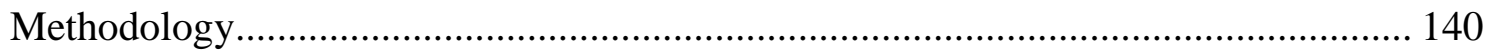

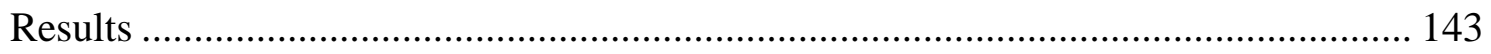

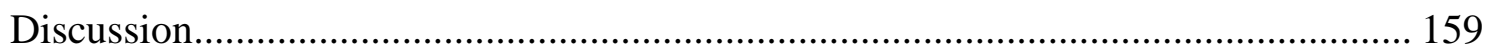

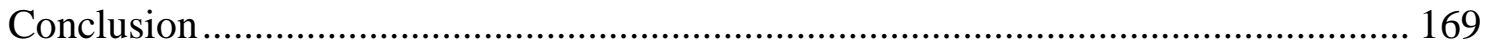

CHAPTER 5: PERSPECTIVES OF PEOPLE WITH DEMENTIA AND CAREGIVERS ABOUT DEMENTIA CLINICAL RESEARCH .................................................... 175

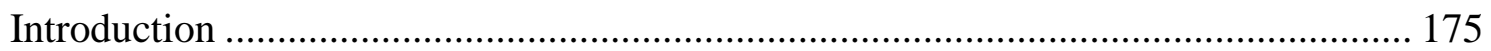

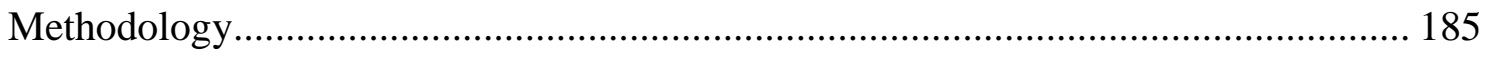

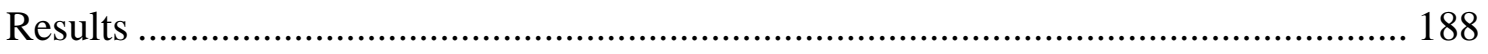


Integration of Findings and Theories

Discussion 208

Conclusion 214

CHAPTER 6: RECOMMENDATIONS TO IMPROVE LOCAL DEMENTIA

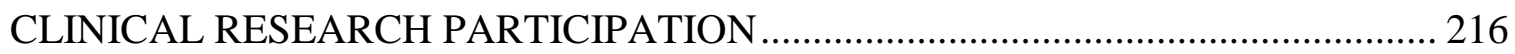

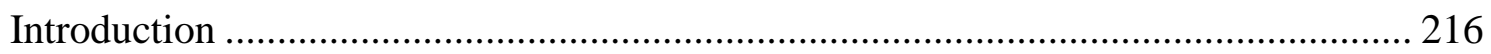

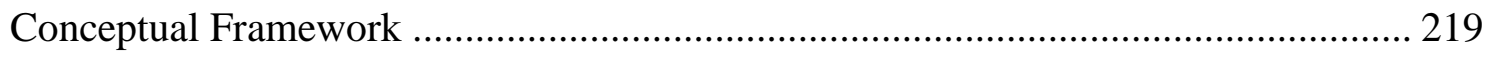

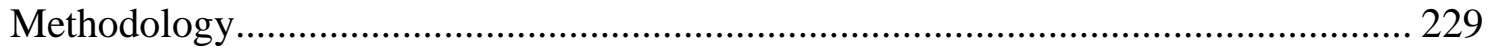

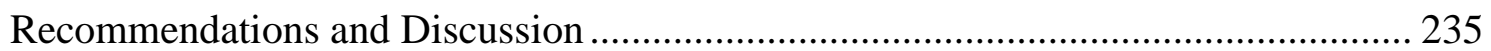

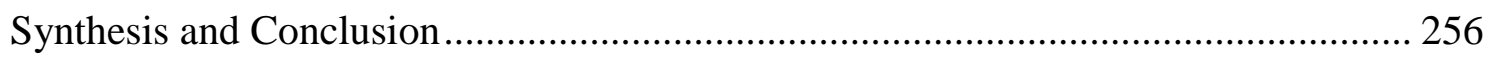

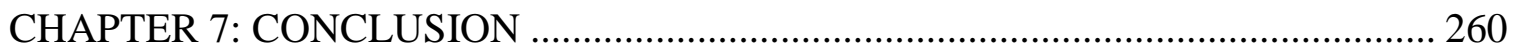

Overview of the Study Purpose and Summary of Findings ......................................... 260

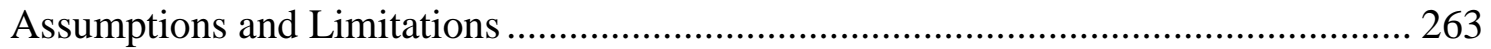

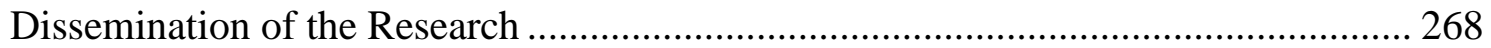

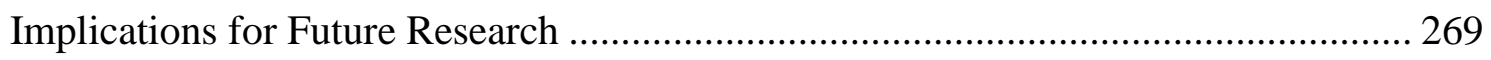

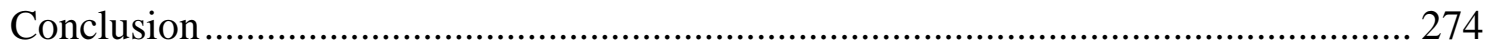

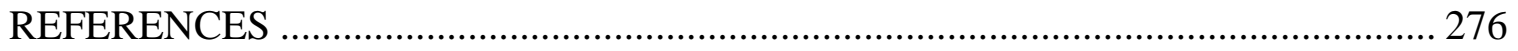

APPENDIX A: CAB WELCOME AND EXPECTATIONS ………………………...... 305

APPENDIX B: FIRST CAB MEETING AGENDA AND NOTES …………………. 310

APPENDIX C: SECOND CAB MEETING AGENDA AND NOTES ………………... 314

APPENDIX D: THIRD CAB MEETING AGENDA AND NOTES …………………... 318

APPENDIX E: RECRUITMENT EMAIL ............................................................ 322

APPENDIX F: RECRUITMENT FLYER …………………................................. 323

viii 
APPENDIX G: SCREENING PHONE SCRIPT 324

APPENDIX H: KEY INFORMANT CONSENT FORM ........................................... 326

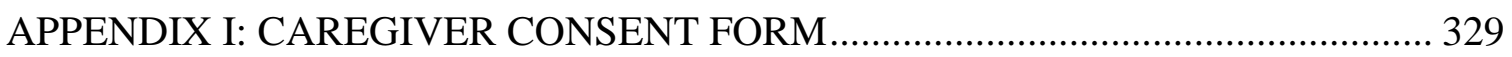

APPENDIX J: PERSON WITH ADRD CONSENT FORM .......................................... 333

APPENDIX K: KEY INFORMANT INTERVIEW PROTOCOL ………………….... 337

APPENDIX L: CAREGIVER INTERVIEW PROTOCOL ........................................... 339

APPENDIX M: PERSON WITH ADRD INTERVIEW PROTOCOL............................ 341

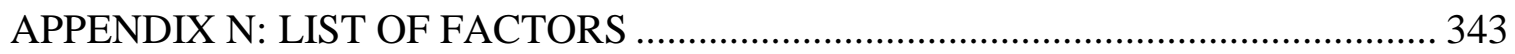




\section{LIST OF TABLES}

Table 2.1 Overview of Clinical Trial Phases.............................................35

Table 2.2 Ethical Guidelines in Clinical Research.................................40

Table 2.3 Operationalization of the Culture of Health Action Framework.................101

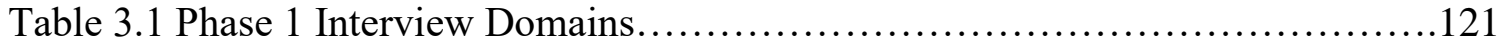

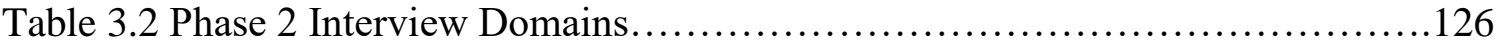

Table 4.1 Factors Affecting ADRD Research Participation..........................133

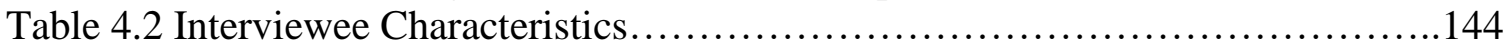

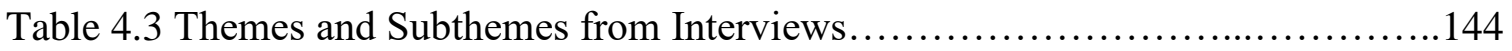

Table 4.4 Themes in Relation to Organizational Factors.............................160

Table 4.5 Themes in Relation to System Level Factors.................................163

Table 4.6 Themes in Relation to Policy Level Factors................................ 166

Table 5.1 Theory Summary ................................................... 184

Table 5.2 Characteristics of Interviewees with ADRD and Caregivers..................189

Table 5.3 Themes and Subthemes from Interviews................................. 190

Table 5.4 Interviewees Transaction Costs of ADRD Research Participation.............204

Table 6.1 Characteristics of Interviewees...........................................234

Table 6.2 Characteristics of Interviewees with ADRD and Caregivers ..................235

Table 6.3 Culture of Health Action Areas and Corresponding Recommendations.......236 


\section{LIST OF FIGURES}

Figure 1.1: The Culture of Health Action Framework................................ 13

Figure 2.1: The Five Basic Parts of the Organization..............................65

Figure 2.2: Policy Feedback for Mass Publics...................................... 71

Figure 2.3 Multi-Level Process Feedback...........................................74

Figure 2.4 The Culture of Health Action Framework.................................90

Figure 3.1 Degrees of Community Engagement.................................. 111

Figure 3.2 Dissertation Aims...................................................118

Figure 5.1 Policy Feedback for Mass Publics: How Policy Affects Civic Engagement.179

Figure 6.1 Culture of Health Action Framework...................................220 


\section{GLOSSARY}

AAIC Alzheimer's Association International Conference

AARP Association of American Retired Persons

AD Alzheimer's disease

ADL Activities of daily living

ADRC Alzheimer's Disease Research Center

ADRD Alzheimer's disease and related dementias

AL Assisted living

APOE Apolipoprotein E

CAB Community Advisory Board

Caregiver A person, typically a family member or friend, who provides unpaid caregiving for a person with ADRD

CBC Community-based care

CBPR Community Based Participatory Research

CCO Coordinated Care Organization

CDC Centers for Disease Control and Prevention

CITI Collaborative Institutional Training Initiative

COC Certificates of confidentiality

$\mathrm{CoH} \quad$ Culture of Health

CRT Critical Race Theory

CVD Cardiovascular disease 
DeNDRoN Dementias and Neurodegenerative Research Network

DLB Dementia with Lewy bodies

EHR Electronic Health Record

FDA $\quad$ Food \& Drug Administration

FTD Frontotemporal dementia

GCP Good Clinical Practice

HHS Department of Health and Human Services

HIV/AIDS Human Immunodeficiency Virus/Acquired Immunodeficiency Syndrome

IADL Independent activities of daily living

ID Intellectual disability

IRB Institutional Review Board

LEAD Leaders Engaged in Alzheimer's disease

LTSS Long-term supports and services

MC Memory care

MCI Mild cognitive impairment

MMSE Mini-mental state exam

MoCA Montreal Cognitive Assessment

MRI Magnetic resonance imaging

NAC National Alliance for Caregiving

NIA National Institute on Aging

$\mathrm{NIH} \quad$ National Institutes of Health

OHRP Office of Human Research Protections 


$\begin{array}{ll}\text { OHSU } & \text { Oregon Health \& Science University } \\ \text { ORCID } & \text { Open Researcher and Contributor Identification } \\ \text { PCORI } & \text { Patient-Centered Outcomes Research Institute } \\ \text { PCPCH } & \text { Patient Centered Primary Care Home } \\ \text { PCMH } & \text { Patient Centered Medical Home } \\ \text { PET } & \text { Positron emission tomography } \\ \text { PFT } & \text { Policy Feedback Theory } \\ \text { PreSERVE } & \text { PreSERVE Coalition for Black/African American Memory and Brain } \\ & \text { Health } \\ \text { PSU } & \text { Portland State University } \\ \text { RC } & \text { Residential care } \\ \text { REACH } & \text { Racial and Ethnic Approaches to Community Health } \\ \text { WEPIC } & \text { West Philadelphia Improvement Corps } \\ \text { WHO } & \text { World Health Organization }\end{array}$




\section{PREFACE}

In preparing this dissertation, Chapters 4,5 , and 6 were written as independent, freestanding papers. After the successful defense of this dissertation, these three chapters will be edited and submitted independently for publication. Due to this formatting, there is some redundancy in these chapters with other content in the dissertation. 


\section{CHAPTER 1: INTRODUCTION}

Nearly seven million people in the United States live with Alzheimer's disease and related dementias (ADRD), costing the nation over 290 billion dollars per year in direct health and long-term care costs (National Institute on Aging [NIA], 2018; Alzheimer's Association, 2019a). Given the rapidly aging population, this number will exceed 8 million by 2025, and 18 million by 2050 (Alzheimer's Association, 2019a). ADRD is a neurodegenerative, progressive, terminal disease and is the only top ten cause of death without a treatment, prevention, or cure (NIA, 2016). Many medical professionals, policymakers, and those affected by the disease agree that rapidly increasing ADRD prevalence rates (and subsequent rising costs) coupled with the lack of clinical solutions, is creating a medical, economic, and emotional crisis (Gates, 2017; Collins et al., 2018; NIA, 2019; Alzheimer's Association, 2019a; Gates, 2019).

To combat this crisis, the U.S. Congress allocated 2.8 billion dollars for ADRD research in 2019 with the goal of finding solutions for prevention or treatment by 2025 (Alzheimer's Association, 2019b). Advancements in ADRD research are in part contingent on research participation by large numbers of participants (Rollin-Sillaire et al., 2013; Law, Russ, \& Connolly, 2014, Grill \& Galvin, 2014; Black et al., 2018; NIA, 2018; Gates, 2019, Gilmore-Bykovskyi et al., 2019). To effectively utilize this historic funding, efforts to increase participation in clinical trials are needed. The National Institutes of Health (NIH) recognized this need and has allocated funding for studies that focus on ADRD clinical research recruitment, retention, and diversity (NIH, 2018). 
Additionally, the NIA released a national strategy for improving research participation in ADRD studies in October 2018 (NIA, 2018).

The national strategy provides strong recommendations; however, two gaps persist. First, the national strategy is not a comprehensive list of barriers and facilitators; the NIA recommend continued research on identifying more factors affecting participation (NIA, 2018). Second, these recommendations were developed by working groups of ADRD clinicians, researchers, and advocates; the voice of individuals with ADRD was not included. Including people with ADRD in research. However, including the voice of people with ADRD is one of the recommendations from this strategy (NIA, 2018). Additionally, there have been calls from academics and advocates to do so (Iliffe, McGrath, \& Mitchell, 2013; McGrath, 2017; Kremer, 2017; Gitlin, Maslow, \& Khillan, 2018; Gilmore-Bykovskyi et al., 2019).

This chapter begins with a description of ADRD and the challenges to recruit clinical trial participants. This is followed by the problem statement, the research question and an outline of the conceptual and theoretical frameworks. The chapter concludes with the purpose and significance of the study and a succinct summary, setting up the overall research for the dissertation.

\section{Background}

Before articulating the reasons why efforts to improve ADRD clinical research recruitment are needed, a brief overview of ADRD is provided here. This is followed by a discussion on those affected by ADRD, such as the person diagnosed, their caregivers, 
friends, family, and the health and long-term supports and services (LTSS) sectors. Lastly, challenges to recruiting clinical research participation are discussed.

\section{Alzheimer's disease and related dementias (ADRD)}

Dementia is a syndrome resulting in a loss of cognitive and behavioral functions. There are several forms of dementia with varying symptoms; however, the primary diagnostic signs are the loss of core mental and brain functions (such as language skills, problem-solving, and cognition) and the ability to perform everyday activities (NIA, 2016).

The most common form of dementia is Alzheimer's disease (AD), which accounts for $60-80 \%$ of all cases of dementia (NIA, 2016). AD is an "irreversible, progressive brain disorder that slowly destroys memory and thinking skills and, eventually, the ability to carry out the simplest tasks" (NIA, 2017). Common symptoms include loss of language, spatial issues, impaired decision-making, behavioral changes, and a decline in every day abilities (NIA, 2017). Though not a form of dementia, mild cognitive impairment (MCI) is included in discussions of dementia and memory loss (Roberts \& Knopman, 2013). MCI results in a less severe case of memory loss and does not result in behavior or personality changes, however, individuals with MCI are a significantly higher risk of developing $\mathrm{AD}$ as compared to those with normal cognitive function (NIA, 2017).

In addition to $\mathrm{AD}$, there are several other forms of neurodegenerative, progressive dementias that are included in the definition of ADRD. Dementia with Lewy bodies dementia (DLB) results in a decline of thinking, reasoning, and independent function 
(Zweig \& Galvin, 2014; Gomperts, 2018). People with DLB exhibit more gait and movement symptoms as well as more extreme behavioral issues, such as delusions and hallucinations, in its early stages as compared to those with AD (Zweig \& Galvin, 2014; Gomperts, 2018). Another form, frontotemporal dementia (FTD), presents with significant behavioral changes and more muscle deterioration, including speech and language functioning, and loss of spatial awareness (Snowden, Neary \& Mann, 2002; Hernandez, Fernandez, Tarraga, Boada, \& Ruiz, 2017). People with FTD also experience memory loss early on, but it becomes more prominent in later stages as compared to $\mathrm{AD}$ or DLB (Snowden et al., 2002; Hernandez et al., 2017). Vascular dementia is caused by a loss of blood flow to the brain, most frequently due to strokes, with symptoms extremely similar to AD (Love, 2005; Kalaria, Alinyemi, \& Ihara, 2016). However, this dementia can be prevented or halted provided there is a cessation of the vascular events (Love, 2005; Kalaria, et al., 2016). Lastly, when individuals experience symptoms characteristic of more than one type of dementia, they are considered to have mixed dementia (Schneider, Arvanitakis, Bang, \& Bennett, 2007).

Virtually everyone with ADRD will require LTSS due to the disease's debilitating and progressive symptoms. Long-term supports and services include a variety of supports, such as in-home respite care, day centers, community-based residential care, and nursing homes. ADRD significantly contributes to the 339 billion dollars in annual national LTSS costs, two-thirds of which are covered by Medicaid and Medicare (Colello \& Talaga, 2015). These high costs are concerning to many individuals and families as they plan for, or suddenly find themselves needing, LTSS. According to federal policies, 
Medicare can only be used for 100 days of nursing home care, which is typically reserved for severe cases of ADRD (Kaiser Family Foundation, 2016). Depending on the scope of state policies, Medicaid may be utilized for LTSS but is contingent on the providers' willingness to accept Medicaid (NORC, 2014). Those who are higher-income may be able to afford private-pay LTSS; however, middle-income individuals find themselves spending down their assets on private-pay LTSS until they become eligible for Medicaid (Hudson, 2014).

Though not the only factor, the high cost of LTSS leads to many families of people with ADRD taking on caregiving roles. Over 20\% of the 34.2 million Americans providing unpaid care to an adult age 50 or older are caring for someone with ADRD (National Alliance for Caregiving \& AARP Public Policy Institute, 2015). These informal caregivers perform more tasks than their non-ADRD caregiving counterparts (National Alliance for Caregiving \& AARP Public Policy Institute, 2015). These tasks include: daily activities such as bathing, dressing, and toileting (referred to as activities of daily living [ADLs]); independent living skills such as managing money, housekeeping, and meal preparation (referred to as instrumental activities of daily living [IADLs]); medical/nursing tasks such as injections and tube feedings; and other key activities such as communicating with healthcare professionals and advocating on the affected person's behalf. Additionally, people of color are significantly less likely to utilize LTSS for a variety of reasons, including stigma within their culture, discrimination from healthcare system, language barriers, access, and the belief that nothing could be done (Chin, Negash, \& Hamilton, 2011; Aranda, 2017). Informal caregivers of people with ADRD 
also suffer from more adverse health outcomes as compared to their non-ADRD caregiving and non-caregiving counterparts, costing the United States an additional 11 billion dollars annually (Kelly, McGarry, Georges, \& Skinner, 2015).

Currently, there is no prevention, treatment, or cure for ADRD. Studies have shown that a healthy lifestyle, being educated, and being socially connected to others lowers the risk of ADRD, but there is no proven prevention that removes the risk completely (Mergenthauler, Lindauer, Dienel, \& Meisel, 2013; Pool et al., 2016; Yates, Ziser, Spector, \& Orrell, 2016). There is one group of drugs that treat cognitive symptoms, but the treatment is a short-term, ranging from six months to a year (Yanniopoulou \& Papegeorgiou, 2013; Alzheimer's Association 2018a). ADRD clinical research has a near $100 \%$ failure rate, partly due to interventions that have not worked; but also because studies have been postponed or terminated due to low enrollment rates (Cummings, Morstorf, \& Zhong, 2014; Grill \& Galvin, 2014; NIA, 2018).

Several organizations, including the Alzheimer's Association, the Leaders Engaged on Alzheimer's Disease (LEAD) Coalition, and the NIA have called for action to increase ADRD clinical research enrollment (Iliffe, McGrath, \& Mitchell, 2013; Kremer, 2017; Alzheimer's Association, 2018; NIA, 2018). However, increasing recruitment requires individuals with ADRD to be willing and able to participate, and many barriers stand in the way.

\section{The challenge of recruiting ADRD clinical research participants}

Many individuals affected by medical conditions, including ADRD, report an interest in joining related clinical research both for potential benefits for themselves and 
opportunities to help others (English, Lebovitz, \& Griffin, 2010; Law et al., 2014; Anderson, Borfitz, \& Getz, 2018). Despite these reports, clinical research participation rates continue to be low (English et al., 2010; Law et al., 2014; Mahon et al., 2016; Anderson et al., 2018; NIA, 2018; Gates, 2019). This is due to a variety of policy, system, organizational, and personal factors. These factors are described in detail in Chapter 2, but a few are described briefly below.

One common sentiment from interested individuals is that they are unaware of how to seek or receive information about opportunities to join clinical research (Sood et al., 2009; Law et al., 2014; Anderson et al., 2018; NIA, 2018). Individuals often expect to hear about studies from their primary care provider and are more likely to participate when these providers inform them due to their trusting relationship. However, individuals may be more likely to hear of opportunities from media advertisements, which are less trusted (Friedman, Foster, Bergeron, Tanner \& Kim., 2015; Anderson et al., 2018).

Second, when designing research studies, investigators adhere to federallydictated ethical and clinical practices that align with policies enforced through their institutional review boards (IRBs). For example, the IRB at Oregon Health \& Sciences University (OHSU), an academic health center which conducts ADRD research, adheres to international regulations on good clinical and ethical practices, federal regulations implemented by the U.S. Department of Health and Human Services, the NIH, the FDA, and Oregon laws and regulations (OHSU, 2017). All research studies are designed to meet these regulations to ensure that risk of harm to research participants is minimized, which may mean that potential study recruits may be deemed ineligible due to 
comorbidities or prescribed contraindicated medications (Grill \& Galvin, 2014; Adams, Caffrey, \& McKevitt, 2015). This negatively impacts people with ADRD; most are over 65 and are more likely to encounter one of these confounding issues than people under 65 (Rollin-Sillaire et al., 2013; Grill \& Galvin, 2014; Law et al., 2015). Additionally, many ADRD clinical research studies require the participation of study partners to ensure the person with ADRD attends appointments and adheres to study protocols, a role typically taken on by the primary informal caregiver of the person with ADRD (Rollin-Sillaire et al., 2013; Black, Taylor, Rabins \& Karlawish, 2018). Study partners attend clinical research appointments and ensure that the person with ADRD adheres to study requirements (Rollin-Sillaire et al., 2013; Black et al., 2018). Though study partners are necessary to ensure the safety of people with ADRD and the validity of the study, this requirement further limits the pool of eligible participants to those with a caregiver who is willing and able to act as a study partner (Karlawish et al., 2008; Watson, Ryan, Silverberg, Cahan, \& Bernard, 2014; Grill \& Galvin, 2014; Cary, Rubright, Grill \& Karlawish. 2015; Black et al., 2018).

Furthermore, study sponsors, who fund the research and may be from the government, nonprofit, or private sectors, may have a role in designing the studies and can require additional stipulations as long as they adhere to ethical and clinical regulations (Califf, 2006; Rollin-Sillaire et al., 2013; Banzi, Camaioni, Tettamanti, Bertele, \& Lucca, 2016; Cummings, Lee, Mortsdorf, Ritter \& Zhong, 2017). For example, despite more than one-third of all ADRD cases occurring in people aged 85 or older, just $8 \%$ of individuals participating in ADRD clinical research between 2000 and 
2015 were over the age of 85 (Banzi et al., 2016). Many studies limit eligibility to individuals 85 or younger, posing a barrier to individuals who may be otherwise healthy and eligible to participate in a clinical research, further limiting the pool of available recruitees. To address this barrier, the NIH revised the guidelines that allowed for arbitrary age exclusion; as of January 2019, clinical research, barring scientific or ethical reasons, can no longer exclude potential recruitees based on age (NIH, 2017a). However, it is unclear how strictly this policy has been interpreted to date, and it may need to be monitored closely to ensure it is being implemented appropriately.

Additionally, there are significant disparities across population groups in clinical research enrollment due to a variety of multi-level barriers. People of color are less likely to participate in clinical research despite being more likely to be diagnosed with ADRD (Danner et al., 2011; Williams, Meisel, Williams \& Morris, 2011; Alzheimer's Association, 2018a; Gilmore-Bykovskyi et al., 2019). This could be due to a variety of reasons, such as distrust in the investigators, a lack of partnerships with local community organizations, advertising by recruiters in venues with high populations of white men, such as academic health centers and universities, or a lack of study partner (Danner, Darnell, \& McGuire, 2011; Mahon et al., 2016, Black et al., 2018).

This background highlights the importance of developing a national, comprehensive ADRD prevention or treatment strategy by 2025 and the necessity of increasing clinical research recruitment efforts. Though many individuals with ADRD may want to participate in clinical research, they may be dissuaded due to lack of awareness; organizational, system, and policy factors that are beyond their control; and 
the lack of availability or interest of their care partner. Further, some individuals with ADRD may not be interested in participating, due to apathy, distrust, or lack of motivation. The national strategy released by the NIA in October 2018 provides recommendations to remove barriers and enhance facilitators to ADRD research participation but recognizes that they do not provide a comprehensive list of strategies and more research needs to be conducted (NIA, 2018). Furthermore, this research was conducted with ADRD clinicians, researchers, advocates, and caregivers. Though they provide important insights, particularly as the policy, system, and organizational level, the voices of those with ADRD were not included. Thus, strategies developed by drawing on the expertise that those living with ADRD offer are missing.

\section{Statement of Problem}

Leaders within the healthcare system recognize that developing partnerships and trusting relationships with community members will improve access to healthy lifestyle options and motivate individuals to take control of their own health (Selby, Beal, \& Frank, 2012; The Robert Wood Johnson Foundation, 2016; Weil, 2016; Tillman, 2017; Gelmon, Bouranis, Sandberg, \& Petchel, 2018). Many health organizations have begun to utilize the Culture of Health Action Framework, established by the Robert Wood Johnson Foundation in 2014, as a reference point and are improving community health outcomes through their multi-sector partnerships (McCullough \& Leider, 2016; The Robert Wood Johnson Foundation, 2018). Clinical research institutions pioneer new interventions for disease prevention and eradication but historically have lagged behind other areas of 
healthcare delivery, such as primary care and mental health, in terms of community outreach and collaboration (English et al., 2010; Adams et al., 2015; Mahon et al., 2016). In recent years, the NIH and the Patient-Centered Outcomes Research Institute (PCORI) have created formative efforts to increase community outreach and collaboration to improve clinical research design, recruitment, and outcomes for ADRD research (PCORI, 2013; NIH, 2018).

Even with the drive to find an ADRD cure or treatment by 2025, as well as the funding to support efforts, investigators continue to struggle to recruit and retain participants into ADRD clinical research (Rollin-Sillaire et al; Law et al., 2014; Watson et al., 2014; Grill \& Galvin, 2014; NIA, 2018; Gates, 2019). Without an adequate number of participants, ADRD clinical research is postponed or terminated, decreasing the chances of reaching the goal of finding an ADRD prevention or treatment strategy by 2025. In recent years, more work has been conducted to determine facilitators and barriers to clinical research subject enrollment, leading to recommendations to improve recruitment (Sood et al., 2009; English et al., 2010; Adams et al, 2015; Carlisle et al., 2015; Mahon et al., 2016; NIA, 2018; Gilmore-Bykovskyi et al., 2019). Some of this literature focuses on ADRD research (Danner et al., 2011; Williams et al., 2011; RollinSillaire et al., 2013; Law et al., 2014; Grill \& Galvin, 2014; Watson et al., 2014; Black et al., 2018; NIA, 2018; Gilmore-Bykovskyi et al., 2019). However, there is little published literature that evaluates if these recommendations have been implemented or improved outcomes. Additionally, there is no published literature that explores barriers, facilitators 
and motivators of ADRD clinical research participation from the lens of people with ADRD.

\section{Research Question}

The research study answered a single question: What factors affect clinical research enrollment among people with ADRD? To answer this overarching question, this study had three aims:

- Aim 1: Identify organizational, system, and policy factors that impede or enhance clinical research enrollment among people with ADRD;

- Aim 2: Describe personal factors that persuade or dissuade individuals with ADRD from enrolling in clinical research; and

- Aim 3: Develop motivational strategies and policy recommendations based on the findings from Aims 1 and 2.

\section{Conceptual and Theoretical Frameworks}

Based on a review of the clinical research and ADRD literature, this research used the Culture of Health Action Framework, developed by the Robert Wood Johnson Foundation in 2014 to encourage collaboration between the health and social sectors (Robert Wood Johnson Foundation, 2016). According to the conceptualization of the framework, health providers will partner with local nonprofits and private organizations; these partnerships then will work with communities to define their needs and the measures that matter most to them to improve population health, wellbeing, and equity 
(Robert Wood Johnson Foundation, 2016). Partners then redesign programs, even if they had been previously successful, to better meet community needs and achieve the Culture of Health Action Framework goals (Weil, 2016). When compared with other communities, those with high-density multi-sector networks supporting health improvement activities will have better health outcomes (McCullough \& Leider, 2016). The Culture of Health Action Framework is a useful way to conceptualize motivation strategies and reflect elements that describe current and future research participation among people with ADRD, because many of the factors affecting clinical research participation can be categorized into the four action areas, as illustrated in Figure 1.1.

\section{Figure 1.1: The Culture of Health Action Framework}

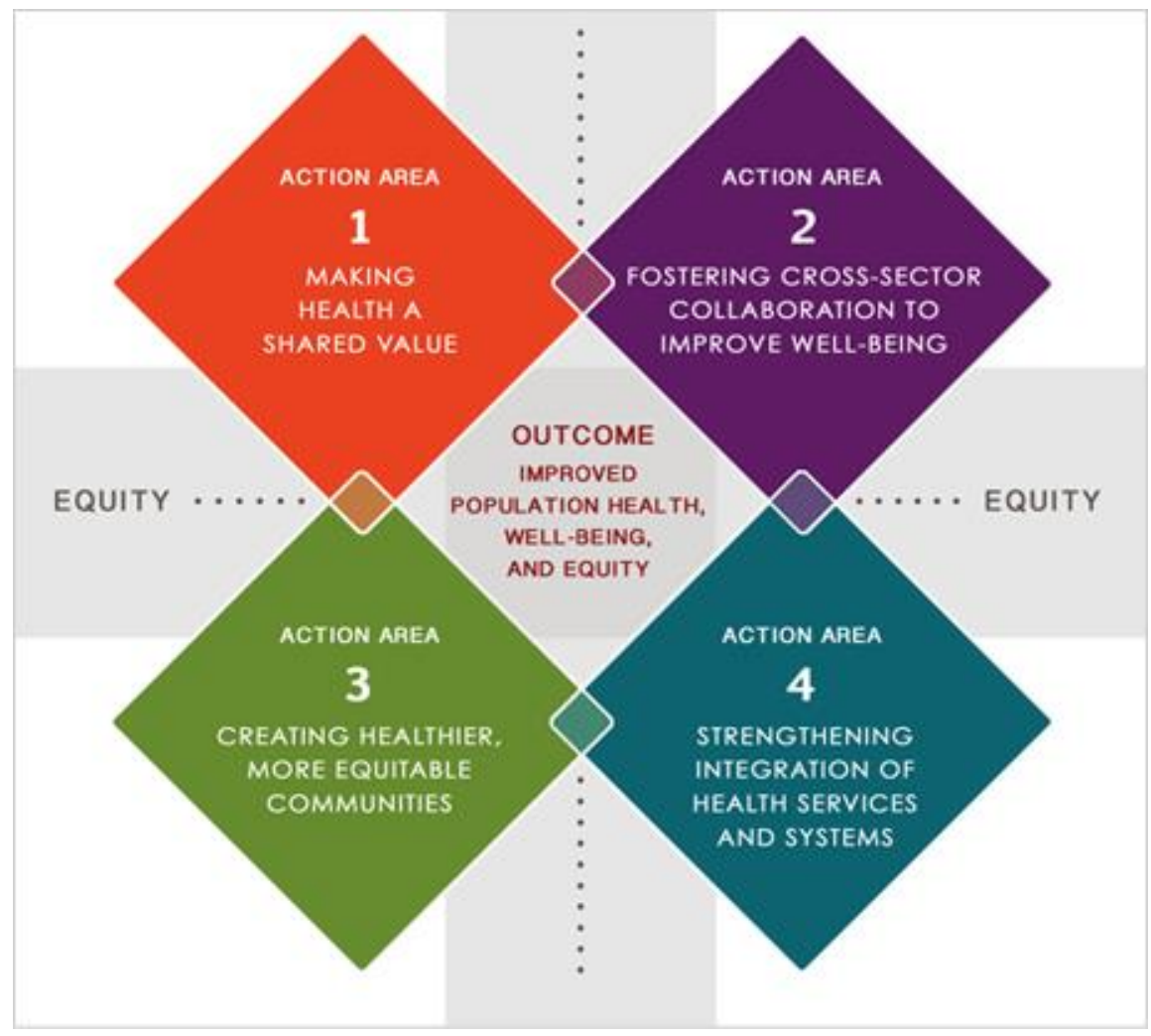


Source: Robert Wood Johnson Foundation, 2016

This framework is rooted in concepts of civic engagement, where community members contribute individually and collectively to better their community. Civic engagement requires knowledge of opportunities to improve the community, values to determine what is best for the community, and motivation to become civically engaged (Chandra et al., 2016).

\section{Civic engagement}

Civic engagement embodies the notion that community members contribute individually and collectively in order to better their communities. Civically engaged individuals work together and develop their knowledge, skills, values, and motivation to make a difference in their community by promoting its quality of life (Ehrlich, 2000). Civic engagement scholars have conducted research to determine ways to foster civic engagement, finding that organizations, governments, and policies influence the community's ability and likelihood to participate in civic engagement activities (Bellah, Madsen, Sullivan, Swidler, \& Tipton, 1991; Denhardt \& Denhardt, 2003; Vigoda-Gadot \& Cohen, 2004, Chandra et al., 2016). Local community initiatives that foster values of education, participation, and community member involvement, such as the collaborative partnerships described by the Culture of Health Action Framework, empower individuals to become stakeholders in their community and increase their civic participation (Bellah et al., 1991; Denhardt \& Denhardt, 2003; Vigoda-Gadot \& Cohen, 2004; Towe et al., 2016). 
Treating community members as stakeholders is imperative to fostering relationships and future collaboration (Sood et al., 2009; Law et al., 2014; Mahon et al., 2016; PCORI, 2017). This is evident in the clinical research sector; current and potential participants cite failure to disseminate research results and updates as a reason for not participating in future opportunities (Law et al, 2014; Mahon et al., 2016). It is also important to recognize historical contexts of previous relationships between community members, policymakers, and organizations. Organization and government leaders often assume that community members will enter into a partnership because it could be beneficial, but due to a history of bad relations or culture, they may not engage (Bellah et al., 1991; Denhardt \& Denhardt, 2003; Danner et al., 2011; Williams et al., 2011). Examples of this dichotomy are evident in the clinical research sector. For example, members of the African American community, who are twice as likely as Caucasians to be diagnosed with ADRD, are less likely to take part in research because of egregious acts such as the Tuskegee Syphilis Study and concerns that researchers do not have the community's best interests in mind (Corbie-Smith, Thomas, Williams, \& Moody-Ayers, 1999; Danner et al., 2011; Brown et al., 2013). However, when clinical research organizations take the time to establish a relationship that builds trust and respect and provide full information about study opportunities in a clear, understandable manner, African Americans are more likely to partner with that research organization (Huang \& Coker, 2010; Williams et al., 2011; Danner et al., 2011; Frew, Archibald, Hixson, \& del Rio, 2011; Inungu, Bender, \& Geiger 2017; NIA, 2018; Gilmore-Bylovskyi et al., 2019). 


\section{Organizational, policy, and methodological theories and approaches}

Aspects of several organizational, policy, and methodological theories are evident in the Culture of Health Action Framework. Organizational theories that pertain to this framework fall within the school of open rational systems (Scott, 1987). Theories in this school emphasize that organizations are means to a predetermined end but recognize the influence the environment has on organizational structures. Contingency theory asserts that the best way to organize is contingent on the nature of the environment (Galbraith, 1973; Mintzberg, 1983). In this case, the nature of the environment refers to the needs of the community and regulations that prohibit or support its needs. Williamson's transaction cost theory suggests that every transaction between two parties is subject to a cost (Williamson, 1975). Restructuring the clinical research organization could further reduce the transaction costs for individuals with ADRD (Califf et al., 2006; Adams et al., 2013; Mahon et al., 2016).

Policy feedback theory (PFT) and social construction theory can explain policy processes. Skocpol's work on PFT illustrates that policies created during one time period affect policies created in future time periods through transformed state capacities and changes in social groups and their political goals and capabilities (Skocpol, 1992). In her Policy Feedback for Mass Publics framework, Mettler highlights how resources and interpretive effects that stem from policies can affect civic predisposition and capabilities, ultimately influencing civic engagement (Mettler, 2002). PFT is influenced by social construction theory, which posits that policies are contingent on policymaker and societal interpretations and values (Schneider, Ingram, \& deLeon, 2014). 
Finally, the Culture of Health Action Framework also utilizes communityengaged research approaches. Using community-engaged research, community members and academics collaborate and develop research and decision-making processes that best address the needs of the community (Wallerstein \& Duran, 2008). Community-engaged research recognizes that despite a common misconception that research is objective, it is not, and it often cannot be. All individuals have different lived experiences, leading to unique perspectives (Habermas, 1963/1974). To establish partnerships, these unique perspectives need to be identified, brought to the table, and discussed. In communityengaged research, professionals recognize and accept the significant power differences between the community and themselves and the effects power differences have on participation behavior. By treating everyone as an equal partner with equal power, knowledge is fully disseminated, empowering the community and mitigating power dynamics (Freire, 1968/1972; Foucault, 1980).

Furthermore, community-engaged research can enhance the study's rigor, relevance, and reach (Balazs \& Morello-Frosch, 2013). Rigor can be enhanced when the community directs researchers to use data collection methods that best addresses their needs, asserting that the research design is appropriate for the questions being asked. Additionally, member-checking, where community members provide feedback of the results that confirm or challenge the investigator's analysis and takeaways from interviews, further enhances scientific rigor by asserting the study's generalizability (Creswell, 2000; Goldblatt, Karneili-Miller, \& Neumann, 2011). Relevance can be enhanced through community identification of research goals that matter most to them; in 
addition, the study's process and findings are disseminated in ways that are most relevant to the community (e.g. pamphlets with clear, concise language). Reach can also be enhanced through dissemination to community members, and can also lead to community testimony to policymakers, potentially resulting in policies beneficial to the community (Balazs \& Morello-Frosch, 2013).

This study's methodology reflects this approach. For instance, this study's process was facilitated by a community advisory board (CAB) comprised of people with ADRD, family caregivers, older African Americans, researchers, clinicians, and advocates. The $\mathrm{CAB}$ assisted with recruitment and interview protocol design, engaged in memberchecking and interpretation of analyses, and brainstormed dissemination strategies. Their collaboration ensured that the research design is rigorous and relevant to people with ADRD by modifying study questions to ask what was most meaningful and pertinent to people with ADRD. The $\mathrm{CAB}$ also developed strategies to ensure that findings and recommendations were disseminated beyond the academic realm.

\section{Purpose and Significance of the Study}

This study drew upon the expertise of people with ADRD to determine relevant strategies to improve ADRD clinical research participation rates. It contributes insights to the current knowledge base surrounding factors affecting clinical research recruitment and emphasizes motivational strategies among individuals with ADRD. Additionally, this dissertation is the first to use a civic engagement approach to study clinical research recruitment, as well as the first to apply the Culture of Health Action Framework within 
this specific field. Furthermore, this study obtained information from both academic and community stakeholders through $\mathrm{CAB}$ membership and interviews and supported calls by advocates and academics to include the people with ADRD as research partners (Tanner, 2012; LEAD Coalition, 2017; Kremer, 2017; NIA 2018; Gitlin et al., 2018; GilmoreBylovskyi et al., 2019). Lastly, it aligns with the NIA's national strategy to conduct more research on factors affecting ADRD clinical research participation and fills an important gap in the literature: factors and strategies derived from the expertise of those living with ADRD.

\section{Summary}

ADRD prevalence rates will continue to rise as the aging population grows. The efficacy of an intervention to prevent, treat, or cure ADRD can only be tested through clinical research, which will only be successful if individuals with ADRD are motivated to participate and strategies backed by evidence from people with ADRD are developed. Strategies have been identified by ADRD clinicians, researchers, and advocates, but not by the people with ADRD themselves. By identifying their needs, this research study served as the first stage in that process. In Chapter 2, a review of the literature that formed the basis for the design of this study is presented. 


\section{CHAPTER 2: REVIEW OF THE LITERATURE}

\section{Chapter Organization}

This chapter presents a review of the literature that provides the foundation for the study. The follow topics are discussed:

1. Introduction: the case for identifying facilitators and barriers to ADRD clinical research participation.

2. An overview of ADRD including its pathology, prevalence, current treatment, supports and services, and research directions.

3. A review of ethical guidelines that have shaped clinical research, including seminal guidelines, national NIH regulations, facilitators and barriers related to general clinical research participation, and factors related to ADRD research participation.

4. Discussion of the theories and frameworks that pertain to this study, including organizational theories within the school of open rational systems, policy process theories, civic engagement approaches and the Culture of Health Action Framework.

5. Literature synthesis and conclusion.

\section{Introduction}

In most developed nations, individuals no longer die from acute conditions such as infection and trauma; rather, they die from chronic conditions that last years or decades 
(World Health Organization [WHO], 2017). As more people survive to old age, and the national and global population grows to record numbers, the number of ADRD cases has drastically increased and will continue to do so over the next few decades. Globally, over 46 million people were diagnosed with dementia in 2015 , costing an estimated 816 billion dollars (Prince et al., 2016).

WHO declared ADRD a public health crisis in 2012 and released their response plan in 2017 (WHO, 2017). Among their priorities is the need for innovative research and a specific call for collaboration between academic institutions and engaged stakeholders, including people with ADRD, to find prevention strategies, treatment options, and/or a cure for people with ADRD (WHO, 2017). The NIA followed the next year, releasing their National Strategy for Increasing Participation in Alzheimer's Disease and Related Dementias Research in October 2018. Their four overarching goals are: 1) increase awareness and engagement; 2) build and improve research infrastructure; 3 ) engage local communities and support participants; and 4) develop an applied science of recruitment (NIA, 2018). As described in Chapter 1, two important actions should be taken to further improve this national strategy: continue research to identifying barriers, facilitators, and recommendations to improve research participation and develop strategies used that draw upon the expertise of people living with ADRD. This research study does both.

\section{Overview of ADRD}

This section summarizes the literature and current knowledge surrounding ADRD. The first third of this discussion focuses on the trajectory of the dementias within the 
ADRD family, including their history, epidemiology, diagnosis and treatment.

Current services and supports for individuals with ADRD are then discussed, focusing mostly on long-term supports and services (LTSS). This section concludes with an overview of current ADRD research, including the progress that has been made in some areas, the stagnancy in others, and current and future pathways that ADRD researchers are taking in their investigations. This review of the literature is necessary to provide a foundation for the context of this dissertation research. In order to study ADRD clinical research recruitment and retention, one must first consider why discovering ADRD prevention strategies and treatment options is a high global priority.

\section{ADRD subtypes}

Dementia symptoms have been written about since ancient times, and subtypes of dementia were distinguished in the late 1800s; however, ADRD was not commonly researched nor written about until the 1970s (Boller \& Forbes, 1998). With the exception

of familial Alzheimer's disease (AD) and vascular dementia, there is still no known cause of ADRD. More is known, however, about each dementia's unique pathology.

Alzheimer's disease: AD's pathology consists of two types of protein buildups in the brain: amyloid plaques and neurofibrillary tangles (Villemagne et al., 2013). It is not clear how these buildups affect the brain, but researchers do know that the tangles inhibit the protein from carrying out their typical tasks. Other pathological signs include brain inflammation, caused by immune cell attempts to remove the protein buildups, and brain atrophy due to cell loss (NIA, 2017). A rarer form of Alzheimer's disease, affecting less than one percent of the total population, is caused by genetic mutations of either amyloid 
precursor proteins or presenilin 1 and/or 2 proteins (Bekris, Yu, Bird, \& Tsang, 2010). Individuals with these genetic mutations will develop AD sometime between their 30s and 50s (Goldman, Hahn, \& Bord, 2011). There are currently 5.8 million people estimated to be living with AD in the United States and an additional 1 million living with related dementias, including frontotemporal, Lewy body, vascular, and mixed dementias( (Knopman \& Roberts, 2011; Plassman et al., 2007; Zweig \& Galvin, 2014; NIH2019a, 2018; Alzheimer's Association, 2019a;). However, there is speculation that this number is actually much higher, given the number of people who are misdiagnosed or do not receive a diagnosis (Boustani, Peterson, Hanson, Harris, \& Lohr, 2003; Bradford, Kunik, Schutlz, Williams \& Singh, 2009; Lang et al., 2017).

Dementia with Lewy bodies: The pathological diagnostic sign of dementia with Lewy bodies (DLB), Lewy bodies, are deposits comprised mainly of alpha-synuclein proteins that abnormally develop in brain cells (Gomperts, 2016). First discovered in Dr. Alois Alzheimer's lab by Frederick Lewy in 1912, DLB was classified as its own subtype of dementia in the 1990s and its clinical criteria were most recently updated in 2015 (Gomperts, 2016; McKeith et al., 2017). Like AD, it is difficult to assess the true number of people with DLB; due to its similar symptomology and pathology, many cases are misdiagnosed as AD or Parkinson's disease and are not properly diagnosed as DLB until autopsy (McKeith et al., 2017).

Frontotemporal dementia: Frontotemporal dementia (FTD) is another dementia commonly misdiagnosed as AD. The symptomology of one subset of FTD, Pick's disease, was first identified in 1892 by Arnold Pick (Snowden, Neary, \& Mann, 2002). 
However, it was assumed to be $\mathrm{AD}$, and it was not until more research comparing the two dementias was conducted in the 1980s that FTD was distinguished as its own subtype (Neary, Snowden, Northen, \& Goulding, 1988; Snowden et al., 2002). Pathologically, FTD is very similar to AD, except all protein build-ups occur strictly in the frontal and temporal lobes (Alzheimer's Association, 2018a). Clinically, FTD presents with behavioral and movement symptoms before cognitive symptoms. Approximately 60,000 people live with FTD in the US, most of whom are diagnosed between the ages of 45-64 (Galvin, Howard, Denny, Dickinson, \& Tatton, 2017). The age range is in part why the socioeconomic burden is greater for people with FTD than AD as they are often in their top wage point in their life and may be caring for young children at home (Galvin et al., 2017; The Association for Frontotemporal Degeneration, 2018). Furthermore, because of their age and behavioral and movement symptoms, people with FTD are often misdiagnosed with a behavioral or movement disorder (FTD Association, 2018).

Vascular dementia: Vascular dementia was first recognized as a dementia subtype in the late 1800s but was not assigned clinical criteria until the 1990s (Battistin \& Cagnin, 2010). It is the second most prevalent dementia in the United States and Europe and the most prevalent in Asian countries, accounting for nearly 50\% of all reported dementias in Japan and China (Battistin \& Cagnin, 2010). Vascular dementia is caused by restricted blood flow to the brain due to strokes or other vascular events, leading to damaged vessels and brain lesions that result in symptoms similar to AD (Love, 2005; Alzheimer's Association, 2018b). Slightly more than one in four people who have a stroke develop vascular dementia within one year (Kalaria, Alinyemi, \& Ihara, 2016). 
Mixed dementias: Mixed dementias do not have their own pathology; rather, they are a combination of two distinct subtypes of dementia. Generally, mixed dementia cases are comprised of pathology of $\mathrm{AD}$, the most common form of dementia, and vascular dementia, the second most common type of dementia (Schneider, Arvanitakis, Bang, \& Bennett, 2007).

\section{Diagnosis, risk factors and prevention, and treatment}

This section discusses factors affecting a clinical diagnosis of ADRD, risk factors and prevention of ADRD, and treatments for ADRD.

Diagnosis: Proper diagnosis of ADRD is crucial in order to treat symptoms early and obtain appropriate services and supports. However, a recent global meta-analysis of 23 studies found that 26,807 out of $43,446(61.7 \%)$ cases of dementia were originally undetected, with the prevalence in North America slightly higher than the global average at 27,328 (62.9\%) cases (Lang et al., 2017). The study also found that rates of undetected diagnosis were higher when the individual was male, younger than 70 , or had seen a general practitioner rather than a specialist (Lang et al., 2017). The underreporting of diagnoses may also be due to therapeutic nihilism, where clinicians fail to diagnose with patients with dementia because of their belief that nothing could be done (Aminzadeh, Molnar, Dalzier, \& Ayotte, 2012). Additionally, many people chose not to seek a diagnosis for their symptoms due to the stigma and fear of having a chronic, terminal illness (Chin, Negash, \& Hamilton, 2011; Grill \& Galvin, 2014; Millenaar et al., 2016; Hermann et al., 2018). 
Until recently, ADRD could not be confirmed until autopsy. PET scans are now being used outside of research studies to identify plaque buildup consistent with Alzheimer's disease and can give a confirmatory diagnosis of Alzheimer's disease (Rabinovici et al., 2019). This poses interesting problems as people become diagnosed before clinical symptoms manifest, but an investigation of this is outside the scope of this dissertation. Probable ADRD is typically diagnosed using neurocognitive assessments, such as the Mini Mental State Exam (MMSE) or the Montreal Cognitive Assessment (MoCA). The MMSE is best used to distinguish between healthy cognitive functioning versus impaired functioning, whereas the MoCA is used for distinguishing between mild impairment seen in MCI and early-stage AD (Trzepacz, Hochstetler, Wang, Walker, \& Saykin, 2015). Though Caucasian individuals are more likely to be diagnosed at earlier stages, African Americans are significantly more likely to develop ADRD (Danner et al., 2011; Williams et al., 2011; Chin et al., 2011; NIA, 2018).

Biologically, ADRD disparities could be due to higher prevalence of cardiovascular diseases and diabetes and/or genetics polymorphisms, abnormal changes in genetic material, that are unique to or more prevalent in individuals of African descent (Chin et al., 2011).Screening African Americans for ADRD can be more ambiguous. The MMSE has a high rate of false positives among African Americans (Stephenson, 2001; Chin et al., 2011). Additionally, providers who administer the MMSE may have racial bias (Stephenson, 2001; Wood, Giuliano, Bignell, \& Pritham, 2006; Chin et al., 2011). African Americans more often receive delayed and/or inadequate health services (Chin et al., 2011). African Americans are more likely to believe ADRD symptoms are a sign of 
normal aging and find dementia to be a more stigmatizing disease as compared to white adults (Espino \& Lewis, 1998; Roberts et al., 2003; Connell, Roberts, McLaughlin, \& Akinleye., 2009; Chin et al., 2011). Additionally, there is mistrust of the medical community by African Americans, further leading to delayed or lack of care (Katz et al., 2009; Chin et al., 2011).

Risk factors and prevention: The three biggest risk factors for developing ADRD are old age, family history, and the APOE gene (Lautenschlager, et al., 1996; Mahley \& Rall, Jr., 2000; Herbert, Weuve, Scherr, and Evans, 2013). These risk factors are not modifiable; however, they do not automatically result in ADRD. Scientists have identified several modifiable factors that will lower ADRD risk, including cardiovascular diseases (CVD), being physically active, a heart-healthy diet, having formal education, and being socially and mentally engaged. CVD, including stroke, hypertension, and high cholesterol, impact brain health because the brain uses much of the body's oxygen and energy, both carried through blood (Mergenthaler, Lindauer, Dienel, \& Meisel, 2013). Thus, scientists suggest engaging in heart-healthy activities to prevent CVD and reduce risk of developing ADRD. This includes being physically active, which increases blood flow to the brain, eating a heart-healthy diet that limits sugar and saturated fats, and not smoking (Blondell, Hammersley-Mather, \& Veerman, 2014; Morris et al., 2015; Hardman, Kennedy, MacPherson, Schoeley, \& Pipingas, 2016).

Having more formal education and working in intellectually stimulating occupations reduces risk (Sando et al., 2008; Pool et al., 2016). Additionally, being socially and mentally engaged also reduces risk, though the reasons why are unknown 
(Wang, Karp, Windblad \& Fratiglioni, 2002; Yates, Ziser, Spector, \& Orrell, 2016).

Lastly, associations between ADRD and head trauma have been found, especially in cases of younger-onset dementia (Plassman et al., 2000; Gardner \& Yaffe, 2014); the Alzheimer's Association suggests individuals protect their heads through seatbelt and helmet use and fall-proofing homes (Alzheimer's Association, 2019a).

Treatment: AD is the only form of ADRD with FDA-approved medications. The treatment does not provide relief of the disease itself, but simply offers symptom management. Medications to treat cognitive symptoms include galantamine, rivastigmine, donepezil and memantine (Yanniopoulou \& Papegeorgiou, 2013). The first three are primarily used for mild to moderate cases of $\mathrm{AD}$, and the last is used for moderate to severe cases (Yanniopoulou \& Papegeorgiou, 2013). Often, treatment delays the progression of cognitive symptoms for six to twelve months, though it is ineffective for some (Yanniopoulou \& Papegeorgiou, 2013; Alzheimer's Association 2018d). The second group of medications, which include antidepressants, anxiolytics, and antipsychotics, are for behavioral changes associated with AD (Yanniopoulou \& Papegeorgiou, 2013; Canevelli et al., 2016). These medications are often prescribed "offlabel," meaning that the medication has not been approved for this specific use (Canevelli et al., 2016). There is also controversy surrounding the practice of prescribing off-label medications for behavioral symptoms of ADRD as they are often prescribed before nonclinical interventions are attempted (Canevelli, et al., 2016).

There are no FDA-approved medications for related dementias. DLB is treated off-label with AD symptom medications, antidepressants, and anxiolytics (Alzheimer's 
Association, 2018e). Antipsychotics are not prescribed as many people with DLB have experienced life-threatening side effects (Lewy Body Dementia Association, 2015; Alzheimer's Association, 2018e). FTD is pharmacologically treated with antidepressants and antipsychotics; some clinicians also prescribe medications for AD and Parkinson's, but they are not frequently found to be effective (Penn Frontotemporal Degeneration Center, n.d.). Vascular dementia is treated with AD medications as well as hypertension, high cholesterol, and diabetes medications to lower risk of vascular dementia-causing cardiovascular events (Mayo Clinic, 2014).

\section{Current services and supports for ADRD}

Given the nature of ADRD, LTSS are utilized when the disease progresses. LTSS include in-home services (such as personal care and respite), adult day care, communitybased care (CBC) which includes residential care (RC), assisted living facilities (AL), and memory care facilities (MC), and nursing homes. In Oregon, Medicaid can be utilized for all LTSS, but is contingent on the providers' willingness to accept Medicaid. Those individuals who are higher-income can afford private-pay LTSS; middle-income individuals must spend down their assets on private pay LTSS until they become eligible for Medicaid. Oregon Project Independence, overseen by the Oregon Department of Human Services and jointly run by regional Area Agencies on Aging and service providers, offers in-home services, such as personal care and respite, for non-Medicaideligible adults, with no age restriction for those with an ADRD diagnosis, for a $\$ 5.00$ annual fee plus a monthly sliding scale fee. However, residential LTSS is generally utilized in advanced stages of ADRD. 
In Oregon, roughly $25 \%$ of $\mathrm{AL}$ residents, $50 \%$ of $\mathrm{RC}$ residents, and virtually all MC residents have dementia (Carder, Tunalilar, Elliot, \& Dys, 2017). The average monthly rates for Oregon's CBCs are $\$ 3,667$ for ALs, $\$ 3,770$ for RCs, and $\$ 5,410$ for MCs (Carder et al., 2017). Though 79\% of CBCs accept Medicaid, just $41 \%$ of residents pay with Medicaid (Carder et al., 2017). This low percentage rate is partially attributed to ineligibility, it could also be attributable to geographic disparity: CBCs are typically located in areas with higher levels of education and income (Stone, 2011; Carder et al., 2017). In Oregon, one in five nursing home residents has ADRD (Mendez-Luck, Luck, Larson, \& Dyer., 2017). More than one-fifth of Oregon counties, all of which are rural and low-income, do not have nursing homes (Mendez-Luck et al., 2017). People with ADRD who utilize residential LTSS may have to live far distances from their known environment.

Many people with ADRD solely rely on informal caregivers due to cultural or financial reasons. Compared to non-caregiving peers, caregivers are at risk for stress, insomnia, fatigue, depression, anxiety, stress-related illnesses, and their own diagnosis of dementia (Norton et al., 2010; /NAC/AARP, 2015). These risks are even greater for caregivers of people with ADRD, who perform more ADLs, IADLs, medical care, and key activities than their non-ADRD caregiving counterparts. The burdens of caregiving may affect their caregiving abilities, and those with high caregiver burden are more likely to place the person with $\mathrm{ADRD}$ in the first available $\mathrm{CBC}$ or nursing home rather than proactively searching for residences that best meet their needs and preferences (Ball et al. 2008; Stone, 2011; Adelman et al., 2014; NAC/AARP, 2015). The Oregon Family 
Caregiver Program, federally funded by the Older Americans Act and directed by Area Agencies on Aging, provides caregiver education and respite opportunities, as does Oregon Project Independence (Oregon Department of Human Services, 2015; Oregon Department of Human Services, 2017). The Alzheimer's Association also provides caregiver support programs and referrals to local services.

In a survey of African, Hispanic and Asian American ADRD family caregivers, many perceived caregiving as a personal responsibility that does not require seeking help or felt stigmatized seeking services both within their community and the healthcare system. In some cases, their partners with ADRD did not want to seek help, which their caregivers respected (Chin et al., 2011; Aranda, 2017). However, many did not seek help due to experiences of discrimination in the health system, language barriers, uncertainty about receiving help, or belief that nothing could be done (Chin et al., 2017; Aranda, 2017). This lack of help is significant given their higher prevalence of ADRD in minority communities: they are at higher risk of negative consequences of caregiving yet receive the least amount of support from the health and long-term-care systems.

At the 2017 ADRD Research Summit in Bethesda, Maryland, Aranda (2017) offered several strategies for improving dementia care for disadvantaged groups, including:

"a) investing in diversity-focused research on care, services, and supports; b) coordinating public awareness and outreach efforts with culturally-tailored brain health messaging and information on health, LTC, and community-based services; c) improving quality and access to care, services, and supports in multiple languages, for at-risk or underserved groups, geographical regions, etc.; d) growing the enrollment of underrepresented groups in clinical trials and services research through recruitment strategies and study designs that better reflect community and organizational realities; e) recruiting and training a more 
diverse provider and scientific workforce to address delays in diagnosis, improve treatment, and quality care, supports, and services; f) decreasing the gap in translation of research to sustained practice based on the latest findings in dissemination, implementation, and improvement sciences; and g) examining large-scale policies that promote person-, family-, and community-centered care across multiple systems, educational, employment-workplace; economic, health and LTC, environmental, immigration, etc.” (Aranda, 2017).

Employing these strategies may improve access, utilization, and trust of LTSS

among minority communities. Trust-building and strengthening the relationship between these communities and the LTSS sector may carry over to all health and research sectors; however, it would require similar efforts from those sectors.

\section{Research progress}

In the 1990s, efforts in ADRD research increased upon the realization that Baby Boomers would begin to age in the next decade. During this decade, investigators established three principles to guide their research: ADRD is heterogeneous, its onset can occur up to 40 years before "clinical manifestation," and interventions needs to target the pathophysiological, symptomatic, and social aspects of the disease (Khachaturian, 1998).

Two decades later, investigators have begun to distill these three principles into more focused areas. At the 2016 ADRD Summit in Maryland, research priorities included a) improving diagnostic skills; b) more effective dialogue, programs, and partnerships between the NIH and nongovernment organizations; c) address health disparities; d) initiate more clinical trials for DLB, particularly for diverse populations; e) create international research trial networks for FTD; and f) learn more about the association between vascular factors and dementia (Corriveau et al., 2017). 
ADRD clinical trials have the highest failure rate of any disease at $99.6 \%$, which is due to lack of efficacy as well as slow or failed participant recruitment (Cummings et al., 2014; Cummings et al., 2017). Clinical research investigators instead have shifted focus from treatment to diagnostics and prevention. Researchers have identified biomarkers, such as amyloid beta peptides measured in cerebral spinal fluid and blood, that could be used to diagnosis ADRD before clinical symptoms emerge (Ahmed et al., 2014; Nabers et al., 2018). One encouraging study presented at the 2017 Alzheimer's Association International Conference (AAIC) suggested one-third of dementia cases could be prevented if nine lifestyle factors (active hypertension treatment, more childhood education, exercise, maintenance of social engagement, management of hearing loss, reduction of tobacco use, and diabetes, depression, and obesity maintenance) were addressed (Livingston et al., 2017). AAIC 2018's plenary session highlighted a potential breakthrough: an amyloid anti-body being tested in a clinical trial has substantially reduced pathological and clinical symptoms of early AD (Swanson et al., 2018). The study has moved to Phase 3 to test the antibody's safety and efficacy (Clinicaltrials.gov, 2019). There were no data from any large clinical trials presented at AAIC in 2019. Instead, presenters highlighted findings from diagnostic and preventive studies, which centered around biomarkers, imaging, and technology (Bowman Rogers, 2019; Fagan, 2019; McCaffrey, 2019).

Some pharmaceutical companies have halted their ADRD research to focus on other efforts, prompting speculation that they dropped out to pursue more profitable endeavors (Axovant, 2017; DeStrooper, 2018; Hawkes, 2018; Lietzan, et al., 2018). 
Despite this reduction in funding and study opportunities, academic and government research centers, in addition to remaining pharmaceutical companies, such as Biogen and Eisai, are still funding and conducting clinical research and are actively recruiting participants (Pickett, 2018, DeStrooper, 2018; Alzheimer's Association, 2018b). Biogen renewed a previously halted trial and applied for FDA approval for a new treatment, aducanumab, after new analyses showed some improvement among participants who received the drug (Biogen, 2019).

In March 2018, the NIA began crowdsourcing public ideas for ADRD research participation with the goal of developing and implementing national strategies to facilitate recruitment (Masliah, 2018). As of December 2019, Congress had allocated 28 billion dollars to ADRD with the goal of finding a prevention or treatment by 2025 (Alzheimer's Association, 2019b).

\section{Conclusion}

The debilitating progressive nature of ADRD, the toll on informal caregivers (particularly among people of color) and the health system, and the lack of a prevention or cure reinforces the WHO and U.S. Congress's goal. In order to improve the odds of reaching the Congress's 2025 deadline, efforts to improve research participation are needed. The next section discusses this aspect further.

\section{Clinical Research}

Clinical research determines the safety and effectiveness of interventions, including medications, devices, and diagnostic products. Clinical trials, a subset of clinical 
research, prospectively assigns subjects to an intervention in order to evaluate its effects on human subjects (NIH, 2017b). Clinical trials consist of four phases, described below in Table 2.1.

\section{Table 2.1 Overview of Clinical Trial Phases}

\begin{tabular}{|c|l|}
\hline Phases & \multicolumn{1}{c|}{ Definition } \\
\hline Phase 1 & $\begin{array}{l}\text { The safety of an intervention is assessed over several months among 20-100 } \\
\text { healthy volunteers or people with the condition being studied. }\end{array}$ \\
\hline Phase 2 & $\begin{array}{l}\text { The intervention's efficacy is assessed across hundreds of individuals over } \\
\text { several months to two years. }\end{array}$ \\
\hline Phase 3 & $\begin{array}{l}\text { The intervention's effectiveness, benefits, and range of adverse reactions are } \\
\text { tested across hundreds to thousands of people over one to four years. }\end{array}$ \\
\hline Phase 4 & $\begin{array}{l}\text { The intervention has been approved by the FDA and can be purchased by the } \\
\text { general public, but studies are still conducted to compare it to other } \\
\text { interventions, its long-term effects on health and quality of life, and its cost- } \\
\text { effectiveness. }\end{array}$ \\
\hline
\end{tabular}

In order to successfully find a prevention or treatment by 2025, thousands of individuals with ADRD must be willing and able to participate in clinical research. Additionally, efforts to improve ADRD clinical research recruitment and retention must be balanced with good ethical and clinical regulatory adherence, organizational structures, and the community's needs. To articulate why the balance is needed, and how to achieve that balance, this section is divided into four parts. First, ethical guidelines for conducting human subject research are discussed. This is followed by a description of current national clinical research regulations. Next, barriers and facilitators to clinical research participation are identified and categorized as policy, system, organizational, and personal factors. Lastly, factors that are specific to ADRD research participation are discussed. 


\section{Ethical guidelines pertaining to clinical research}

Underlying the duty to protect all participants, and especially those "vulnerable to coercion or undue influence", is the concern for the protection of human research subjects (45 CFR 46, 2018, p. 9). This section describes clinical research guidelines that aim to protect the health and safety of participants. It begins with an overview of two early guidelines for safety and ethical standards in human subject research: the Nuremberg Code and the Declaration of Helsinki. The section then moves into a discussion of two unethical research studies that occurred despite these guidelines, and the subsequent policy actions amid public outrage.

The Nuremberg Code: The first of three standards protecting the rights of human subjects in biomedical research is The Nuremberg Code (The Nuremberg Code, 1947). Established to ensure that the horrific ordeal that individuals subjected to research during the Holocaust never occurs again, it stresses the importance of voluntary and informed consent:

"The voluntary consent of the human subject is absolutely essential. This means that the person involved should have the legal capacity to give; should be so situated as to be able to exercise free power of choice, without the intervention of any element of force, fraud, deceit, duress, over-reaching, or other ulterior form of constraint or coercion; and should have sufficient knowledge and comprehension of the elements of the subject matter involved as to enable him to make an understanding and enlightened decision." (The Nuremberg Code, 1947, p. 1).

Under these terms, the only individuals able to consent to participation in research are adults with the cognitive capacity to understand the purpose, risks, and benefits of a study. Additionally, a participant's decision to enroll must be an autonomous one, free of clinician, caregiver, or other proxy coercion. Under these guidelines, people with ADRD 
would not be able to participate in research due to potential diminished cognitive capacity and concerns of coercion.

The Declaration of Helsinki: The 1964 Declaration of Helsinki was the first position statement to promote the use of a healthcare proxy for a person unable to give informed consent, provided that the research conducted focuses specifically on the benefit to the person's disease or disability and is of minimal risk. The Declaration proclaims that "it is the mission of the doctor to safeguard the health of the people. [The doctor's] knowledge and conscience are dedicated to the fulfilment of this mission" (World Medical Association, 1964, p. 1). This statement sets the precedent that the individual enrolling in the study is a patient first and a participant second. The guidelines also stipulate that a person with ADRD could participate in research so long as there is a potential of a direct benefit (World Medical Association, 1964).

It is important to note that despite these ethical standards, unethical research has continued on occasion. In 1966, Henry K. Beecher, a physician and medical ethicist, published "Ethics and Clinical Research," which described 22 cases of unethical research. One notable example is a 1963 case of an immunologist injecting cancer cells into 22 Jewish Chronic Disease Hospital residents in Brooklyn, NY, many of whom had dementia and were Holocaust survivors, without voluntary informed consent (Beecher, 1966; Arras, 2008). The investigator, Chester Southam from the Sloan-Kettering Institute for Cancer Research, in 1963 wanted to determine if the human immune system could fight off injected cancer cells. Despite arguing that it was safe, he defended his reasoning 
for not injecting himself by stating, "Let's face it, there are relatively few skilled cancer researchers, and it seemed stupid to take even the littlest risk" (Langer, 1964, p. 49).

One notable example not included in Beecher's article was the Tuskegee Syphilis Study, which began in 1932. What was supposed to be a six-month study on Black men living with syphilis in rural Alabama became a 40-year study on the pathology of untreated syphilis. Researchers not only withheld curative antibiotics from study participants after they became available in the 1950s, but also conspired with local health centers and the military to ensure that participants did not receive penicillin (Jones, 1993). Despite ethical concerns raised by the medical community, it was not until a US Public Health Services whistleblower contacted an Associated Press reporter in 1972 that this study was publicized and subsequently ended after public outcry (White, 2000).

After numerous Congressional hearings on clinical research in the United States, the National Research Act was passed in 1974. The Act established the National Commission for the Protection of Human Subjects of Biomedical and Behavioral Research [hereafter referred to as The Commission], creating ethical guidelines for human subject research and institutional review boards (IRBs) such as The Belmont Report and 45 CFR 46 (The Commission, 1979; Rice, 2008).

The Belmont Report: The Commission gleaned their clinical research standards from three ethical principles: respect for persons, justice, and beneficence. They released their ethical guidelines, known as The Belmont Report, which safeguards the right to autonomous and informed decision making and stresses the importance of protecting those who are legally incapable of giving autonomous informed consent (The 
Commission, 1979). It also ensures that researchers will equally distribute the maximum benefit and least amount of risk and harm to every patient without exploiting any vulnerable people or groups. The Belmont Report notes the necessity for research that may benefit society despite not providing a direct benefit to the individual involved (The Commission, 1979). The Commission specifically references research with children, stating: "effective ways of treating childhood diseases and fostering healthy development are benefits that serve to justify research involving children -- even when individual research subjects are not direct beneficiaries" (The Commission, 1979, p. 5). Similarly, one could infer that it is ethical for people with ADRD to participate in clinical research for societal benefit, such as finding effective ways to treat ADRD, despite not receiving an individual benefit.

Each of the guidelines provided more flexibility than the one beforehand while still maintaining safeguards within human subject research, illustrated in Table 2.2. 
Table 2.2: Ethical Guidelines in Clinical Research

\begin{tabular}{|l|l|l|l|}
\hline Guideline & Year & Key Contribution & Relevance to ADRD Research \\
\hline $\begin{array}{l}\text { The Nuremberg } \\
\text { Code }\end{array}$ & 1947 & $\begin{array}{l}\text { Subjects must } \\
\text { autonomously and } \\
\text { voluntarily provide } \\
\text { informed consent. }\end{array}$ & $\begin{array}{l}\text { People with ADRD are unable to } \\
\text { participate in clinical research. }\end{array}$ \\
\hline $\begin{array}{l}\text { Declaration of } \\
\text { Helsinki }\end{array}$ & 1963 & $\begin{array}{l}\text { Subjects can enroll via } \\
\text { proxy consent so long } \\
\text { as the research is } \\
\text { directly beneficial and } \\
\text { is of minimal risk. }\end{array}$ & $\begin{array}{l}\text { People with ADRD can participate } \\
\text { in directly beneficial, minimal risk } \\
\text { studies if they have a study } \\
\text { partner. }\end{array}$ \\
\hline $\begin{array}{l}\text { The Belmont } \\
\text { Report }\end{array}$ & 1979 & $\begin{array}{l}\text { Subject can enroll via } \\
\text { proxy consent so long } \\
\text { as the research benefits } \\
\text { society. }\end{array}$ & $\begin{array}{l}\text { People with ADRD can participate } \\
\text { in minimal risk studies if they } \\
\text { have a study partner and the study } \\
\text { would benefit those with ADRD. }\end{array}$ \\
\hline
\end{tabular}

The Nuremberg Code safeguarded against exploitative experiments and fraudulent acts disguised as medical research, but it also blocked those who could not give informed consent from receiving beneficial treatment that they could only get through research participation. The Declaration of Helsinki remedied that by allowing proxy permission for those who are unable to give consent; however, it forbids enrollment by proxy into research unless the research was directly therapeutic and of minimal risk, though neither term is defined (World Medical Association, 1964). The Belmont Report amends this by allowing proxy enrollment in studies that may not provide a direct benefit but will be beneficial to the population affected by the same ailment as a whole and is of low risk (The Commission, 1979).

\section{National clinical research regulations}

Since the 1970s, the US has actively regulated clinical research, primarily to ensure good ethical and clinical quality. The Office of Human Research Protections 
(OHRP) "provides clarification and guidance, develops educational programs and materials, maintains regulatory oversight, and provides advice on ethical and regulatory issues in biomedical and behavioral research" (OHRP, n.d., p. 1). 45 CFR 46, a regulation promulgated by the Department of Health \& Human Services (HHS) fulfills OHRP's responsibilities by ensuring the safety of individuals participating in human subject research. However, these rules do not provide guidance for studies that involve participants with ADRD, stating: "the HHS regulations are silent on the consent procedures specific to subjects with impaired decision-making capacity, for example, as a result of trauma, mental retardation, some forms of mental illness, or dementia, whether temporary, progressive, or permanent" (OHRP, 2017, p. 10). Investigators and research funders have the ability to create their own procedures, provided they align with good ethical and safety standards, and can differ from clinic to clinic. One result of this means that a person with ADRD may not be eligible for a study at their local clinic but could be eligible if they lived elsewhere (Friedman et al., 2015; Mahon et al., 2016). The varying eligibility criteria contributes to the low participation rates in ADRD clinical research, making it more challenging to discover a possible prevention or treatment by 2025 .

Several policies have been implemented in recent years to encourage a larger scope of studies, participants, and collaboration. Federal rules established as directed by the NIH Inclusion Act of 1993 require NIH-funded research studies to: ensure inclusion of women and minority subpopulations; incorporate valid analyses of subpopulation groups in Phase 3 trials; not exclude participation based on cost; and initiate recruitment programs and outreach efforts (NIH, 1994). Subsequent updates in 2000 and 2017 
mandate that inclusion should be discussed in the research design plan, allow for exclusion if there is a clear and compelling reason for it, and require that valid analyses are submitted to ClinicalTrials.gov (NIH 2000; NIH, 2017c). Unfortunately, this only applies to NIH-funded studies and does not apply to the majority of ADRD clinical trials, as over 65\% are funded by biopharmaceutical companies (Cummings et al., 2017).

The NIH modified its definition of a clinical trial in 2014 to "a research study in which one or more human subjects are prospectively assigned to one or more interventions (which may include placebo or other control) to evaluate the effects of those interventions on health-related biomedical or behavioral outcomes" (NIH, 2014, p. 1). This expands the definition to include any study that involves human participants and tests a health-related intervention provided that participants are prospectively assigned to the intervention. The $21^{\text {st }}$ Century Cures Act, which stresses transparency of clinical research processes and outcomes as well as access, privacy, and confidentiality for participants, was signed into law in December 2016 (P.L. 114-255, 2016). This policy has led to the creation and modification of several additional policies.

In June 2016, the NIH passed a final rule that allows for a single IRB review of $\mathrm{NIH}$-funded multi-site studies and requires investigators and research staff of NIHfunded trials to complete Good Clinical Practice (GCP) training to "assure the safety, integrity, and quality of clinical trials" (NIH, 2016b, p. 1). In January 2017, the NIH enacted a policy addressing the transparency and dissemination of clinical trial results. Under this policy, investigators (apart from Phase 1 studies and studies without FDA regulated products) must register their trial on ClinicalTrials.gov within 21 days of the 
first participant enrollment and publish their findings within one year of trial completion (NIH, 2016c). Previously, investigators were only required to post their publications on NIH-funded studies to PubMed Central.

In September 2017, the NIH changed its confidentiality policy. Rather than requiring investigators to apply for certificates of confidentiality (COCs) to protect their participants' sensitive information, COCs are automatically distributed by the HHS Secretary for studies receiving federal funding (NIH, 2017d). Under this rule, disclosure of identifiable, sensitive information may only be made if: required by other federal, state, or local laws (e.g. communicable disease reporting); necessary for the medical treatment of the individual; the individual consents; or, for other scientific research that adheres to federal human subject regulations (NIH, 2017d).

In November 2017, the NIH announced its partnership with Open Researcher and Contributor Identification (ORCID), a nonprofit organization where researchers sign up to network and share information, reporting, and analyses. According to the NIH's Deputy Director for Extramural Research, the goal of this collaboration is to "make it easier for the scientific community to create measures and incentives for better scientific researchers such as openness, rigor, and impact” (Lauer, 2017, p. 1).

Most relevant to ADRD clinical research, NIH now prohibits investigators from excluding potential participants due to age without good justification (NIH, 2017a). This Inclusion Across the Lifespan Policy specifically cited studies that focus on cancer research as reasons for why this rule was necessary: for example, over one third of cancer patients are over the age of 75 , yet less than one in ten clinical research participants are 
over age 75 (Hurria et al., 2014). This also positively impacts people with ADRD, particularly those above 85 and younger than 65 , because studies must now include individuals whose ages are most affected by these diseases. Despite over one-third of people with ADRD being over age 85, just 8\% of ADRD clinical research participants between 2000 and 2015 were 85 or older (Banzi, Camaioni, Tettamanti, Bertele, \& Lucca, 2016). Additionally, ADRD clinical research frequently limits eligibility to those 65 and older (Clinicaltrials.gov, 2018). This is particularly important to those with earlyonset familial $\mathrm{AD}$, who are more motivated to participate in clinical research yet more likely to be excluded by age (Schneider, 2010; Strobel, n.d.). Additionally, this only applies to NIH-funded studies and is not applicable to the majority of ADRD trials.

\section{Barriers, Facilitators, and Strategies Influencing Clinical Research Participation}

In order to identify ADRD-specific barriers, facilitators, and strategies influencing clinical research participation, one must first identify factors affecting general clinical research participation. The discussion of barriers, facilitators, and strategies is divided into four subsections: policy, system, organizational, and personal. This is followed in each case with a subsection on ADRD-specific factors and a summarizing conclusion.

\section{Policy}

Governmental and institutional policies are intended to ensure the safety of participants and the quality of the study. However, these policies affect capacity to participate in clinical research. For instance, integrity of data collection and monitoring of research, while necessary to ensure ethical and clinical regulatory compliance, have 
detrimental effects on study expenditures and reduce funding allocated to participant recruitment and retention without evidence of the intended safety and quality benefits (Califf, 2006; Kramer, Smith, \& Califf, 2012; Hemminki, 2016). As compared to the UK, Canada, and Finland where IRBs are run by the government nationally or by local municipalities, many U.S. research institutions have their own IRBs to determine which research studies take place and how (Hemminki, 2016). Though rigid national laws do not permit flexibility and case-by-case decision making, Hemminki (2016) is concerned by high costs in USA and the lack of ethical considerations of research purposes and dissemination of results. She argues that the time spent auditing and checking procedural formalities does not make research more ethical, and, in fact, the rules, procedures, and cost may deter clinicians from conducting research (Hemminki, 2016). This aligns with Kramer and his colleagues' (2012) review of clinical research impediments in the United States.

Though the scope of the IRB has expanded over the last three decades as pharmaceutical companies began funding more studies, there is no evidence that the safety and quality of clinical research has improved with the additional oversight (Dilts \& Sandler, 2006; Kramer et al., 2012). For example, Dilts \& Sandler (2006) cited one single-site oncology study that required 29 checks by 11 different people in order to be IRB approved, yet the IRB and investigators were unable to verify if the safety or quality of the research had been improved by those extra checks. The NIH has taken steps to reduce bureaucratic practices, such as permitting a single IRB review and approval for a multi-site clinical research, but the directors acknowledge that more work still needs to be 
done to further enhance and advance clinical research (Hudson, Lauer, \& Collins, 2016; NIH 2017d).

Despite U.S. requirements to disseminate results of clinical trials via PubMed Central and ClinicalTrials.gov, there are still issues of transparency and respect of participants. Investigators of NIH-sponsored studies do not need to disseminate results outside of the aforementioned, nor are they required to use clear and concise language. Thus, results are formatted as they would be for a peer-reviewed publication, not for the average participant, and are rarely disseminated to the participant directly.

ClinicalTrials.gov is difficult to browse, though NIH directors plan to improve its formatting, featurability, and findability (Hudson et al., 2016). This directly contradicts what participants want; researchers have found that dissemination of results is so important to participants that, if they did not receive study updates, they would not enroll in a study again (Sood et al., 2009; Law et al., 2014). Disseminating results directly to those involved in research is frequently recommended in the literature (Israel et al., 2008; Carlisle et al., 2015; Mahon et al., 2016).

\section{System}

System-level factors include the interactions among research organizations, health and social organizations and the community. A significant aspect of their interactions involves the community within and between sectors. When communities are not active participants in these interactions, this may result in overlooked opportunities and barriers to research enrollment. For example, community members may not be aware that research studies exist, resulting in missed recruitment opportunities (Heller et al., 2014; 
Watson, Ryan, Silverberg, Cahan, \& Bernard, 2014; Mahon et al., 2016; Anderson et al., 2018). Lack of awareness of clinical research opportunities plays a large role in the diminished participation rates, though a large number of surveyed individuals say that they would participate if they knew of research opportunities (English et al., 2010; Grill \& Galvin, 2014; Anderson et al., 2018). There is a disconnect between where research organizations assume community members will hear about studies, and where community members assume they will hear about research studies. Most assume their providers will inform them, but many clinicians do not involve themselves with clinical research but are more likely to learn of them through media advertisements (Friedman et al., 2015; Anderson et al., 2018). Additionally, though research studies are often posted on clinical research websites, many individuals report being unaware of, or not knowing how to find, clinical research opportunities on the internet (Sood et al., 2009; English et al., 2010; Kramer et al., 2012).

Some clinicians may not view research as a necessary piece of their clinical practice; others are concerned about increasing their risk of being sued or utilizing limited resources such as time, money, and workforce (Sugarman, Getz, Speckman, Byrne, \& Emanuel, 2005; European Science Foundation, 2011; Kramer et al., 2012). In many schools, medical students are not properly trained in clinical research methods nor aware of clinical research opportunities at their academic medical center (Rahman et al., 2011, Kramer et al., 2012, Meador, 2015). Consequently, there are fewer physicians acquainting themselves with current clinical research, resulting in fewer opportunities to inform patients of research. 
Research organizations that are housed within, or partner with, healthcare providers may have access to patient data from an electronic health record (EHR) system. However, nonstandard nomenclature or not entering medical data in the same format, term, or entry area of the EHR may lead to gaps and overlook of potential participants (Califf, 2006; Richessen \& Krischner, 2007; Kramer et al., 2012). Mahon and colleagues suggested adding a checkbox in the EHR to allow providers, if given permission by their patients, to permit researchers to access their records and contact them about clinical research opportunities (Mahon et al., 2016). A more passive option is to post flyers or brochures around a clinic to inform clinic patients of local research opportunities (Mahon et al., 2016).

The NIA's (2018) National Strategy to Increase Alzheimer's Disease and Related Dementias Clinical Research Participation aims to recruit more people of color into research. In order for this to be successful, institutionally racist policies and practices needs to be recognized and mitigated. Structural or institutional racism, defined as "differential access to the goods, services, and opportunities of society by race," is a significant systemic barrier to the inclusion of people with color (Jones, 2000, p. 1212; Freeman et al., 2017).

Structural racism is well-known in the criminal justice and housing sectors, but also appears in the health and clinical research sectors (Delgado \& Stefanic, 2001; Freeman et al., 2017). One example is the need for a diagnosis of ADRD, a frequent eligibility criterion for ADRD studies. Though African Americans have a higher prevalence of ADRD in African Americans, they are more often diagnosed in the later 
stages of diseases, due to lack of access, transportation, language barriers, a lack of cultural competency, cultural beliefs about dementia and medicine, and real and perceived discrimination (Chin et al., 2011; Bonds \& Lyons, 2017). This delayed diagnosed results in a missed opportunity to participate due to low cognition scores (Chin et al., 2011; Grill \& Galvin, 2014; NIA, 2018). Other examples include requiring people to visit the clinic (which can exclude people of color who live too far away as a result of redlining and gentrification), failure to engage with African Americans because of assumptions that will not participate, and excluding cultural practices, such as collaborate decision making with friends and family members (Huang \& Coker, 2010; Chin et al., 2011; Danner et al., 2011; Penberthy et al., 2012; Frew Archibald, Hixson, \& del Rio, 2011; Grill \& Galvin, 2014; Johnson et al., 2015; Mahon et al., 2016; Freeman et al., 2017).

One mechanism to recognize and reduce these barriers is to analyze policies and practices using Critical Race Theory (CRT). CRT recognizes that a racist person does not need be present in order for racism to exist and persist within a system; many policies and practices are embedded in white privilege and supremacy (Delgado \& Stefancic, 2001). Additionally, CRT invokes the notion that identity and oppression is multi-dimensional, and intersectionality of gender, class, and nationality, as well as others, affect a person's disempowerment in various settings (Delgado \& Stefancic, 2001). CRT requires selfreflection and community engagement with marginalized populations for praxis to be realized (Ford \& Airhihenbuwa, 2010). 
Community engagement among research, health, and social sectors can facilitate clinical research recruitment and retention (Frew et al., 2011; Grill \& Galvin 2014; Watson et al., 2014; Mahon et al., 2016; Inungu et al., 2017; Gilmore-Bykovskyi et al., 2019). Community-engaged strategies result in the research organization's familiarization of target populations' risk behaviors, which are essential to inform effective prevention strategies as they can both affect and inform research design, recruitment, and retention (Voytek et al., 2011; Luzi et al., 2011; Inungu et al., 2017).

When research organizations partner with local health providers and social groups, they promote trust between the community and themselves (Gifford et al., 2002; Frew et al., 2011; Mahon et al., 2016; Inungu et al., 2017). For example, when neighborhood-based HIV medical and treatment programs and HIV support groups and services encouraged participation in an HIV-vaccine research, the likelihood of participation among members of a HIV-prevalent community increased (Frew et al., 2011). Recognizing and reducing other structural barriers, such as transportation and geographic distance, also facilitates community member buy-in and subsequent participation, (Giffords 2002; Frew et al., 2011; Mahon et al., 2016, Inungu et al., 2017; Croff et al., in press). Among marginalized communities, the CRT practices of selfreflection and community engagement is found to enhance trust and likelihood of research participation (Huang \& Coker, 2010, Frew et al., 2011; Grill \& Galvin, 2014; Johnson, Joosten, Wilkens \& Shibai, 2015; Inungu et al., 2017; Gilmore-Bylovskyi et al., 2019). 


\section{Organization}

Organizations and study sponsors frequently implement their own policies in order to adhere to good clinical and ethical standards. However, they may inadvertently cause barriers to clinical research recruitment and retention. For example, many research organizations' IRBs take several weeks or months to review applications, grants, and contract reviews, despite no proof that this extra time improves the quality of their studies, delaying research for long periods of time (Dilts \& Sanders, 2006; Kramer et al., 2012). Additionally, study funders, such as pharmaceutical companies, can impose additional risk-averse practices including $100 \%$ source-document verification and on-site monitoring without quality improvement evidence (Kramer et al., 2012). To combat these high cost practices, research organizations could provide non-local study sponsors with data that are protected and accessible and discuss through video conference.

Additionally, research institutions that are also academic or health centers do not always emphasize clinical research as a necessary skill. Students and new faculty may not be trained in clinical research or interpretation of randomized clinical trial results, and clinical research may be disincentivized in favor of basic research or general practice (Nathan \& Wilson, 2003; Windish, Huot, \& Green, 2007; Kramer et al., 2012). Studies conducted in the U.S. and the E.U. found that study eligibility criteria can severely restrict the enrollee pool of a research, making it difficult to obtain an adequate number of participants within the proposed time frame (Kramer, 2012; Adams et al. 2015; Mahon et al., 2016). Though the protocols aim to protect human subject safety, there are cases of private study sponsors requiring unsubstantiated ineligibility criteria, such as 
comorbidities or medications, to avoid risk or delay of FDA approval (Kramer et al., 2012).

Communication facilitates clinical research decision-making ways that both increase and decrease likelihood of enrollment (Burke, 2014; Jenkins \& Fallowfield, 2015). An organization's recruitment strategy may serve as a barrier to research participation. Monson and colleagues (2012) found that individually identifying and inviting people to participate in a study and offering a lengthy pamphlet on the study's information does not provide potential participants with enough comprehensible evidence to make a fully informed decision to participate or not. When utilizing group presentations to discuss clinical research, $92 \%$ of attendees later enrolled in the study (Monson et al., 2012, 288). This recruitment strategy has been especially effective with minority populations (Williams et al., 2011; Heller et al., 2014).

Actions that research organizations take to exemplify their gratitude or respect of the participant also affects the decision to participate in clinical research. For instance, $90 \%$ of subjects in a study on research participation values stated they wanted to be informed of study results after the research had been completed, and over two-thirds found this to be such an important indicator of the research institution's respect for them that they stated they would never participate in clinical research studies again if they were not informed (Sood et al, 2009, 244). These findings are consistent with other studies (Law et al., 2014; Anderson et al., 2018). African Americans' willingness to participate in research is facilitated by community trust building, outreach, and community partnership creation (Williams et al., 2011; Lang et al., 2013; Johnson et al., 2015). 
The discrepancy between what clinical research staff perceive they are doing versus what they actually do varies greatly. For instance, a large majority of staff recruiting HIV+ participants believed they actively recruited Black, Hispanic, and female individuals, yet felt most effective when interacting with white men (King et al., 2007). Staff also admitted to avoiding non-English speakers and active intravenous drug-users and did not directly market to racial and ethnic minority group members; staff reported they did so because they received penalties for loss-to-follow up and these population groups were more likely to drop out (King et al., 2007). This is consistent with other studies that failed to actively recruit African Americans (Uybico, Pavel, \& Gross, 2007; Huang \& Coker, 2010). This highlights an example of personally mediated racism, defined by Jones (2000) as differential assumptions or actions against others based on their race, whether intentional or not. The authors speculated that missed appointments might be due to lack of transport, ability to take off work, or child care, while avoidance of non-English speakers may be due to lack of translated materials and bilingual staff (King et al., 2007). King and colleagues (2007) suggested improving the diversity of providers and staff, using translated materials, and increasing community-based research as ways to improve trust between research teams and targeted participant groups.

Despite higher rates of chronic disease diagnosis and mortality rates and inclusive regulatory mandates, less than $10 \%$ of African Americans participate in clinical research (Owens, Jackson, Thomas, Friedman, \& Hebert, 2013; Perez-Stable, 2018). When Owens and colleagues (2013) interviewed African American adults about their perceptions for cancer clinical research, fear or mistrust was the most commonly cited reason for why 
they would not enroll. African American men and women more often referred to clinical research as "experiments," and African American men were more likely to refer to participants as "guinea pigs" (Owens et al., 2013). When Corbie-Smith and her team (1999) interviewed African Americans in a Southern state, the interviewees overwhelmingly viewed research as beneficial to themselves and society but believed research institutions do not have their best interests in mind. One example cited by respondents was the use of informed consent forms; they believed that the forms were not actually for the participant's safety but rather to protect the investigators from legal action (Corbie-Smith et al., 1999). Due to their experiences with institutional racism, as well as community effects from the Tuskegee Syphilis Study, the respondents expect “dishonesty" and "conspiracy" from research organizations (Corbie-Smith et al., 1999).

Dialogue and education between research organizations and the African American community can mitigate some of the distrust (Danner et al., 2011; Owens et al., 2013). However, it does not automatically increase participation; after a 4-week clinical research education workshop with African Americans, there was no significant change in likelihood of participating in clinical research, but they were more likely to cite concerns of time commitments rather than fear or mistrust (Owens et al., 2013).

Researchers' perceptions of African Americans also affect participation rates. African Americans are more likely to be deemed ineligible according to study criteria as compared to their white counterparts (Schneider et al., 1997; Chin et al., 2011; Penberthy et al., 2012). When investigators from Virginia Commonwealth University analyzed staff-reported cancer clinical trial ineligibility and refusal reasons, they discovered that 
African American and White cancer patients were similarly excluded due to comorbidities and cancer-related characteristics, but African Americans were significantly more likely to be excluded from the study due to perceived/documented incapacity to provide informed consent and expected noncompliance (Penberthy et al, 2012).

\section{Personal}

Though studies have found that many individuals would hypothetically participate in clinical research, willingness does not correlate with actual enrollment (Buchbinder et al., 2004; Grill \& Galvin, 2014; Inungu et al., 2017). Many individuals cite time commitments and scheduling issues, distance, and need for more information as reasons why they could not enroll (Penberthy et al., 2012; Owens et al., 2013; Gollhofer et al., 2015; Friedman et al., 2015). Others cite ambiguity of informed consent, fear of side effects, and fear of randomization (e.g. receiving a placebo) for why they would not consider participation (Penberthy et al., 2012; Owens et al., 2013; Friedman et al., 2015). Financial incentives, ease of transportation, receiving more information about studies, and partnering with trusted community leaders are frequently cited ways to improve the likelihood of participating (Brown et al., 2013; Golhoffer et al., 2015; Friedman et al., 2015, Mahon et al., 2016; Anderson et al., 2018; Croff et al., in press).

In addition to mistrust of research organizations, African Americans faced significant barriers to participating, wanting to make decisions collaboratively with family and recommendations against participating from family members and friends as reasons for not enrolling (Penberthy et al., 2012; Brown et al., 2013). Those who express 
willingness to participate, and those who have participated, cite altruism, benefit to themselves or someone they know, and financial incentives (such as free healthcare or stipends) as reasons they want to participate (Owens et al., 2013; Friedman et al., 2015; Gollhofer et al., 2015).

This evidence illuminates that though an individual has the final say in participating in clinical research, their decision is heavily influenced by a variety of factors. Decisions made in policy, system, and organizational spheres directly or indirectly affect the capacity for a person to enroll in a research study, and also their motivation to do so.

\section{ADRD-specific facilitators and barriers to participation}

Individuals with ADRD face additional barriers to research participation. Approximately 10-27\% of people with ADRD are eligible to participate in research due to comorbidities, incompatible medications, low cognition scores, and frailty (Grill \& Galvin, 2014). Additionally, study partners are required to participate with the individual with ADRD to ensure protocol adherence and appointment attendance, further complicating the process due to their own reasons for not being able to participate (Karlawish et al., 2008; Watson et al., 2014; Grill \& Galvin, 2014; Cary et al., 2015; Black et al., 2018). Though these barriers may differ from those of general clinical research, drawing upon the literature from other populations with similar barriers could assist with identifying further facilitators.

Adult children are more likely to serve as caregivers whereas spouses are more likely to serve as study partners (Karlawish et al., 2008; Cary et al., 2015). There is a 
general consensus among individuals with ADRD and study partners that proxy consent is appropriate, and most patients cede decision-making to their study partner (Karlawish et al., 2008). However, study partners make proxy decisions based on reasons aside from previously expressed preferences, including direct benefit to the person with ADRD, altruism, and caregiver support and education (Karlawish et al., 2008; Cary et al., 2015; Black et al., 2018). Study partners are more likely to agree to participation if they perceive the research as safe (Cary et al., 2015). Caregiver burden is not associated with refusal; in fact, many study partners perceive their role as an extension of caregiving responsibilities (Black et al., 2018). Common study partner barriers and facilitators to participation are similar to the general clinical research barriers (e.g. mistrust, direct benefit, travel), but also include unique reasons. For example, a barrier may be the emotional distress of not knowing answers or the realization of ADRD progression, whereas a facilitator may include caregiver support or education (Black et al., 2018). Individuals with intellectual disability (ID) are another community that have very low research participation rates, and often require a study partner (Lennox et al., 2005; Feldman, Bosett, Collet, \& Burnham-Roisa, 2013). When Lennox and colleagues (2005) conducted a study with individuals with ID, they found that study partners, both family members and care staff members, were not enrolling in the studies due to burden of care, burnout, and mistrust of the research staff. The team consulted with a community advisory board, comprised of adults with ID, parents of children with ID, support workers, an occupational therapist, and a professional advocate, to develop strategies for gaining community support and increasing recruitment. The team collaborated with an 
"insider" within an organization that serves adults with ID to call prospective participants to provide information about the study. Additionally, public information sessions were held to provide more details, answer questions, and establish a relationship with ID community members. Using these two strategies, recruitment and participation significantly improved.

Reasons for not participating within the ID community were similar to the experiences of people with ADRD. Family caregivers of people with ID commented that they did not have much financial or personnel support, and any additional time constraint, such as participation, was not a priority (Lennox et al., 2005). People with ID, their informal caregivers, and care workers also reported perceptions of discrimination by specialists and hospitals and may perceive research staff as an extension of that workforce (Lennox et al., 2005). Additionally, nearly $90 \%$ of the adults with ID eligible for the study required study partners due to their lower IQ scores, leading to the potential for refusal due to study partner dissent (Lennox et al., 2005; Feldman et al., 2014). Though ethically necessary to protect people with low cognition, requiring study partners also denies individuals who may have wanted to participate but are banned from inclusion without one. Lennox and his team (2005) recommended the use of participatory research strategies to allow the person with ID to be more of a partner than a participant.

To further promote inclusion of ID community members in research, academics, advocates and individuals with ID suggest modifications and accommodations, including: extra time, one-on-one support, taking breaks, simplifying the language in the informed consent form and other materials, and using figures and illustrations to improve 
comprehension (Lennox et al., 2005; Feldman et al., 2014; Nicolaidis et al., 2015; McDonald et al., 2016).

A provider's lack of time, cognitive assessment training, and knowledge of research opportunities to accurately refer eligible patients to ADRD research hinders likelihood of participation (Grill \& Galvin, 2014; Watson et al., 2014). As discussed in a previous section, not having a proper diagnosis in the first place inhibits the ability to participate in a clinical research. Churches and other religious institutions often serve as community centers for those with religious ties and may serve as another trusted source in addition to a provider. In a survey of clergy leaders about their church's dementiafriendliness, most churches reported dementia-inclusive events and practices such as shared meals, games, and frequent event reminders. However, the leaders also noted that they did not know where to find dementia resources or where to refer patrons to (Plunkett \& Chen, 2016). The investigators suggested networking with churches to develop programs that better serve patron needs (Plunkett \& Chen, 2016). A partnership between a research organization and a religious institution may establish trust between the two and facilitate recruitment.

In St. Louis, one Alzheimer's Disease Research Center (ADRC) has utilized many previously discussed motivators and strategies to improve research participation among African Americans with ADRD. The African American Outreach Satellite program (Satellite) works with the St. Louis Alzheimer's Disease Research Center to provide home-based assessments, dementia diagnoses and services, and recruitment opportunities for older African American community members to research studies. Over 
their $25+$ year history, they have developed sustainable relationships with older African American adults, caregivers, and community leaders as well as health care providers and local Area Agencies on Aging (Williams et al., 2011). Satellite's work with an African American advisory board, the Alzheimer's Association, local clergy and religious groups, and other community partners helped establish and implement a variety of outreach strategies to increase African American recruitment in ADRD clinical research (Williams et al., 2011). Over a ten-year period, African American participation rates increased from $8.6 \%$ to $19 \%$, comparable to the St. Louis population rates (Williams et al., 2011).

\section{Conclusion}

Scientists, the health and LTSS workforce, and policymakers expect an exponential increase in ADRD diagnoses in upcoming years, and hope for a prevention or treatment within the next seven (Collins et al., 2018; Alzheimer's Association, 2018a; Gates, 2017). Despite significant funding and calls for research participation, ADRD clinical research enrollment remains low due to a variety of policy, system, organizational, and personal factors. Ethical guidelines and clinical policies have been put in place to ensure that participants are allowed to participate in studies for individual and collective good but are protected from harm. However, for people with ADRD, this has led to significant restriction of opportunities. The literature review highlighted the barriers of requiring a study partner, but also offered strategies to improve participation and the use of modifications that can be utilized and promote inclusiveness for individuals with cognitive impairments. 
Many of the system and organizational level barriers center on communication, or lack thereof, between and among different communities, organizations, and sectors. Furthermore, structural racism has led to health and research participation disparities among African Americans. Identifying these barriers is the first step, addressing them is the second. Using the CRT approach, removing these barriers requires self-reflection and community engagement with mitigate power structures and emancipate African Americans from structural racism. It is a long, arduous process, but is imperative to ensure that the community with the highest prevalence of ADRD has the same opportunities to participate and is not impeded by former and current actions from medical and research organizations. As exemplified by the Satellite program in St. Louis, when efforts are made to listen to and include all members from various directions, the ability to significantly increase recruitment and retention of individuals with ADRD into clinical research is possible. The next section elucidates some of the theories and frameworks that support those efforts.

\section{Theories and Frameworks That Pertain to This Study}

This section of the literature review provides an overview of the organizational theories and policy frameworks that are relevant to this study. The first section begins with a discussion of relevant organizational theories. These theories, transaction cost and contingency theory, fall into the school of open rational systems. The next section describes the social construction framework and policy feedback theory, two relevant policy theories. The third section articulates the concept of civic engagement and 
connects it to the concept of research participation. Finally, the last section identifies and describes a framework that incorporates elements of civic engagement and the organizational and policy theories: the Robert Wood Johnson Foundation's Culture of Health Action Framework.

\section{Organizational theories}

Clinical research organizations are currently structured to adhere to both national and international good ethical and clinical regulations and practices, but also to meet the needs of the investigators and research staff in order to achieve the goal of finding a prevention strategy or treatment of ADRD by 2025. These regulations and practices, while important, are not flexible towards environmental needs such as the preferences or needs of people with ADRD. Additionally, some health and social sectors factors that further impede or facilitate an individual with ADRD's likelihood and ability to participate in research, such as time for a provider to discuss research opportunities, are outside of the research organization's control. Based on the body of literature reviewed, ADRD research organizations would benefit from a design that maintains the rigidity needed to meet their goals but also provides flexibility based on environmental needs and influences. Organizational theories that best describe the shift that research organizations should strive for are those within the school of open rational systems theory (Scott, 1987). Theories in this school assert that organizations are a means to a predetermined end but recognize the influence that environments can have on staff roles and decisionmaking structures. 
Transaction cost theory: Pioneered by Williamson (1975), transaction cost theory suggests that every transaction between two parties (both within and among organizations or groups) is subject to a cost. The theory has four components that are heavily based on market principles. Uncertainty reflects environmental changes that are outside the control of the organization, and potentially cause market instability (Williamson, 1975). Smallnumbers bargaining refers to the power parties have over a contract (Williamson, 1975). This can include the number of organizations or customers available to the party, or the routines or expectations that are expected from the agreement. Bounded rationality refers to the imperfect information surrounding the members or transactions, as well as the cognitive or emotional barriers to perfect knowledge (Williamson, 1975). Bounded rationality can result in opportunism, where one employee or community member is at the mercy of the other (Williamson, 1975). Bounded rationality and opportunism are affected by uncertainty and small numbers bargaining.

Though this theory revolves around economics, it can be applied to the relationship between the research organization and people with ADRD, where the transaction is participating in a research study. Uncertainty includes unawareness about the study process, or inability to learn more about research post-participation. Small bargaining is affected by the fact that it is completely voluntary to enroll, so the participant has the power to not join a study or drop out at any point. Bounded rationality includes the perspectives of the study, such as historical and perceived grievances against the research community. Opportunism includes that fact that the participant is at the mercy of the organization, for example, attending several appointments in person 
(Williamson, 1975). When these components, and the subsequent transaction cost, are too high to enter into a transaction, the organization benefits from a hierarchical structure to reduce uncertainties and small numbers bargaining. However, this organizational structure does not guarantee that the transaction cost will be at the optimal level to reach an agreement. Currently, the transaction cost is too high for many people with ADRD despite mitigating factors through the hierarchical structure, as evident though the lack of participation. Adjustments could be made within the research organization to further reduce the transaction costs for the future research participant.

Contingency theory: Another relevant organizational theory is contingency theory, which states that the best way to organize is dependent, or contingent, on the nature of the organization's environment (Galbraith, 1973). Lawrence and Lorsch (1967) specify that the structural function of the organizational subunits and the differentiation and mode of larger organization integration must align with the needs of the environment. Structured protocols can and should still exist within the research institution but should allow for flexibility as necessary to meet the organizational goals of increasing research participation and ability to restructure as necessary.

Mintzberg builds upon contingency theory to explore how bureaucratic organizations can develop environmentally-influenced rigid structures and create a more efficient organization. In Structure in Fives: Designing Effective Organizations, Mintzberg (1983) describes five categories of the organization as is illustrated in Figure 2.1. The operating core includes the frontline staff who perform direct support tasks integral to the organization's production of products and services. The strategic apex 
comprises the executive staff members who are responsible for the entire organization. They directly supervise staff, ensure the organization adheres to its mission, and meet the needs of external stakeholders who have control over the organization. The middle line includes middle management staff who perform tasks related to supervision of the operating core, supervision from the strategic apex, and communication through vertical, horizontal and external channels. The technostructure staff support production through training, designing and planning, but do not perform production work themselves. Lastly, the support staff focuses on tasks unrelated to production, but support the organization in other ways, such as legal counsel or cafeteria staff.

\section{Figure 2.1 Mintzberg's Model of Organizational Structure}

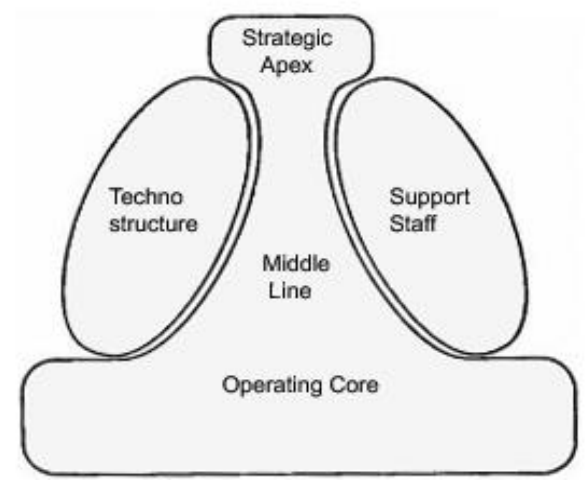

Source: Mintzberg, 1983, p. 11

Mintzberg (1983) further specifies five coordinating mechanisms. With mutual adjustment, coordination exists within the same level of hierarchy through informal communication. With direct supervision, higher level staff is responsible for lower level staff. Furthermore, there is the standardization of processes (specified tasks), outputs (specific results from task completion), and skills (specific knowledge required for job). 
Mintzberg also describes design parameters and structural parameters. Design parameters include role specialization, behavioral formulation, training and indoctrination; structural parameters include unit size, unit grouping, action planning and control systems. Every individual within the organization is internally influenced by these structures, mechanisms, and parameters.

Mintzberg's interpretation of contingency theory can be illustrated within the context of the proposed research study. Research assistants, usually at the forefront of recruiting, consenting, and enrolling individuals into studies, serve as the operating core. Investigators who design, secure funding, and lead the research serve as the strategic apex. Research coordinators manage and implement the studies, supervise research assistants, are supervised by investigators, and serve as middle line employees. IRB members, who ensure that research studies adhere to clinical and ethical regulations, serve as the technostructure. Research administrators who keep up to date with policies and procedures and handle back-end operations, also serve in this capacity. Lastly, support staff encompasses a large swath of research organization employees, including legal counsel, front desk staff, janitorial staff, and cafeteria workers.

Three of the five coordinating mechanisms -- mutual adjustment, direct supervision, and standardization of procedures -- are self-explanatory and do not need specific research examples. Specific outputs may include obtaining informed consent, study completion, and dissemination. Standardization of skills may include research assistant articulation of a study's purpose, benefits, and risks. Design and structural 
parameters, including friendliness and number of office employees, may differ from place to place but may influence the individual and the organization as a whole.

Given the tight hierarchy and number of regulations that must be adhered to (e.g. $\mathrm{NIH}$ and hospital-specific policies), it is difficult to completely redesign roles and structures of middle- and upper-level staff. However, the investigators could begin to include community members as external stakeholders whose needs should be considered in regard to the organization's mission. Research coordinators can communicate with external stakeholders, encouraging capacity building, and ensure that research assistants adhere to new internal policies based on external stakeholder needs.

At the operating core, several structures can be modified to increase clinical research participation, depending on the environment. For instance, the research assistant's skills could expand to incorporate personality traits and require greater comprehension of study background, methods and goals in order to explain to participants why being in the study is of value to them. Similar duties and responsibilities are found in service industries; operating core staff (e.g. customer service representatives) personalities and knowledge about products and organizational values frequently determine a customer's decision to conduct business with an organization. Similarly, research assistants are the first point of contact for potential research participants and are required to obtain informed consent. Without a friendly demeanor and comprehensive knowledge about the study, eligible individuals may be dissuaded from participating, wasting both the participant's and the organization's time, money, and energy. 


\section{Policy frameworks and theories}

Two policy frameworks, social construction framework and policy feedback theory (PFT), are applicable to ADRD clinical research participation.

Social Construction Framework: The social construction framework can be used to elucidate the power that people with ADRD wields in society. This theory posits that policies are contingent on policymaker and societal interpretations and values (Schneider, Ingram, \& deLeon, 2014). Policy designs (e.g. benefits, rules, problem definition) affect perceptions of target groups (e.g. people with ADRD), culture, and policymaking dynamics, culminating in future policy designs (Schneider et al., 2014). Benefits and burdens are allocated to target groups based on their political power, and their social construction (Schneider et al., 2014). Policies have consequential effects on society's attitudes towards target groups and the group's political participation (Schneider et al., 2014). At the same time, the policy effects influence both policymakers and society's decision-making and selection of future policy elements (Schneider et al., 2014). Changes in the target population's political power and social construction result in changes in public and personal perceptions, leading to policy change and further changes in the target population's material and interpretive effects, political power, and social construction (Schneider et al., 2014).

According to the framework, there are four categories of target groups, derived from political power and societal perceptions. The first category encompasses the Advantaged, who are positively viewed and powerful. The second includes Contenders, who are negatively viewed yet powerful. The third group, Delinquents, are negatively 
viewed and powerless. The fourth, Dependents, are positively viewed yet powerless. Examples of this framework in action are evident within the older adults' group and their transition from one category to another.

Older adults were considered Dependent for most of the $20^{\text {th }}$ century. Many policies that provided benefits, such as Social Security and the Older Americans Act, were met with little resistance because of the older adults' positive image and powerlessness in society (Hudson \& Gonyea, 2014). Estes' work on the Aging Enterprise highlights the Dependent category that older adults were grouped into prior to the 1980s, at which point the Reagan administration decentralized programs established by the Older Americans Act, and local and state governments took charge of resource allocation, planning, and service delivery (Estes, 1980). When resources were threatened, older adults became more politically active; as their advocacy groups became more vocal and gained more resources, they became one of the most powerful players in society and have successfully shifted to an Advantaged target population (Hudson \& Gonyea, 2014). In fact, twenty-five years after Estes's work was published, the director of the AARP encouraged businesses to focus their marketing efforts on the aging population, boasting that their independence and wealth could boost the economy if they are targeted with agefriendly products (Moody, 2004). Though the perceptions of the elderly population at large have shifted, older individuals with cognitive impairment still reside in the Dependents category. Policy designs and public perception haves deemed them worthy of benefits, but they are still viewed as powerless and unable to contribute to decision- 
making. This is evident within the research sector as study partner consent is required in order to move forward with participation.

Policy Feedback Theory: Policy Feedback Theory (PFT) can be used to conceptualize the motivation strategies for research participations. Skocpol (1992) coined "policy feedback" as the fourth of four hypotheses in her "Policy-Centered Analytical Framework of American Social Provisions." She suggests that policies implemented in Time Period One establish policy feedback through transformed state capacities (further defined by her as "administrative possibilities for official indicatives in the future and affect later prospects for policy implementation") and changes in social groups and their political goals and capabilities (Skocpol, 1992, p. 58). This policy feedback paves the way for policies implemented in Time Period Two. This is important because it captures the idea that a group's goals and capabilities both influence its preference for future policies.

One year later, Pierson expanded on Skocpol's work with his 1993 article "When Effect Becomes Cause.” His framework introduced three actors affected by feedback: a) government elites, b) interest groups, and c) mass publics, as well as the two mechanisms of feedback effects affecting these actors: a) resource and incentive effects and b) interpretive effects (Pierson, 1993). Resource and interpretive effects shape the actors' political behaviors while the interpretive effects convey information and meaning to them. Pierson's work is seminal because it transformed policy feedback from a term to a theory but left several gaps in the research. Aware of these gaps, he posited questions and 
future research directions that future PFT scholars could take. Two scholars who have made considerable leaps in the PFT realm are Suzanne Mettler and Kristen Goss.

Mettler's policy feedback for mass publics: Mettler (2002) has produced the most work on PFT to date, which largely focuses on civic engagement by community members, or what she refers to as mass publics. She established key components of civic engagement centered around Pierson's mechanisms of policy feedback, as shown in Figure 2.2.

Figure 2.2 Policy Feedback for Mass Publics: How Policy Affects Civic Engagement

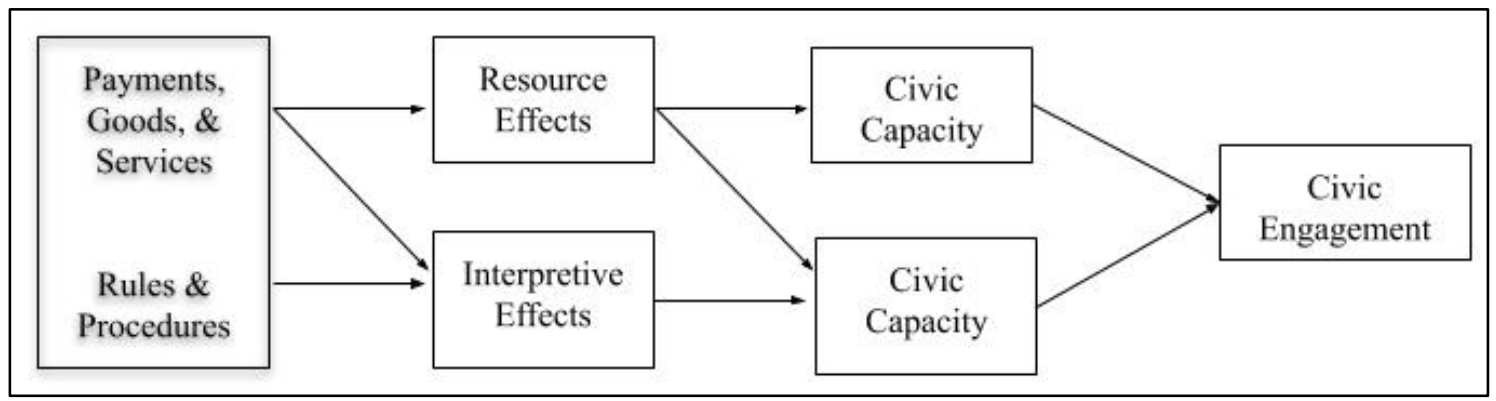

Source: Mettler \& Welch, 2004, p. 511

Mettler argues that payments, goods, and services establish resource effects, which affect civic capacity, or "aptitude, ability, or faculty to participate" (Mettler, 2002, p. 352). Resource effects also influence civic predisposition, defined as "the perceptions of their role in the community, their status in relation to other citizens and government, and the extent to which a policy has affected their lives" (Mettler, 2002, p. 352). Civic predisposition is also affected by the interpretive effects established through rules and procedures. Ultimately, both an individual's civic capacity and predisposition affect their civic engagement.

Mettler's framework can be applied to individuals with ADRD's likelihood of participation in clinical research. For instance, payments, goods, and services refer to 
potential benefits and risks of participating and contributing to society, education about and information on participation, and monetary compensation, all of which can affect both the ability an individual has to participate and their likelihood of participating. Their predisposition is also susceptible to rules and procedures, such as a rule to disseminate research updates and findings summaries to participants. Using Mettler's framework, policy changes can be made from the organizational level rather than the government level. A systematic review conducted by Newington and Metcalfe (2014) found several facilitators to increase participation rates. In addition to providing lay summaries to the participants, which has been discussed at length already, examples include: providing information on clinical studies at local community organizations, reducing the number of times a participant needs to travel to the study site, and having a good relationship with the participant's caregiver and clinician (Newington \& Metcalfe, 2014).

On its own, Mettler's framework provides a good starting point for evaluating policy effects on clinical research participation rates. However, it does not capture the effects from societal influence and organizational policies. In order to capture the effects that these variables have on clinical research participation among people with ADRD, a supplemental policy framework is needed.

Goss's multi-level policy feedback process: Goss's framework centers around the belief that society-level organizations are "vital to notions of individual's place in polity" (Goss, 2010, p. 122). She referred to these influences as "the organizational level" and developed her model based on Mettler's work. Goss argued that government policies 
influence the organization level in five ways, which in turn influence an individual's civic engagement (Goss, 2010, p. 120):

1. Government policies "structure the political orientation of civil society by stimulating the development of certain types of groups and strategies, while constraining others, with implications for the range of participatory opportunities afforded to individuals.”

2. Second, they "alter the capacity of civil society groups, including resources and political learning, to channel civic engagement towards non-political strategies of social improvement."

3. Third, government policies "affect the framing of strategies in a way that might influence mass attitudes about the optimal form that civic engagement should take."

4. Fourth, government policies "define civic membership" and "forge political community in ways that encourage rights-based advocacy over communitarian notions of public service.”

This framework is illustrated in Figure 2.3, below. 


\section{Figure 2.3 Multi-Level Process Feedback}

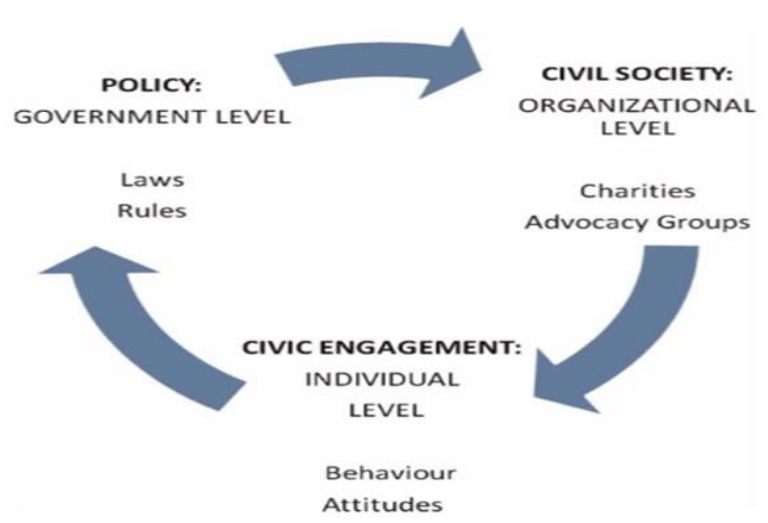

Source: Goss, 2010, p. 124

The numerous ways that policy shapes civic engagement through civil society also resonate with clinical research participation. A summary of how policies shape clinical research participation follows, based upon the literature review:

1. Government policy structures the organizational level: As previously mentioned, the U.S. Congress has allocated over 2 billion dollars to the NIH specifically for ADRD research for the period. The NIH in turn determines how many grants they award to ADRD research organizations, as well as how much funding is allocated per grant. The amount of funds and the number of grants accepted drastically influences the studies an organization can conduct, the length of time they have to conduct the research, the number of research staff they can hire, and the compensation they can offer a research participant. Additionally, NIH policies, such as inclusion policies and $21^{\text {st }}$ Century Cures Act (2016), affect inclusion and review criteria, data collection and analysis, and dissemination $(\mathrm{NIH}, 2017 \mathrm{c})$.

2. Government policy affects capacity of groups at organizational levels: In addition to allocating funding towards research, the federal government may also choose to 
increase or decrease grant funding for interest groups and nonprofit service providers, such as the Alzheimer's Association. Aside from offering educational and networking resources to ADRD individuals, caregivers, and professionals, they also provide a database for ADRD clinical research opportunities. Policies that decrease or underfund interest groups and nonprofits result in decreases in resources available to the organizations, and ultimately affect those who utilize these organizational resources.

3. Government policy frames the organizational level's predisposition: Interpretive effects from government policies not only affect individual predisposition to participate in clinical research, but also a group's predisposition to discuss or recommend opportunities to individuals. Organizations that want to increase ADRD clinical research participation are likely to discuss and recommend opportunities with people with ADRD. However, as previously mentioned, many individuals do not know where to obtain information about clinical research but expect to find out about research opportunities from their providers. Current policies hinder a provider's predisposition to discuss and recommend clinical research, such as a lack of time and reimbursement. Though clinicians can bill for a number of services, discussion of clinical research is not one of them (Fernandopulle, 2015).

4. Policies define membership, forge community relationships and promote advocacy: Just as belonging to a civic engagement group influences attitudes of making a difference in society, individuals who participate in clinical research are 
influenced in the same manner. Participants are proud to contribute to science and the quest for medical breakthroughs, even if they do not receive a therapeutic benefit themselves. Some research organizations, such as the OHSU Layton Aging and Alzheimer's Disease Center and the University of Washington Memory and Brain Wellness Center, offer community events and support groups for research participants or refer them to local government and community organizations, fostering relationships with other individuals with ADRD (OHSU Brain Institute, 2018; University of Washington, 2018). Moreover, both organizations and interest groups advocate for policies that promote research participation. The advocacy at the organizational level in turn provides individuals with greater capacity and predisposition to participate.

\section{Civic engagement}

Civic engagement embodies the notion that community members contribute individually and collectively in order to better their communities. Civically engaged individuals work together and develop their knowledge, skills, values, and motivation to make a difference in their community by promoting its quality of life (Ehrlich, 2000). Civic engagement in American society has gradually declined during the last half of the $20^{\text {th }}$ century (Putnam, 2000); however, when crises affect a community, such as a terrorist attack or natural disaster, civic engagement rates increase as measured by voting, discussing political affairs, and volunteering to mobilize community building efforts, including political campaigns and blood drives (Putnam, 2000; Sander \& Putnam, 2010). Civic engagement scholars have conducted research to determine ways to foster 
civic engagement in times of stability. They have found that organizations, governments, and policies influence the community's ability and motivation to participate in civic engagement activities (Bellah et al., 1991; Denhardt \& Denhardt, 2003; Vigoda-Gadot \& Cohen, 2004; Chandra et al., 2016). This section primarily describes civic engagement research conducted in the public administration realm but includes other examples from higher education and health services. Their compatibility with ADRD research is woven in throughout.

Civic engagement in public administration: In The Good Society, Bellah and his colleagues suggest that a public commitment to building a) institutions, b) family support, c) quality education, and d) self-respecting work empowers individuals to participate and serve as community stakeholders (Bellah et al., 1991). These values can also be translated to the clinical research realm: a public commitment to building supportive regulations and initiatives for research institutions, supports for people ADRD and their caregivers, quality education on clinical research history, purpose, and opportunities, and treating research participants as respective, collaborative partners could empower individuals to act as stakeholders and participate in clinical research. Thus, a civic engagement framework is relevant to this research.

Similarly, public administration scholars Vigoda-Gadot and Cohen (2004) suggest moving away from the idea of viewing community members as subjects or voters; instead, the government should treat community members as customers and be responsive to their needs. They suggest that the best way to do so is by forming and maintaining government-community partnerships that include greater cooperation with 
local private, nonprofit and volunteer organizations, local initiatives that foster democratic education and participation and involvement, and community members' innovative ideas for problem-solving (Vigoda-Gadot \& Cohen, 2004). These partnerships can enhance community involvement in the government and "generate(s) commitment to a healthy public service, proper understanding of what is right and what is wrong in managing public organizations, and education towards constructive participatory democracy" (Vigoda-Gadot \& Cohen, 2004, p. 14).

Denhardt and Denhardt (2003) advocate for a public administration governing style that encourages civic engagement by including community members as active stakeholders, distinguishing themselves from older styles such as "old public service" and "new public management" where community members are treated as beneficiaries or customers. In "old public service", politics is separated from administration, and public organizations are organized in hierarchies to ensure "greatest possible efficiency in their operations," with limited citizen involvement (Denhardt \& Denhardt, 2003, pp. 6-7). From there stems "new public management" which relies on market mechanisms (e.g. competition, contracting) and is supported by rational choice and managerialism, in which business and public sector success relies on management quality and professionalism (Denhardt \& Denhardt, 2003). A newer wave of public administration, known as "New Public Service", positions community members and public officials to work together to define and to address common problems in a cooperative and mutually beneficial way. The "New Public Service" incorporates the following seven lessons (Denhardt \& Denhardt, 2003): 
1. Serve citizens ${ }^{1}$, not customers: The public interest is the result of a dialogue about shared values rather than the aggregation of individual self-interest. Therefore, public servants do not merely respond to the demands of "customers," but rather "focus on building relationships of trust and collaboration with and among citizens" (Denhardt \& Denhardt, 2003, p. 45). Though self-interest plays a role, the shift to thinking democratically has greater benefits for both society and its individuals.

2. Seek the public interest: In the New Public Service, public servants aim to encourage community members to think about their shared values with each other, further develop those values, and increase their capacity to help the public interest. To do so, trust must exist between government and community members.

3. Value citizenship over entrepreneurship: In the New Public Service, it is not enough for the administrators to create and implement policies; they must also continually engage with the community to recreate and rearticulate public values. Community members actively participate by defining and managing the processes.

4. Think strategically, act democratically: In the New Public Service, community engagement and community building are part of implementation. Government leaders and public servants must first show that they are open, accessible, and

\footnotetext{
${ }^{1}$ Denhardt \& Denhardt use the term "citizens"; I choose to use the term "community members" as it recognizes and includes non-citizens living in the community. When direct quotes are used, the term "citizen" will appear.
} 
responsive, and can create opportunities that incentivize the community to collaborate on various programs.

5. Recognize that accountability isn't that simple: In the New Public Service, public administrators are responsible for taking "political authority seriously" in addition to their "obligations with respect to the duties of others as well as the role of the responsible public servants in policy formulation and implementation" (Denhardt \& Denhardt, 2003, p. 134). This means that leaders are elected or appointed to their positions based on their values and expertise, however, their policy decisions must result from a participatory process that includes administrative supervisors and community members. Ultimately, public servants are accountable to everyone.

6. Serve rather than steer: In the New Public Service, leaders share their responsibilities, are value-based, and recognize that their role is to serve the community, not own them. Public administrators must view their role as responsible participants who share power, work with people, and broker solutions.

7. Value people, not just productivity: In the New Public Service, "public organizations and the networks in which they participate are more likely to be successful in the long run if they are operated through processes of collaboration and shared leadership based on respect for all people" (Denhardt \& Denhardt, 2003, p. 155). Rather than attempting to control behavior, management processes are established and modified based on the needs, values, interests and input from the people involved. Additionally, managers must "encourage, model, and enact 
our commitment to democratic ideals and the trust of others" (Denhardt \& Denhardt, 2003, p. 165). This means that in order to expect community members to be committed to democratic ideals and trust their government and community, the government must include them as partners and provide the same trust and respect that they strive for.

Civic engagement scholars have also identified the flawed assumption that some community members will forget past negative, and often egregious, relationships to forge new ones that may produce a benefit (Bellah et al., 1991; Denhardt \& Denhardt, 2003). This is also evident in the clinical research sector. For example, members of the African American community are more likely to be diagnosed with ADRD, yet less likely to participate in clinical research for previously discussed reasons (Danner et al., 2011; Gilmore-Bykovskyi et al., 2019). However, when clinical research organizations take time to establish a relationship that builds trust and respect and provide full information about study opportunities in a clear, understandable manner, African Americans are more likely to enroll in their studies (Gifford et al., 2002; Danner et al., 2011; Inungu et al., 2017; Gilmore-Bykovskyi et al., 2019).

Civic engagement in higher education: Civic engagement scholarship has also emerged from the field of higher education. Traditionally, higher education implored and motivated students to expand their minds and improve their community. In the mid- $20^{\text {th }}$ century, many universities began to offer or require service-learning courses, where students apply what they have learned in the classroom directly to the community, most often by working with an organization designed to address a community need (Lounsbury 
\& Pollack, 2001). Additionally, college campuses became hotbeds for student protests of policies and norms that negatively impacted their community (Lounsbury \& Pollack, 2001).

By the turn of the century, scholars asserted that higher education had shifted towards courses and student opportunities that may result in better, higher-paying jobs rather than community engagement and societal benefit (Kerr, 1994; Boyer, 1996; Battistoni \& Longo, 2011; Saltmarsh \& Hartley, 2011). Universities' ideas of knowledge were viewed through an academic lens, impeding a view of the public's understanding (Mathews, 2000). Furthermore, institutionalization gradually shifted control and resources away from students to people with formal power and bureaucratic authority on campus (Lui, 1996). Saltmarsh \& Hartley (2011) identify several ways in which higher education has undermined its democratic processes. First, faculty pursued narrow specialties, which advance theories but do little to address social problems. Second, corporatization of universities had created a dissonance between programs and shaping other practices. This means that as majors and programs became more specialized towards career aspirations, there became less of a focus on general education, consequently segregating students by major. Third, a crisis in undergraduate teaching and decontextualized learning occurred, where many professors unduly influenced students without providing relevant experiences. Fourth, there was a sense of civic disengagement and hibernation in that universities did not teach students to be citizens. Lastly, there was a lack of "general reciprocal learning;" instead of being guided by Freire's advocacy of shared learning, power dynamics between faculty and students widened, and many 
faculty arrived at the idea that student knowledge was not legitimate as compared to faculty knowledge (Botye, 2008).

Since then, academic institutions have worked to revert back to the civically engaged ways of the mid-century. For example, Widener University in Pennsylvania requires their physical therapy students to engage in service-learning by providing blood pressure screenings to low-income seniors and working pro bono at a local safety clinic (Palombaro et al., 2017). Compared with students who did not participate in service learning, the physical therapy students became more civically minded over the course of their program (Palombaro et al., 2017). Other higher education institutions have remained civically engaged. For example, the West Philadelphia Improvement Corps (WEPIC), a school-community-university partnership, has been in existence since 1985 (Benson, Harkavy \& Puckett, 2011). In this partnership, the University of Pennsylvania works with two local high and middle schools to motivate and teach youth to revitalize their communities. Over the past three decades, they have established adult education classes, job training, drug prevention programs, and community outreach (Campus Connect, 2016). The WEPIC partnership exemplifies another concept of civic engagement in higher learning, knowledge mobilization, discussed in the Culture of Health section.

Similarly, Portland State University (PSU) has established sustainable partnerships with many community organizations and members since the mid-1990s (Holland \& Gelmon, 1998). These partnerships are sustainable in part due to mutually agreed-upon goals, an agenda that is mainly controlled by the community, success that is 
measured by both university and community standards, and consistent capacity building and evaluation (Holland \& Gelmon, 1998).

Saltmarsh \& Hartley (2011) believe that for higher education to shift towards a civically engaged approach, local stakeholders must be included and values of “inclusiveness, participation, risk sharing and reciprocity in public problem solving, and an equality of respect for the knowledge and experience that everyone involved contributes" need to be invoked (Saltmarsh \& Hartley, 2011, p. 18). Battistoni \& Longo (2011) suggest that students need to be part of the conversation, but also suggest a change in the way that civic engagement is conceptualized, taught, and practiced. They suggest three values be added to the traditional civic engagement framework. The first is the student voice, which allows students to define civic and political engagement for themselves and contribute to campus decision making on the issues that affect and matter to them. The second is the practice of direct participation by providing or finding opportunities to practice civic engagement planning, development, and implementation. The third is motivation. These authors stress that it is not enough to have the skills to become civically engaged; students need to be motivated to become civically engaged. To advance these student-centered models of civic engagement, universities must empower students to define civic issues and public work, create opportunities for longerterm engagement, and reconstitute faculty-student relationships where faculty can be seen as mentors and students can be seen as colleagues. The inclusion of these three values could lead to increased voting and participation in democratic society (Battistoni \& Longo, 2011). 
As in higher education, the research organization has similarly undermined democratic processes. Investigators have specialties, advancing knowledge in their narrow field without addressing barriers to research participation (Kramer et al., 2012. The research organization has also become corporately influenced. Different organizations offer different studies, restricting geographic access to potential participants, studies typically recruit younger white males, and bureaucracy exists within the research organization (Kramer et al., 2012; Freidman et al., 2016; Mahon et al., 2016; Banzi et al., 2016; Perez-Stable, 2018; NIA, 2018). Research staff may unduly influence potential participants in their decision to participate because of their demeanor towards and relationship with potential participants (King et al., 2007; Penberthy et al., 2012; Brown et al., 2013). Further, until recently, research organizations did not engage with or seek out the expertise of research participants, resulting in a lack of shared knowledge (Williams et al., 2011; Selby, Beal, \& Frank, 2012; Johnson et al., 2015; NIA, 2018). However, like higher education, research organizations are currently working towards a more civically engaged approach to research through community involvement. As previously mentioned, the NIH asked for public comment on ways to improve research participation. Additionally, the NIH has promoted funding for investigators who seek to work with communities and build partnerships to improve clinical research diversity, recruitment, and retention (NIH, 2018). Involving the voices of people with ADRD may help define what research issues are most imperative to them and allows them to be involved in the decision-making process. The practice of direct participation may occur by allowing them to be involved in the planning, design, and implementation 
processes of the research study. Finally, the research organization can motivate individuals with ADRD by empowering them to play an active role in research, creating long-term opportunities to facilitate engagement, and enhancing the relationship between the research organization staff and people with ADRD where they would be treated as colleagues.

Civic engagement in health services: Since the turn of the century, civic engagement approaches have become more utilized in the health services. The most prominent feature of civic engagement in health services is the Patient-Centered Outcomes Research Institute (PCORI). PCORI created by Congress through the Affordable Care Act in 2010 to ensure that comparative effectiveness research "guided by patients, caregivers, and the broader healthcare community" and that research is being conducted to determine choices and outcomes that are most meaningful to patients (Selby et al., 2012, p. 583; PCORI, 2017). Rather than simply enrolling patients into community engaged research studies, patient-engaged research views patients and family members as stakeholder and members of the research team. Some of their tasks include: a) identifying topics and formulating research questions; b) identifying study populations and choosing interventions, comparers, and outcomes; c) developing and implementing strategies for recruitment and retention; d) conducting and monitoring the study; e) analyzing data and interpreting findings; and f) disseminating results (PCORI, 2017).

A review of patient engagement studies conducted by Esmail and her colleagues (2015) found that patient engagement leads to better quality research, patient empowerment, increased translation and dissemination and results, develops trust 
between patients and researchers, and continued engagement in research. However, they also noted a gap in evaluations of patient engagement, particularly for long-term outcomes (Esmail, Moore, \& Rein, 2015). Community Research Partners, an online database, connects research organizations and patients together, and provides resources for research organizations on a variety of topics, including community-engaged research, health disparities, and translation and dissemination (Community Research Partners, 2012).

Similar to public administration and higher education, civic engagement has been increasingly incorporated in health services at the state and clinic level. When Oregon developed its coordinated care organization (CCO) system, it included community voices at the start of the design process. In thirteen months, the state held over 90 public forums across the state to discuss its plans, learn what each community wanted from a state healthcare system, and how this could be achieved in their local area (Wentz, 2017). Each $\mathrm{CCO}$ has a community advisory board, comprised of a Medicaid-insured majority to ensure the patient voice is heard and included within the policymaking process (Stout et al., 2017). In addition, community members serve on a variety of other state health care advisory boards and all are invited to attend open meetings (Tillman, 2017). Furthermore, the state Health Equity Policy Committee includes and ensures that voices of underserved community members, such as people of color, immigrant and refugees, and people from rural parts of the state, are heard and incorporated into Oregon's health policies (Tillman, 2017). 
Civic engagement is also becoming more common at the clinic level. Many clinics in Oregon, especially those that participate in the state's Patient Centered Primary Care Home (PCPCH) program, establish patient and family advisory councils (Gelmon, Wallace, Sandberg, Petchel, \& Bouranis, 2016). In these councils, clinic patients and family members provide feedback on both positive and negative experiences and offer suggestions to improve workflow processes, quality, and care (Gelmon et al., 2016). When creating this program, the Oregon Health Authority established a council comprised of providers, patient advocates, insurers, policymakers, and major medical societies to help determine standards that clinics should be required to meet (Smith \& Merrithew, 2017). Their interdisciplinary input crafted the program to be communityfocused by, for example, working with community partners to determine the best neighborhood resources to manage their patient populations' diabetes (Smith \& Merrithew, 2017). Additionally, as previously mentioned in the LTSS section, shareddecision making is frequently invoked, so providers, patients, and family members (if the patient includes them) collaboratively develop the patient's care plan. Providers utilize their medical expertise to determine what the best course of action is, while the patient and family bring the expertise of determining what they are willing and/or able to do. Furthermore, shared decision-making and deliberation often includes a discussion of community resources, such as social services, that are easily accessible to the patient and their family (Gelmon et al., 2016). These additional collaborations enhance the capacity across the individual, the healthcare system, and other social and community sectors. 
How health services incorporate civic engagement, and how it can be applied to ADRD clinical research, is discussed in more detail in the next section.

Many of the elements of civic engagement and recommendations for enhancing engagement, including empowerment, enhanced communication, reaching out to community members, and shared decision making and collaboration, not only apply to research participation, but are also frequently suggested within the peer-reviewed literature, as previously cited. These elements and recommendations are also some of the features with the Culture of Health Action Framework. Though aimed towards health services, this framework could be applied to the research sector and is also relevant to this research.

\section{The Culture of Health Action Framework}

The Culture of Health Action Framework was developed by the Robert Wood Johnson Foundation in 2014 and encourages collaboration across the health and social sectors (Weil, 2016). In the framework's conceptualization, health providers partner with local nonprofits and private organizations; these partnerships then work with communities to define their needs and the measures that matter most to them to improve population health, wellbeing, and equity (Weil, 2016). Partners then design and redesign programs, even if they had been previously successful, to better meet community needs and achieve the Culture of Health Action Framework goals of improving community health, wellbeing, and equity (Weil, 2016). When compared with other communities, those with high-density multi-sector networks supporting health improvement activities have better health outcomes (McCullough \& Leider, 2016). 
Figure 2.4:

\section{Figure 2.4: The Culture of Health Action Framework}

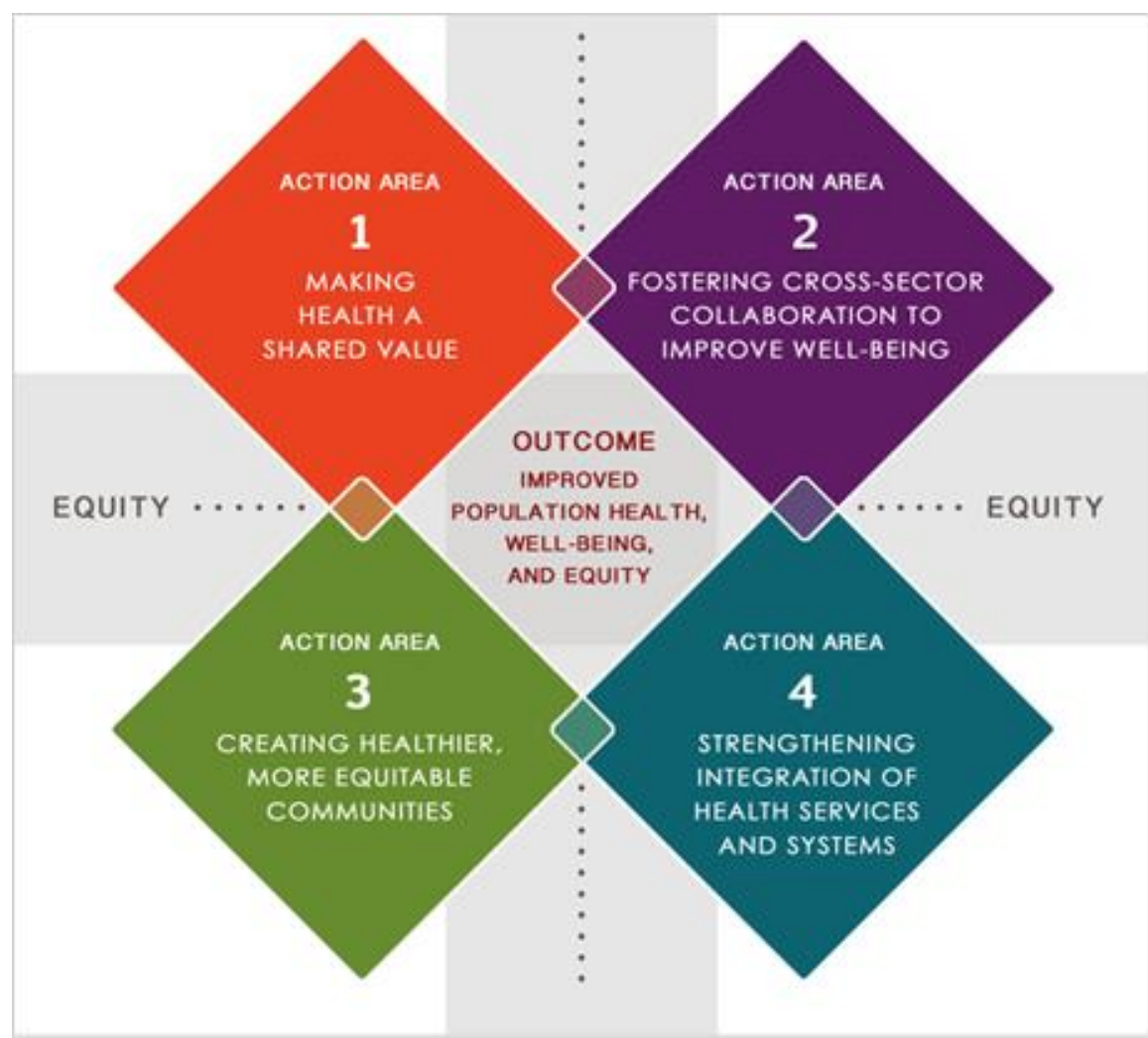

Source: The Robert Wood Johnson Foundation, 2016

Action Area 1. Make health a shared value: In their focused article on this action area,

Chandra and her colleagues discuss how individuals and communities view their health and wellbeing, and debate whether the community is expected to advocate for health improving policies, environments, and services (Chandra et al., 2016). They articulate that there are several challenges to a Culture of Health in the United States. This includes conversations that focus on cost control and containment rather than health and wellbeing, frameworks that do not consider social networking and social movements as 
policy issues, and the lack of equity as an analyzed metric (Chandra et al., 2016). In order to combat these three challenges, the Robert Wood Johnson Foundation recommends three changes be made before communities can view health as a shared value. First, there must be a shift in mindsets and expectations of what individuals consider health and wellbeing, with an emphasis on group benefits and opportunities. Individuals must view health as shared and expected, not a function of economic privilege. Mindsets and expectations can shift by stressing the benefits of equitable health opportunities, but also require group-level empowerment in order to push for social change (Chandra et al., 2016). Secondly, health as a shared value requires a sense of community identity. Whether it is perceived or not, individuals and community are interdependent given their sense of community membership, belonging, and shared experiences. When community members recognize and act upon their solidarity, they are able to better mobilize and enhance their ability to thrive, be healthier, and drive change. Lastly, making health a shared value requires the knowledge of civic engagement skills, values, motivation, and opportunities within that community (Chandra et al., 2016).

Many individuals affected by medical conditions, including ADRD, want to participate in related clinical research for both altruistic and potentially therapeutic reasons; however, many are unaware of how to seek or receive information about research opportunities (English et al., 2010; Law et al., 2014; Mahon et al., 2016; Anderson et al., 2018). As previously mentioned, many expect to hear about opportunities from their trusted provider or community leader; however, they are more likely to hear of opportunities from sources that they have less trust in, such as radio and 
newspaper (English, et al., 2010; Law et al., 2014; Mahon et al., 2016; Anderson et al., 2018). The literature on political participation, blood donation, and knowledge transfer also offers factors that increase motivation (Lee, Piliavan, \& Call, 1998; Brady et al, 1999; Law et al., 2003; Sojka \& Sojka, 2008; Beyerland \& Bergstrand, 2016).

Individuals are more likely to become politically involved if they frequently discuss political issues and freely share their views (Beyerland \& Bergstrand, 2016). They are also more likely to participate in civic activities if they are being asked by their partner, parent, child, or a member of their community or religious congregation (Beyerland \& Bergstrand, 2016). Having a close relationship with a blood donor also facilitates blood donation; for these reasons, Sojka and Sojka suggest the "bring a friend" approach, which may be translated to research participation (Lee et al., 1999; Sojka \& Sojka, 2008). Given concerns of fear, anxiety, and pain during the blood donation process, donors need more personal reasons to compensate for the risk, including general altruism or knowing a blood recipient (Lee et al., 1999). Additionally, people who identify themselves as blood donors are more likely to continue being a blood donor (Lee et al., 1999; Sojka \& Sojka, 2008). Similarly, individuals who participate in research tend to do so for altruistic reasons and are more likely to view themselves as research participants (Owen et al., 2013; Friedman et al., 2015; Gollhofer et al., 2015; Anderson et al., 2018).

Knowledge transfer is another helpful way to inform communities about research studies. Information from researchers is translated to communities, which requires determination of what information is important to the community, who a credible and 
trusted messenger would be, how the message should be transmitted, and the effects the message should have (Lavis et al., 2003). Knowledge transfer can be used to directly solicit community members to participate, which increases motivation for political involvement, blood donation, and research participation (Brady, Schlozman, \& Verba, 1999; Law et al., 2014; Beyerland \& Bergstrand, 2016).

Action Area 2. Foster cross-sector collaboration to improve well-being: Three drivers of cross-sector collaboration, as articulated in the Culture of Health Action Framework include: a) number, breadth, and quality of successful cross-sector partnerships; b) adequacy of investment in these partnerships; and c) adoption of policies to support the partnerships (Towe et al., 2016). High quality cross-sector partnerships occur through stakeholder commitment, community buy-in, and advocacy to establish meaningful collaboration among stakeholders. Stakeholders should research returns on investments, how funding mechanisms can be shaped to address equity, how partners will engage, and how partnership sectors apply and benefit from these collaborations (Towe et al., 2016). Initiatives to create cross-sector collaborative can be formulated at local, state, and federal levels and require collaboration across multiple agencies.

The Satellite program in St. Louis exemplifies how high-quality cross-sector partnerships can lead to participatory processes and outcomes. A federal grant from the NIA in 1992 provided the financial initiative to establish the program, and over several years, they developed partnerships with local clergy, the Alzheimer's Association, government offices, senior centers, libraries, and African American community organizations (Williams et al., 2011). These partnerships were sustained through 
numerous types of investments, including federal grants and philanthropic contributions (Williams et al., 2011). Policies were created to maintain their partnerships and achieve their goals, such as the establishment of an African American ADRD research advisory board in 2004 (Williams et al., 2011). Satellite has achieved their primary goal of removing research participation inequity among the African American community in St. Louis; their success has led to the development of similar programs in California, Wisconsin, Kentucky, and New York (Williams et al., 2011; NIA, n.d.).

The higher education and civic engagement literature offer best practices for fostering cross-sector collaboration. With knowledge mobilization, partnerships are "shaped by the organizations engaged in the production, brokerage, dissemination, and uptake of the research knowledge, [and] in turn, are embedded in broader social contexts that influence priorities, opportunities, and demands for research knowledge" (Sa, Li, \& Faubert, 2011, p. 504). It is collaborative and facilitated by organizational resources and norms. Systematic collaboration among partners, faculty incentives and rewards, and programmatic changes enhance the strength of the partnerships and the influence of knowledge mobilization on resource allocation and opportunities for the community. The NIH's May 2018 announcement of funding opportunities to promote diversity, recruitment, and retention through collaborative partnerships may serve as a gateway to knowledge mobilization in ADRD research. Receiving an NIH grant is a financial and academic incentive for investigators, who may then implement programmatic changes within their research organization that establish or strengthen collaborative partnerships 
as well as influence resource allocation and opportunities based on the expertise of the participants and study partners.

Civic engagement scholars state that partnership requires greater cooperation with volunteer, community, and private-sector organizations, as well as state and municipal initiatives that foster values of education, participation, and citizen involvement (Bellah et al., 1991; Vigoda-Gadot \& Cohen, 2004). These values empower individuals to become stakeholders in their community, increasing participation. Treating community members as stakeholders is imperative to fostering relationships and future collaboration; current and potential participants cited failure to disseminate research results and updates as a reason for not participating in future opportunities (Sood et al., 2009; Law et al., 2014; Mahon et al., 2016).

Action Area 3. Create healthier, more equitable communities: Improving physical environments, social and economic conditions, and policies that facilitate coalitionbuilding and improvement projects will enhance opportunities for healthier and equitable communities (Dubowitz et al., 2016). However, the Robert Wood Johnson Foundation suggests that progress in this action area needs to be made before new processes can be implemented. First, the complexity of causality of poor health outcomes must be recognized. Dubowitz and colleagues exemplify this challenge with a grocery store: simply adding a grocery store in a neighborhood may improve the neighborhood's quality, but not improve healthy eating (Dubowitz et al., 2016). Individuals living in the neighborhood may prefer to purchase food from other locations, may not be able to afford the groceries, may not have transportation to get there, or there may not be 
adequate structural supports, such as sidewalks or street lights, that would allow them to safely walk to the store. Second, new governance structures may need to be created to sustain action. Opportunities exist in cross-silo government approaches but rely on elected and appointed executive officials to identify, develop, and maintain them. If officials are not supportive, opportunities may not come to fruition. Last, creating healthier and more equitable communities requires mobilizing broad coalitions amid political polarization (Dubowitz et al., 2016). Problem framing plays a significant role in decision-making, and opportunities may result from framing social determinants as equally complementary to the role of individual choice in health outcomes, rather than viewing them as siloed factors. When health sectors partner with community members as well as local government and organizations, all stakeholder may begin to recognize the complex causality of health outcomes due to social determinants and individual choice and develop solutions that align with the community's needs.

Similarly, the reasons why people with ADRD chose to participate in clinical research or not are complex as participation requires both capacity and predisposition to participate. Just as adding a new grocery store in a neighborhood does not automatically improve health outcomes, offering a new research opportunity does not necessarily improve recruitment and retention. As previously discussed in the clinical research section, there are a myriad of factors that impede or facilitate an individual's ability and motivation to participate in research. Collaboration among the research sector, people with ADRD, and other relevant stakeholders is the best way to identify barriers, 
facilitators, and solutions to increase participation (Grill \& Galvin, 2014; Mahon et al., 2016; Kremer, 2017).

The New Public Service public administration style may serve as an example as it encourages collaboration among community members and public officials to define and address common problems, including physical environment and social and economic conditions, in a cooperative and mutually beneficial way (Denhardt \& Denhardt, 2003). Public servants aim to motivate community members to think about their shared values within their community, further develop those values, and increase their capacity to help each other; with every open conversation, steps are taken to establish a trusting relationship between the community and the public servants (Denhardt \& Denhardt, 2003). In the New Public Service, public servants view their role as responsible participants who share power, work with people, and broker solutions (Denhardt \& Denhardt, 2003). Values are continuously redefined and revised by the community, and public servants implement policies based on those continually redefined values. The Culture of Health Action Framework also recognizes this necessity; stakeholders from health and other sectors work with the community to identify their needs and values and develop programs that align with them. Programs are designed and redesigned as needed, and like New Public Service, capacity, trust, and relationships among all stakeholders are strengthened as the partnerships share power, work together, and develop solutions.

New Public Service can influence the relationships among community members and research organizations. Organizations often assume that individuals will enter a relationship if it is beneficial; however, if there is a history of bad relations or culture, 
they will avoid it, as exemplified in the previous discussion on African American participation in clinical research. However, if the organization takes the time to establish a relationship to build trust and respect and provide full information about study opportunities in a clear, understandable manner, they are more likely to participate in research (Danner et al., 2011, Frew et al., 2011; Grill \& Galvin, 2014; Mahon et al., 2016, Inungu et al., 2017). Ultimately, removing the power dynamics and valuing the expertise that people with ADRD have reduces the inequities among stakeholders, which will lead to identifying problems and solutions that are needed to reduce inequities, remove barriers, and improve ADRD clinical research participation. The PatientCentered Outcomes Research Institute (PCORI) funds 13 studies that focus on dementia and cognitive impairments (PCORI, 2020a). One of these studies includes people with ADRD as stakeholders. LiveWell, a dementia service provider in Connecticut (PCORI, 2019), received a two-year award to increase the capacity of people living with dementia and their caregivers and reduce barriers to their engagement in patient-centered research (LiveWell, 2018). Although this is a sign of a shift in recognizing the expertise of people with ADRD, the small number of dementia and cognitive impairment patient-centered studies pales in comparison to the 89 PCORI-funded studies focusing on cancer or the 133 studies focusing on mental and behavioral health (PCORI, 2020b; PCORI 2020c). Action Area 4. Strengthen health services and systems: Drivers that strengthen the integration of health services and systems include access, balance and integration, and consumer experience and quality. Access to health services and systems requires reducing and/or removing barriers due to cost and insurance, language, transportation, 
office hours and culture (Martin et al., 2016). Balance refers to how resources are prioritized and allocated between and within health and social sectors, whereas integration refers to the meaningful connections that are made between sectors (Martin et al., 2016). Consumer experience and quality is relevant to the Triple Aim (Berwick, Nolan, \& Whittington, 2008) as one goal of the Culture of Health Action Framework is to improve the community's health and experience while also lowering costs (Martin et al., 2016).

Integrating medical care, public health, and social services systems can connect community members to appropriate and timely information, supports, preventive services, and formal healthcare to improve health and wellbeing (Martin et al., 2016). Progress in this action area requires identifying and utilizing models that focus on improving access to healthcare and removing barriers such as language, office hours, transportation, and lack of cultural competence. Discussions on resource allocation and priorities, and their effects on experience and quality, are also necessary (Martin et al., 2016).

Individuals report being more open to clinical research participation if organizations are accommodating by offering flexible scheduling, fewer in-person visits with opportunities for video conferencing, and free or subsidized modes of transportation (Danner et al., 2011; Law et al., 2014; Friedman et al., 2015; Mahon et al., 2016). Research organizations often ignore the barriers encountered by residents of rural communities, who reside too far from the organization to attend appointments, and members of non-English-speaking communities, who are excluded by virtue of not 
speaking English (King et al., 2007; Friedman et al., 2013; Grill \& Galvin, 2014; Mahon et al., 2016). Denying access to research opportunities demotivates members of these communities who have ADRD.

The primary care medical home $(\mathrm{PCMH})$ literature offers examples of how health sectors can become more accessible, integrated and balanced, and improve consumer experience and quality, ultimately strengthening health services and systems. In Oregon, where over $70 \%$ of the primary care clinics participate in the $\mathrm{PCPCH}$ program, many provide office hours outside of the typical 9-5 workweek, and all are required to have a 24/7 telephone line (Smith \& Merrithew, 2017; Gelmon et al., 2018). Clinic leaders report translating materials and hiring bilingual and/or multicultural staff to better serve their patient populations, and care coordinators build repositories of community resources or work with other local health and social sector organizations to address other structural barriers (Gelmon et al., 2018). Coordinated care organizations highlight ways to balance resources between and among health and social sectors, as well as the meaningful connections between and among them. In Oregon, CCOs serve the Medicaid population and ensure that every consumer has access to physical, mental, and dental health providers in their local community (Oregon Health Authority, n.d.). Since CCOs work to foster preventive health, they partner with local government, nonprofit, and private organizations to provide resources to patients. For example, CareOregon, a Portlandbased managed care organization, partners with local nonprofits and private organizations to offer its members free nutritious food delivered to their home, cooking classes, and nutrition literacy classes (CareOregon, 2017). Individuals who participate in this program 
have reported that the program has made a real difference in their lives (CareOregon, 2017). Though there are no studies that have evaluated the PCPCH program from the patient and family perspectives to date, clinic leaders perceive a positive difference for their patients (Gelmon et al., 2018).

Conclusion: The Culture of Health Action Framework and its action areas could be useful for articulating strategies for motivating factors for research participation. Table 2.3 highlights the twelve drivers and their applicability to ADRD clinical research.

Table 2.3 Operationalization of the Culture of Health Action Framework

\begin{tabular}{|c|c|}
\hline Drivers & Description and relevant examples \\
\hline \multicolumn{2}{|r|}{ Action Area 1: Health is a shared value } \\
\hline $\begin{array}{l}\text { Mindsets and } \\
\text { expectations }\end{array}$ & $\begin{array}{l}\text { An emphasis is placed on group benefit and expectations; } \\
\text { requires group-level empowerment to advocate for } \\
\text { resources (Chandra et al., 2016). Per the Belmont Report, } \\
\text { individuals may participate in clinical research to benefit } \\
\text { society, even if they do not benefit directly (The } \\
\text { Commission, 1979). This is true in clinical research, and } \\
\text { many report participating for the benefit of the community } \\
\text { (English et al., 2010; Law et al., 2014; Mahon et al., 2016; } \\
\text { Anderson et al., 2018) }\end{array}$ \\
\hline Sense of community & $\begin{array}{l}\text { Community members are interdependent, even if they do } \\
\text { not realize they are a part of a community (Chandra et al., } \\
\text { 2016). When they do recognize themselves as a } \\
\text { community, their ability to motivate and drive change is } \\
\text { enhanced (Denhardt \& Denhardt, 2003; Chandra et al., } \\
\text { 2016). This is evident by the advocacy efforts in cancer } \\
\text { survivors and the LGTBQ+ community, who were not } \\
\text { previously recognized due to societal and self- } \\
\text { stigmatization. }\end{array}$ \\
\hline Civic engagement & $\begin{array}{l}\text { To become civically engaged, an individual must have the } \\
\text { resources, skills, values, and knowledge of opportunities } \\
\text { (Denhardt \& Denhardt, 2003; Vigoda-Gadot \& Cohen, } \\
\text { 2004; Chandra et al., 2016). Similarly, for individuals to } \\
\text { participate in clinical research, they must be aware of } \\
\text { opportunities to participate in research, hold participation } \\
\text { in clinical research as an important value, meet the } \\
\text { eligibility criteria, and have the resources (e.g. time, }\end{array}$ \\
\hline
\end{tabular}




\begin{tabular}{|c|c|}
\hline & $\begin{array}{l}\text { transportation) to be able to participate (Grill \& Galvin, } \\
\text { 2014; Mahon et al., 2016; Anderson et al., 2017). }\end{array}$ \\
\hline \multicolumn{2}{|c|}{ Action Area 2: Fostering cross-sector collaboration to improve wellbeing } \\
\hline $\begin{array}{l}\text { Number and breadth } \\
\text { of partnerships }\end{array}$ & $\begin{array}{l}\text { This requires stakeholder commitment, community buy-in, } \\
\text { and advocacy (Towe et al., 2016). Examples of these } \\
\text { partnerships include the Alzheimer's Association, } \\
\text { PreSERVE, OHSU Community Resources Coalition and } \\
\text { Liaisons, and the Satellite program in St. Louis (Williams } \\
\text { et al., 2011). }\end{array}$ \\
\hline $\begin{array}{l}\text { Investment in cross- } \\
\text { sector collaboration }\end{array}$ & $\begin{array}{l}\text { This requires knowledge of the return of investments, how } \\
\text { funding mechanisms address equity, and how partnerships } \\
\text { apply, engage, and benefit (Towe et al., 2016). This can be } \\
\text { applied through immediate knowledge transfer to all } \\
\text { interested community members, research organizations, } \\
\text { health and social sectors, and advocacy groups (Sa et al., } \\
\text { 2014; Sood et al., 2009; Law et al., 2014; Mahon et al., } \\
\text { 2016). If applying for an NIH grant for an ADRD clinical } \\
\text { research study, investigators must now include all of this } \\
\text { information (NIH, 2017c). }\end{array}$ \\
\hline $\begin{array}{l}\text { Policies that support } \\
\text { collaboration }\end{array}$ & $\begin{array}{l}\text { These policies can occur at local, state, or federal levels } \\
\text { (Towe et al., 2016). Examples include the NIH's } \\
\text { recruitment, retention, and diversity grant, Oregon Health } \\
\text { Authority's Health in All Policies, and Multnomah } \\
\text { County's REACH program (NIH, 2018; Oregon Health } \\
\text { Authority, n.d.; Multnomah County, 2018). }\end{array}$ \\
\hline \multicolumn{2}{|c|}{ Action Area 3: Creating healthier, more equitable communities } \\
\hline $\begin{array}{l}\text { Built environment and } \\
\text { physical conditions }\end{array}$ & $\begin{array}{l}\text { Requires recognition of the causality of complexity } \\
\text { (Dubowitz et al., 2016). Just as putting a grocery store in a } \\
\text { former food desert does not automatically improve } \\
\text { community health outcomes, ADRD research participation } \\
\text { does not automatically increase if a new study is } \\
\text { announced. There are environmental and physical factors } \\
\text { affecting a person's decision to participate (Grill \& Galvin, } \\
\text { 2014; Mahon et al., 2016: Kramer, 2017). }\end{array}$ \\
\hline $\begin{array}{l}\text { Social and economic } \\
\text { development }\end{array}$ & $\begin{array}{l}\text { May require new government structures and policies } \\
\text { (Dubowitz et al., 2016). The NIH Research Capacity } \\
\text { Building, the new Director of Extramural research, the US } \\
\text { Congress earmarking } 2.8 \text { billion dollars. The NIH funding } \\
\text { for recruitment (Hudson et al., 2016; NIA, 2018; NIH, } \\
\text { 2018; Alzheimer's Association, 2019b) }\end{array}$ \\
\hline $\begin{array}{l}\text { Policy and } \\
\text { governance }\end{array}$ & $\begin{array}{l}\text { May require framing social determinant policies as a } \\
\text { complement to individual choice, rather than either/or } \\
\text { policies (Dubowitz et al., 2016). One example of this }\end{array}$ \\
\hline
\end{tabular}




\begin{tabular}{|l|l|}
\hline \multicolumn{1}{|c|}{} & $\begin{array}{l}\text { includes allowing all, some, or none of the clinical } \\
\text { research visits to occur via telemedicine or at the } \\
\text { individual's home rather than at the clinic. Engaging with } \\
\text { community members can facilitate solutions (Denhardt \& } \\
\text { Denhardt, 2003, Grill \& Galvin, 2013; Inungu et al., 2017) }\end{array}$ \\
\hline \multicolumn{1}{|c|}{ Action Area 4: Strengthening integration of health services and systems } \\
\hline Access & $\begin{array}{l}\text { Current barriers to access to healthcare include cost and } \\
\text { insurance, language and culture, transportation, office } \\
\text { hours, and historical and current mistrust of the medical } \\
\text { community (Martin et al.,. 2016). These barriers are all } \\
\text { found in the clinical research world as well, particularly } \\
\text { for the African American and ADRD communities } \\
\text { (Danner et al., 2011; Law et al., 2014; Friedman et al } \\
\text { 2015; Mahon et al., 2016) }\end{array}$ \\
\hline $\begin{array}{l}\text { Consumer experience } \\
\text { and quality }\end{array}$ & $\begin{array}{l}\text { The Triple Aim: focused goal of improving the } \\
\text { community's health and experience and lowering costs } \\
\text { (Berwick et al., 2008). The clinical research, advocacy, } \\
\text { and health sectors has a similar goal of improving the } \\
\text { experience of research participation, with the ultimate goal } \\
\text { of ADRD prevention and treatment (NIH 2018, } \\
\text { Alzheimer's Association, 2018). }\end{array}$ \\
\hline $\begin{array}{l}\text { Balance encompasses how resources are prioritized and } \\
\text { allocated between health and social services; integration } \\
\text { encompasses the meaningful connections made between } \\
\text { integration } \\
\text { the health and social sectors (Mahon et al., 2016). This } \\
\text { balance and integration can be viewed in the clinical } \\
\text { research sector as well with the prioritization of funding } \\
\text { recruitment and retention activities versus other studies/ } \\
\text { data management and retention and partnerships between } \\
\text { the research organization and the community (NIA, 2018). }\end{array}$ \\
\hline
\end{tabular}

This framework provides opportunities for people with ADRD who have not been able to use their voice, by encouraging them to define their own needs and bring solutions to the table. A core tenet of this framework is to reduce inequities among and between groups. The high diagnosis and low participation rates that African Americans face could be addressed with this framework. The Culture of Health Action Framework can serve as a map to building capacity and partnerships, recognize and mitigate barriers caused by 
structural racism, help research organizations conduct more outreach to motivate community members, and increase ADRD clinical research enrollment.

\section{Literature Synthesis and Conclusion}

The goal of finding a treatment or prevention for ADRD by 2025 is based on a variety of reasons, including: the expectation of ADRD prevalence as the older adult population drastically increases; the persistent lack of a successful prevention, treatment, or cure; and the high-costs, financially, physically, and emotionally, of living with or caring for a person with ADRD. Increasing research participation may reduce the delay or termination of studies that could potentially lead to a breakthrough. The need is persistent, as are the low participation rates; a myriad of policy, system, organization, and personal barriers stand in the way of capability and motivation to participate among people with ADRD.

Ethical guidelines and government policies that serve to fund, conduct, and disseminate clinical research protect individuals with ADRD from harm, but inadvertently strip their decision-making abilities from them. Though there is an understanding that many of the rules and structures cannot be changed, research organizations are willing to make some accommodations to meet the needs of people with ADRD. Contingency and transaction cost theories, both of which promote environmental influence while maintaining the organization's rigidity, best conceptualize how research organizations can facilitate ADRD clinical research participation. Policy theories that recognize how society, organizations, and government perceive and 
influence mobilization of people with ADRD, best illustrate how health, social, and research sector organizations can work together and with people with ADRD to promote capabilities and motivation to participate in research. Concepts of civic engagement align with suggestions and recommendations described in the clinical research literature as strategies to mitigate system, organizational, and personal barriers. The Culture of Health Action Framework can be used as a roadmap to form organization-community partnerships, facilitate motivation and empowerment, give back decision-making power to people with ADRD and promote a culture of research. In Chapter 3, an approach is presented to illustrate how the Culture of Health Action Framework will be used, in conjunction with community-engaged approaches to research, for this particular study. 


\section{CHAPTER 3: RESEARCH DESIGN AND METHODOLOGY}

\section{Chapter Organization}

This chapter offers a detailed description of the dissertation's design and methodology. It is organized in the following manner:

1. An introduction of the research question, specific aims, and the rationale.

2. An overview and rationale of community-engaged research, the approach used in this study.

3. A detailed account of the study design: two phases of semi-structured interviews designed in collaboration with a community advisory board (CAB).

4. A discussion of data collection and analytic processes.

5. Conclusion.

\section{Introduction}

The overall research question of this study asked: What factors affect clinical research enrollment among people with ADRD? The study aimed to a) identify organizational-, system-, and policy-level factors affecting ADRD clinical research enrollment, b) identify person-level factors affecting clinical research enrollment among people with ADRD, and c) develop motivational strategies and policy recommendations based on the findings from Aims 1 and 2.

As previously discussed in Chapter 2, there are several reasons why this research question was explored. ADRD is considered a public health crisis at a local, national, and 
global scale (WHO, 2017; Collins et al., 2018; Alzheimer's Association, 2019a). The U.S. Congress has allocated nearly 3 billion dollars for ADRD research to find a treatment or prevention by 2025 , and philanthropists have contributed financial resources towards the same goal (Gates, 2017; Alzheimer's Association, 2019a). The multitude of reasons why enrollment is low was explored in Chapter 2. Due to public attention and funding appropriations from Congress, the conversation on recruitment rates has expanded from the academic literature to the NIH, investigators, and funders. For example, the NIA released their national strategy to improving recruitment and diversity in ADRD research in October 2018. They briefly identified barriers to research and provided recommendations to facilitate enrollment; however, people with ADRD were not included in their working groups (NIA, 2018). The research conducted in this dissertation serves as a first step in identifying barriers and facilitators to ADRD clinical research enrollment and fills the gap in the literature by including the voices of people with ADRD.

\section{Approach}

This section provides an overview of community-engaged approaches to research. Principles of community-engaged research are identified and their application to this study is evaluated. This section ends with a rationale for why this approach was used.

\section{Overview of community-engaged research}

Community-engaged research encompasses a wide breadth of community inclusion in research and decision-making. Community engagement is defined as "the 
process of working collaboratively with and through groups of people affiliated by geographic proximity, special interest, or similar situations to address issues affecting the well-being of those people" (Centers for Disease Control and Prevention [CDC], 1997, p. 7). Various fields have contributed to the development of community engagement, including education and political science (Freire, 1968/1972; Bellah et al., 1991; Putnam, 2000; Battistoni \& Longo, 2011). In these fields, "civic" engagement is the commonly used term but is equivalent to community engagement, which is why community engagement is frequently referred to as civic engagement in Chapter 2. For this research, the term community engagement is used because it is the preferred term in the health services field.

\section{Rationale for using a community-engaged research approach}

Using community-engaged research, community members and academics collaborate and develop research and decision-making processes that best address the needs of the community (CDC, 1997). First, community-engaged research recognizes that despite a common misconception that research is objective, it is not, and it often cannot be. All individuals have different lived experiences, leading to unique perspectives (Habermas, 1963/1974). Because of these experiences and perspectives, researchers and community members have conscious and unconscious biases that can affect their research topics and processes. To establish partnerships, these unique perspectives and biases need to be identified and discussed.

Second, in community-engaged research, academics recognize and accept a) that significant power differences may exist between community participants and themselves 
and b) the effects that real or perceived power differences have on participation behavior. However, this recognition must exist before power sharing can begin. When power is shared, community empowerment, trust, and meaningful relationships develop (Michener et al., 2012).

Third, community-engaged research can enhance study rigor, relevance, and reach (Balazs \& Morello-Frosch, 2013). Rigor can be enhanced when the community directs researchers to use designs and data collection methods that best address their needs and questions. For example, scientific rigor and generalizability is enhanced with memberchecking, where community members provide feedback on the results that confirm or challenge the investigator's findings analyses (Creswell, 2000; Goldblatt, Karneili-Miller, \& Neumann, 2011). Though this technique is used often in qualitative research, it was used in this research specifically because it provided an opportunity for power-sharing by recognizing and utilizing the expertise that community members hold regarding this topic. Relevance is enhanced because the community is identifying topics and goals that matter most to them, rather than having topics and goals imposed on them; in addition, the study's process and findings are disseminated in ways that are most relevant to the community (e.g. pamphlets with clear, concise language). Reach of the findings and community impact is also enhanced through dissemination to community members, and can also lead to community testimony to policymakers, potentially resulting in policies beneficial to the community (Balazs \& Morello-Frosch, 2013).

The principles of community-engaged research set standards for how research can be community-based while allowing for flexibility to respond to community needs. The 
nine principles, as identified by Israel and her colleagues (2003) in Minkler and Wallerstein's foundational text on community-based participatory research (CBPR), are one approach to community-engaged research, and are described below:

1. CBPR recognizes the community as a unit of identity.

2. CBPR builds on strengths and resources within the community.

3. CBPR facilitates collaborative equitable partnerships in all research phases and involves an empowering and power-sharing process that attends to social inequities.

4. CBPR promotes co-learning and capacity building among all partners.

5. CBPR integrates and achieves a balance between research and action for the mutual benefit of all partners.

6. CBPR emphasizes public health problems of local relevance and ecological perspectives that recognize and attend to the multiple determinants of health and disease.

7. CBPR involves systems development through a cyclical and iterative process.

8. CBPR disseminates findings and knowledge gained to all partners and involves all partners in the dissemination process.

9. CBPR requires a long-term process and commitment to sustainability.

Studies that use a CBPR approach to research design often involve community members to a more intensive degree, as illustrated by Figure 3.1 (Minkler \& Wallerstein, 2008; Potter et al., 2010). This particular research study used a medium intensive collaboration 
style with community members, where the study is primarily researcher-designed but the community collaborates on modifications and develops dissemination strategies. The medium intensive approach to community-engaged research is used for two reasons: 1) ADRD entails progressive cognitive decline; though full partnership is infeasible, people with ADRD can and should collaborate to the extent of their abilities; and 2) a dissertation research study demonstrates that a doctoral student can conduct research independently in a time-constricted manner; thus, a long-term, community-led study is not possible. Despite their typical use for more intensive community engagement, the applicable guiding principles are reflected throughout the study design and adapted as needed. Examples are highlighted throughout this chapter.

\section{Figure 3.1 Degree of Community Engagement}

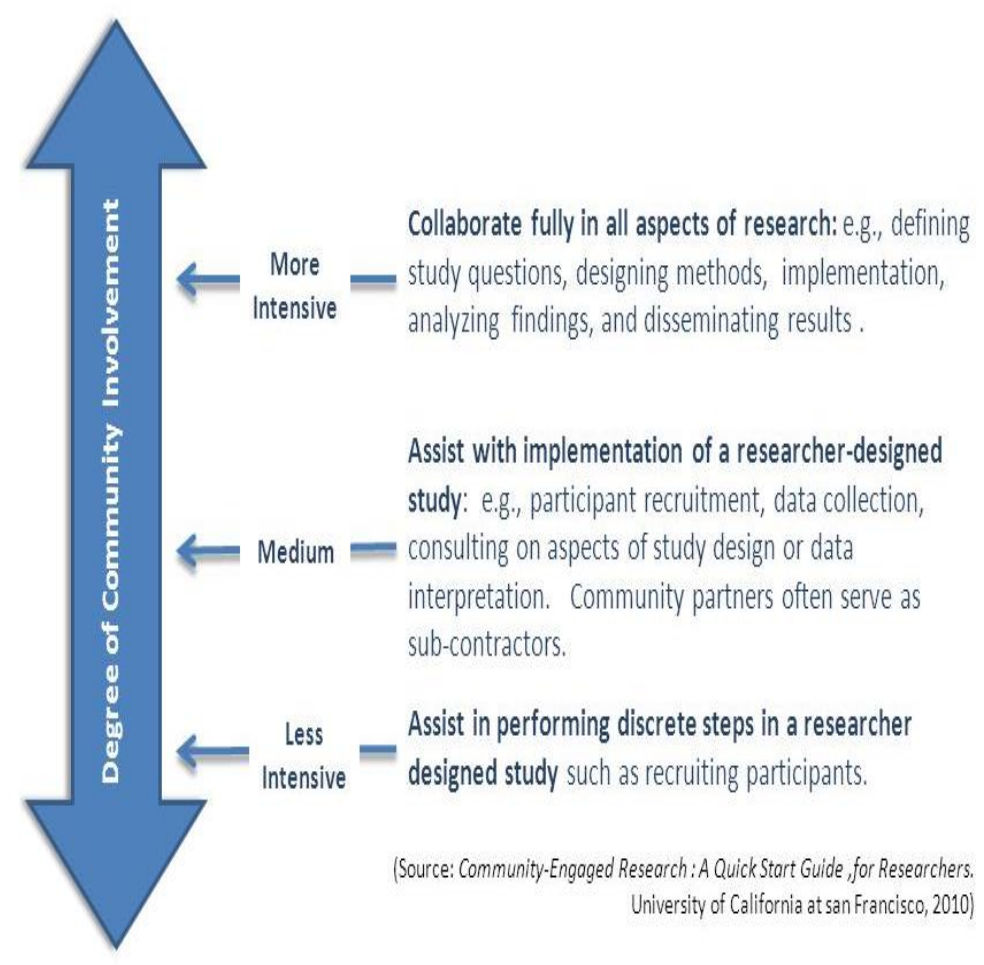




\section{African American representation}

As discussed in Chapter 2, African Americans are significantly more likely to be diagnosed with ADRD than any other race or ethnicity, yet significantly less likely to participate in clinical research for a multitude of reasons (Williams et al., 2010; Danner et al, 2011; Chin et al., 2011; Williams et al, 2011; Gilmore-Bykovskyi et al., 2019). This disparity is recognized, and the NIH has prioritized funding to reduce it and increase diversity in clinical research $(\mathrm{NIH}, 2018)$. Strategies to increase enrollment among African Americans with ADRD must derive from expertise that can only be provided by African Americans. Evidence posits that community-engaged approaches increase African American collaboration with academics (Williams et al., 2010; Huang \& Coker, 2010; Johnson et al., 2015; Harris, Pensa, Redlich, Pisani, \& Rosenthal, 2016). For these reasons, older African Americans, regardless of their cognitive status, were members of this study's CAB. In order to identify personal-level factors affecting ADRD clinical research enrollment in the African American community, the investigator attempted to mindfully sample African American dyads. Recruitment was conducted through the CAB, the OHSU Layton Aging and Alzheimer's Disease Center, which conducts clinical research that specifically focuses on the African American population, and the PreSERVE Coalition for Black/African American Memory and Brain Health, a Portlandbased "coalition of individuals from non-profits, health institutions, and the African American community that seeks to improve the health of African Americans over the age of 55" (PreSERVE, n.d.). This study originally had a recruitment goal of 50\% African Americans for the aforementioned reasons. However, the investigator was not successful 
in recruiting African Americans. The consequences of not having African Americans included in this research are discussed in Chapter 7.

\section{Conceptual Framework}

The Culture of Health Action Framework, previously discussed in Chapter 2, was chosen as a conceptual framework for several reasons. First, the framework and its action areas may be useful for articulating strategies to increase the ability and motivation to participate in ADRD clinical research. This is due to the similarities between factors affecting healthcare and factors affecting clinical research participation. Second, the voices of people with ADRD have not traditionally been included in research. This framework could be used to conceptualize an approach that encourages inclusion of people with ADRD to define their own needs and develop solutions to address structural barriers to participation. Third, the Culture of Health Action Framework has not yet been applied to the ADRD clinical research setting. Using this framework is a novel approach to organizing strategies to improve ADRD clinical research participation.

\section{Design}

The design of this study entailed two phases of semi-structured qualitative interviews. Recruitment strategies and data collection protocols were designed in collaboration with a CAB. 


\section{Use of CAB and rationale}

A CAB was established to provide formal leadership and guide study processes. CAB membership recruitment was facilitated through the Alzheimer's Association, OHSU's Layton Aging, and Alzheimer's Disease Research Center, and PreSERVE. The CAB membership was comprised of two individuals with ADRD, two current ADRD family caregivers, two ADRD advocates, two ADRD clinicians/investigators, one ADRD research assistant, and two older African American community members, as well as a dissertation committee member with experience with $\mathrm{CABs}$ and the researcher. The $\mathrm{CAB}$ was convened three times throughout the dissertation process to guide the investigator on recruitment and interview protocols and member-checking. CAB community members received a $\$ 120$ honorarium, or $\$ 30$ grocery gift card per meeting, for their contributions to the CAB. Two originally planned meetings were condensed to one meeting; community members received two $\$ 30$ grocery gift for collaborating in that meeting. These funds were reimbursed by an Oregon Partnership for Alzheimer's Research Tax Checkoff grant awarded to the researcher.

Rationale for CAB: Ian Kremer (2017), executive director of the Leaders Engaged on Alzheimer's Disease (LEAD) Coalition, suggests that CABs for dementia programs should include persons with ADRD as they have the greatest insights for developing and enhancing dementia-related services. For example, the Dementias and Neurodegenerative Research Network (DeNDRoN) in the UK includes people with dementia in their CAB, who frequently provide recruitment strategies that other members do not consider 
because they have not experienced those barriers (Iliffe, McGrath, \& Mitchell, 2013; Pickett, 2017).

CAB practices and expectations: Best practices for CABs were applied at the formative, operational, and maintenance stages (Newman et al., 2011). In the formative stage, the purpose and function of the $\mathrm{CAB}$ and membership roles must be clarified, and specific membership composition and recruitment strategies should also be developed and confirmed by the members (Newman et al., 2011). For this CAB, membership and expectations were discussed and approved at each meeting (Appendices A-D), and it was explicit that the CAB would be for a short-term project. The recruitment for this study's CAB was facilitated through the researcher's professional network of key contacts, influential people who have gained access and trust with people with ADRD and older African Americans, and snowball sampling among involved community members.

During the operations phase of the $\mathrm{CAB}$, procedures and principles, such as determination of leadership, power balance, and decision-making processes, should be reevaluated periodically and revised as necessary (Newman et al., 2011). For example, Israel's $\mathrm{CAB}$ with the Detroit urban community requires every member to get behind an idea with at least $70 \%$ of their support, whereas Nicolaidis's CAB with the autism community requires full membership consensus by showing one or two fingers on a fivefinger scale (Israel et al., 2001; Nicolaidis et al., 2011). This CAB required that all members must be "okay" with decisions before they could move forward, meaning that they did not have to "love" or "like" a decision but would be okay with it taking place. This is further described in Appendix A. At this study's CAB's first meeting, the CAB 
developed $\mathrm{CAB}$ protocols and decision-making processes based on what worked best for the community members. Accommodations for people with cognitive impairment are adaptable within the CAB. Pickett (2017) suggests individually tailoring accommodations and revising as needed given the progression of the disease. Some of the strategies from the developmental disability literature include one-on-one sessions before meetings, extra time to gather and present their thoughts, and caregiver inclusion (Nicolaidis et al., 2011; Nicolaidis et al., 2015; McDonald et al., 2016; McDonald \& Stack, 2016). Additional strategies, such as frequent breaks, using simpler language, and minute-long pauses between topic discussions for thought-processing, can be included as processes that all CAB members abide by (Lennox et al., 2005; McDonald et al., 2016; McDonald \& Stack, 2016). For this CAB, the investigator spoke individually by telephone or in-person with members who requested the meeting that the investigator offered. Frequent breaks, minute-long pauses, and simple language were also used during meetings. These strategies and processes are included in Appendices A-D.

In the maintenance phase, all partnership processes are evaluated, sustainability plans are created, and membership contributions, such as funding strategies and member stipends, are discussed and recognized (Newman et al., 2011; Nicolaidis et al., 2011; Pickett, 2017). In addition to discussing and confirming meeting expectations, each meeting ended with a five-minute debrief to evaluate and modify processes for future meetings. Because this study was a short-term project, this $\mathrm{CAB}$ will not continue after the study's completion, but communication and relationships with members will continue for dissemination projects. 
$\mathrm{A} \mathrm{CAB}$ is ultimately responsible for establishing procedures and protocols for data collection, analysis, and dissemination. The research should be reflective of active dialogue with the community. This starts with effective recruitment strategies and accommodation practices that work to ensure diverse members are able to participate. Examples are discussed in Chapter 6 and final products are included in the Appendices. Plunkett \& Chen (2016) found that many clergy, who are opinion leaders for religious communities, are unsure of dementia-related resources to which they can refer their members. They suggest developing partnerships between community organizations and churches to facilitate recruitment and develop programs to better serve people with ADRD (Plunkett \& Chen, 2016). Places of worship were not discussed by members of the $\mathrm{CAB}$ nor mentioned as a possible referral site. This limitation is discussed in Chapter 7.

\section{Two-phase qualitative interviews and rationale}

To address the first two aims of the study, presented in Figure 3.2 below, two phases of semi-structured interviews were used. Semi-structured interviews provided an opportunity to have a conversation that the interviewee could steer within the parameters of the interview protocol, establishing a more trusting relationship between the two parties (Cook, 2012). In the first stage of data collection, the investigator conducted semistructured interviews with nine clinicians, researchers, and advocates who serve people with ADRD in the Portland metropolitan area to address the first aim. These included professionals and volunteers who advocate for the ADRD community, clinicians who 
treat the ADRD community, and investigators and research assistants who conduct research with the ADRD community. The stakeholders were recruited through the researcher's professional network of contacts as well as referrals from the CAB. Interviewing nine key stakeholders provided the researcher with a breadth of perspectives until saturation was reached without hitting the threshold of researcher burden

\section{Figure 3.2 Dissertation Aims}

1. Aim 1: Identify organizational, system, and policy factors that impede or enhance clinical research enrollment among people with ADRD;

2. Aim 2: Describe personal factors that persuade or dissuade individuals with ADRD from enrolling in clinical research; and

3. Aim 3: Develop motivational strategies and policy recommendations based on the findings from Aims 1 and 2.

In Phase 2, the semi-structured interviews were conducted with 12 dyads of individuals with ADRD and their caregivers who live in or participate in research based in the Portland metropolitan area. The dyads were recruited through the CAB and key informants from Phase 1. These interviews addressed both the first and second aims (Figure 3.2). Six of the dyads currently or previously participated in ADRD clinical research. The remaining six had never participated in ADRD clinical research. Twelve dyads were chosen because this allowed the researcher to receive a breadth of differing perspectives from both those who had or had not participated in clinical research. The caregiver and person with ADRD were interviewed separately, but the person with ADRD could allow the caregiver to sit with them during the interview if they preferred as 
long as the caregiver remained quiet. Only one of twelve people with ADRD had their caregiver sit with them.

This approach was used for several reasons. First, in dyadic interviews, the relationship between two people is recognized and used as a source of information (Morgan, Ataie, Carder, \& Hoffman, 2013; Caldwell, 2014). Second, dyadic interviews are frequently used when interviewing people with cognitive impairment because it provides them with more control of the situation, reduces the need to conform to preconceived notions of what the interviewer is looking for, and empowers them to actively participate (Morgan et al., 2013; Caldwell, 2014). Dyadic interviews were intentionally conducted after the Phase 1 interviews with key informants provided context for the Portland metro area and identified insights that may not have been discussed in the literature. Phase 2 interviewees confirmed whether the findings from Phase 1 aligned with what people with ADRD and their caregivers perceived as barriers and facilitators to clinical research enrollment.

This interview process was guided by several of the CBPR principles. Through these interviews, this research recognized people living with ADRD as a unit of identity. Interviewing people with ADRD and their caregivers after key informant interviews empowered them by asking about their experiences and invited a power-sharing process by perceiving them as experts in their own field through confirmation or rejection of key informant perceptions. Recognizing their expertise further integrated and achieved a balance between research and action by providing research organizations, advocates, and health clinics with recommendations to promote changes that increase research 
participation, mutually benefitting both groups. Further, these semi-structured interviews, drafted in collaboration with the $\mathrm{CAB}$, were designed in a way that offered the interviewee the ability to take the conversation in a direction that allowed them to discuss their most pressing issues and concerns and provided the opportunity to articulate factors affecting their decision to participate in clinical research.

\section{Data Collection}

The two-phase approach to semi-structured qualitative interviewing was utilized to address the first two aims of the study (Figure 3.2) while also confirming if the interviewed people with ADRD and their caregivers' perceptions of barriers and facilitators to clinical research enrollment aligned with those of ADRD researchers, clinicians, and advocates. In this section, each phase of data collection is discussed, including the rationale, recruitment and interview protocols, the CAB's involvement in data collection, and IRB processes.

\section{Phase 1 interviews with key informants}

In the first stage of data collection, the investigator conducted semi-structured interviews with key informants who serve people with ADRD in the Portland metropolitan area to address the first aim. Key informants included professionals and volunteers who advocate for, clinicians who treat, and investigators and research assistants who conduct research for or with people with ADRD. 
Recruitment approach and development of protocols: The investigator established relationships with the PSU Institute on Aging, the Alzheimer's Association, the Layton Aging and Alzheimer's Disease Center (OHSU), and PreSERVE. The investigator emailed clinicians, researchers, and advocates who were suggested as good key informants by members of the aforementioned organizations. Nine key informants were selected based on the organization they worked for, to ensure that there was diversity in organizational representation in this study. These key informants were not paid.

Interview protocols: Table 3.1 presents an overview of the domains that were addressed and the questions that were asked during Phase 1 and approved by the CAB. Key informants were asked about their experience of working with people with ADRD, their knowledge of clinical research, if and how they receive information about clinical research opportunities, dissemination of those opportunities, their perceptions of organizational-, system-, and policy-level factors affecting clinical research recruitment and retention, and suggestions for improving ADRD clinical research enrollment. The questions were derived from various frameworks and literature described in Chapter 2. The interviews were audio-recorded, transcribed and deductively and inductively coded for themes that addressed the first and second study aims.

\section{Table 3.1 Phase 1 Interview Domains}

\begin{tabular}{|l|l|l|}
\hline Domains & Questions & Derives from \\
\hline Engagement \\
with people with & Describe this organization and your & Culture of Health $(\mathrm{CoH})$ \\
ADRD & role within it. & Action Area 1 \\
& How does your organization engage & Civic engagement literature \\
& people with ADRD? & $\begin{array}{l}\text { Policy feedback theory } \\
\text { Social construction theory }\end{array}$ \\
\hline
\end{tabular}




\begin{tabular}{|c|c|c|}
\hline & & $\begin{array}{l}\text { Clinical research and } \\
\text { community engagement } \\
\text { literature }\end{array}$ \\
\hline $\begin{array}{l}\text { Policies and } \\
\text { funding } \\
\text { mechanisms }\end{array}$ & $\begin{array}{l}\text { What policies or funding } \\
\text { mechanisms enhance your work? } \\
\text { What policies or funding } \\
\text { mechanisms have made it easier or } \\
\text { more difficult to engage with } \\
\text { people with ADRD and/or increase } \\
\text { knowledge of opportunities? }\end{array}$ & $\begin{array}{l}\text { CoH Action Areas } 2 \text { and } 3 \\
\text { Policy Feedback theory } \\
\text { Contingency theory } \\
\text { Clinical research barriers } \\
\text { and facilitators literature }\end{array}$ \\
\hline Partnerships & $\begin{array}{l}\text { What partnerships are you involved } \\
\text { in that relate to people with ADRD? } \\
\text { How is this partnership funded? } \\
\text { How are stakeholders involved? }\end{array}$ & $\begin{array}{l}\text { CoH Action Area } 2 \\
\text { Clinical research and } \\
\text { community engagement } \\
\text { literature } \\
\text { Civic engagement literature } \\
\text { Contingency theory }\end{array}$ \\
\hline $\begin{array}{l}\text { Clinical } \\
\text { research } \\
\text { (values) }\end{array}$ & $\begin{array}{l}\text { How valued is clinical research at } \\
\text { this organization? } \\
\text { Describe your process for informing } \\
\text { people with ADRD and their } \\
\text { caregivers of research opportunities } \\
\text { [or recruiting and enrolling if } \\
\text { organization is a research } \\
\text { organization]. How has that process } \\
\text { changed since it was implemented? }\end{array}$ & $\begin{array}{l}\mathrm{CoH} \text { Action Areas } 1 \text { and } 4 \\
\text { Policy feedback theory } \\
\text { Contingency theory } \\
\text { Civic engagement literature } \\
\text { Transaction cost theory } \\
\text { Clinical research barriers } \\
\text { and facilitators literature }\end{array}$ \\
\hline $\begin{array}{l}\text { Clinical } \\
\text { research } \\
\text { (perceptions) }\end{array}$ & $\begin{array}{l}\text { What are some factors that you } \\
\text { think may facilitate recruitment? } \\
\text { What about factors that impede } \\
\text { recruitment? } \\
\text { What are your recommendations for } \\
\text { increasing ADRD clinical research } \\
\text { participation? }\end{array}$ & $\begin{array}{l}\text { CoH Action Areas } 3 \text { and } 4 \\
\text { Policy feedback theory } \\
\text { Transaction cost theory } \\
\text { Social construction theory } \\
\text { Critical race theory } \\
\text { Clinical research barriers } \\
\text { and facilitators literature } \\
\text { Clinical research and civic } \\
\text { engagement literature } \\
\text { ADRD specific barriers and } \\
\text { facilitators literature }\end{array}$ \\
\hline
\end{tabular}

CAB involvement in Phase 1: The investigator created a draft of recruitment and interview protocols based on best practices from the academic and grey literature, and shared these with $\mathrm{CAB}$ members before their first meeting. At the meeting, $\mathrm{CAB}$ 
members were asked to draw upon their expertise to suggest changes; recruitment and interview protocols were modified based on their suggestions.

\section{Phase 2 dyadic interviews with people with ADRD and their caregivers}

The second stage of data collection also consisted of semi-structured interviews but included a sample of 12 dyads of individuals with ADRD and caregivers living in the Portland metropolitan area $(n=24)$. These interviews addressed both the first and second aims. To ensure the data collected articulated the personal reasons for why people with ADRD members both have and have not participated in clinical research, six of the dyads had participated in ADRD clinical research while the remaining six had never participated in ADRD clinical research.

Recruitment approach and protocols: At the end of each Phase 1 interview, key informants were asked if they were willing to leave a recruitment flyer in their office and/or to send a recruitment flyer to their mailing list and people with ADRD and their caregivers who they believed would be interested in participating in the study. Through CAB referrals, dyads were also recruited through the Memory Café hosted by Multnomah County (a social group for people with ADRD and caregivers) and an Alzheimer's Association early-stage memory loss support group. An initial telephone screening was conducted with one member of the dyad to determine eligibility based upon the following factors: a) if they both fluently spoke English, could engage in a conversation, and discussed their perceived values; b) if the person with MCI or ADRD had a clinician-assessed diagnosis; and c) if one member of the dyad had ADRD, if they were diagnosed as early or moderate stage within the last six months (see Appendix G). 
Although MCI is not considered a form of ADRD, clinical research studies frequently include people with MCI given hypotheses about the progression of ADRD, and that people with $\mathrm{MCI}$ are significantly more likely to progress to dementia (Roberts and Knopman, 2013; Rabinovici et al., 2019). Each interviewee received a \$25 gift card to the grocery store of their choice as compensation for their time.

It is important to note that some of the recruitment strategies influenced dissemination of local dementia research opportunities. In one case, the researcher was able to attend an Alzheimer's Association early memory loss support group for caregivers and people with dementia to recruit for this study. The researcher spent a few minutes describing the study and shared recruitment flyers. The researcher found this to be a useful resource, and an opportunity for researchers to request to speak to support groups is listed on the Alzheimer's Association's webpage, so the researcher encouraged other researchers to contact the staff member and ask to present at the support groups. Shortly thereafter, the researcher was notified by the program director that support group facilitators had received a large number of requests and asked that the researcher no longer promote this resource as this was not the purpose of the support groups, and instead to share TrialMatch with fellow researchers as a resource for promoting their studies.

Conversely, after recruiting for this study at a county-sponsored Memory Café in August 2019, the program coordinator of the Memory Café advised the researcher that her employer could choose to help sponsor the café and share study opportunities with participants, who are people with dementia and their caregivers. The OHSU Layton 
Center began a partnership with Multnomah County's Memory Café in September 2019. In addition to offering a venue for sharing resources about research participation opportunities, the sponsorship requires a representative to help set up, clean up and converse with Memory Café attendees and sponsors. This requirement has resulted in relationships among community members, community organizations and research staff. Further, it has led to several initial contacts between OHSU research staff and potential participants. Although there is no evidence as yet indicating how many participants have been recruited due to this new partnership, this could be explored in the future.

Interview protocols: Table 3.2 illustrates the domains and interview questions for Phase 2. Dyads were asked about their first signs of ADRD or MCI, their knowledge of clinical research, how and if they receive information about opportunities, their perceptions of clinical research, their decision to enroll or not enroll in studies, and suggestions for improving ADRD clinical research enrollment. Questions derived from the various frameworks and literature described in Chapter 2. 
Table 3.2: Phase 2 Interview Domains

\begin{tabular}{|c|c|c|}
\hline Domain & Questions & Derives from \\
\hline $\begin{array}{l}\text { ADRD } \\
\text { diagnosis }\end{array}$ & $\begin{array}{l}\text { When did you first notice signs of } \\
\text { MCI/dementia? } \\
\text { When did you receive a diagnosis? }\end{array}$ & $\begin{array}{l}\text { CoH Action Area } 4 \\
\text { Clinical research barriers and } \\
\text { facilitators literature } \\
\text { Policy feedback theory }\end{array}$ \\
\hline $\begin{array}{l}\text { Motivations } \\
\text { to } \\
\text { participate } \\
\text { in research }\end{array}$ & $\begin{array}{l}\text { Please describe in your own words } \\
\text { what "clinical research" is. } \\
\text { What do you think about clinical } \\
\text { research? } \\
\text { What made you decide to } \\
\text { participate/not participate? } \\
\text { If you have participated, what was } \\
\text { your experience like? Would you } \\
\text { participate again (or participate in } \\
\text { another study)? }\end{array}$ & $\begin{array}{l}\text { CoH Action Areas } 1,3 \text {, and } 4 \\
\text { Policy feedback theory } \\
\text { Clinical research barriers and } \\
\text { facilitators literature } \\
\text { Social construction theory } \\
\text { Transaction cost theory } \\
\text { Civic engagement literature } \\
\text { Critical race theory }\end{array}$ \\
\hline $\begin{array}{l}\text { Capacity to } \\
\text { participate } \\
\text { in research }\end{array}$ & $\begin{array}{l}\text { Have you heard of clinical research } \\
\text { opportunities? What about dementia } \\
\text { research? } \\
\text { Are there any barriers to make it more } \\
\text { difficult for you to participate? What } \\
\text { about things that make it easier? } \\
\text { What makes you not want to } \\
\text { participate? } \\
\text { If you could tell research organizations } \\
\text { anything to improve research } \\
\text { participation, what would it be? }\end{array}$ & $\begin{array}{l}\text { CoH Action Areas } 1,3 \text {, and } 4 \\
\text { Policy feedback theory } \\
\text { Transaction cost theory } \\
\text { Contingency theory } \\
\text { Clinical research barriers and } \\
\text { facilitators literature }\end{array}$ \\
\hline $\begin{array}{l}\text { Agreement } \\
\text { with Aim } 1 \\
\text { findings }\end{array}$ & $\begin{array}{l}\text { These are some of the } \\
\text { facilitators/barriers from research } \\
\text { organizations, clinicians, and } \\
\text { advocates. Please tell me if you agree } \\
\text { or disagree and why. }\end{array}$ & Aim 1 findings \\
\hline
\end{tabular}

Questions were semi-structured and based on the literature, policies, findings from the key informant interviews, and the CAB. The interviews were audio-recorded, transcribed and inductively coded for themes that address the first and second aims. 
CAB involvement: The investigator created a draft of recruitment and interview protocols based on best practices from the academic and grey literature as well as suggestions from the Phase 1interviewees. These drafts were submitted to CAB members before their second meeting. At the meeting, $\mathrm{CAB}$ members provided their expertise and suggest changes. Afterward, the protocols were modified based on their suggestions.

\section{IRB Processes}

The study processes were conducted per PSU's IRB rules and regulations.

\section{IRB process for semi-structured interviews}

To ensure informed consent and confidentiality, before the start of the interviews, written informed consent by all interviewees was obtained per PSU IRB requirements. Informed consent provided the purpose of the interviews and let the interviewees know that they would be recorded but their information would be kept confidential and will not be traced back to them.

Interviewees were given a six-digit alphanumeric code number and each interview was given a four-digit alphanumeric code. The investigator took handwritten field notes during the interviews. Post-interview field notes were either handwritten or audiorecorded and transcribed after the interview. Post-interview, the hard copy was scanned, saved to Google Drive, a secure and password protected file storage site, and immediately shredded. All voice recorded field notes and interviews were transcribed as soon as possible using Rev.com and stripped of all identifiers. The field notes, 
transcriptions of interviews and the master file containing the alphanumeric coding information were password protected on the investigator's secure laptop.

\section{Process for IRB approval}

To prepare for PSU's IRB approval, the investigator completed Collaborative Institutional Training Initiative (CITI) human subjects research training as per PSU requirements. After a successful dissertation proposal defense, the IRB application was submitted for review and approval (PSU IRB\# 184814). The application included the IRB application and all relevant supporting documents, including recruitment materials for key informant and dyadic interviews (Appendices E and F), informed consent for the key informants, ADRD caregiver, and person with ADRD (Appendices H-J), and the interview protocols for the two phases of interviews (Appendices K-M). Interviews did not take place until the IRB application was approved. A list of factors based upon Phase 1 findings (Appendix $\mathrm{N}$ ) was submitted as a modification and approved by the IRB before Phase 2 interviews took place. As previously mentioned, the investigator did not need to obtain IRB approval to recruit and convene the CAB. Modifications to the recruitment, informed consent, and interview protocols as a result of $\mathrm{CAB}$ review were submitted for final approval by the IRB before they were administered.

\section{Analytic Processes}

Analysis of semi-structured interviews is, by nature, an iterative process where analysis is intertwined with data collection. Johnson and Rowlands (2014) explain the process well:

"After several interviews, however, the interviewer begins to build a stock of knowledge about the research questions and, in most cases, feeds some of this 
information back to the informants in subsequent interviews, after those same questions have been covered...as the research develops, the interviewer should keep and review their own jottings and notes and review prior interviews when possible, or when transcripts become available...the later interviews of an indepth interviewing project are usually more focused on specific probes and verification of what has been learned in the earlier interviews" (p. 14).

The investigator took field notes during and after each interview and analyzed them immediately post-interview. The approach to research, coding, and analysis is further described below.

\section{Approach to the research}

A phenomenological approach was used for this study. In phenomenology, data is collected from people who have first-hand knowledge, or a lived experience, of a phenomenon in order to develop a better understanding of it (Creswell, 2013). In this study, the phenomenon is ADRD clinical research participation.

\section{Coding and analysis}

The codebook emerged from the interviews, but codes were informed by the investigator's a priori knowledge of the literature and various theories and framework, including the Culture of Health. Codes included sentences or sentence fragments that represented data and concepts (Miles, Huberman, and Saldana, 2020).

The study was informed by thematic analysis. In thematic analysis, deductive and inductive approaches can be used to identify themes from the data. (Lapadat, 2010). Most of the analysis was deductive, meaning the codes were analyzed with the notion that they may align with orienting concepts derived from the literature and theories (Miles et al., 2020). An inductive approach was also used to code and analyze themes that did not correspond with existing literature and theories. This was done so themes could emerge 
from the data organically and mitigate the possibility of coding those themes based on current literature rather than the interviews (Lapadat, 2010). The coding process began by coding broad themes then coding with details. For example, if an interviewee noted they were unaware of ADRD clinical research opportunities, it was coded as "ability, barrier, awareness." Conversely, if an interviewee described a policy that led to funding partnerships, it was coded as "facilitator, ability, then policy, funding, partnership."

The focus of the analysis was to identify factors affecting ADRD clinical research participation. Identified factors were compared and contrasted among interviewees and with organizational theories and frameworks to identify patterns, commonalities, and outliers. This led to several major themes and subthemes that are discussed in Chapters 4 and 5. After identifying themes and subthemes, recommendations and strategies were developed and organized using the Culture of Health Action Framework, and are discussed in Chapter 6.

\section{CAB involvement}

After analysis of transcripts from each interview phase, the findings were provided to the $\mathrm{CAB}$. The $\mathrm{CAB}$ engaged in member-checking and interpretation, where community members reviewed the analysis to confirm or reject investigator takeaways. Summaries of findings were sent to the $\mathrm{CAB}$ one week before each of the second and third meetings. During the meetings, the investigator used 3M Easel pad Post-it notes with the findings written on them and adhered them to the wall. Members were asked to write their initials on color-coded Post-it notes and to add them to the findings that they felt were important, surprising, or required more detail. They were also asked to note if 
they felt anything was missing. After this activity, each finding was discussed, with the information on the Post-its used to facilitate conversation.

\section{Conclusion}

The literature posits that policy, system, organizational, and personal factors play a role in people with ADRD's capacity and motivation to participate in clinical research. The literature also suggests that the expertise of people living with ADRD must be incorporated to identify factors and develop strategies to improve ADRD clinical research enrollment. This study was designed in such a way to identify these factors through insights of researchers, clinicians, advocates, people with ADRD and their caregivers, and utilizes a community-engaged approach to enhance the rigor, relevance, and reach of the research. Chapters 4 and 5 discuss and synthesize the findings from the two phases of interviews. Chapters 6 introduces recommendations to improve ADRD clinical research participation using the Culture of Health Action Framework to conceptualize and organize them. Lastly, Chapter 7 presents conclusions, limitations, and implications for future research. 


\section{CHAPTER 4: ORGANIZATIONAL, SYSTEM, AND POLICY FACTORS AFFECTING DEMENTIA CLINICAL RESEARCH PARTICIPATION}

\section{Introduction}

Alzheimer's disease and related dementias (ADRD) affect nearly seven million people in the United States, and rank in the top ten of causes of death and the most expensive diseases to treat, both in actual costs for treatment and care and caregiving burden (Alzheimer's Association, 2019a). ADRD clinical trials have a failure rate of over 99\%, which is due to a lack of evidence of efficacy as well as slow or failed participant recruitment (Cummings, Morstorf \& Zhong, 2014; Cummings, Lee, Morstorf, Ritter, \& Zhong, 2017). Numerous pharmaceutical companies (Axovant, Eli Lilly, Eisai, and Merck) halted their trials in the past few years after multiple study failures (Axovant, 2018; Merck \& Co; 2018; AstraZeneca; 2018). Pfizer terminated their ADRD research arm in 2018 (DeStrooper, 2018; Hawkes, 2018).

With no current prevention, treatment, or cure and an anticipated prevalence of over 18 million by 2050 in the United States alone, the need to find an effective intervention is urgent (Alzheimer's Association, 2019a). An essential element in finding an intervention is ensuring that there are enough participants in clinical research studies to determine if proposed interventions are feasible and efficacious. ADRD clinical research studies have difficulty recruiting and enrolling participants. Nearly threequarters of people with ADRD are ineligible to participate in ADRD research (Grill \& Galvin, 2014). Additionally, there are a variety of organizational, system level, and 
policy level factors, both general and ADRD-specific, affecting motivation and ability to join a study (Grill \& Galvin, 2014; NIA, 2018). These factors, with relevant citations from the literature, are introduced in Table 4.1 and are described below.

Table 4.1: Factors Affecting ADRD Research Participation

\begin{tabular}{|l|l|l|}
\hline \multicolumn{1}{|c|}{ Organizational factors } & \multicolumn{1}{|c|}{ System level factors } & \multicolumn{1}{|c|}{ Policy level factors } \\
\hline $\begin{array}{l}\text { Facilitators or barriers that } \\
\text { occur within an } \\
\text { organization, or between an } \\
\text { organization and its } \\
\text { sponsor or funder }\end{array}$ & $\begin{array}{l}\text { Facilitators or barriers that } \\
\text { span two or more } \\
\text { organizations }\end{array}$ & $\begin{array}{l}\text { Facilitators or barriers that } \\
\text { affect organizational or } \\
\text { system rules, processes, or } \\
\text { activities due to created or } \\
\text { enacted policies }\end{array}$ \\
\hline $\begin{array}{l}\text { Data and compliance } \\
\text { oversight (Califf, 2006; } \\
\text { Kramer, Smith, \& Califf, } \\
\text { 2012; Hemminki, 2016) }\end{array}$ & $\begin{array}{l}\text { Communication about } \\
\text { research opportunities } \\
\text { (Mahon et al., 2016; } \\
\text { Anderson et al., 2018) }\end{array}$ & $\begin{array}{l}\text { Data and compliance } \\
\text { oversight (Califf, 2006; } \\
\text { Kramer, Smith, \& Califf, } \\
\text { 2012; Hemminki, 2016) }\end{array}$ \\
\hline $\begin{array}{l}\text { Eligibility criteria (Rollin- } \\
\text { Sillaire et al., 2013; Grill \& } \\
\text { Galvin, 2014; Adams, } \\
\text { Caffrey, \& McKevitt, } \\
\text { 2015) }\end{array}$ & interest (Meador, 2015) & $\begin{array}{l}\text { IRB/Security protocols } \\
\text { (Hudson et al, 2016) }\end{array}$ \\
\hline $\begin{array}{l}\text { Recruitment strategies } \\
\text { (Sood et al., 2009; Law et } \\
\text { al., 2014; Mahon et al., } \\
\text { 2016; \& Galvin, 2014; }\end{array}$ & $\begin{array}{l}\text { Community engagement } \\
\text { (Grill \& Galvin, 2014; } \\
\text { Friedman et al., 2015, } \\
\text { Mahon et al., 2016) }\end{array}$ & $\begin{array}{l}\text { Recruitment and diversity } \\
\text { policies (King 2007; } \\
\text { Huang \& Coker, 2010; }\end{array}$ \\
\hline $\begin{array}{l}\text { Relationships with people } \\
\text { with ADRD/caregivers } \\
\text { (Williams, Meisel, } \\
\text { Williams, \& Morris, 2011) }\end{array}$ & $\begin{array}{l}\text { Structural elements (Grill } \\
\text { \& Galvin, 2014; Mahon et } \\
\text { al., 2016) }\end{array}$ & $\begin{array}{l}\text { Dissemination policies } \\
\text { (Israel et al., 2008; Carlisle } \\
\text { et al., 2015; Mahon et al., } \\
\text { 2016) }\end{array}$ \\
\hline $\begin{array}{l}\text { IRB/Security protocols } \\
\text { (Hudson et al., 2016) }\end{array}$ & & $\begin{array}{l}\text { Funding opportunities } \\
\text { (NIH, 2018) }\end{array}$ \\
\hline $\begin{array}{l}\text { Community engagement } \\
\text { (Grill \& Galvin, 2014; } \\
\text { Mahon et al., 2014) }\end{array}$ & & \\
\hline
\end{tabular}

For the purposes of this study, organization level factors are defined as facilitators or barriers that occur within an organization, or between an organization and its sponsor 
or funder, such as a research organization and its study sponsor. System level factors are facilitators or barriers that span two or more organizations, such as those involving a research organization and a healthcare organization. Policy level factors are facilitators or barriers that affect organizational or system rules, processes, or activities due to created or enacted policies. In some cases, factors may exist at multiple levels. As Table 4.1 illustrates, the aggregation of organizational, system, and policy factors contributes to the low participation rates in ADRD clinical research. Several organizational and policy theories can be used to conceptualize how these factors affect ADRD research participation.

\section{Organizational factors}

From an organization level perspective, research and health organizations are considered open rational systems; they have structures and processes that they must adhere to, but can adapt to environmental needs (Scott, 1987). Contingency theory can be applied to better understand the structure of these organizations. Contingency theory posits that the best way to structure an organization is contingent on both the needs of the organization and its environment while recognizing some structures cannot be changed (Lawrence \& Lorsch, 1957; Mintzberg, 1983). For example, national and organizationwide data collection and monitoring, while necessary to ensure ethical and clinical regulatory compliance, account for a large proportion of study resources, minimizing staff time and funding allocated to participant recruitment and retention (Califf, 2006; Kramer, Smith, \& Califf, 2012; Hemminki, 2016). 
Although research organizations are unable to modify these requirements, they can adjust other processes to meet the needs of people with ADRD. For example, study partners are required to participate with the individual with ADRD to ensure protocol adherence and appointment attendance, ensuring further difficulty due to the requirement that study coordinators need to recruit twice the number of people (Karlawish et al., 2008; Watson et al., 2014; Grill \& Galvin, 2014; Cary et al., 2015; Black et al., 2017). Although this is something that cannot (and should not) be changed, modifications could be made to increase accessibility and ease of participation for caregivers, such as conducting interviews over the phone or offering study visits outside of traditional office hours to fit the caregiver's schedule. When using contingency theory to explain these processes, organizations recognize that data compliance and monitoring and the need for study partners cannot be adjusted, other processes can be modified to better fit environmental or community/individual needs.

Often, recruitment processes are developed and implemented at the organizational level, or between a research team and their study sponsors. While the NIH requires their funded studies to include underrepresented groups, biopharmaceutical companies, which sponsor over $65 \%$ of Alzheimer's disease clinical trials, do not have these requirements (Cummings, Lee, Mortsdorf, Ritter, \& Zhong, 2017). Studies conducted in the United States and the European Union found that study eligibility criteria can severely restrict the enrollee pool of a research study, making it difficult to obtain an adequate number of participants within the proposed time frame (Kramer, 2012; Adams et al. 2015; Mahon et al., 2016). Although research protocols aim to protect human participant safety, there are 
cases of private study sponsors requiring unsubstantiated ineligibility criteria, such as comorbidities or medications, to avoid risk or delay of FDA approval (Kramer et al., 2012). Study organizations and sponsors may also impose penalties for not recruiting at expected levels, resulting in avoidance of underrepresented groups in research,

particularly African Americans (King et al., 2007; Uybico, Pavel, \& Gross, 2007; Huang \& Coker, 2010). This is especially damaging for ADRD studies as African Americans are twice as likely to develop ADRD and more likely to be diagnosed in the later stages of ADRD (Chin, Negash, \& Hamilton, 2011; Danner, Darnell, \& McGuire, 2011; Bonds \& Lyons, 2018). If research organizations work with study sponsors to modify their recruitment processes and unsubstantiated eligibility criteria and enact policies to include underrepresented groups, they can address environmental needs and facilitate ADRD clinical research participation.

\section{System level factors}

Contingency theory can also be used to examine system level factors. Community engagement among research, health, and social sectors can facilitate clinical research recruitment and retention (Frew et al., 2011; Grill \& Galvin 2014; Watson et al., 2014; Mahon et al., 2016; Inungu, Bender, \& Geiber, 2017). Community-engaged strategies result in the research organization's developing knowledge of target populations' risk behaviors, which are essential to inform effective prevention strategies as they can both affect and inform research design, recruitment, and retention (Voytek et al., 2011; Luzi et al., 2011; Inungu et al., 2017). When research organizations partner with local health providers and social groups, they promote trust between the community and themselves 
(Gifford et al., 2002; Frew et al., 2011; Mahon et al., 2016; Inungu et al., 2017).

Recognizing and reducing other structural barriers, such as transportation and geographic distance, also facilitates community member buy-in and subsequent participation, (Giffords 2002; Frew et al., 2011; Burke, 2014; Mahon et al., 2016, Inungu et al., 2017;

Croff et al., in press). Working with the community leads to greater knowledge of community needs, and organizational structures and processes can be modified to address those needs.

Transaction cost theory can be used to examine system level factors affecting ADRD research participation. In transaction cost theory, every transaction between two parties is subject to a cost; if the perceived cost is too high for either party, the transaction will not happen (Williamson, 1975). Hemminki (2016) argues that the time spent auditing and checking procedural formalities does not make research more ethical, and, in fact, the rules, procedures, and costs associated with clinical research may deter clinicians and organizations from conducting research. Many individuals assume that their providers will inform them of studies (Anderson et al., 2018), but clinicians may not view advocating for research participation as a necessary piece of their clinical practice or may view it as an unnecessary utilization of limited time, money, and workforce (Sugarman, Getz, Speckman, Byrne, \& Emanuel, 2005; European Science Foundation, 2011; Kramer et al., 2012). In these cases, the transaction costs for clinicians to learn and share information about clinical trials may be too high for providers, which negatively affects people who seek their providers' recommendations for studies. 


\section{Policy level factors}

Social construction posits that policies are based on societal perceptions of groups of people (Schneider, Ingram, \& de Leon, 2014). Changes in a group's political power and social construction result in changes in public and personal perceptions, leading to policy change and further changes in the target population's material and interpretive effects, political power, and social construction (Schneider et al., 2014). Given the low recruitment rates for ADRD clinical research, interest has increased in researching and evaluating effective strategies to recruit specifically for ADRD research. The U.S. Congress allocated 2.3 billion dollars for ADRD research in 2019 with the hope of eradicating this set of diseases by 2025 , and Bill Gates has invested $\$ 50$ million into his Dementia Discovery Fund with a specific focus on participant enrollment (Hodes, 2018; Gates, 2017).

Social construction is incorporated within another theory, policy feedback theory (PFT). PFT recognizes that payments, goods, rules and procedures and policies affect a person's predisposition and capabilities to participate in society (Skocpol, 1992), or in this case, ADRD clinical research. The NIH has taken steps to reduce bureaucratic practices, such as permitting a single IRB review and approval for multi-site studies, yet $\mathrm{NIH}$ directors acknowledge that more work needs to be done to further reduce systemic and bureaucratic barriers to enhance and advance clinical research (Hudson, Lauer, \& Collins, 2016; NIH 2016).

Disseminating results directly to those involved in research is frequently recommended in the literature (Israel et al., 2008; Carlisle et al., 2015; Mahon et al., 
2016), yet NIH policies only require NIH-funded studies or studies of FDA-approved drugs or devices to disseminate their results of clinical trials via PubMed Central and ClinicalTrials.gov (Hudson et al., 2016; NIH, 2016b). However, these results are not written in lay language, and articles are often hidden behind a paywall, resulting in them being inaccessible to those without scientific literacy, fiscal means to purchase articles, or awareness of how to access articles for free. Further, researchers are still not required to directly update participants with the results of the study in which they participated. Thus, these dissemination policies are geared more towards the scientific community than study participants.

Beginning in 2016, the National Institute on Aging (NIA) convened working groups to identify strategies to improve recruitment and retention in ADRD studies. Their report was released in 2018; it emphasizes the concept of local community collaboration, with four key strategies: (a) increase awareness and engagement; (b) build and improve research infrastructure; (c) engage local communities and support participants; and (d) develop an applied science of recruitment (NIA, 2018). The aforementioned factors affecting ADRD clinical research, and the literature in general, do not incorporate concepts of local community collaboration. Thus, research is needed to contextualize how policy, system, and organizational factors affect local ADRD research participation.

\section{Purpose of this study}

This study aimed to identify organizational, system, and policy level factors affecting ADRD clinical research participation in the Portland, OR metropolitan area. Roughly 67,000 Oregonians over the age of 65 are currently diagnosed with Alzheimer's 
disease, and this number is expected to increase to 84,000 by 2025 without an intervention to halt its increase (Alzheimer's Association, 2019a). This number represents about $9 \%$ of adults 65 and older living in Oregon, slightly less than the national average of 10\% (U.S. Census Bureau, 2018; Alzheimer's Association, 2019a). However, the actual numbers of those living with ADRD are higher because the figures cited do not include people under the age of 65 or with related dementias. Additionally, the number does not include individuals who exhibit symptoms but are un- or misdiagnosed, which could be as high as 60\% (Lang et al., 2017) Although the research cited above describes these factors, it focuses on general, national factors. Although these factors certainly affect local ADRD research efforts, they do not speak to specific state or local factors and the effects of local efforts.

\section{Methodology}

This paper describes organizational, system and policy factors affecting ADRD clinical research participation that were identified by ADRD clinicians, researchers, and advocates interviewed for this study. The larger dissertation project further identified factors affecting the decision of a person with ADRD to participate in clinical research. In addition to identifying organizational, system, and policy level factors affecting participation in ADRD research studies, the dissertation also sought to identify personal factors affecting ADRD participation as well as provide recommendations to healthcare providers, research organizations, policymakers, and advocacy groups. 


\section{Study design}

To assess organizational, system and policy factors affecting ADRD clinical research, the investigator worked with several local health providers, researchers, community organizations, and a community advisory board (CAB) to identify ADRD clinicians, researchers, and advocates to offer expert opinions on factors affecting ADRD clinical research participation. Semi-structured interview questions were derived from the literature and various theoretical frameworks. Interviewees were ultimately selected based on the organization they worked for to ensure diversity in organizational representation. Potential clinicians, researchers, and advocates were recruited by email or phone. Interviewees were not paid. All protocols were reviewed and approved by the Portland State University Institutional Review Board (IRB \#184914).

The investigator adopted a community-engaged approach to this research by identifying interviewees based on community referrals, as well as through the development and involvement of a community advisory board (CAB). The investigator chose to use a community-engaged approach to enhance study rigor, relevance, and reach, as well as for its strength in identifying community-specific factors and strategies (Balasz \& Morello-Frosch, 2013; NIA, 2018).

$\mathrm{A} C \mathrm{CAB}$ was established to advise and guide study processes. $\mathrm{CAB}$ recruitment was facilitated through the NIA-Layton Aging and Alzheimer's Disease Center (NIALayton Center), the Alzheimer's Association, and the PreSERVE Coalition for Black/African American Memory and Brain Health. CAB members included researchers, clinicians, advocates, individuals with ADRD, current and former caregivers, older 
African Americans, and a member of the researcher's dissertation committee who has experience working with $\mathrm{CABs}$ in community-based research. The 12-member $\mathrm{CAB}$ met three times during the study to guide the investigator on recruitment and interview protocols, interpret findings, and develop dissemination strategies. CAB community members were given a grocery gift card for each meeting they attended or contributed to, in recognition of their contributions to the $\mathrm{CAB}$.

\section{Data collection and analysis}

Semi-structured interviews with clinicians, advocates, and researchers were completed between April 2019 and June 2019. Interviewees were asked about their experience of working with people with ADRD; their knowledge of clinical research and related policies; if and how they receive information about clinical research opportunities and dissemination of those opportunities; their perceptions of organizational, system, and policy level factors affecting clinical research recruitment and retention; and suggestions for improving ADRD clinical research enrollment. Interviews were audio-recorded, and the investigator took field notes during and after each interview. Interviews were deidentified and transcribed using transcription services (Rev.com), then uploaded to Atlas.ti 8.1 software for coding and thematic analysis (Scientific Software Development GmbH, Berlin, Germany).

The codebook emerged from the interviews, but codes were informed by the investigator's a priori knowledge of the literature and various theories and framework, including the Culture of Health (Robert Wood Johnson Foundation, 2016). Codes included sentences or sentence fragments that represented data and concepts (Miles, 
Huberman, and Saldana, 2020). The coding process began by coding broad themes then coding with details. For example, if an interviewee described a lack of awareness of ADRD research opportunities, it was coded as "barrier, ability, system, awareness." Conversely, if an interviewee described a policy that led to funding partnerships, it was coded as "facilitator, ability, policy, funding, partnership." Most of the analysis was deductive, meaning the codes were analyzed with the notion that they may align with orienting concepts derived from the literature and theories (Miles et al., 2020). An inductive approach was also used to code and analyze themes that did not correspond with existing literature and theories to identify themes that emerged from the data organically and mitigate the possibility of coding those themes based on current literature rather than the interviews (Lapadat, 2010). The investigator was the single coder and the CAB interpreted findings by identifying what was most important, surprising, or missing in the interviews.

\section{Results}

Ten clinicians, researchers, and advocates were interviewed, with one interview removed from the analysis due to the interviewee discussing their experiences in a location outside of the study's geographic area. Nine interviews were ultimately included in the final analysis. The interviewees were employed by (8) or volunteered with (1), several health, research, or advocacy organizations of varying sizes and structures. Table 4.2 provides the characteristics of interviewees. 
Table 4.2: Interviewee Characteristics

\begin{tabular}{|c|}
\hline Investigator at large research organization \\
\hline Research associate at large research organization \\
\hline Research associate at small research organization \\
\hline Neurologist/investigator at large research organization \\
\hline Advocate at ADRD advocacy organization \\
\hline Support group facilitator and advocate \\
\hline Geriatrician at mid-sized health care organization \\
\hline Geriatrician at large health care organization \\
\hline Geriatrician at large health care organization \\
\hline
\end{tabular}

Table 4.3 summarizes the key findings, organized as thirteen subthemes across

five main themes: engaging with people with ADRD; policies and funding mechanisms; partnerships and collaborations; clinical research as an organizational value; and perceptions of ADRD clinical research participation. Subthemes are discussed in detail below.

\section{Table 4.3: Themes and Subthemes from Interviews}

\begin{tabular}{|c|c|}
\hline Themes & Subthemes \\
\hline $\begin{array}{l}\text { Engaging with people } \\
\text { with ADRD }\end{array}$ & $\begin{array}{l}\text { - Organizational roles and processes } \\
\text { - Alzheimer's Association as a tool for engagement }\end{array}$ \\
\hline $\begin{array}{l}\text { Policies and funding } \\
\text { mechanisms }\end{array}$ & $\begin{array}{l}\text { - Industry funding } \\
\text { - Policies affecting engagement with people with } \\
\text { ADRD }\end{array}$ \\
\hline $\begin{array}{l}\text { Partnerships and } \\
\text { collaborations }\end{array}$ & $\begin{array}{l}\text { - Community collaboration } \\
\text { - Multi-site and cross-sector collaboration and } \\
\text { partnerships } \\
\text { - Advisory boards }\end{array}$ \\
\hline $\begin{array}{l}\text { Clinical research as an } \\
\text { organizational value }\end{array}$ & $\begin{array}{l}\text { - Research as a priority } \\
\text { - Communication of research opportunities }\end{array}$ \\
\hline $\begin{array}{l}\text { Perceptions of ADRD } \\
\text { clinical research } \\
\text { participation }\end{array}$ & $\begin{array}{ll}\text { - } & \text { Fear } \\
\text { - } & \text { Shift in mindsets } \\
\text { - } & \text { Caregivers as gatekeepers } \\
\text { - } & \text { Motivation discrepancies } \\
\end{array}$ \\
\hline
\end{tabular}




\section{Theme 1: Engaging with people with ADRD}

The two subthemes related to Theme 1 are organizational roles and processes and the Alzheimer's Association. A summary of how they affect ADRD clinical research participation is provided at the end of this section.

Organizational roles and processes: Two of the three local ADRD research organizations were included in this study (the third did not respond to requests to be interviewed). Both research organizations additionally serve as cognitive health clinics, but there was a substantial difference in the size of the two organizations. The small research organization opened a few years ago, had a very small number of employees and was not actively recruiting for studies at the time of the interview. The large organization has over 100 employees, has been in operation for 30 years, and was recruiting for many studies. Both organizations recruit participants within and outside of their regular patient population, as described below:

Researcher: "Our primary way of informing them about our studies and enrolling them is word of mouth through our clinic. The doctors who see our patients often have research study discussions and they're not allowed to say, 'Hey, do you want to do this study?' But they can say, 'Are you interested in research?' ... Then we have certain approved flyers that we put up around. We also go and give community lectures [and] slide in some information about our studies."

Researcher: "We've done talks, we've had our physician do talks in the community before. We have done plenty of advertising, we've gone through the [newspaper], we have done radio advertising, but that did not work at all. The [newspaper] is actually where we've had the best turnout. We've done fliers and canvassing; it's kind of rare as to where you can find a place where you can canvass nowadays. A lot of people don't want you hanging up your posters and things like that, so kind of rare in where we can go with that, but I don't think we've had anybody coming in saying that they saw it from a library poster or something like that." 
The large research organization held weekly clinic meetings where clinicians discuss their patients, and a coordinator is present to advise if the person with ADRD or their caregiver might qualify for a study. Study coordinators are involved in, or oversee, all aspects of their specific studies, but generally are not familiar with other studies beyond those to which they are assigned. However, the organization added the role of a recruitment coordinator who is familiar with all of the organization's studies. This coordinator fields phone calls from interested community members or patients, lets them know which studies they may qualify for, and connects them with the appropriate study coordinators. Researchers regarded this recently-added position as beneficial to improving recruitment.

All of the healthcare providers interviewed served as the directors of their respective departments or divisions. Many of these interviewees noted that primary care providers often refer patients to geriatricians or neurologists in order to obtain an ADRD diagnosis, evaluation and treatment, and resources for both patient and caregiver:

Healthcare provider: "There are several of the general internists who have learned the basics of addressing cognitive impairment. They often still want us to see the patient to provide more education and support. But, some of the primary care providers say, "Oh, you have a memory problem? Okay, we need to refer you." So, it's variable as to people's ability or willingness to manage patients on their own."

One provider noted that with the increase of at-home DNA testing kits, they receive referrals from individuals who discovered they were at higher risk for Alzheimer's disease and want to be vigilant about reducing their risk or preparing for a diagnosis. However, the provider's patient panel ranged from those with no cognitive impairment to those who were no longer able to communicate. Conversely, another provider noted that 
they only work with frail older adults, over $60 \%$ of whom had a confirmed ADRD diagnosis.

Two of the nine interviewees were advocates for people with ADRD, one of whom held a professional role with the local chapter of the Alzheimer's Association. The mission of the Alzheimer's Association focuses on providing support groups, education, and resources for people with ADRD and caregivers, as well as advocacy and research. The second advocate was a former spousal caregiver who volunteered as a facilitator for ADRD support groups and held positions on advisory boards for caregiver and ADRD support policies in the local area.

Alzheimer's Association: Nearly every interviewee mentioned the Alzheimer's Association as a primary way they either recruit people with ADRD and caregivers or provide education, support, and resources for them. In addition to working directly with people with ADRD and their caregivers, the Alzheimer's Association also supports local community organizations. Their involvement was recognized as significant for one researcher whose work focuses on brain health and the local African American community:

Researcher: "[The Alzheimer's Association] has tables at [our] events, and the tables really feel like it's for the community, whereas sometimes you might have a table where researchers are just trying to get people ... the Alzheimer's Association, they're not doing research studies ... they have these materials about you know, what are 10 signs of Alzheimer's disease? What are some free online workshops that you can attend? Where are there caregiving classes or even just bringing the person you're caring for even to the Alzheimer's Association to the Portland branch and they can do art activities while you discuss with other caregivers? So all those types of things really feel like it's for the community instead of another ask from the community." 
However, one researcher noted that they no longer collaborate with the Alzheimer's

Association because the study staff did not reach their target populations:

Researcher: "[Investigator] used to be involved with the Alzheimer's Association and setting up booths after runs and things like that, and [investigator] just found that it really didn't benefit the people that needed it. So the people who were actually at the runs and things like that would be the younger cousin who's representing somebody who could benefit from the services, but they're not there, and then the information wasn't really being [transferred] to the people who really needed to hear it. So [investigator] didn't really find any benefit there for us or for any patients in that aspect."

Summary: Organizational processes and resources contributed to an organization's strategies for engaging with people with ADRD. Research organizations used clinic records and had different ways of finding non-clinic individuals who might be interested in research. Having a recruitment coordinator who can refer interested individuals to the appropriate study has been beneficial to study recruitment. Health organization

interviewees spoke mainly about their referral system for treating people with ADRD and caregivers. Some organizations had formal processes for referrals; others had more informal, flexible processes. The Alzheimer's Association provided options for many of the organizations, who in turn were able to refer individuals to the Alzheimer's Association's resources and support groups and reach people with ADRD. However, not every organization had the same level of success.

\section{Theme 2: Policies and funding mechanisms}

The two subthemes discussed for Theme 2 are industry funding and processes and policies for research recruitment. A summary of how they affect ADRD clinical research participation is provided at the end of this section. 
Industry funding: Researchers receive funding from a variety of sources, including nonprofits (e.g. Alzheimer's Association), government (e.g. National Institutes of Health) and internal funding, but industry sponsors (e.g. pharmaceutical companies) provided substantially more money. One researcher specifically stated that applying for nonindustry grants was not worth their time since they already receive a lot of money from industry sponsors. However, researchers also are required to adhere to their sponsor's specific standards. All industry-funded research activities and strategies, such as recruitment materials, compensation for study participation, or the use of a specific MRI machine, are dictated by the industry sponsor and any modifications require their approval:

Researcher: "Occasionally, with industry-funded trials, they're a little bit pickier about the kinds of advertising that we do. So any sort of patient-facing advertisement like a brochure or a flyer or even like a phone call, we need to have all of that approved by the sponsor as well as the IRB ... If we don't like it, we're like, "Oh. Well. I don't think the patient's really going to want to be in this trial if it says that." But if that's what the sponsor requires then we're kind of stuck [with] that. It might limit our engagement with people. We have had that happen before."

Researcher: "So the sponsor sends somebody to do [quality assurance testing] on one specific machine, so we can't even just send them to one facility, it has to literally be one specific machine that was [tested] by them, so that they're scanned under the same protocol each time on the same machine, really just anything to reduce variability. That would help, if we could send people to other places."

Researcher: "Usually, the sponsor has relationships with multiple vendors that we have to work with directly but we don't have contracts with directly. When I first started, that was nine years ago, we didn't have hardly any vendors. Maybe one for the MRI review or something. But, now, our latest trial has 10 vendors. All these different labs and PET scan review, PET scan dose ordering and MRI. There's a vendor for paying the subjects and there's a vendor for looking at photographs of subjects skin changes that are a risk factor in the trial. All kinds of different things. That makes it a lot more difficult." 
Policies for research recruitment: There are new federal policies that focus on diversity in research, such as the NIH's Inclusion Across the Lifespan policy (NIH, 2018), which went into effect in 2019:

Researcher: "With the new NIH policy... you have to now say why you're ending it at a certain age, your eligibility, like if you're saying age range is between 5090, why are you ending at 90? ... It just seems silly that so many studies end at 90 when that's precisely the group that's more likely to be affected by ADRD."

However, many interviewees were unable to identify specific policies that improve ADRD research participation. Other interviewees discussed policies that have been enacted but not utilized in the field. As previously mentioned, there was a recent NIH policy change to allow a single IRB for multi-site studies (NIH, 2016), but this policy change has not been utilized by the researchers interviewed for this study:

Researcher: "I think institutions tend to stay within their bounds of what they're used to doing. One area this has clearly shown its face is in the area of trying to enact a uniform, a single consent process, so I won't have 20 different IRBs reviewing the same consent but coming up with 20 different versions. The time and delay that just generates practically, versus having a common agreement that we'll defer to one central organization. It's been aspirational. It's still not happening. I still see just in recent experience with multi-center projects that we're supposed to use a central IRB or a single IRB. In practice, it still hasn't happened ... I think most of it is fear, it's liability."

Conversely, some healthcare providers described billing and Medicare policies that made it easier for them to see patients with ADRD:

Healthcare provider: "There has been a change in the last five years, since we took on this consult model, where we realized that we couldn't be like a primary care clinic where a provider typically sees twenty, twenty five patients a day. That doesn't work at geriatrics just because they have special needs, they need more time. We in our consult clinic have a ninety minute visits for the initial consult with the provider .... Because we have these longer appointments with our coding has changed in that we code prolonged services, so we code by time which is different from what a typical primary care clinic codes for. This allows us enough time to do evaluations." 
Summary: Policies and funding mechanisms were described as playing a large role in affecting ADRD research participation. Policies were enacted to streamline the study start-up process or incentivize inclusive research, but some policies are deemed ineffective in practice. Industry funding offered a strong balance between having additional funds for research study activities, but came at a cost of additional barriers, potentially affecting recruitment or ability for individuals with ADRD to participate.

\section{Theme 3: Partnerships and collaborations}

Three subthemes are discussed in this section: collaborating with the community, collaborating with non-community organizations, and advisory boards. A summary of how these subthemes affect ADRD clinical research participation is provided at the end of this section.

Collaborating with the community: Some interviewees spoke about sharing information with community groups through presentations or flyers, whereas others described collaborations for referrals or research recruitment. One researcher worked with an African American community organization that serves as an advisory board for some of the research organization's studies and also has a designated workflow to determine with which investigators to work and share resources with. Some interviewees articulated that any work with community groups or members was volunteer time away from their job; one noted that she was required to make up the time:

Healthcare provider: "I honestly devoted hours and hours and hours to trying to develop processes and education. But, over time, it's been hard to remain enthusiastic because any time I spend working on things like that, comes out of my own personal time. And people don't seem to understand that I'm not funded to attend two to three hour long meetings. And if I spend time in a meeting, then I have to make up for that time at the end of the day later." 
Researcher: "It is a challenge, particularly for minority research, where the key element to successful research in minority communities is establishing a long standing relationship. In order to do that, it takes resources. You can't really go out and fund a grant to create those relationships. It's basically volunteer time in a way ... develop those relationships. Then hopefully they just get established, and then you open the door to a more formal project or study."

Collaborating with non-community organizations: All interviewees spoke of partnering with other healthcare or government agencies to provide resources for people with ADRD or caregivers. Additionally, researchers spoke of collaborations with other researchers or professional research organizations to work on projects together. As previously mentioned, one researcher noted that despite attempts to have one uniform process for these collaborations, each organization ends up following their own rules out of fear of liability, often resulting in delays in study start-up and recruitment.

Additionally, collaboration with vendors led to partnerships to streamline study start-ups:

Researcher: "There's also a new partnership that we have called the Alzheimer's Clinical Trial Consortium. ACTC. They are focused on helping clinical trials get started up a little bit faster so they're an umbrella consortium ... [to] help with all of the startup. Because start up for a study takes so long with the negotiations and contracts ... I would say our longest trial that went from very beginning to completely ready to go was one year. I would say six months at a minimum. Very rarely do we get a trial up and running in less than six months. There are just a lot of pieces and negotiations and that takes a while."

Advisory boards: Respondents who spoke of advisory boards had mixed feelings. Some felt that they provided opportunities for community empowerment and an opportunity to learn from the experts. Others felt they did not truly capture community needs:

Researcher: "If we have a meeting, I make sure that we don't have 7 [researchers] and 3 community members on the other side of the table ... we are conscious about power imbalance. And then we always approach them as 'you're the experts. 'So we always want to approach it as 'we want to learn from you' because people will always think 'oh I need to give them the answer they're 
looking for. What if I don't know about what they want?" ... It's them giving to us, when really...we want to say, 'look, you're the experts, help us learn from you.' And so it's putting them in a position of knowledge and, you can even say, empowerment. But whatever they have to tell us is what we want to hear."

Researcher: "Those are challenging, because I think they sound good, but if you think about it, how can one person from a community represent the community? I mean, you take it as input, but I think that it's hard to get the voice of a community from a single community representative. We've had this experience where there's a person from the community who is quite vocal and has a view that we're pretty sure is not necessarily the majority view, but they're vocal and adamant... you take it as input, but I think that it's hard to get the voice of a community from a single community representative."

Some respondents questioned the membership of people with ADRD on advisory boards

given the nature of their disease:

Healthcare provider: "Some of the people who go to the participant advisory council meetings, I'm sure have dementia. The biggest challenge inherent to dementia ... is apathy ... it just looks like they don't do anything. And they don't care about anything. And they don't say anything. Which is all true...So, I think having dementia itself is going to stand in your way. I mean, there are really exceptions. There's a person here or there who's, for whatever reason, the one out of four who doesn't have that syndrome predominantly."

Healthcare provider: "For the things I've done, we haven't included individuals with dementia ... with dementia, people often lack insight, they often have enough memory impairment that they won't remember a previous conversation. So, you can't build on what you've done previously and it's just a much more limited interaction."

Only one interviewee, an advocate, described actively recruiting people with ADRD for their advisory boards and advocacy work:

Advocate: "We have our own board. We have people living with cognitive impairment and change ... They are there because they're an expert. They know what it means to live with this disease every single day. They know what it means to care for someone's disease or to watch their husband change before their eyes. They know what it means. They're an expert and that's who policymakers want to hear from." 
Summary: Collaborations and partnerships affected relationships between organizations and people with ADRD, as well as partnerships between organizations. Some organizations valued the role that community members have as experts, others did not believe that community members are experts, or had negative thoughts. This is additionally true for people with ADRD -- while some thought their expertise was integral to the research process and decision-making, others believed that having people with ADRD involved was not good practice due to the nature of the disease.

\section{Theme 4: Clinical research as a value}

The two subthemes discussed for Theme 4 are research as a priority and conversations about research opportunities. A summary of how they affect ADRD clinical research participation is provided at the end of this section.

Research as a priority: For the researchers and some advocates interviewed, research was viewed as a high priority and incorporated in all aspects of their work:

Researcher: "I think doing dementia clinical work day-in-day-out will burn you out. It certainly would burn me out immediately. Being able to do research ... helps me feel like, well at least I'm trying to move the needle ... It's not going to get better unless we understand how to fix it. That's why I think it's so important, and that's why I do it."

For the healthcare providers and other advocates interviewed, research was not a high priority because their organizations focused on providing healthcare, education, or other support resources. For example, one provider spoke of a research project that they planned to collaborate on with a research organization, but had to end their participation:

Healthcare provider: "It's become an entirely [research organization]-based project because of lack of support [at healthcare organization] for the research components ... Originally we designed it as a project that would be based in both institutions and we've now shifted it to be entirely [research organization]-based. 
It's not to say that our patients ... couldn't participate but we can't recruit and enroll them here."

Conversations about research opportunities: Two of the three ADRD healthcare providers who do not conduct ADRD clinical research reported on organizational policies that banned or discouraged active sharing of information about research opportunities:

Healthcare provider: "Because of the population that we treat, [the Centers for Medicare and Medicaid Services] has it in our regulations that we are not allowed to enroll our participants in research ... And it's really because we're a capitated plan ... we control nearly everything about our participants. So, if we don't approve it, and we don't transport them to it, and we don't provide it, they don't get it." 2

Healthcare provider: "I suppose we could say, 'There are some research projects over at [research organization]. If you're interested you can contact this person or this phone number.' But that's on the edge of what we're supposed to do."

Conversely, many respondents from research organizations reported that providers

typically discussed research opportunities with patients as part of their care plans:

Researcher: At the end [of the first visit], you say, "Well, you could take this drug. You could do this. You really need to have this test though." Then of course we want to bring up, "This is a research center. If you would be interested, we would love to have you consider being involved in a research study." Then we would say, we usually have in mind what that might be based on what we've heard. It might be a caregiver study, it might be a drug study, it might be enrolling in the [organization] as a cohort person."

Additionally, some responses indicated that there was a large segment of community members who were not aware of opportunities or responsive to researchers' recruitment efforts:

Advocate: "We need to hear about it more. I mean, if I don't know about it ... If I had some pamphlets or if [researchers] like you want to come and I introduce you

\footnotetext{
${ }^{2}$ Interviewee sees patients through the Program for All-Inclusive Care of the Elderly (PACE), a capitated comprehensive health and long-term service program for dual-eligible, frail, community-dwelling, older adults. (Centers for Medicare and Medicaid Services, 2011)
} 
and say this is what you're doing, and if anybody wants to talk to you, meet you after ... I think it needs to be more of a priority. But like I said, I've never had any information on it."

Researcher: "It's so easy to get the easy people, the people in our networks, and if our network isn't very diverse ... and the people who have been in other studies keep coming back, and then it's easy to say 'oh, well it's too hard to get that population.' I think it just means we're not planning ahead enough, we're not working hard enough, we're not using our networking skills and community building to reach those people, or maybe the way we're approaching those people is not the right way culturally to approach those people."

Summary: An organization's perceived value of clinical research affected the likelihood of a conversation about clinical research opportunities and ultimately affected awareness and the ability to participate in research. Some organizations perceived clinical research to be of such low value that they have policies in place to avoid conversations, whereas others may consider research to be as valuable as other courses of action in a care plan. Interviewees also noted that there were missed conversations with many community members, suggesting that research organizations may need to rethink recruitment strategies.

\section{Theme 5: Perceptions of clinical research}

Four subthemes are discussed in this section: fear, shift in mindset, caregivers as gatekeepers, and motivation discrepancies. A summary of how these subthemes affect ADRD clinical research participation is provided at the end of this section.

Fear: Interviewees discussed knowledge of some providers appearing to be afraid to give an ADRD diagnosis, which could affect the ability to participate in studies that require an ADRD diagnosis. Others suggested that people with ADRD may be afraid to participate 
in research because it could lead to being identified as someone with ADRD, or may be afraid that research participation will disrupt their routines:

Healthcare provider: "Dementia research is in the same place cancer research was in the 1950s. When cancer was a terminal illness, there were no treatments, there were no disease modifying effects, and we didn't tell people they had cancer. And that's exactly how it is for dementia. We don't tell people they have dementia."

Healthcare provider: "There is a fear factor ... fear of the unknown as to what's going to happen to them [in the study] but also having other people know about their cognitive issues. We see that a lot of people who have been really high functioning they mask cognitive issues. There's that really, you know just coming out and sharing that with other people. There's a concern there."

Shift in mindset: Many interviewees advocated for reframing ADRD in order to remove the stigma around it. One suggestion included viewing ADRD and brain health as a public health issue rather than an individual one. Another suggested re-marketing ADRD similarly to what has been done in describing cancer, such as using the terms "fighters" and "champions" to describe people with ADRD. Yet another suggestion was to recognize the resilience that older adults have in the face of many medical changes, and challenge the notion that nothing can be done to help the individual once an ADRD diagnosis has been made:

Advocate: "One of [the organization's policy goals] is increasing public awareness and early detection and diagnosis around Alzheimer's disease ... really taking a look at Alzheimer's disease that leans towards public health, that this is a public health crisis. It's not just a disease affecting people that are aging, but everybody is affected by it."

Healthcare provider: "I think on the provider's side ... it's just uncertainty and feeling like there's nothing for them to do. So why make the diagnosis? Because they could be wrong. When in fact, there are things to do. Get your affairs in order, advanced directives, you know? There are lots of things to do. Do a bucket list..." 
Caregivers as gatekeepers: Most interviewees described the important role that

caregivers play as a major factor affecting research participation, given the requirement

that study partners also participate in ADRD research:

Researcher: "Sometimes the participant is really gung-ho and ready and wants to be involved and the caregiver just doesn't care, so that's really unfortunate and we see that often enough, where it's the caregiver dropping the ball, just not following through, not getting back to us for scheduled appointments, and just lost through follow up, basically, which is really unfortunate."

When asked to provide recommendations to improve ADRD research participation, many focused on improving the caregiver's ability to participate in research via flexible scheduling and locations, or providing education or support for the caregiver:

Healthcare provider: "If caregivers could get support ... sometimes maybe there's a small stipend or agreement that people get from research participation. But sometimes it could be in terms of supporting caregivers. They might see this as a plus instead of just a monetary part of it."

Motivation discrepancies: Research coordinators and advocates found it easy to

describe the high motivation for people to participate in research:

Researcher: "For Alzheimer's disease studies, folks are really motivated to participate. They will go to great lengths to participate in our trials and, sometimes, we're even surprised. We're like, "Wait a second. You live two hours away and you're willing to come in every two weeks for six months? Wow." That's really surprising. But people are just so motivated to find a cure for Alzheimer's disease that they often say, "I don't want my children to go through this or my grandchildren." So they're really motivated to help us."

Researcher: "This is a demographic that is really eager to participate because they're worried ... they're not as good as they once were before ... so I think we do get a lot of people who are willing to be applicable for the study but I don't think they're impaired like they think they are."

Conversely, healthcare providers found it easier to identify specific reasons why people with ADRD would be not be motivated to join research studies: 
Healthcare provider: "And the reality is, yes, if you are participating in a study, you are not really going to benefit yourself. It's about benefiting others."

Healthcare provider: "I do think that for a lot of research you can appeal to people's altruism. And the idea of helping other people like you. But, I'm not sure that that's a very significant motivator with people who have dementia."

One researcher noted that there are more concerns about participating in research due to the negative historical relationship between researchers and specific communities, so participants want to know that the research is giving back to their community:

Researcher: "I send out updates all the time ... where we're saying 'hey we got our abstract accepted; we're presenting on a national scale, people in Washington DC are caring about the research you participated in' and we let people know that that research assistants that were helping us out, 'because of you participating in our study and their experience working with you in research, they've been accepted to these grad schools' and so we just keep connected that way, and so that's like 'oh hey, you know, we have a conversation going on about research instead of a constant ask [of participants to do something for us]."

Summary: These interviews highlight that perceptions of clinical research vary from person to person, as do perceptions of perceptions. Fear, personal and public perceptions of ADRD affect motivation. Organizations also affect motivation and ability to tell patients about ADRD research opportunities. All of these factors affect the ability of a person with ADRD to participate in research.

\section{Discussion}

These five themes and thirteen subthemes illustrate organizational, system, and policy level factors affecting ADRD research participation. The thematic findings are discussed below in relation to the three levels of factors, i.e, organization level, system level, and policy level, which were described in the literature review. 


\section{Organizational factors}

Interviewees described several organizational factors affecting ADRD clinical research participation, as presented in Table 4.4. These factors can be further evaluated using organizational theories, such as contingency theory.

Table 4.4: Themes in Relation to Organizational Factors

\begin{tabular}{|c|c|}
\hline Organizational Factors & Themes \\
\hline Data and compliance oversight & $\begin{array}{l}\text { - } \quad \text { Engaging with people with ADRD } \\
\text { - } \quad \text { Policies and funding mechanisms } \\
\text { - } \quad \text { Partnerships and collaborations } \\
\end{array}$ \\
\hline Eligibility criteria & $\begin{array}{ll}\text { - } & \text { Engaging with people with ADRD } \\
\text { - } & \text { Policies and funding mechanisms } \\
\text { - } & \text { Perceptions of ADRD clinical research } \\
& \text { participation } \\
\end{array}$ \\
\hline Recruitment strategies & $\begin{array}{ll}\text { - } & \text { Engaging with people with ADRD } \\
\text { - } & \text { Policies and funding mechanisms } \\
\text { - } & \text { Partnerships and collaborations } \\
\text { - } & \text { Clinical research as an organizational } \\
& \text { value }\end{array}$ \\
\hline $\begin{array}{l}\text { Relationships with people with ADRD/ } \\
\text { caregivers }\end{array}$ & $\begin{array}{l}\text { - Engaging with people with ADRD } \\
\text { - Partnerships and collaborations, } \\
\text { - } \text { Clinical research as an organizational } \\
\text { value } \\
\text { - Perceptions of ADRD clinical research } \\
\text { participation }\end{array}$ \\
\hline IRB/Security protocols & - $\quad$ Policies and funding mechanisms \\
\hline
\end{tabular}

Contingency theory posits that organizations can modify some of their rules and processes to best address environmental needs (Lawrence \& Lorsch, 1967; Galbraith, 1973). Organizational roles and processes play an integral role in initial and continued engagement with people with ADRD. At the larger research organization, study coordinators generally stick to their own studies, but there is a central staff person who can match the abilities and interests of patients and community members with study 
opportunities for which they may be eligible. In the smaller research organization, all staff worked on every study. In healthcare organizations, clinician referrals play a large role in diagnosis, treatment, and provision of resources to people with ADRD and their caregivers. Notably, there is a wide variability of primary care providers' willingness and ability to discuss a possible ADRD diagnosis with their patients. Some organizations automatically refer a patient with cognitive impairment to another specialist, in other cases, primary care providers can use their own discretion.

Both research organizations used similar research strategies for recruitment through the clinic, community talks, media advertisements, and flyers, with differing levels of success. However, the same group is often people participating in multiple studies within the organization over a variety of years because they are more inclined to participate as compared to someone who has never participated before. The interviews highlight that the methods used to recruit in the Portland area need to be revisited to avoid re-enrolling the same network of people. Recommendations derived from the interviews include presentations at ADRD and caregiver support groups, making materials reflective and culturally responsive to the community that researchers wish to work with and providing additional incentives for caregivers. These recommendations are similar to what has been described in the literature (King et al., 2007; Uybico, Pavel, \& Gross, 2007; Huang \& Coker, 2010; Monson et al., 2012; Newington and Metcaffe, 2014; Black et al., 2017).

The finding that clinical research ranks as a higher priority to research and advocacy organizations as compared to healthcare organizations was unsurprising, but it 
was unexpected to learn that some healthcare organizations had policies in place to restrict conversations and discussions about clinical research. Two of the interviewees worked at organizations that are completely or primarily funded by federal dollars, which means they are more restricted in the modifications they can make to adapt to environmental needs. As previously mentioned, most people believe they will hear about research opportunities from their providers (Anderson et al., 2018), so this substantially influences the ability of people with ADRD to learn about research opportunities.

In addition to roles and processes that can be adjusted based on environmental needs, contingency theory is useful for articulating what cannot be changed. For example, researchers must adhere to IRB, security, and data compliance protocols that are determined by their larger organization, funder, or governing body's policies. While this takes time and funding resources away from other research activities, including recruitment, these are processes that cannot be adjusted at the researcher or departmental level. Similarly, certain eligibility criteria, such as excluding those without study partners, those who do not have the required memory scores, or those who take contraindicated medications, are non-negotiable due to safety regulations. However, some criteria could be modified, such as age, distance from the study site, and in some cases, willingness to travel to the clinic, in order to address community member needs.

\section{System level factors}

The schools of organizational theory of open rational systems (Scott, 1987) and contingency theory (Lawrence \& Lorsch, 1957; Mintzberg, 1983) can also be used to describe system level factors. These factors are presented in Table 4.5. 
Table 4.5: Themes in Relation to System Level Factors

\begin{tabular}{|c|c|}
\hline System level factors & Themes \\
\hline $\begin{array}{l}\text { Communication about research } \\
\text { opportunities }\end{array}$ & $\begin{array}{l}\text { - } \\
\text { - } \text { Partnaging with people with ADRD } \\
\text { - } \text { ADRD clinical research as an organizational } \\
\text { value } \\
\text { - Perceptions of ADRD clinical research } \\
\quad \text { participation }\end{array}$ \\
\hline Clinician training and interest & $\begin{array}{l}\text { - Engaging with people with ADRD } \\
\text { - } \quad \text { Clinical research as an organizational value }\end{array}$ \\
\hline Community engagement & $\begin{array}{ll}\text { - } & \text { Engaging with people with ADRD } \\
\text { - } & \text { Partnerships and collaborations } \\
\text { - } & \text { Clinical research as an organizational value }\end{array}$ \\
\hline Structural elements & $\begin{array}{l}\text { - Engaging with people with ADRD } \\
\text { - } \begin{array}{l}\text { Perceptions of ADRD clinical research } \\
\text { participation }\end{array}\end{array}$ \\
\hline
\end{tabular}

Non-community collaborations, such as those with other healthcare clinics, research organizations, or government and advocacy agencies were discussed more frequently than community collaborations. Some collaborations and partnerships aimed to enhance recruitment by streamlining the study start-up process, such as negotiating contracts between organizations and vendors in order to begin recruiting and enrolling faster. Others aim to educate the local community about clinical research, including ADRD studies. Interviewees from both research organizations noted that these partnerships had recently begun. These partnerships were developed based upon a need to ultimately facilitate research participation, and processes were developed to achieve this goal.

The Alzheimer's Association can be considered at the system level, as it plays a key role in facilitating engagement with people with ADRD and their caregivers across 
multiple organizations and community settings. Many providers and researchers work with the Alzheimer's Association to recruit, share information, and offer support, without the Association apparently asking for anything in return from community members. Experiences were reported as generally beneficial; however, it is important to note that one researcher had negative experiences tabling at the Alzheimer's Association's community events as study staff were not able to reach the study's target population. However, it is also possible that the study staff needed to use different methods to reach these populations through the Alzheimer's Association, such as presenting study opportunities at support groups (at the discretion of the group facilitator) or posting recruitment announcements to the support group's mailing list.

Transaction cost theory can also be used to articulate system level factors. One example is the use of advisory boards that include community members. Many interviewees seemed dismissive of community representation, especially when it was inclusive of people with ADRD. Numerous negative symptoms of ADRD were discussed, negating the fact that ADRD is a progressive disease, and many people can participate in advisory boards at various levels, with and without accommodations, until they are no longer interested in doing so. This is explicitly shown through the advisory boards led by the Alzheimer's Association and DeNDRoN, where the expertise of people with ADRD is recognized and brought in for decision-making processes (Iliffe, McGrath, \& Mitchell, 2013; Plunkett, 2017).

Another example of how transaction cost affects research recruitment at the system level is the concept of using a central IRB for multi-site studies. The relatively 
new NIH policy was intended to reduce time-consuming bureaucratic practices and streamline some study processes, yet organizations continue to use their own IRBs because the cost of fear and liability outweigh the possible benefits of timeliness and centralization. The transaction cost of using a central IRB is higher than the cost of continuing to use single site IRBs, so organizations make the decision to continue to use single site IRBs. This leads to a longer delay before recruitment, scheduling visits, and participation can begin.

A third example is the perceived motivation and ability for people to join studies. Researchers found that many people with ADRD and caregivers were motivated to join studies from an altruistic perspective of benefitting society as a whole; the benefit of hope and helping others is worth the cost of frequently traveling long distances or giving a lot of time to be part of a study. However, healthcare providers seemed to have trouble identifying motivations for participating in ADRD research, instead of focusing on the high costs of research participation. The researchers and healthcare providers perceived these transaction costs differently -- researchers believe the transaction cost of participating in research is worth the benefit, whereas healthcare providers felt the transaction cost was too high.

\section{Policy level factors}

Social construction and PFT can help describe the policy level factors affecting ADRD clinical research participation that are presented in Table 4.6. 
Table 4.6: Themes in Relation to Policy Level Factors

\begin{tabular}{|c|c|}
\hline Policy level Factors & Themes \\
\hline Data and compliance oversight & $\begin{array}{l}\text { - } \text { Engaging with people with ADRD } \\
\text { - } \quad \text { Policies and funding mechanisms } \\
\text { - } \quad \text { Partnerships and collaborations }\end{array}$ \\
\hline IRB/Security protocols & - $\quad$ Policies and funding mechanisms \\
\hline Recruitment and diversity policies & $\begin{array}{ll}\text { - } & \text { Engaging with people with ADRD } \\
\text { - } & \text { Policies and funding mechanisms } \\
\text { - } & \text { Clinical research as an organizational value }\end{array}$ \\
\hline Dissemination policies & $\begin{array}{l}\text { Perceptions of ADRD clinical research } \\
\text { participation }\end{array}$ \\
\hline Funding opportunities & $\begin{array}{l}\text { Policies and funding opportunities, } \\
\text { Partnerships and collaborations, } \\
\text { - Clinical research as an organizational value }\end{array}$ \\
\hline
\end{tabular}

Social construction explains the prominent role that reluctance and fear have in ADRD research participation. Providers are reluctant to diagnose a person with a progressive disease with no known prevention, treatment, or cure, and individuals may fear being identified as a person with ADRD due to socially constructed perceptions of people with ADRD.

Schneider et al. (2014) places socially constructed groups into four categories. People with ADRD would fall into the "dependent" category -- those who are positively viewed by society but would be considered politically powerless. Policy designs and public perception have deemed them worthy of benefits, but they are still viewed as powerless and unable to contribute to decision-making. For example, many researchers and healthcare providers seemed dismissive of community representation in research, especially when it was inclusive of people with ADRD. Numerous negative symptoms of ADRD were discussed, negating the fact that ADRD is a progressive disease, and many 
people could join advisory boards with varying levels of participation, with and without accommodations, until they are no longer interested in doing so. However, other researchers and advocates who viewed people with ADRD as "experts" led to more decision-making opportunities, highlighting the capabilities that people with ADRD do have. Additionally, while some researchers may view caregivers as "contenders" who have decision-making capabilities but are negatively perceived, the majority of interviewees viewed them as "advantaged", having positive views and recognizing their decision-making capabilities, which may explain why many suggested additional resources or incentives for caregivers to act as study partners for ADRD research.

Social construction may also describe the perceptions and values that are placed on ADRD research participation itself. Some organizations, particularly those primarily funded through the federal government, had policies in place to not discuss ADRD research opportunities or did not provide funding for research projects. They may perceive ADRD research as Contenders. However, advocacy groups and research organizations heavily promoted research opportunities, particularly the Alzheimer's Association, which is the third-largest funder in the world (Alzheimer's Association, 2019a). This is also evident through Congress's bipartisan decision to earmark over two billion dollars for ADRD research, and the NIA's strategy for improving ADRD research participation (Hodes, 2018; NIA, 2018).

For example, Congress's decision to provide $\$ 2.3$ billion dollars for ADRD clinical research increases the number of grants that a research organization can potentially be awarded, increasing the number of studies an organization can conduct, the 
length of time they have to conduct the research, the number of research staff they can hire, and the compensation they can offer a research participant. Similarly, the NIH's Inclusion Across the Lifespan policy removes exclusion criteria that often restricted adults over the age of 85 from joining ADRD research (NIH, 2017a). This new rule increases the ability to participate in research for a group of people who are substantially more likely to be diagnosed with ADRD. Conversely, recruitment procedures that do not use appropriate recruitment materials or networking opportunities can negatively affect the ability and motivation for people of color to participate in research. The NIA's new R24 grant, Examining Diversity, Recruitment and Retention in Aging Projects, provides funding opportunities for research organizations for relationship-building and community collaboration to develop recruitment strategies needed to increase the ability and motivation of diverse populations to participate in ADRD research (NIA, 2018).

PFT can also be applied to the balancing act of industry-sponsored studies. Organizations receive more money when funded by industry, but all activities, processes, and collaborations are dictated by these sponsors. This finding is important because it identified several barriers to recruitment. Though additional funding initially presents as a way to increase research participation through extra money for recruitment activities, several other procedures lead to a negative impact on an individual's ability and motivation to participate in research. These include time delays before the study can begin recruitment, the time needed to send participants or test results to a specific scan or lab vendor, the travel delays potential participants may experience traveling to a specific vendor, or specific recruitment materials that do not engage the intended communities. 
PFT also recognizes that policy feedback from past policies affects future policies (Skocpol, 1992), so it is possible that industry sponsors may modify their policies based on coordinator or community feedback. Policy feedback is evident in other examples of findings. For instance, the NIH's policy to streamline the multi-site study start-up through a central IRB was created based on the feedback that studies were being delayed.

However, with the realization that study sites are not being utilized as intended due to fear and liability, modifications will need to be made to ensure that fear and liability are combatted and the policy's outcomes are achieved as intended. Another example is the NIH's Alzheimer's Clinical Trial Consortium, which an interviewee noted was established due to the long delays organizations underwent in getting studies launched (Balintfy, 2017).

\section{Conclusion}

The purpose of this study was to identify organizational, system, and policy level factors affecting participation in ADRD clinical research in the Portland, OR metropolitan area. This research has several important findings. One notable finding is the discussion on enacted policies that have not made their way to practice. Despite attempts by the NIH and CMS to streamline research studies by allowing a single IRB for multi-site studies and provide care planning to people with ADRD and their caregivers, neither of these policies has resulted in the intended outcomes. Researchers noted that it is difficult to fully implement these policies due to organizational concerns, and advocates noted that research needs to be conducted to find out why healthcare providers have not taken full 
advantage of these policies. A second major finding is the lack of awareness that people with ADRD and caregivers have of research opportunities. All of the healthcare providers, with the exception of the dual healthcare provider/investigator, discussed the rarity of telling people with ADRD about research opportunities. In some cases, this was due to a lack of provider knowledge, but in others, it was due to organizational directives to not discuss or a provider's personal preference. Further, these opportunities are not being shared in places where people with ADRD and their caregivers are likely to congregate, such as support groups or social activities for people with ADRD, or may not be shared in a culturally appropriate way. A third major finding is that while some interviewees discussed providing educational presentations and seminars to teach community members, they were often hesitant to join community collaborations or formal partnerships, and many were wary of including people with ADRD in their advisory boards. This appears counterintuitive to a central theme in the NIA's strategy to increase ADRD research participation, that of local community collaboration (NIA, 2018).

There are several limitations to this study. First, the sample size was small $(n=9)$ and not fully representative of one metropolitan area. Though it was designed to be organizationally representative, all but one of the healthcare providers were geriatricians and there were no providers who specifically focused on primary care. This is because the investigator recruited interviewees based on referrals from key informants and community advisory boards, and nearly all provider referrals were for geriatricians. Further, one of the three local ADRD research organizations did not respond to requests 
for interviews. The study also did not include interviews with local county government employees despite the fact that county governments in the Portland metropolitan area provide services for people with ADRD and caregivers. The investigator was not referred to county employees. These county agencies were discussed by advocates, but not by providers and researchers, so it is possible that the services provided by the counties are unknown to local health and research organizations.

Second, these findings are specific to the Portland metropolitan area, and while they may be contextually relevant for similar communities, they are not necessarily generalizable. Lastly, there is always the risk of bias. Interviewees may have censored themselves due to the audio-recording of interviews and may have focused on what they perceived the interviewer would want to hear, resulting in response bias. The investigator mitigated the likelihood of confirmation bias by writing field notes immediately after each interview, a conscious effort to self-assess when analyzing codes and themes, and CAB member-checking of analyses. Furthermore, while there are no conscious biases due to the researcher's identity, it is important to acknowledge that subconscious biases are possible due to her identity as a female academic who is a proponent of ADRD research and community inclusion.

Several recommendations can be made based on the study's findings. First, research organizations' strategies may benefit from modifications to reflect community needs. Based on the interviews, this could include presentations at ADRD and caregiver support groups, making materials reflective and culturally responsive to the community that researchers wish to work with, and providing additional incentives for caregivers. 
This also applies to industry sponsors who may be receiving suggestions from coordinators or community members about best recruitment strategies but continue to adhere to their original processes.

Second, research could be enhanced by identifying why some health organizations have policies in place that discourage healthcare providers from speaking about ADRD research participation with their patients. It is important to note that this may be due to policies that are outside an organization's control, such as external funding mechanisms. In such cases, it would be beneficial to explore why the external funders or policymakers perceive ADRD research in a negative manner and to determine ways to assuage these perceptions.

Third, organizations may benefit from recognizing the expertise that people with ADRD can offer. Rather than excluding them from conversations, people with ADRD can be empowered through an organization's inclusive decision-making processes, such as including them on advisory boards. This inclusion may lead to a change in the social construction of people with ADRD.

Fourth, a shift in the social mindset needs to occur in order for people with ADRD to become empowered and reduce stigmatization of ADRD. This may be difficult to accomplish, but these interviews highlighted the numerous ways that the shift in mindset can be perceived. Social research could be conducted to identify what kind of shift would be most feasible and effective.

Fifth, funding opportunities for developing community collaborations should be utilized. Several interviewees described these collaborations as volunteerism on their 
part. The NIA offers funding opportunities for this purpose, and other opportunities may exist through nonprofits and philanthropies. However, only one interviewee noted that such a grant existed. Utilizing these opportunities can provide incentives for organizations to collaborate and may provide greater opportunity for people with ADRD and their caregivers to participate in decision-making processes.

Finally, caregivers should be given greater consideration in the design phase of ADRD research. As study partners, they are essential to research participation. Researchers should be willing to accommodate their schedules and needs, such as requiring fewer in-person visits, or provide resources or supports specific to the caregiver, and these efforts may help in increasing their ability and motivation to participate in ADRD research.

This research study identified organizational, system, and policy factors affecting ADRD research participation in the Portland metropolitan area. These were identified through interviews with local researchers, healthcare providers, and advocates, and the application of various organizational and policy frameworks to contextualize the themes into organizational, system, and policy level factors. Identifying how these factors affect the decision of a person with ADRD to participate in research is an important first step to increasing participation rates. Based on these factors, strategies can be developed and implemented to improve the ability and motivation of people with ADRD to join research studies in the Portland metropolitan area. This study should be repeated in a larger context or as pilots in other communities to determine contextual relevance and generalizability for other areas. Further, implemented strategies should be routinely 
evaluated to determine their feasibility and effectiveness. No matter the steps, researchers should consider the larger picture -- this is not just about finding a way to increase participation in ADRD clinical research studies, but more importantly about finding a prevention, treatment, or cure for ADRD. 


\section{CHAPTER 5: PERSPECTIVES OF PEOPLE WITH DEMENTIA AND CAREGIVERS ABOUT DEMENTIA CLINICAL RESEARCH}

\section{Introduction}

Nearly seven million people in the United States live with Alzheimer's disease and related dementias (ADRD), costing the nation over 236 billion dollars (US) per year in direct medical costs (NIA, 2018; Alzheimer's Association, 2019a). These numbers will increase as our population ages. Many healthcare professionals, policymakers, and those affected by these diseases agree that rapidly increasing ADRD prevalence rates (and subsequent rising costs) coupled with the lack of clinical solutions are creating a medical, economic and emotional crisis (Collins et al., 2018; Alzheimer's Association, 2019a; Gates, 2017). To combat this crisis, the United States Congress allocated 2.9 billion dollars for ADRD research in 2019 with the goal of finding solutions for prevention or treatment by 2025 (Alzheimer's Association, 2019b). Advancements in ADRD research are in part contingent on research participation by individuals with an ADRD diagnosis and their caregivers (Rollin-Sillaire et al., 2013; Law, Russ, \& Connolly, 2014; Grill \& Galvin, 2014; Black et al., 2016; Gates, 2019). To effectively utilize this historic funding, efforts to increase participation in ADRD clinical research are needed.

Individuals with ADRD face additional barriers to research participation as compared to research for other diseases. Only 10-27\% of people with ADRD are eligible to participate in research due to comorbidities, incompatible medications, low cognition scores, and frailty (Grill \& Galvin, 2014), so there are substantially fewer individuals 
with ADRD who would be eligible to become participants before even considering the person's interest in a study. Additionally, study partners are required to participate with the individual with ADRD to ensure protocol adherence and appointment attendance, further complicating the process (Karlawish et al., 2008; Watson et al., 2014; Grill \& Galvin, 2014; Cary et al. 2015; Black et al., 2017; NIA, 2018).

In this paper, three frameworks are considered to better understand the barriers and facilitators of engagement in clinical research by people with ADRD. Two frameworks, transaction cost theory and principles of civic engagement, can be used to conceptualize factors affecting ADRD clinical research participation. A third framework, contingency theory, can help to describe how organizations adjust their rules and processes to reduce the transaction costs for potential participants in ADRD research.

\section{Transaction Cost Theory}

The decision to participate in dementia clinical research can be partially explained by transaction cost theory; pioneered by Williamson (1975), this theory can be used to illustrate the financial and other costs of enrolling in a study. Transaction cost theory suggests that every transaction between two parties (both within and among organizations or groups) is subject to a cost. The decision to participate in an ADRD clinical research study is a transaction between a person with ADRD and a research organization, and the costs (both real and perceived) of the transaction affect the decision. The theory has four components that are based on market principles.

The first component, uncertainty, reflects changes beyond the control of the organization (Williamson, 1975). In the context of a research organization trying to 
engage and recruit people with ADRD and caregivers into studies, examples include symptom recognition and a timely ADRD diagnosis (or lack thereof) from a healthcare provider. In these cases, a person may not seek out an ADRD study if their cognitive impairment is misdiagnosed or misattributed, which may be the case for up to $60 \%$ of all people with dementia (Lang et al., 2017). Uncertainty may also refer to indirect knowledge transfer of study opportunities from healthcare providers and community organizations to people with ADRD and their caregivers. For example, researchers may share opportunities directly with healthcare providers or community organizations, but how that information is shared with potential participants is outside of the researchers' control. Providers, community organizations and even friends and families may choose to share information about study opportunities; further, they may choose to share their trusted opinions of the research or research organization, which may be positive or negative. Again, these decisions and opinions are outside the control of the research organization(s).

The second component, small numbers bargaining, refers to the power parties have over a contract (Williamson, 1975). This may include the number of organizations or customers available to the party, or the routines or expectations that are expected from the agreement. From the participants' perspective, a researcher's small numbers bargaining includes eligibility criteria and structural elements such as the time spent to participate and the distance traveled for study appointments. From the researcher's perspective, the participant's and study partner's small numbers bargaining includes their ability to refuse to participate in a study. 
The third component, bounded rationality, refers to imperfect information surrounding the members or transactions, including any cognitive or emotional barriers affecting a person's ability to understand that knowledge (Williamson, 1975). Bounded rationality includes perceptions of research or motivation to enroll (or not enroll) in a study. For example, although studies may be available, a person with ADRD or caregiver may or may not be aware of them. Alternatively, a person who is averse to taking medication may not be inclined to look for studies if they perceive them to all require taking a study drug.

Bounded rationality can lead to the last component, opportunism, or acting in selfinterest (Williamson, 1975). For ADRD research, potential participants may feel that researchers are taking advantage of their circumstances (having a diagnosis of ADRD) because they must be willing to engage in all study activities. Additionally, the person with ADRD's availability to participate is contingent on their caregiver's ability and motivation to participate, potentially leading to additional dependence upon the caregiver.

Transaction cost theory illustrates why many people with ADRD do not participate in research -- the transaction costs are often too high for people with ADRD and/or their caregivers. In some of these cases, the costs are beyond the control of both the research organization and the potential participants. In other cases, the people with ADRD and their caregivers have weighed the costs to be higher than the benefits of joining a study. Understanding the decisions of people with ADRD and study partners to join a study and participate in research is more fully described in the context of civic engagement. 


\section{Civic engagement}

A second framework, civic engagement, describes how ability and motivation affect civic engagement -- the notion that community members contribute individually and collectively in order to better their communities (Mettler, 2002). Common examples of such contributions include donating blood, participating in neighborhood associations, or voting. Similarly, many people participate in clinical research with the intent of helping others (Law et al., 2014; Anderson, Borfitz \& Getz, 2018). Although there are costs to participate in research, including the time spent on study activities and distance traveled, as well as the costs of the study intervention, the motivation to contribute to the common good may supersede these negative aspects and make the transaction costs more acceptable. Figure 5.1 depicts how the decision to be civically engaged is developed and how civic engagement is applied to ADRD clinical research participation.

Figure 5.1 Policy Feedback for Mass Publics: How Policy Affects Civic Engagement

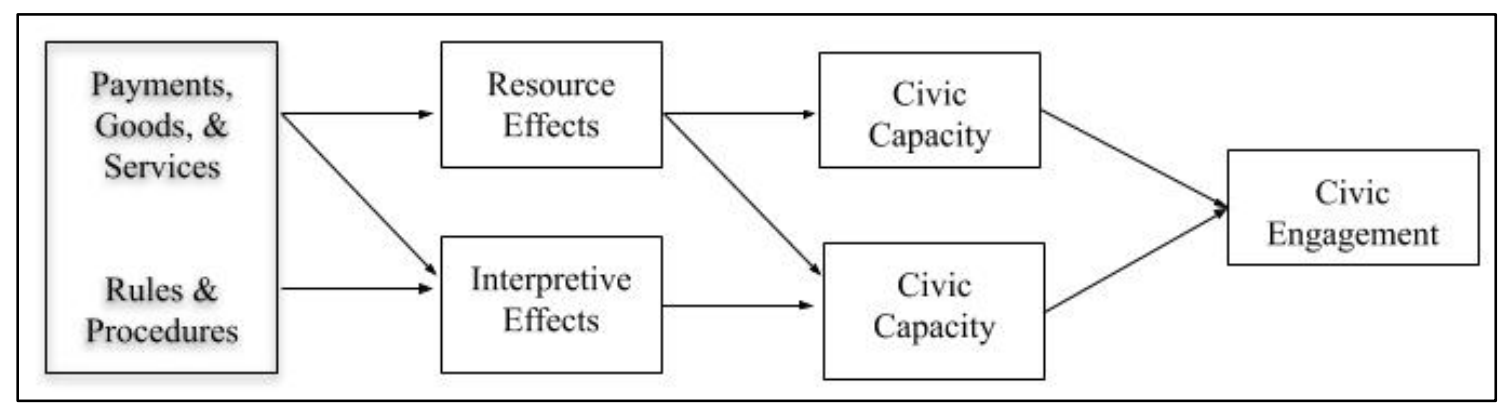

Source: Mettler \& Welch, 2004, p. 511

Mettler (2002) states that payments, goods, and services establish resource effects, defined as behaviors shaped by resources and incentives, which affect civic capacity, or the ability to be civically engaged. In the clinical research realm, these resource effects may include ease of transportation and receiving more information about 
studies (Karlawish et al., 2008; Golhoffer et al., 2015; Friedman et al., 2015, Mahon et al., 2016; Anderson et al., 2018). Resource effects also influence civic predisposition, defined as "the perceptions of their role in the community, their status in relation to other citizens and government, and the extent to which a policy has affected their lives" (Mettler, 2002, p. 352). In the clinical research contact, this can be interpreted as individual motivation to participate. Resource effects affecting civic predisposition may include altruism, financial incentives, and partnerships with trusted community leaders (Brown et al., 2013; Golhoffer et al., 2015; Cary et al., 2015; Friedman et al., 2015; Mahon et al., 2016; Black et al., 2017; Anderson et al., 2018).

Civic predisposition is also affected by the interpretive effects -- the information conveyed through policies (Mettler, 2002). Interpretive effects specific to ADRD research, including Congress's earmarked funding for ADRD research and NIA grants specific to increasing ADRD research participation, highlight that dementia research, and the need to recruit for dementia research, is important (Alzheimer's Association, 2019a; NIH, 2018). Interpretive effects also exist within the research organization. For example, if recruitment procedures are not inclusive of the needs of diverse groups, such as English language requirements, recruiting within a certain mile radius, or not giving presentations in areas that people of color frequent, they might convey messages that these communities are not important enough to recruit for studies, and may demotivate these groups of people. Ultimately, an individual's ability and motivation both affect their decision to participate. 
Various scholars have taken different approaches to encourage civic engagement in communities. Bellah and his colleagues (1991) suggest that a public commitment to building a) institutions, b) family support, c) quality education, and d) self-respecting work empowers individuals to participate and serve as community stakeholders. VigodaGadot and Cohen (2004) suggest moving away from the idea of viewing community members as subjects or voters; instead, the government (or in this context, the research organization) should treat community members as customers and be responsive to their needs. Denhardt \& Denhardt (2003) advocate for a public administration governing style that encourages civic engagement by including community members as active stakeholders.

ADRD research organizations can apply these approaches to civic engagement and adapt their own policies in order to be more responsive to their community's needs. In response to Bellah et al. (1991), several processes can indicate a public commitment to clinical research and empower people with ADRD and caregivers. First, organizations can share knowledge about research opportunities with people with ADRD and their caregivers. This could be directly through community presentations or listservs, or indirectly through providers' offices. They can also provide supports for people with ADRD and their caregivers by offering referrals to support groups, social workers, or community resources for memory-loss friendly activities. Research organizations can ensure quality education on clinical research history, purpose, and opportunities through presentations or vendor booths at community events. Lastly, they can recognize people 
with ADRD and research participants as respected, collaborative partners by including them on advisory boards or in the research design process.

When applying Vigoda-Gadot and Cohen's (2004) work on civic engagement to ADRD clinical research participation, this could translate to treating participants and study partners as customers and being responsive to their multiple and often complex health and social needs. For example, in order to increase participant enrollment, research organizations could introduce ways to reduce time spent in study visits and distance traveled to study appointments. Utilizing Denhardt and Denhardt's (2003) civic engagement work to illustrate ADRD clinical research participation, indicators of civic engagement for people with ADRD and caregivers as active stakeholders could be recognized through opportunities to serve on advisory boards or participate in listening sessions with researchers.

\section{Civic engagement and contingency theory}

In times of crisis, acts of civic engagement increase (Putnam, 2000; Sander \& Putnam, 2010). Given the propensity of the U.S. government, philanthropists, and dementia advocates to refer to ADRD as a public health crisis, one would expect acts of ADRD research participation to increase. Unfortunately, this has not been the case, possibly due to the factors described above (NIA, 2018).

However, civic engagement scholars have found that organizations, governments, and policies influence the community's ability and motivation to participate in civic engagement activities (Bellah, Madsen, Sullivan, Swidler, \& Tipton, 1991; Denhardt \& Denhardt, 2003; Vigoda-Gadot \& Cohen, 2004; Chandra et al., 2016). One could expect 
the same to be true for ADRD research participation as has been the case for cancer and HIV/AIDS, two other diseases for which the U.S. Congress has earmarked funding. However, the community driving forces that are behind these movements may not exist to the same level for ADRD. This can be due to a variety of factors, such as the stigma in being identified as a person with ADRD or a caregiver, the progressive cognitive decline associated with ADRD, and the low rates of ADRD researchers collaborating with people with ADRD and caregivers in the same way that researchers have learned to collaborate with people with cancer or HIV/AIDS (Hermann et al., 2018).

The U.S. government has taken measures to increase participation through earmarked funding for ADRD studies, a national report on improving research participation, and grant opportunities for researchers to develop strategies to increase research participation in their local communities (Alzheimer's Association 2019a; NIA, 2018; NIH, 2018). Research organizations can rely on contingency theory, or modifying rules and processes, in order to be responsive to their community's needs to the extent that organizational and external policies allow. Modifying these rules and processes may increase the ability and motivation of people with ADRD and caregivers to participate in ADRD clinical research (Lawrence \& Lorsch, 1967; Galbraith, 1973). Modifications can include extended clinic hours, study activities conducted over the phone or telemedicine, and/or partnering with communities of color to identify their needs and form a trusting relationship. Table 5.1 summarizes how each theory is applied in this study. The purpose of this study is described in the next section. 


\section{Table 5.1 Theory Summary}

\begin{tabular}{|l|l|}
\hline \multicolumn{1}{|c|}{ Theory } & How it Relates to ADRD Clinical Research Participation \\
\hline Transaction Cost Theory & Describes the costs of participating in a study \\
\hline Civic Engagement & $\begin{array}{l}\text { Describes how ability and motivation affect the decision to } \\
\text { participate in a study }\end{array}$ \\
\hline Contingency Theory & $\begin{array}{l}\text { Describes how, and to what extent, organizations can } \\
\text { address the needs of people with ADRD and caregivers }\end{array}$ \\
\hline
\end{tabular}

\section{Purpose of this study}

The purpose of this study is to identify barriers and facilitators to dementia research participation from the perspective of people with ADRD and their caregivers. In order to increase ADRD research participation, the NIA allocated funding for studies that focus on ADRD clinical research recruitment, retention, and diversity of race, ethnicity, gender, and socioeconomic status. Additionally, NIA released a national strategy for improving research participation in ADRD studies in October 2018 (NIA, 2018). The national strategy provides recommendations that address ADRD research participation. However, the national strategy does not include a comprehensive list of barriers and facilitators; the NIA recommended continued research on identifying more barriers and facilitators for ADRD research participation (NIA, 2018). Further, these recommendations were developed by working groups of ADRD clinicians, researchers, and advocates; the voice of individuals with ADRD was not included despite calls from academics, collaboratives, and advocates to do so (Iliffe, McGrath, \& Mitchell, 2013; Kremer, 2017; NIA, 2018). This study contributes to the field by identifying additional factors affecting ADRD clinical research through the incorporation of perspectives of people with dementia and caregivers as experts in the Portland metropolitan area. 


\section{Methodology}

\section{Study design}

The current study addresses one of the three aims that a larger dissertation research project investigates. Data reported here come from semi-structured interviews with 12 dyads of individuals $(n=24)$ living with mild cognitive impairment (MCI) or ADRD and their caregivers. Although MCI is not considered a form of ADRD, clinical research studies frequently include people with MCI, as MCI is hypothesized to progress to ADRD (Roberts and Knopman, 2013). Dyadic interviews were employed as they recognize and use the relationship between two people as a source of information (Caldwell, 2014). Furthermore, dyadic interviews are frequently used when interviewing people with cognitive impairment because they provide interviewees with more control of the interview situation, reduce the need to conform to preconceived notions of what the interviewer is looking for, and empower interviewees to participate actively (Morgan, 2008; Morgan et al., 2013). Both dyad members were given a $\$ 25$ gift card to the grocery store of their choice. Dyads were recruited through local memory cafes, early-stage memory loss and caregiver support groups, and research organizations. More detailed information about the interview procedures is described below. All protocols were reviewed and approved by the Portland State University Institutional Review Board (IRB \#184914).

In addition to interviewing 12 dyads, a community-engaged approach to the research enhanced study rigor, relevance, and reach, and identified community-specific 
factors and strategies (Balasz \& Morello-Frosch, 2013; NIA, 2018). This approach recognizes interviewees as experts in the field of living with MCI or ADRD, or caring for a family member with MCI or ADRD and including them in the development and involvement of a community advisory board (CAB) to advise and guide study processes. Further, establishing a $\mathrm{CAB}$ is considered a good practice in community-engaged work (Newman et al., 2011; Kremer, 2017). CAB recruitment was facilitated through the Oregon Alzheimer's Disease Research Center, the Alzheimer's Association, and the PreSERVE Coalition for Black/African American Memory and Brain Health. CAB membership was comprised of researchers, clinicians, advocates, individuals with $\mathrm{ADRD}$, current and former caregivers, older African Americans, and a member of the researcher's dissertation committee with experience working with CABs in communitybased research. The 12-member $\mathrm{CAB}$ met three times during the study to guide the investigator on recruitment and interview protocols, interpret findings, and develop dissemination strategies. CAB community members (people with ADRD, caregivers, and older African Americans) were given a grocery gift card for each meeting they attended or contributed to, in recognition of their contributions to the $\mathrm{CAB}$.

\section{Data collection and analysis}

Semi-structured interviews with dyads took place starting in August 2019 and concluding in December 2019. Dyads were eligible if they spoke fluent English and lived in, received care within, or participated in an ADRD clinical research study based in the Portland, OR metropolitan area. Additionally, people with MCI or ADRD needed to have a clinician-assessed diagnosis, were considered to be in the mild or moderate stages per 
provider visit within the last six months, and were able to discuss their perceived values of dementia clinical research. Individuals with MCI/ADRD who did not have a caregiver were ineligible for the study.

Dyad members were interviewed separately. Caregivers were interviewed first in order to obtain detailed information about symptom recognition and diagnosis; the caregivers were allowed to sit in the interview of the person living with MCI or ADRD on the conditions that they remained quiet and the person living with MCI or ADRD preferred having them present. Interviewees were asked about the first signs of ADRD or MCI and the diagnosis experience, their knowledge of clinical research, how and if they received information about research opportunities, their perceptions of clinical research, their decisions to enroll or not enroll in studies, and suggestions for improving ADRD clinical research enrollment. They were also asked to review a list of factors identified in earlier research (see Chapter 4) and identify their level of agreement with those factors. This empowered people with ADRD and caregivers by asking about their experiences and invited a power-sharing context by recognizing them as experts.

Interviews were audio-recorded, and the investigator took field notes during and after each interview. Interviews were de-identified and transcribed using transcription services (Rev.com), then uploaded to Atlas.ti 8.1 software for coding and thematic analysis (Scientific Software Development $\mathrm{GmbH}$ ). The codebook emerged from the interviews, but codes were informed by the investigator's a priori knowledge of the literature and various theories and framework. Codes included sentences or sentence fragments that represented data and concepts (Miles, Huberman, and Saldana, 2020). The 
coding process began by coding broad themes then coding with details. For example, if an interviewee noted they did not have time to participate in ADRD clinical studies described lack of awareness, it was coded as “ability, barrier, time.” Conversely, if an interviewee said they participated in research because they wanted to help others, it would be coded as "motivation, facilitator, altruism." Much of the analysis was deductive, meaning the codes were analyzed with the notion that they may align with orienting concepts derived from the literature and theories (Miles et al., 2020). An inductive approach was also used to code for themes that did not correspond with existing literature and theories to identify themes that emerged from the data organically and mitigate the possibility of coding those themes based on current literature rather than the interviews (Lapadat, 2010). The investigator was the single coder, and the CAB interpreted findings by identifying what was most important, surprising, or missing from the themes and subthemes.

\section{Results}

Semi-structured dyadic interviews were conducted with 12 dyads of individuals $(n=24)$ living with MCI or ADRD and their familial caregivers. Table 5.2 provides the characteristics of the interviewees. Six of the dyads had never participated in dementia research, five were currently participating in at least one study, and one had previously participated in clinical trials. Of the five people with ADRD currently participating in research, one was in a clinical drug infusion trial, two were in separate observational home-based studies that involved unobtrusive technologies to identify real-time brain and 
physical health changes, one was in both a clinical trial and a community-based technology study, and one was a participant in a longitudinal cognitive testing cohort study. Ten of the 12 caregivers were spouses (50\% female), and two were adult children (50\% female). Eight of the 12 people with ADRD were diagnosed with Alzheimer's, two were diagnosed with MCI, one was diagnosed with mixed dementia, and another was diagnosed with dementia with Lewy bodies.

Table 5.2 Characteristics of Interviewees with ADRD and Caregivers

\begin{tabular}{|l|l|l|l|c|}
\hline Gender & Caregiver & $\begin{array}{c}\text { Research } \\
\text { participation }\end{array}$ & $\begin{array}{c}\text { Most Recent } \\
\text { Diagnosis }\end{array}$ & $\begin{array}{c}\text { Years Since } \\
\text { Diagnosis }\end{array}$ \\
\hline Male & Wife & Yes & Mixed & 3 \\
\hline Male & Wife & No & Alzheimer's & 4 \\
\hline Female & Daughter & No & Alzheimer's & 5 \\
\hline Male & Wife & No & Alzheimer's & 5 \\
\hline Female & Son & No & Alzheimer's & 3 \\
\hline Female & Husband & No & Alzheimer's & 3 \\
\hline Female & Partner & No & $\begin{array}{l}\text { Dementia with } \\
\text { Lewy Body }\end{array}$ & 2 \\
\hline Female & Husband & Yes & MCI/Alzheimer's & 2 \\
\hline Female & Husband & Yes & MCI/Alzheimer's & 4 \\
\hline Female & Husband & Yes & Alzheimer's & 1 \\
\hline Male & Wife & Yes & Alzheimer's & 4 \\
\hline Male & Wife & Yes & Alzheimer's & 3 \\
\hline
\end{tabular}

The key findings are summarized in Table 5.3, organized into three themes and 12 subthemes. These are discussed below. 
Table 5.3 Themes and Subthemes from Interviews

\begin{tabular}{|l|l|}
\hline \multicolumn{1}{|c|}{ Themes } & \multicolumn{1}{c|}{ Subthemes } \\
\hline Symptom recognition and diagnosis & - Symptom recognition \\
& - Diagnosis \\
\hline $\begin{array}{l}\text { Factors affecting the ability to } \\
\text { participate in research }\end{array}$ & - Knowledge of opportunities \\
& - Ineligibility/withdrawal \\
& - Time/distance \\
\hline Factors affecting motivation to be & - Using the Internet \\
in research & - Helping others \\
& - Living good years to the fullest \\
& - Taking study drugs \\
& - Advisory boards \\
\hline
\end{tabular}

\section{Theme 1: Symptom recognition and diagnosis}

Lack of a formal diagnosis is described in the literature as a barrier to research participation and was discussed frequently in the interviews (Chin et al., 2011; Grill \& Galvin, 2014; Corriveau et al., 2017). Caregivers and people with ADRD described the diagnosis process in two parts: symptom recognition and receiving a diagnosis. These are the two subthemes discussed subsequently.

Symptom recognition: Several caregivers stated that when they or their family members noticed initial symptoms, such as forgetfulness or difficulties at work, the caregiver assumed these symptoms were due to stress or aging. Some caregivers mentioned an "aha" moment when they noticed their spouse or parent had difficulties with driving or not recognizing the time of day, which led them to make an appointment with their provider. One spousal caregiver noted that she thought something was out of the ordinary but questioned herself: 
Caregiver: "It's not like suddenly he started doing something he'd never done before, it's that stuff that he'd always done and every wife in the world complains her husband doesn't finish projects that he starts out on. It just went from being a four or five on a ten point scale to an eight or a nine. You're like, "Is it me? Am I just being way picky and hard to live with, or is there something?"

Several people with ADRD were able to identify a specific event or several incidents where they recognized symptoms, such as not being able to get things done at work. Those who recounted substantive signs of memory changes, such as trouble driving or being outside and not remembering where they were, also noted that they received a diagnosis relatively quickly after seeking help. Others with earlier symptoms, such as one person who had difficulties at work or trouble with their crossword puzzles, recalled feelings of dismissiveness from their providers. They also stated that it took years to get a diagnosis:

Person with ADRD: "I knew that there were some issues. And so I went to my family care physician and I said, "You know, I just can't remember things like I used to." And he just discounted it ... So then I went and spent another few years after that and it just kept continuing to get worse."

Diagnosis: Both people with MCI did not have a confirmed Alzheimer's disease diagnosis but believed they had progressed to Alzheimer's disease based on contextual clues, such as the need to have Alzheimer's to be accepted into a drug trial. Two of the dyads noted their diagnoses were questionable, but for different reasons:

Caregiver: "[The neurologist] said, "Well, since it doesn't look like it's getting any better, probably getting worse ... MRI didn't show anything," he says, "She has some kind of cognitive impairment." Basically, Alzheimer's [was written down] because he says cognitive impairment insurance companies can sometimes be jerks about... They won't pay."

Caregiver: "Dementia, conceivably Lewy body ... The underlying observations for Lewy body were really pretty flimsy. One time she self-reported a visual 
hallucination, but as our separate counselor said later, he wouldn't trust anyone's self-reporting of a hallucination."

Though they clinically exhibit MCI symptoms, two people with ADRD noted that they

received a diagnosis of Alzheimer's after receiving a PET scan. One expressed

skepticism of his diagnosis:

Person with ADRD: "After going there for two years and being tested, she was ready to tell me I don't need to come back anymore because "we don't think you have [Alzheimer's]. But we want to ... give you a PET scan." Well, the fellow that reads it couldn't see anything but [they said] the software picked it up. That doesn't sound like a real definite diagnosis."

Some dyads noted that receiving a diagnosis was somewhat positive because they could

finally explain the changes, and in one case, resulted in the ability to complete their

"bucket list" and focus on their creative abilities:

Person with ADRD: “Just because you have dementia doesn't mean you've lost your life. In fact, it's been a breakthrough for me ... Because now I do paintings and [I] would never have done that without [the diagnosis]."

Others described negative interactions with their providers:

Person with ADRD: "At first [the neurologist] was sort of dismissive, "Oh, I'm sure you're just being neurotic," kind of message ... he comes back, he throws down the papers on the table and he said, "You're right! You do have a problem!"... It made me want to laugh. It was so shocking. But I just kind of sat there in stunned silence. I just thought, "Wow. Do you let all of your patients know the results that way?"

Person with ADRD: "If you don't care anymore about your patient than [having the secretary call to give the diagnosis], he needs to be doing something else ... He was so lackadaisical, like, "Geez, I've done this test for so many times"... I don't know if I should [go see another neurologist]. I'm scared to go to one now."

Summary: Substantial symptoms resulted in immediate worry and concern for providers, and quite often for the caregiver. Less obvious symptoms were noticed more often by the 
person with ADRD who felt their symptoms were minimized or dismissed by providers, despite continued concerns, resulting in a delay in the diagnosis. In two cases, interviewees who received a PET scan received an early diagnosis of Alzheimer's although they had only exhibited MCI symptoms. For most of those cases, caregivers felt that they were able to recognize unusual behaviors as symptoms now that there was a diagnosis that they originally misattributed to stress or age, though at least one noted that she noticed changes before her husband did. Several interviewees noted unprofessional encounters with their provider that led to uncertain diagnoses or diagnoses. Aspects of transaction cost theory and civic engagement are evident in this theme. A lack of diagnosis is beyond the control of a research organization, but without it, those with undiagnosed ADRD are either ineligible or not actively looking for ADRD clinical research opportunities.

\section{Theme 2: Factors affecting ability to participate in research}

Both respondents who participated in ADRD clinical research and respondents that have not participated in research described similar factors affecting their ability to enroll in a study. These factors include knowledge of opportunities, ineligibility or forced withdrawal from a study, time spent and distance traveled to partake in the study, caregiver burden, and use of the Internet.

Knowledge of study opportunities: Knowledge of study opportunities varied in terms of awareness and avenue of knowledge. Those who participated in studies learned of them via brochures in their neurology clinic, a friend's referral, a referral through an Alzheimer's Association support group, and/or by doing their own online research. Of the 
two dyads that had participated in multiple studies, one was told of an additional study by their research coordinator, and another was told of a study by their neurologist. Some caregivers noted that they asked their clinician or support groups about opportunities, but either the providers/facilitators were unaware of any, or the caregivers were told to check their organization's website. Some noted that they had never heard of opportunities. In this particular study, four of the ten dyads directly asked the investigator if they could provide any information about research opportunities:

Caregiver: "I had asked our primary care physician. She did not recommend any particular person to contact ... Then we have a friend at church who was in a study at [research organization] ... and he knew I wanted to get [wife] in, so he gave me the contact person at [research organization]."

Person with ADRD: "It has never come up. [They] haven't asked me. You're the first one ... I don't know of any other research."

Caregiver: "In these smaller communities ... doctors don't automatically do referrals for research, for evaluations ... The doctor says, "Well, looks like you got dementia." That's it."

Person with ADRD: “I don't think it's come up. It's not that I'm opposed."

Several caregivers noted that they have heard of research opportunities but decided not to get in contact with the research coordinator for a variety of reasons. This is discussed later in this chapter.

Ineligibility/forced withdrawal: Three of the six people with MCI/ADRD who had participated in clinical research noted that they were ineligible for other studies or could not continue in the study. The first person was not eligible because he scored too high on cognitive tests. The second person was not eligible because of his age. The third person was withdrawn after exceeding the study's parameters post-intervention: 
Caregiver: "The first [study] was really sad one because [wife] got a drug ... and within a couple of days she just turned around and she was just sharp and full of energy, and it was wonderful ... And then, she tested out of the group ... Because they were only looking at a certain parameter, and she was above the parameter. So she tested out of the thing, and she ... [was] not happy when this was taken away from her. It really had been a help and her friends ... are saying, "Well, why can't they keep giving her that?"

The person with ADRD subsequently enrolled in two other trials, both of which were canceled by the study sponsor before the study ended. One trial was canceled due to a lack of positive results; neither the caregiver nor the person with ADRD could remember why the second trial was canceled.

Of the six dyads that had not participated in ADRD clinical research, none reported being told that they were ineligible; however, one speculated that they might have been eligible:

Caregiver: "I've put her name on a couple of different websites, where they're asking for research subjects, but nobody's ever contacted us. So, evidently, she didn't meet the criteria."

Time and distance: The time spent on research activities and driving distance to drive to attend these activities were major factors for many caregivers and people with ADRD. Most noted that driving to get to the various study sites affected their decision to participate in research:

Caregiver: "We drive out [across town] for an optometry appointment, and have to drive back, and it's just like that was what really put her off to it. And me, too ... I mean, they had one place for CT and one place for MRI ... [and then a third place for] optometry ... [Then], she had a hearing test at another place."

Caregiver: "It'd have to be convenient if we had to [drive]. [If it was in] southeast [Portland] at 9:00 in the morning and it took us two hours to get there, we would not engage in that one." 
One couple who drove two hours one way every four weeks to get to their study site had a different outlook on the time and distance they spent in the study:

Caregiver: "It's just something we plan on. Sometimes we just go up for the day, sometimes we meet friends and spend the night. It's a day, you spend three or four hours sitting in a chair. It's not particularly painful. Sometimes I take the dog with us, so I take the dog for a walk. We have a little routine, we listen to "Wait Wait ... Don't Tell Me" on the way up, or music. We have our favorite restaurants that we go to. So it's not difficult at all."

Caregiver burden: A few caregivers noted the demands of caregiving affect the ability

they have to participate in research:

Caregiver: "Well, it's just that my life is just so jammed full ... there's just so many things, that, I think, "Oh, why am I doing this? It takes time to fill out things. "I mean, if things get worse with [husband], I might have to just say, "I can't do it."

Caregiver: "Doing anything is a significant effort. It takes 20 minutes to get out of the house, you have to get it on the count. It's just a significant effort to do anything other than kind of hang around the house. ... [Husband] will go. He would just prefer not to."

Caregivers also noted how their responsibilities affect their ability to be in research, even

if they were not required to drive to study sites:

Caregiver: "Searching through a whole bunch of websites looking for research studies where the criteria might meet what she has, doing that is very timeconsuming ... She can't drive anymore. So, I've got to take her everywhere she goes, which I didn't used to do... And, I've got to do a lot of things with her and everything that [she] used to do independently and all. What that does is put the time-consuming burden on me."

Caregiver: "One I thought about doing ... it seemed very easy because we didn't have to go anywhere ... But the thought of using [the technologies], it just would have been a headache trying to explain, "Oh, this is for this, " constantly ... I'm already answering questions all the time, and then to add something else."

Using the Internet: Several caregivers noted that they did their own online research to

find providers and research opportunities. Their outcomes were mixed: 
Person with ADRD: "[Husband] gets online and he said, "Okay. As far as I can see, the best place on the West Coast to go is [research organization]." So I went down there and I got tested. And they confirmed, yes, I did have a problem. Then I was in a clinical trial down there."

Caregiver: "You get on these websites, and a lot of times they're in fairly scientific terms, which for the most part, yeah I can read and decipher. Most people can't ... To me, the ideal site would have to be a website that there'd have to be some other ways you can do it too in-person because not everybody is computer literate."

Caregiver: "One of the people at the [organization] said their website is a nightmare. She says ... it's a nightmare to navigate, so I doubt if I would have looked and found [the clinical trial] there."

Summary: There are a variety of factors affecting the decisions of a person with ADRD regarding participation in ADRD clinical research, including knowledge of opportunities, ineligibility or forced withdrawal from studies, time and distance, their caregiver's burden, and the ability to use the Internet to find research opportunities. In most cases, interviewees discussed these factors as barriers to research participation. Theories of transaction cost and civic engagement are prominently featured in these subthemes. Small numbers bargaining, bounded rationality, and resource effects affect a person's decision to participate in ADRD research.

\section{Theme 3: Factors affecting motivation to participate in research}

All interviewees believed that ADRD clinical research was essential and more people needed to participate in it; however, that did not necessarily mean they were willing to join a study. Factors derived from these interviews include the motivation to help others, living life to the fullest, taking study drugs, and advisory board participation. Motivation to help others: Nearly everyone recognized that a clinical trial would not be beneficial for themselves, although some noted that they hoped they would see a benefit 
if they participated. Rather, they participated or said they would consider participating to help others or give back in some way.

Caregiver: "People ask, "What's in it for me?" Sometimes, the answer is, "Well, they will find a cure and fix you." I think for Alzheimer's, that's not going to happen in her case. I think if I was diagnosed five years out, I don't know whether it would help me. But, it could help our kids."

Person with ADRD: “I didn't hesitate to get into clinical trials. What have I got to lose? And I like the feeling that even if it doesn't help me, it's contributing to knowledge. So for me, it's kind of giving back and hoping that maybe I'm getting some study drug; which I think I am."

Caregiver: "My thinking was, "This is so horrible. Something good should come out of it."

Person with ADRD: "I always felt it was my duty to do it. And so that's why I did it for as long as I did ... Because if I was suffering, I knew others were as well. And I really felt like this was a fight worth going into."

Caregiver: "If researchers could advertise that this is something that'll make you feel good because you're making a difference ... all of us want to be doing something I think."

For some, not receiving a direct therapeutic benefit was a sufficient reason to not

participate in available research opportunities:

Caregiver: "Well, to be honest with you, it was a couple of factors. One is that it's I don't think there's going to be anything that's going to reverse it... And I know that it could help people in the future .... But it's like, okay, do I want to add on one more thing, drive up to [research organization], which is like way the heck up there, and for what?"

Caregiver: "I don't think that there's anything to be done in our situation. We're working for someone else."

Living their life to the fullest: Several interviewees felt that having dementia was a race against the clock before the person with ADRD was no longer able to do what they enjoyed. Many believed they had limited time to live their lives to the fullest, and if they 
had to choose between participating in a study and doing things they enjoyed, they would choose the latter, as illustrated in the following quotes:

Person with ADRD: "I would prefer [a research study] not take so much time. I can't work on [my leisure projects] because I really feel that I don't have that much time [to work on those projects]."

Caregiver: "There are a lot of opportunities for us to do things that enrich what are still good years. If it was going to be some intervention that would interfere with that or gobble up a lot of our time, that would be a substantial negative."

Person with ADRD: "I participated in so many studies that after eight years I said, "Enough ... I want to have my life back."

Person with ADRD: "If there'd been a two-year lag period [to get into a research study], we would have continued to look for other options. As it was, I think we did wait for a few months, but with something like Alzheimer's, where you sort of feel little bits of your brain chipping off, you don't want to wait indefinitely."

Taking study drugs: When interviewees were asked how they would describe clinical research, most defined it in terms of clinical trials - testing an intervention to determine if it is effective for treating or preventing a disease. For five of the 12 caregivers and five of the 12 people with ADRD, taking a study drug was an automatic disincentive to joining a study, especially if there was no guarantee that the drug would be effective. Some interviewees alluded to having physical difficulty swallowing pills, while others noted concerns about safety, effectiveness, or side effects. Very few indicated that they would be willing to participate in studies where safety and efficacy have already been established, but still had concerns about side effects:

Caregiver: "I'm not big on any drugs so that rules out a fair amount of them."

Caregiver: "If the only research options were medication, I don't think that would [work] ... He has enough trouble taking [his] medications" 
Person with ADRD: "I think you can take one pill and it interferes with the results of the other pill, and I'm taking about six right now."

Person with ADRD: “Well, generally, I take some medications, and I will take more medications in the future. But I'm not for medication particularly ... unless somebody has advised me they are useful."

Caregiver: "[if a study coordinator tells us that] this drug has a potential for being really helpful, but in this stage of development, we know there are ... side effects that will probably happen, that would be a strong negative no ... We wouldn't [participate], we've got enough trouble already."

Caregiver support: Several caregivers felt unsupported, even if they were attending support groups. Although they acknowledged that while it was not the researcher's "role" to provide support, they noted that it could be a motivator for caregivers to join a study:

Caregiver: "They probably know a caregiver's under a lot of pressure. So ease some of that pressure and you might help with participation ... They could provide a caregiver and compensation for a couple of hours. They could have an activity that we could drop the person off at another time. They could help deal with any issues you're dealing with your person in particular. That's not their role, but those are useful things."

One caregiver noted that they report on their emotional health every week as part of their study participation and yearned for a response:

Caregiver: "You know, [my survey responses] are all going into a big file in a computer, and you think, "Here I am pouring out my heart about how lonely I was"... So, if I say I'm lonely, just lend me an ear, and give me wisdom about how to deal with this."

Advisory board participation: When asked about being on an advisory board where caregivers and people with ADRD collaborate with researchers and staff, most interviewees described them as unimportant to their decision to participate in research. Some caregivers mentioned wanting to participate in the future but were unaware of current opportunities. A small number of people with ADRD said they would be 
interested in serving on an advisory board. One interviewee was a former neurologist diagnosed with Alzheimer's, who speaks to medical students about treating individuals with dementia. He is also part of a research advisory board for the Alzheimer's Association, but was unable to join their advocacy board due to policies regarding caregivers:

Person with ADRD: "The Alzheimer's Association invited me to join an advocacy group; I said I'd be happy to do it but they required a partner because they didn't want people to travel on their own ... I don't know how much longer I'll be able to do that, but right now that's not an issue. But that was something that was etched in stone ... [so] they said how about a different division?"

Notably, several people with ADRD felt that they would not be able to contribute:

Person with ADRD: “My brain isn't doing very well. I don't think that's something that would help anybody with me getting confused. I don't need to help people when I can't [help] myself."

Person with ADRD: "I think I'm past being useful on an advisory board myself. I mean, I may have opinions that could be useful and people might be interested in. Fine. But ... I'm 80-whatever-it-is ... I feel I'm too old to be useful in that regard."

Summary: Helping others, living life to the fullest, caregiver supports, and taking medications were identified as factors affecting motivation to participate in research. Helping others and caregiver supports were listed as positive motivators whereas living life to the fullest and taking study drugs decreased motivation. While advisory boards were generally considered neither motivating nor demotivating for most interviewees, it is important to recognize that several people with ADRD felt that they had nothing to contribute to them because they felt they could not help anyone. Theories of transaction cost and civic engagement are both evident in these subthemes. In some cases, the cost of participating is too high because of the lack of direct benefit. In other cases, the 
interpretive effects of knowing that one has helped others were sufficient motivation to join a study.

\section{Integration of Findings and Theories}

These three themes, and the associated twelve subthemes, offer insights in understanding personal factors affecting the decisions of people with ADRD and their caregivers to participate in clinical research in their local community. Themes were similar across both dyads that have participated in ADRD research and dyads that have not, yet their decisions to participate in research were different. Civic engagement helps to explain the decisions of those who have participated in ADRD research, whereas transaction cost theory helps to explain the decisions of those who have not participated in ADRD clinical research. Contingency theory can be used to demonstrate how organizations can develop strategies to encourage people with ADRD and their caregivers to participate in research.

\section{Civic engagement and ADRD research participation}

Civic engagement can be used to describe people who participate in ADRD clinical research due to their beliefs that their participation is valuable to better their community. The interviewees who participated in research, and even those who are not participating but wanted to, recognize that their participation will not result in a therapeutic benefit to help themselves, but they are doing so to help friends, family, or future generations, or to contribute to science. For example, one dyad noted that though they drive two hours each way to be in their study, they believe time spent driving and 
the distance traveled is worth it in exchange for the benefit of helping others and potentially receiving a therapeutic benefit.

An application of Mettler \& Welch's (2004) framework can be used to understand factors increasing the likelihood of ADRD research participation, where civic capacity is viewed as "ability" and civic predisposition is viewed as "motivation." In these cases, factors identified by the interviewees that affect their capacity, or ability, to participate in research include knowledge of opportunities, eligibility criteria, time and distance required to participate, caregiver burden, and use of the Internet. Similarly, predisposition, or motivation, to participate in research includes the chance to help others, living good years to the fullest, caregiver support, taking study drugs, and joining advisory boards. These factors may also compete. A person might be motivated to participate in ADRD research, but if they would be required to take study drugs, their motivation may be negatively impacted and they would choose to not join the study.

\section{Transaction costs and ADRD research participation}

For many interviewees, the transaction costs of participating in research are too high. Specific factors are identified in Table 5.4. With regard to uncertainty, specifically factors that are outside of the research organization's control, many of the caregivers and people with ADRD noted the lengthy time to recognize symptoms as dementia or receive a diagnosis from their provider. Part of this delay is due to attributing ADRD symptoms to stress or age rather than cognitive impairment; however, in some cases when a person went to their provider with concerns of memory loss, these concerns were dismissed. This resulted in diagnoses occurring several years after symptom onset. 
Table 5.4: Interviewees Transaction Costs of ADRD Research Participation

\begin{tabular}{|l|ll|}
\hline \multicolumn{1}{|c|}{ Component } & \multicolumn{1}{|c|}{$\begin{array}{c}\text { Examples in ADRD Clinical Research } \\
\text { Participation Literature }\end{array}$} \\
\hline Uncertainty & - & Symptom recognition and diagnosis \\
\hline Small numbers & - & Time spent and distance traveled \\
bargaining & - & Eligibility criteria \\
\hline Bounded rationality & - Knowledge of study opportunities \\
& - Using the internet \\
& - Living life to the fullest \\
& - Opinion of research benefits (direct versus non- \\
& & direct benefits) \\
\hline Opportunism & - Caregiver burden \\
& - Study drugs \\
\hline
\end{tabular}

Small numbers bargaining, or the bargaining power that researchers, study sponsors, research organizations, people with ADRD, and caregivers bring to the transaction, was also evident in the findings. Interviewees frequently noted that in order to join a study, they needed to meet all requirements set up by the researchers or sponsors. These often included a dementia diagnosis, meeting all eligibility criteria, and commitment to time and travel. In many cases, people with ADRD and caregivers made it clear that they were not willing to adhere to those standards (and potential inconvenience) just to participate in a study.

Examples of bounded rationality were evident in the interviews. Many interviewees were unaware of study opportunities, even in cases when they had asked their doctor or signed up for dementia research registries. Some interviewees noted that in order to search for dementia research opportunities online, they needed a high level of scientific understanding as well as time to search for research or sign up for registries. These factors, in addition to having Internet literacy, made it difficult for many caregivers 
and people with ADRD to use the Internet as a means for finding study opportunities.

Further, all interviewees were aware that the likelihood of receiving a direct intervention for MCI or ADRD from study participation was almost guaranteed to be unlikely. In these cases, individual beliefs about the benefits of participation differed. For some, the fact that participation might lead to an intervention for future generations, or at least contribute to science, was worth the effort. For others, the substantial unlikelihood of a direct therapeutic benefit equated to no benefit. In some cases, caregivers and people with ADRD noted that they believed that they only had a few "good years" left before the disease progressed too far to fully enjoy life. In these cases, they decided not to participate in study opportunities because this could take away from their perceived limited time remaining to live their lives to the fullest while they still could.

Examples of opportunism were also present in the findings. For several caregivers and people with ADRD, research opportunities that required taking a study drug was an automatic deal-breaker due to their dislike of taking pills or concerns of safety, effectiveness, interactions with other medications, and side effects. Many caregivers also noted elements of opportunism when they said they had no or limited free time due to taking care of the person with ADRD. In those cases, the spouse or parent would not be able to participate in research because their caregiver was unable to do so.

\section{Using contingency theory to reduce transaction costs and promote civic engagement}

By applying contingency theory, research organizations and sponsors can address frequently discussed barriers to participation. Research organizations could collaborate with study sponsors to reduce the time spent and distance traveled for study activities. 
For instance, they could work with local healthcare providers to offer centralized locations for study activities, such as CT scans or hearing tests, or work with multiple site locations so participants could choose the site closest to them. They could also seek to provide more opportunities for in-home study participation or provide care services for those who do not want to, or cannot, drive to the research clinic or other study activity sites, such as working with home nurses to provide infusions instead of requiring an actual visit to the study site.

Not all of the people who did not participate in research described that the transaction costs were too high. In fact, there were several interviewees who said they had attempted to learn more about research opportunities but were not told of any studies, yet would happily participate if given the chance. Although the connection between hypothetical willingness and actual participation is not strong (Buchbinder et al., 2004; Burke, 2014; Grill \& Galvin 2014; Inungu et al., 2017), organizations could modify their rules or processes to increase the likelihood of turning ability and motivation into participation. If a person with ADRD or a caregiver is unaware of research opportunities, they are unable to participate in studies. In these cases, knowledge transfer could be used to inform communities about research studies.

Information from researchers is translated to communities, such as a trusted healthcare provider or a community organization, which requires determination of what information is important to the community, who is a credible and trusted messenger, how the message should be transmitted, and the effects the message should have (Lavis et al., 2003). For example, the Alzheimer's Association may be less likely to share information 
about a drug trial at their support groups, but more likely to share knowledge about a study that includes some sort of caregiver benefit. Additionally, ADRD researchers could refocus their recruitment efforts and focus on giving presentations in areas where people with ADRD and caregivers congregate. Researchers interviewed for Phase 1 of this study noted that they post flyers or give community presentations at senior centers. This may be an ideal recruitment location for healthy adults for dementia prevention studies, but not necessarily for the target population of those with ADRD. Researchers might instead focus on areas where people with ADRD and caregivers are likely to congregate, such as support groups, memory cafes, or respite and day centers.

Knowledge transfer can also be important for letting people know the differences between clinical research and clinical trials. Clinical research can encompass numerous observational studies, from cognitive assessments to neuroimaging to study of in-home technologies. Clinical trials could include study drugs, herbal supplements, diet, exercise, technology, or social engagement as the intervention. Further, potential study participants who are concerned about physically swallowing study drugs might be more inclined to participate in studies that involve infusions rather than pills. Those who are concerned about safety, effectiveness, or side effects may benefit from learning about the different clinical trial stages and may be more willing to participate in large-scale studies that test the effectiveness of a drug against the current standard treatment. Not all studies require study drugs, and potential participants could be better informed about non-drug alternative study opportunities. 
To address caregiver burden, which is a frequently cited reason for not participating in ADRD clinical research, research organizations and study sponsors could modify some of their processes to better address caregiver needs. Many of the caregivers noted that they would like some sort of support if they were participating, either in the form of respite or support when they are going through difficult times. According to these caregivers, organizations and sponsors could better respond to their needs and provide these resources. This study's findings also suggested that research organizations could modify how they articulate their view of caregivers. If the participation of a study partner is a requirement for participation in a study by a person with ADRD, then they are participants, with differences in their study roles and activities. Thus, research organizations may consider treating study partners as participants and potentially compensate them for their participation (including time lost to other activities, such as employment). To identify specific caregiver needs, researchers could conduct focus groups with study partners and caregivers to identify their unique needs.

\section{Discussion}

The reasons why people with ADRD choose to participate in clinical research are complex, as participation requires both the ability and the motivation to participate. These interviews provided a unique opportunity for 12 people living with ADRD and 12 caregivers to describe in detail the process of recognizing and being diagnosed with ADRD, and their decision to participate or not participate in an ADRD clinical research study. Transaction cost theory, civic engagement, and contingency theory were reviewed 
to explain factors related to ADRD clinical research participation. Transaction cost theory and civic engagement illustrated why people with ADRD and caregivers may not have the ability and/or motivation to participate in ADRD clinical research. Civic engagement also identified factors influencing the decision to participate. Contingency theory illustrated how research organizations can modify their processes and rules to address the needs of people with ADRD and caregivers.

This research has several findings. First, there is an identified lack of knowledge about opportunities in the local Portland area. Many interviewees had never heard of research opportunities. While this is discussed frequently in the literature, this was surprising as most of these interviewees were recruited for this study from organizations with rich knowledge of research opportunities. In cases where they had searched for study opportunities online, many interviewees noted that websites and registries were difficult and time-consuming to navigate and often required scientific and Internet literacy. Second, neither a person with ADRD nor their caregiver's perceived value of ADRD research may increase their likelihood of joining a study. All interviewees agreed that ADRD research was important and necessary, but there were many factors-including time and distance, not receiving a direct benefit, concern of taking study drugs, and caregiver burden -- that led to a decision to not participate in a study. This aligns with findings from the literature that articulates the lack of a relationship between hypothetical and actual willingness to be in a study (Buchbinder et al., 2004; Burke, 2014; Grill \& Galvin 2014; Inungu et al., 2017). 
Third, many caregivers either do not have the free time to join a study with their participants or do not want to give up their limited free time. However, several caregivers noted that they would take the time to participate in the study provided some sort of caregiver benefit to ease their burdens, such as support, education, and/or respite. The literature about ADRD study partners and caregivers describes caregiver support as a facilitator to their study participation (Karlawish et al., 2008; Cary et al., 2015; Black et al., 2017). Some organizations may be unable to provide supports because of a lack of resources or because of concerns that it may compromise the integrity of the trial. In these cases, coordinators can search for and recommend local caregiver supports.

Fourth, the number of people who avoid research because it involves taking study drugs was substantial among interviewees. However, there are many studies that do not require taking study drugs, including neuroimaging, dietary, exercise, social engagement and technology studies that investigate diet, or those that use technology in a person's home. Fifth, providers need more education on symptom recognition and professional diagnosis. Many people with ADRD and caregivers noted that symptoms were initially dismissed. Often, it was not until more substantial symptoms presented that further evaluations were conducted. Further, at least two people with ADRD noted unprofessional diagnoses from their provider, leading to one person stating that she is scared to see a neurologist again.

\section{Recommendations}

Several recommendations can be made based on this study's findings. First, researchers can partner with other healthcare and ADRD resource providers to share 
study opportunities. This may include sharing study flyers or brochures with providers or giving presentations at community organizations. Some interviewees in the first phase of this study noted the difficulty in recruiting participants from these community talks (see Chapter 4). More research may need to be conducted to identify why there is little response after presentations and what strategies can be developed to improve recruitment from these presentations. Knowledge of study opportunities could also be fostered by modifying both national and local online registries and clinical research websites. These sites and registries, including TrialMatch, ClinicalTrials.gov, and local research registries, offer a wealth of information about study opportunities but may be inaccessible to those who lack scientific or Internet expertise or are short of time for searches. Modifications may include using lay language, providing definitions to describe trials, and streamlining navigation and registration processes.

Second, researchers could advocate that study partners be treated as full participants, and seek sufficient funding to enable caregiver compensation for their time and effort. Though they engage in different study activities, such as answering questionnaires, driving to appointments, or ensuring the person with ADRD is adhering to study protocols, their participation is absolutely essential to the study. Further, their compensation should be provided in a manner that is most suitable to them as caregivers, such as caregiver education, respite, or as-needed support and advice, and also reflective of what the organization can feasibly provide. Researchers could budget for this compensation in grant proposals and/or seek out supplemental funding. 
Third, research organizations could develop strategies to remove or reduce the time spent and distance traveled in order to participate in research. For example, they could work with study sponsors to include more secondary sites for specialty testing (e.g. optometry or CT scans), or work with home health agencies to avoid traveling for some medical procedures.

Fourth, researchers could highlight the benefits of helping others. Many interviewees noted that they participated for the ultimate benefit of others, rather than themselves, and they derived happiness from that. Researchers could partner with people with ADRD and caregivers who participate in research to share the message of what it is like to participate in research and discuss the benefits of being a research participant. These messages can also include the differences among clinical research and clinical trials, study drug versus non-study drug clinical trials, and clinical trial phases.

\section{Limitations}

There were several limitations to this study. First, sampling bias is likely given that all but one of the interviewees were white individuals, and there were no African American participants despite targeted recruitment. This may be due to a variety of structural and systemic barriers. For example, one community advisory board member noted the hesitancy of African Americans to participate in clinical research given the egregious acts conducted by clinical researchers. Additionally, Oregon has a history of mistreatment of African Americans, racist policies and practices, and modern displacement of African Americans due to gentrification (Hannah-Jones, 2011; Parks, 2012; Multnomah County Health Department, 2014; Geiling, 2015). Because of these 
barriers, many older African Americans are hesitant to speak with an unknown white academic about their perspectives on clinical research. Additionally, the researcher had developed a relatively recent relationship with African American community organizations and had not yet established a trusting relationship.). As a result, these findings may or may not be relevant to African Americans with ADRD and their caregivers, and there may be other factors that were not explored. This limitation is further discussed in Chapter 7.

A second limitation is that these findings are specific to the Portland metropolitan area. However, saturation was reached through these interviews, meaning that new data was not adding new information to the findings (Miles et al., 2020). They may be contextually relevant for similar communities, but are not necessarily generalizable nor are they intended to be, in the quantitative sense. Rather, these findings can be transferable to other researchers, clinicians, or advocates in other metropolitans areas if they believe these findings may be applicable to their setting and specific context (Smith, 2017).

Third, there is always a risk of response bias, confirmation bias, and subconscious bias. Interviewees may have censored themselves due to the audio-recording of interviews and may have focused on what they perceived the interviewer wanted to hear, resulting in response bias. Confirmation bias is also likely given that the investigator was the single coder and drew upon relevant theories and literature for analysis. The investigator mitigated some of this bias by writing field notes immediately after each interview, making a conscious effort to self-assess when analyzing codes and themes, and 
engaging the $\mathrm{CAB}$ in member-checking and interpretation of analyses. Furthermore, while there are no conscious biases due to the researcher's identity, it is important to acknowledge that subconscious biases are possible due to her identity as an academic who is a proponent of ADRD research and community inclusion, and these may have led to response bias, where interviewees may have answered questions in a way that seems more agreeable to the investigator (Kovera, 2010; Kalu \& Bwalya, 2017). These biases are further explored in Chapter 7.

\section{Conclusion}

This research study identified personal factors affecting ADRD research participation in the Portland metropolitan area. These were identified through interviews with local people with ADRD and caregivers, some of whom have participated in clinical research. Identifying how these factors affect the decisions of a person with ADRD to participate in research is an important first step in increasing participation rates. Given these factors, strategies can be developed and implemented to improve the ability and motivation of people with ADRD to join research studies in the Portland metropolitan area. This study should be repeated in a larger context or as small pilot studies in other communities to determine contextual relevance and generalizability for other areas. Further, strategies implemented by researchers, research organizations, or study sponsors should be routinely evaluated to determine their feasibility and effectiveness. Researchers should be reminded to consider the larger picture -- finding preventive actions, 
treatments, or a cure for ADRD. Participation in clinical research is an important step towards achieving that goal. 


\section{CHAPTER 6: RECOMMENDATIONS TO IMPROVE LOCAL DEMENTIA CLINICAL RESEARCH PARTICIPATION}

\section{Introduction}

The United States Congress has allocated 2.8 billion dollars annually to find a prevention or treatment for Alzheimer's disease and related dementias (ADRD) by 2025

(Alzheimer's Association, 2019a). One critical factor in achieving this goal is recruiting enough people with ADRD and their caregivers who are willing and able to participate in clinical trials. Historically, ADRD clinical trial participation is low; only $10 \%-27 \%$ of people with ADRD are able to participate due to comorbidities, low cognition, and frailty (Grill \& Galvin, 2014; NIA, 2018). In addition, there are numerous policy, system, organizational, and personal factors affecting an individual's ability and motivation to participate.

For those who are able to participate, many factors affect their motivation to participate. One significant factor is the requirement of a study partner, typically a spouse or adult child, who can attend study appointments and ensure protocol adherence (RollinSillaire et al., 2013; Black, Taylor, Rabins, \& Karlawish, 2016). The necessity of a study partner requires that two participants need to be recruited and enrolled -- the person with ADRD and their caregiver partner (Karlawish et al., 2008; Watson, Ryan, Silverberg, Cahan, \& Bernard, 2014; Grill \& Galvin, 2014; Cary, Rubright, Grill, \& Karlawish. 2015; Black et al., 2016), unlike other clinical research where a study partner is not always needed. 
Many individuals affected by medical conditions, including ADRD, report an interest in joining relevant clinical research both for potential benefits for themselves and opportunities to help others (English, Lebovitz, \& Griffin, 2010; Law et al., 2014; Anderson, Borfitz, \& Getz, 2018). Despite these reports, clinical research participation rates are low (English et al., 2010; Law et al., 2014; Mahon et al., 2016; Anderson et al., 2018). One common sentiment from interested individuals is that they are unaware of how to seek or receive information about opportunities to join clinical research (Sood et al., 2009; Law et al., 2014; Anderson, Borfitz, \& Getz, 2018). Individuals often expect to hear about studies from their primary care provider and are more likely to participate when these providers inform them due to their trusting relationship. However, individuals may be more likely to hear of opportunities from media advertisements (Anderson et al., 2018).

Regulations intended to protect vulnerable populations may also restrict participation among people with ADRD. Potential study recruits with ADRD may be deemed ineligible for clinical trials due to comorbidities or contraindicated medications (Grill \& Galvin, 2014; Adams, Caffrey, \& McKevitt, 2015). As most people with ADRD are over age 65 and already treated for multiple comorbidities, recruitment is especially challenging (Rollin-Sillaire et al., 2013; Grill \& Galvin, 2014; Law et al., 2015, GilmoreBykovskyi et al., 2019).

Furthermore, investigators and study sponsors may require additional eligibility criteria to manage clinical and ethical risks associated with research on older adults with limited physical and cognitive function (Califf, 2006; Rollin-Sillaire et al., 2013). For 
example, despite more than one-third of all ADRD cases occurring in people aged 85 or older, just $8 \%$ of individuals participating in ADRD clinical research between 2000 and 2015 were over the age of 85 (Banzi, Camaioni, Tettamanti, Bertele, \& Lucca, 2016). Many studies place age limitations to individuals age 85 or younger due to concerns about complex health problems. Age restrictions pose a barrier to individuals who may be otherwise healthy and eligible to participate in clinical research, and further limit the pool of available participants. To address this barrier, the NIH recently reversed a policy (NOT-98-024) that allowed for arbitrary age exclusion; as of January 2019, clinical research, barring scientific or ethical reasons, can no longer exclude individuals based on age (NIH, 2017a). However, it is still unclear how strictly this policy has been interpreted and implemented. The NIH has invited public comments in preparation for an upcoming workgroup to evaluate and modify the strategy to improve recruitment and retention of underrepresented populations, including geriatric participants (NIH, 2019).

The NIA recently convened working groups to identify strategies to improve recruitment and retention in ADRD studies. Its report was released in 2018 and emphasizes the concept of local community collaboration, with four key strategies: (a) increase awareness and engagement; (b) build and improve research infrastructure; (c) engage local communities and support participants; and (d) develop an applied science of recruitment (NIA, 2018). The aforementioned factors affecting ADRD clinical research, and the literature in general, do not incorporate concepts of local community collaboration. More research is needed to examine how local community collaborative efforts might affect ADRD research participation. 


\section{Purpose of this study}

This study aimed to identify factors affecting local ADRD clinical research participation in the Portland, OR metropolitan area, and specifically focused on local recommendations for increasing ADRD clinical research participation. Roughly 67,000 Oregonians over the age of 65 are currently diagnosed with Alzheimer's disease, and this number is expected to increase to 84,000 by 2025 without an intervention to halt its increase (Alzheimer's Association, 2019b). This number represents about 9\% of adults 65 and older living in Oregon, slightly less than the national average of 10\% (United States Census Bureau, 2018; Alzheimer's Association, 2019). However, the actual numbers of those living with ADRD may be substantially higher because the figures cited do not include people under the age of 65 or those with non-Alzheimer's disease dementia. This number also does not include individuals who exhibit symptoms but are un- or misdiagnosed, which can be as high as $60 \%$ of those living with dementia in higher-income countries (Lang et al., 2017).

\section{Conceptual Framework}

The Culture of Health Action framework provides a useful structure to conceptualize recommendations to improve research participation among people with ADRD because many of the factors affecting clinical research participation can be categorized into the Culture of Health Action Framework's four action areas, illustrated in Figure 6.1. The Culture of Health Action Framework was introduced by the Robert Wood Johnson Foundation in 2014 and is designed to encourage collaboration across the health and 
social sectors with the goal of improving population health, wellbeing, and equity (Robert Wood Johnson Foundation, 2016). In the conceptualization of this framework, health providers partner with local nonprofits and private organizations. These partnerships then work with communities to define their needs and the measures that matter most to them to improve population health, wellbeing, and equity (Weil, 2016). Partners then design and redesign programs to better meet community needs and achieve the Culture of Health Action Framework's goals of improving community health, wellbeing, and equity (Weil, 2016). When compared with other communities, those with high-density multi-sector networks supporting health improvement activities have better health outcomes (McCullough \& Leider, 2016).

\section{Figure 6.1 Culture of Health Action Framework}

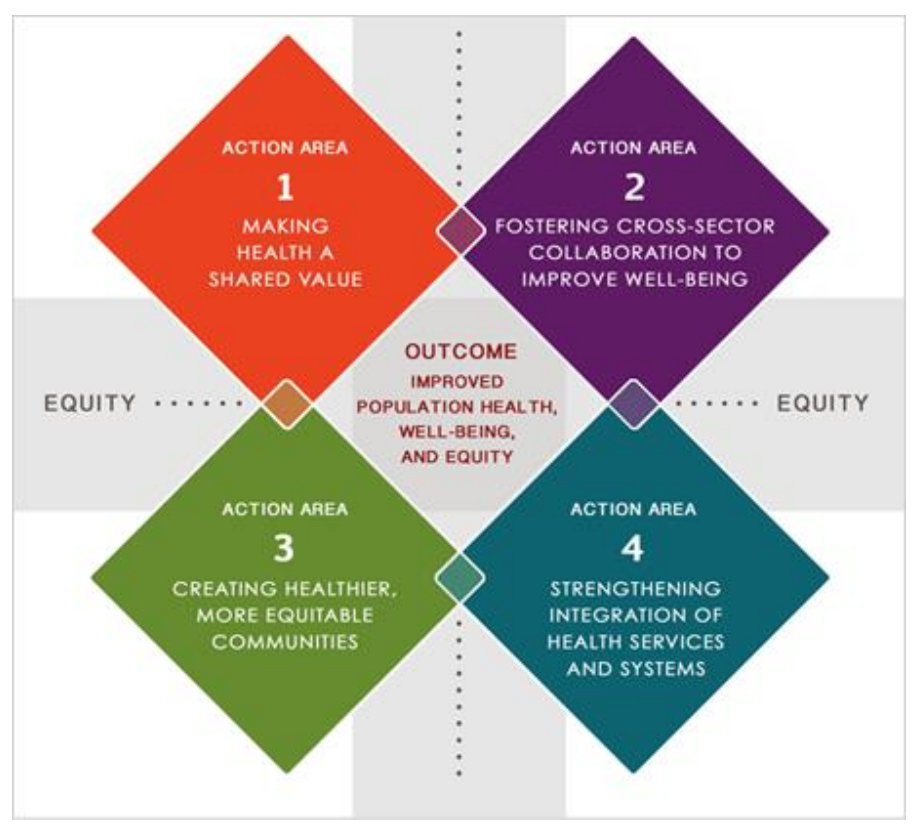

Source: The Robert Wood Johnson Foundation, 2016

There are several socioecological frameworks that may be used to articulate strategies to improve ADRD clinical research participation, but the Culture of Health 
Action Framework was chosen for a variety of reasons. The Culture of Health Action Framework and its action areas could be useful for articulating strategies to increase the ability and motivation to participate in ADRD clinical research. For example, the knowledge of opportunities from trusted providers and community leaders, and partnerships among community members, multiple organizations, and government, are strategies recommended by researchers to encourage motivation. This framework has not been applied to date in an ADRD context (based upon a literature review), but it could be applied to illustrate opportunities for people with ADRD. Traditionally, the voices of people with ADRD have not been incorporated in research design; however, this framework could be used to conceptualize an approach that would encourage the inclusion of people with ADRD in order to define their own needs and develop solutions to address structural barriers to participation. Although there is no strong evidence that people with ADRD identify themselves as part of an ADRD community, communityengaged principles can be used to include the voices of people with ADRD and their caregivers in research. Further, many of the factors that affect ADRD research participation can be contextualized using this framework and its four action areas, which are described in detail below.

\section{Culture of Health Action Framework Action Area 1: Make health a shared value}

The Robert Wood Johnson Foundation recommends three changes be made in order for communities to view health as a shared value. First, there must be a shift in mindsets and expectations of what individuals consider health and wellbeing, with an emphasis on group benefits and opportunities. Individuals must view health as shared and 
expected, not a function of economic privilege. Mindsets and expectations can shift by stressing the benefits of equitable health opportunities, but also require group-level empowerment in order to push for social change (Chandra et al., 2016). Second, health as a shared value requires a sense of community identity. Individuals and communities are interdependent by virtue of their sense of community membership (or lack thereof), belonging, and shared experiences. When community members recognize and act upon their solidarity, they are able to better mobilize and enhance their ability to thrive, be healthier, and drive change. Lastly, making health a shared value requires the knowledge of civic engagement skills, values, motivation, and opportunities within that community (Chandra et al., 2016).

In this context, this means that those in a position of power, including healthcare and service providers, researchers, advocates, and policymakers, must share those resources with community members. As noted above and in Chapters 4 and 5 of this dissertation, individuals with ADRD may want to participate in related clinical research but are unaware of how to seek or receive information about opportunities (English et al., 2010; Law et al., 2014; Mahon et al., 2016; Anderson et al., 2018). Many expect to hear about opportunities from their trusted provider or community leader but may be more likely to learn through media outlets (English, et al., 2010; Law et al., 2014; Mahon et al., 2016; Anderson et al., 2018). Knowledge transfer, where information from researchers is translated to communities, can be used to make ADRD research participation a shared value. Knowledge transfer requires determination of what information is important to the community, who a credible and trusted messenger would be, how the message should be 
transmitted, and the effects the message should have (Lavis, Robertson, Woodside, McLeod \& Abelson, 2003). Knowledge transfer can be used to directly solicit community members to participate, which increases motivation for research participation (Law et al., 2014).

\section{Culture of Health Action Framework Action Area 2: Foster cross-sector collaboration to improve wellbeing}

There are three unique drivers to foster cross-sector collaboration. First, highquality cross-sector partnerships result from having a sufficient number, variety, and quality of partners (Towe et al., 2016). Collaborations emerge from advocacy, community buy-in, and commitment from the partners (Towe et al., 2016). Second, partners must be informed of how they are expected to engage with and benefit from these collaborations, how the collaborations will address equity, and what will be the return on investment (Towe et al., 2016). Third, these collaborations can be developed through local, state, and/or federal level initiatives and policies across multiple agencies (Towe et al., 2016).

Cross-sector collaboration can be used to shape ADRD research and increase participation. The Satellite program, at Washington University in St. Louis, aimed to increase African American participation in dementia research. It illustrates how highquality cross-sector partnerships can lead to participatory processes and outcomes (Williams, Meisel, Williams, \& Morris, 2011). A federal grant from the NIA in 1992 provided the financial initiative to establish the program, and over several years, they developed partnerships with local clergy, the Alzheimer's Association, government 
offices, senior centers, libraries, and African American community organizations (Williams et al., 2011). Partnerships were sustained through numerous types of investments, including federal grants and philanthropic contributions (Williams et al., 2011). Policies were created to maintain their partnerships and achieve their goals, such as the establishment of an African American ADRD research advisory board in 2004 (Williams et al., 2011). Satellite achieved its primary goal of removing research participation inequity among the African American community in St. Louis; their success has led to similar programs being developed in California, Wisconsin, Kentucky, and New York (Williams et al., 2011; NIA, n.d.).

The NIH's May 2018 announcement of funding opportunities to promote diversity, recruitment, and retention through collaborative partnerships may serve as a gateway to knowledge mobilization in ADRD research (NIH, 2018b). Receiving an NIH grant is a financial and academic incentive for investigators, who may then implement programmatic changes within their research organization that establish or strengthen collaborative partnerships. They may also influence resource allocation and opportunities based on the expertise of the participants and study partners. Finally, NIH funding can also strengthen existing partnerships by providing funding to support collaborative activities.

\section{Culture of Health Action Framework Action Area 3: Create healthier, more equitable communities}

Improving physical environments, social and economic conditions, and policies that facilitate coalition-building and improvement projects will enhance opportunities for 
healthier and more equitable communities (Dubowitz et al., 2016). In order to achieve healthier, more equitable communities, the complexity of the causality of poor health outcomes must be recognized. Dubowitz and colleagues illustrate this challenge with the example of a grocery store: simply adding a grocery store in a neighborhood may improve the neighborhood's quality, but not improve healthy eating (Dubowitz et al., 2016). Individuals living in the neighborhood may prefer to purchase food from other locations, may not be able to afford the groceries, or may not have transportation to get to the store. Additionally, there may not be adequate structural supports, such as sidewalks or street lights, which help individuals to safely walk to the store.

New governance structures may need to be created to sustain these actions. Opportunities may exist when governmental departments work together (e.g. departments of health and transportation), but rely on elected and appointed executive officials who are supportive of these goals. Further, creating healthier and more equitable communities requires mobilizing broad coalitions amid political polarization (Dubowitz et al., 2016). When health sectors partner with community members as well as local government and organizations, stakeholders may begin to recognize the complex causality of health outcomes due to social determinants and individual choice and develop solutions that align with the community's needs.

The reasons why people with ADRD choose to participate in clinical research or not are complex, as participation requires both capacity and predisposition. Just as adding a new grocery store in a neighborhood does not automatically improve health outcomes, offering a new research opportunity does not necessarily improve recruitment and 
retention. As previously discussed in the clinical research section, multiple factors may impede or facilitate an individual's ability and motivation to participate in research. Collaboration among the research sector, people with ADRD, and other relevant stakeholders is the best way to identify barriers, facilitators, and solutions to increase participation (Grill \& Galvin, 2014; Mahon et al., 2016; Kramer, 2017).

The Culture of Health Action Framework also recognizes this necessity; stakeholders from health and other sectors work with the community to identify their needs and values and develop programs that align with them. Programs are designed and redesigned as needed, capacity, trust, and relationships among all stakeholders are strengthened as the partnerships share power, work together, and develop solutions. Furthermore, organizations often assume that individuals will enter a relationship if it is beneficial; however, if there is a history of bad relations or culture, the individuals will avoid the organization, as exemplified in the previous discussion on African American participation in clinical research. However, if the organization takes the time to establish a relationship to build trust and respect and provide full information about study opportunities in a clear, understandable manner, it may improve the likelihood that the person with ADRD and/or their caregiver will participate in the research (Danner et al., 2011, Frew et al., 2011; Grill \& Galvin, 2014; Mahon et al., 2016, Inungu et al., 2017). Culture of Health Action Framework Action Area 4: Strengthen health services and systems

Drivers that strengthen the integration of health services and systems include access, balance and integration, and consumer experience and quality (Martin et al., 
2016). Access to health services and systems requires reducing and/or removing barriers due to cost and insurance, language, transportation, office hours and culture. Balance refers to how resources are prioritized and allocated between and within the health and social sectors, whereas integration refers to the meaningful connections that are made between sectors (Martin et al., 2016). Integrating medical care, public health, and social services systems can connect community members to appropriate and timely information, supports, preventive services, and formal healthcare to improve health and wellbeing (Martin et al., 2016). This requires a focus on improving access to healthcare and removing barriers such as language, office hours, transportation, and culture. Removing these barriers may lead to patients' greater participation in their own healthcare because their ability to participate has increased (Gelmon, Bouranis, Sandberg, \& Petchel, 2018). Discussions on resource allocation and priorities, and their effects on experience and quality, are also necessary (Martin et al., 2016).

The literature on primary care medical home $(\mathrm{PCMH})$ offers examples of how health sectors can become more accessible, integrated and balanced, and improve consumer experience and quality, ultimately strengthening health services and systems. For example, in Oregon, where over $70 \%$ of the primary care clinics participate in the state's Patient Centered Primary Care Home program, most clinics provide office hours outside of the typical 9-5 workweek and all are required to have a 24/7 telephone line (Smith \& Merrithew, 2017; Gelmon et al., 2018). Clinic leaders report translating materials and hiring bilingual and/or multicultural staff to better serve their patient populations, and care coordinators build repositories of community resources or work 
with other local health- and social-sector organizations to address other structural barriers (Gelmon et al., 2018). Though there are no studies that have evaluated the PCPCH program from the patient and family perspectives to date, clinic leaders perceive a positive difference for their patients (Gelmon et al., 2018).

This patient-centered set of strategies could be adapted to working with people with ADRD. Research organizations could apply similar strategies for dementia clinical research participants and their study partners. Study visits and activities could be conducted outside of the typical 9-5 workweek, and materials, including neuropsychiatric tests, could be translated into various languages. Research coordinators could build repositories of community resources specific to dementia and caregiving needs, including memory cafes, caregiver education, support groups, respite, and long-term care services. Additionally, the NIH's Inclusion Across the Lifespan policy removes age as a barrier (NIH, 2017a). This increases the ability for older adults to participate in research because they are no longer arbitrarily excluded from ADRD clinical research studies due to their age, increasing potential recruitment of people who are older. Just as removing barriers and providing resources through the PCMH model may increase patient participation in their own healthcare because of increases in ability and motivation, the same could be done in ADRD research to be more patient-centered and improve the conditions and supports for research participation of people with ADRD and caregivers.

As described above, each of the Culture of Health Action Framework's four action areas may be used to illustrate recommendations to increase ADRD clinical research participation by addressing structural barriers and providing more inclusive 
opportunities for people with ADRD and caregivers to share their expertise and develop solutions to mitigate barriers and increase motivation to participate.

\section{Methodology}

The study discussed here addresses the third aim of the larger dissertation research project: Provide recommendations to research organizations, health clinics, and advocacy groups to facilitate ADRD clinical research recruitment and retention. The purpose of the dissertation project was to identify factors affecting the decisions of a person with ADRD regarding participation in clinical research. These recommendations are based on two prior aims: the first identified organizational, system, and policy level factors affecting participation in ADRD research studies, and the second identified personal factors affecting ADRD participation.

\section{Study Design}

For the first aim of the study, the investigator worked with several local health providers, researchers, community organizations, and a community advisory board $(\mathrm{CAB})$ to identify ADRD clinicians, researchers, and advocates to offer expert opinion on organizational, system and policy factors affecting ADRD clinical research participation. This study's data collection approach used semi-structured interviews. Interview questions were derived from the literature and various theoretical frameworks. Interviewees were ultimately selected based on organizational affiliation to ensure diversity in organizational representation. Potential clinicians, researchers, and advocates were recruited by email or phone. Phase 1 interviewees were not paid. 
For the second aim, which examined personal factors that affect research participation, the investigator conducted 12 semi-structured dyadic interviews (24 interviews total) with individuals living with mild cognitive impairment (MCI) or ADRD and their caregivers. Although MCI is not considered a form of ADRD, clinical research studies frequently include people with MCI given hypotheses about the progression of ADRD, the availability of new diagnostic technologies (such as PET scans) that can identify pathological symptoms of $\mathrm{AD}$ even if the person exhibits only clinical symptoms of MCI, and the knowledge that people with MCI are significantly more likely to progress to dementia (Roberts and Knopman, 2013; Rabinovici et al., 2019). Both dyad members were given a $\$ 25$ gift card to the grocery store of their choice. Dyads were recruited through local memory cafes, early-stage memory loss and caregiver support groups, and research organizations.

The investigator used elements of a community-engaged approach to enhance study rigor, relevance, and reach, as well as for its strength in identifying communityspecific factors and strategies (Balasz \& Morello-Frosch, 2013; NIA, 2018). This approach included recognizing the interviewees as experts in the field because of living with MCI or ADRD, or caring for a family member with MCI or ADRD, and including them in the development and involvement of a community advisory board (CAB) to advise and guide study processes. $\mathrm{CAB}$ recruitment was facilitated through the Oregon Alzheimer's Disease Research Center, the Alzheimer's Association, and the PreSERVE Coalition for Black/African American Memory and Brain Health. CAB membership was comprised of researchers, clinicians, advocates, individuals with ADRD, current and 
former caregivers, older African Americans, and an academic faculty with experience working with CABs.

The 12-member $\mathrm{CAB}$ met three times during the overall study to guide the investigator on recruitment and interview protocols, interpret findings, and develop dissemination strategies. CAB community members (people with ADRD, caregivers, and older African Americans) were given a grocery gift card for each meeting they attended or contributed to, in recognition of their work with the CAB.

\section{Data collection and analysis}

Semi-structured interviews $(\mathrm{n}=9)$ with clinicians, advocates, and researchers took place between April 2019 and July 2019. Interviewees were asked about their experience of working with people with ADRD, knowledge of clinical research and related policies, if and how they receive information about clinical research opportunities, dissemination of those opportunities, perceptions of organizational-, system-, and policy-level factors affecting clinical research recruitment and retention, and suggestions for improving ADRD clinical research enrollment.

Semi-structured interviews with dyads $(\mathrm{n}=12)$ took place between August 2019 and December 2019. Dyads were eligible if they spoke fluent English and lived in, received care within, or had participated in an ADRD clinical research study based in the Portland, OR metropolitan area. Additionally, people with MCI or ADRD needed to have a clinician-assessed diagnosis, were considered to be in the mild or moderate stages based on a visit with a provider within the last six months, and were able to discuss their perceived values of dementia clinical research. Dyad members were interviewed 
separately, with caregivers interviewed first, but caregivers were allowed to be present during the interview of the person living with MCI or ADRD on the condition that they remained quiet and if the person living with MCI or ADRD preferred having them present.

Interviewees were asked about the first signs of ADRD or MCI and the diagnosis experience, their knowledge of clinical research, how and if they received information about research opportunities, their perceptions of clinical research, their decisions to enroll or not enroll in studies, and their suggestions for improving ADRD clinical research enrollment. They were also asked to review a list of factors identified in Chapter 4 and identify their level of agreement with those factors. This empowered people with ADRD and caregivers by asking about their experiences and invited a power-sharing context by perceiving them as experts.

Interviews were audio-recorded, and the investigator took field notes during and after each interview. Interviews were transcribed and de-identified using a transcription service (Rev.com), then uploaded to Atlas.ti 8.1 software for coding and thematic analysis (Scientific Software Development GmbH, Berlin, Germany, n.d.). The codebook emerged from the interviews, but codes were informed by the investigator's a priori knowledge of the literature and various theories and framework, including the Culture of Health. Codes included sentences or sentence fragments that represented data and concepts (Miles, Huberman, and Saldana, 2020). The coding process began by coding broad themes then coding with details. For example, if an interviewee noted they were unaware of ADRD clinical research opportunities, it was coded as "ability, barrier, 
awareness." Conversely, if an interviewee said they participated in research because they wanted to help others, it would be coded as "motivation, facilitator, altruism." Much of the analysis was deductive, meaning the codes were analyzed with the notion that they would fit into a conceptual framework (Miles et al., 2020). An inductive approach was also used to code and analyze themes that did not correspond with existing literature and theories to identify themes that emerged from the data organically and mitigate the possibility of coding those themes based on current literature rather than the interviews (Lapadat, 2010). The investigator was the single coder, and the $\mathrm{CAB}$ interpreted findings by identifying what was most important, surprising, or missing from the themes and subthemes. All protocols were reviewed and approved by the Portland State University Institutional Review Board (IRB \#184914).

\section{Study population}

Thirty-three interviews with healthcare providers, researchers, advocates, people with MCI or ADRD, and caregivers were analyzed and included in this study. In Phase 1, nine healthcare providers, researchers, and advocates were interviewed. Interviewees were employed by or volunteered with several healthcare, research, or advocacy organizations of varying sizes and structures. In Phase 2, interviews were conducted with 12 dyads of individuals living with MCI or ADRD and their caregivers $(n=24)$. The characteristics of the interviewees are presented in Table 6.1. 
Table 6.1 Characteristics of Interviewees

\begin{tabular}{|l|c|c|c|}
\hline \multicolumn{1}{|c|}{$\begin{array}{c}\text { Category of } \\
\text { Interviewee }\end{array}$} & Number & $\begin{array}{c}\text { Previous participation } \\
\text { in research (ADRD } \\
\text { only) }\end{array}$ & $\begin{array}{c}\text { Gender } \\
(\% \text { female) }\end{array}$ \\
\hline Researchers & $4^{*}$ & $\mathrm{n} / \mathrm{a}$ & $75 \%$ \\
\hline Healthcare providers & $4^{*}$ & $\mathrm{n} / \mathrm{a}$ & $75 \%$ \\
\hline Advocates & 2 & $\mathrm{n} / \mathrm{a}$ & $100 \%$ \\
\hline Caregivers & 12 & $50 \%(\mathrm{n}=6)$ & $50 \%$ \\
\hline $\begin{array}{l}\text { People with } \\
\text { MCI/ADRD }\end{array}$ & 12 & $\begin{array}{c}50 \% \text { as study partner } \\
(\mathrm{n}=6)\end{array}$ & $58.3 \%$ \\
\hline
\end{tabular}

*One interviewee identified as both a researcher and a healthcare provider

Six of the people with ADRD had never participated in ADRD research, and five were currently participating in at least one study. One was in a clinical drug trial, and two were participating in separate observational home-based studies that involved unobtrusive technologies that identify real-time changes. One was in both a clinical drug trial and a community-based technology study. Lastly, one was a participant in a longitudinal cognitive testing cohort study. The one person with ADRD who was not currently participating in a study had previously been in clinical drug trials. Ten of the 12 caregivers were spouses and two were adult children. Eight of the 12 people with ADRD were diagnosed with Alzheimer's disease, two were diagnosed with MCI/Alzheimer's, one was diagnosed with mixed dementia (vascular dementia and Alzheimer's disease), and one was diagnosed with dementia with Lewy bodies. Table 6.2 summarizes the characteristics of the interviewees with ADRD. 
Table 6.2 Characteristics of Interviewees with ADRD and Caregivers

\begin{tabular}{|l|l|l|l|c|}
\hline Gender & Caregiver & $\begin{array}{c}\text { Research } \\
\text { participation }\end{array}$ & \multicolumn{1}{|c|}{$\begin{array}{c}\text { Most Recent } \\
\text { Diagnosis }\end{array}$} & $\begin{array}{c}\text { Years Since } \\
\text { Diagnosis }\end{array}$ \\
\hline Male & Wife & Yes & Mixed & 3 \\
\hline Male & Wife & No & Alzheimer's & 4 \\
\hline Female & Daughter & No & Alzheimer's & 5 \\
\hline Male & Wife & No & Alzheimer's & 5 \\
\hline Female & Son & No & Alzheimer's & 3 \\
\hline Female & Husband & No & Alzheimer's & 3 \\
\hline Female & Partner & No & $\begin{array}{l}\text { Dementia with Lewy } \\
\text { Body }\end{array}$ & 2 \\
\hline Female & Husband & Yes & MCI/Alzheimer's & 2 \\
\hline Female & Husband & Yes & MCI/Alzheimer's & 4 \\
\hline Female & Husband & Yes & Alzheimer's & 1 \\
\hline Male & Wife & Yes & Alzheimer's & 4 \\
\hline Male & Wife & Yes & Alzheimer's & 3 \\
\hline
\end{tabular}

\section{Recommendations and Discussion}

Based on the analysis of the quotes from these two phases of interviews, supplemented by the CAB's review and complemented from the literature, six recommendations are made to improve local ADRD clinical research participation: 1) identify and promote local champions for ADRD clinical research participation; 2) promote policies and processes that incentivize cross-sector collaboration; 3) recognize caregivers as full participants; 4) include people with ADRD and caregivers in the research design process; 5) offer alternative options to reduce participation burden; and 6) evaluate and improve relationships between providers/researchers and patients/participants. These six recommendations can be categorized according to the four action areas in the Culture of Health framework and are outlined in Table 6.3. 
Table 6.3 Culture of Health Action Areas and Corresponding Recommendations

\begin{tabular}{|l|l|}
\hline \multicolumn{1}{|c|}{ Action Areas } & \multicolumn{1}{c|}{ Recommendations } \\
\hline $\begin{array}{l}\text { 1. Making Health a Shared } \\
\text { Value }\end{array}$ & $\begin{array}{l}\text { 1. Identify and promote local champions for ADRD } \\
\text { clinical research participation }\end{array}$ \\
\hline $\begin{array}{l}\text { 2. Foster Cross-Sector } \\
\text { Collaboration to Improve } \\
\text { Well-Being }\end{array}$ & $\begin{array}{l}\text { 2. Promote policies and processes that incentivize cross- } \\
\text { sector collaboration }\end{array}$ \\
\hline $\begin{array}{l}\text { 3. Create Healthier, More } \\
\text { Equitable Communities }\end{array}$ & $\begin{array}{l}\text { 3. Recognize caregivers as full participants in the research } \\
\text { process } \\
\text { 4. Include people with ADRD and caregivers in the } \\
\text { research design process }\end{array}$ \\
\hline $\begin{array}{l}\text { 4. Strengthening Integration } \\
\text { of Health Services and }\end{array}$ & $\begin{array}{l}\text { 5. Offer alternative options in study design to reduce } \\
\text { participation burden } \\
\text { 6. Evaluate and improve relationships between } \\
\text { providers/researchers and patients/participants }\end{array}$ \\
\hline
\end{tabular}

The following sections are presented by the Culture of Health Action Framework action area and include quotes from the interviews and synthesis from the literature that integrate the findings and relate them to the recommendations.

\section{Culture of Health Action Framework Action Area 1: Making health a shared value}

Individuals are more likely to participate when they receive information from a trusted source (Lavis et al., 2003). Although there may not be a direct therapeutic benefit, people with ADRD and caregivers can discuss the benefits they have received from ADRD research participation, including altruism, contributing to the knowledge base, and potentially helping future generations. Further, information from trusted researchers, clinicians, and community members who have participated in ADRD clinical research may increase motivation to participate (Lavis et al., 2003; Grill \& Galvin, 2014).

\section{Recommendation \#1: Identify and promote local champions for ADRD clinical}

research participation: This recommendation is grounded in experience in recent years 
during which time the visibility of high-profile champions for ADRD awareness and funding has increased. In support of his mother-in-law, actor Seth Rogan co-founded a nonprofit, Hilarity for Charity, and testified to Congress about the necessity of funding ADRD research, bringing awareness to younger generations and politicians alike (Alzheimer's Disease Research, 2014; Hilarity for Charity, 2019). NCAA basketball coaching legend Pat Summitt founded the Pat Summitt Foundation before passing away from younger-onset ADRD in 2016. Her legacy spurred a new ADRD research clinic in Knoxville, TN, and annual awareness and fundraising events at college basketball games during "We Back Pat" week (The Associated Press, 2016; SEC Staff, 2019). Philanthropist Bill Gates, whose father has Alzheimer's disease, had led missions to increase ADRD research through a 50 million dollar donation, convening working groups, writing blogs about the need for people to participate in ADRD clinical research, and creating his "Part the Cloud" initiative with the Alzheimer's Association (Gates, 2019).

These high-profile champions help demonstrate the prestige champions have in the community and the recognition they bring to an issue. Similar to how national champions can spur ADRD research funding, awareness, and participation, community members can champion these causes at the local level. This may include people with ADRD and caregivers who participate in clinical research, clinicians, researchers, and advocates: 
Advocate: "We need to hear about it more. I mean, if I don't know about it ... If I had some pamphlets or if [researchers] want to come and I introduce you and say this is what you're doing, and if anybody wants to talk to you, meet you after ... I think it needs to be more of a priority. But like I said, I've never had any information on it."

Caregiver: "In these smaller communities ... doctors don't automatically do referrals for research, for evaluations ... The doctor says, "Well, looks like you got dementia." That's it ... if researchers could advertise that this is something that'll make you feel good because you're making a difference ... all of us want to be doing something I think."

Efforts to champion research participation could include community events or presentations where individuals with ADRD or caregivers describe their reasons for participating. Many interviewees noted that they participated for the ultimate benefit of others, rather than themselves, and they derived happiness from that. Researchers could partner with people with ADRD and caregivers who participate in research to share the message of what it is like to participate in research and discuss the benefits of being a research participant:

Person with ADRD: "I didn't hesitate to get into clinical trials. What have I got to lose? And I like the feeling that even if it doesn't help me, it's contributing to knowledge. So for me, it's kind of giving back and hoping that maybe I'm getting some study drug; which I think I am."

Person with ADRD: "I always felt it was my duty to do it ... Because if I was suffering, I knew others were as well. And I really felt like this was a fight worth going into."

Caregiver: "People ask, "What's in it for me?" Sometimes, the answer is, "Well, they will find a cure and fix you." I think for Alzheimer's, that's not going to happen ... But, it could help our kids."

Conclusion: In order to make health, or (in this context of this study) ADRD research, a shared value, there needs to be a shift in mindsets and expectations, level of civic engagement, and a sense of community (Robert Wood Johnson Foundation, 2016; 
Chandra et al., 2016). Identifying and promoting local champions for ADRD research can influence these three drivers. Interviewees described how they need more awareness of local studies to share more information and resources. Interviewees also described the importance of having local champions, especially people with ADRD and caregivers who participate in research, describe to others why they decided to participate and encourage community members to do the same.

\section{Culture of Health Action Framework Action Area 2: Foster cross-sector collaboration to improve well-being}

Core values of this action area of the Culture of Health Action Framework include the promotion of policies and investments that support collaboration, variety in the number, breadth, and quality of partnerships, and knowledge of what to expect from these partnerships (Towe et al., 2016). This action area encourages both internal and external changes at the local and state levels, such as removing a policy that forces a provider to use personal time for collaboration activities, as well as those that discourage conversations with patients about study opportunities, which may usher in partnerships with research organizations to learn about new opportunities for patients. The NIA's R24 funding opportunity, Examining Diversity, Recruitment and Retention in Aging Projects, is an example of a federal-level investment that intends to foster cross-sector collaboration to improve ADRD clinical research participation (NIH, 2018).

Collaborations may involve partnerships that focus on research design and event planning to reach shared and organizational missions. They could be less formal, where researchers partner with numerous healthcare and ADRD resource providers to share 
study opportunities or to share community resources with participants. This may include distributing study flyers or brochures to providers or giving presentations on healthy aging and dementia at community organizations. Investigators from research organizations can use this funding to establish cross-sector collaborations with local community organizations, including cultural centers or religious groups, following the example of the Satellite program at Washington University (Williams et al., 2011).

Additionally, funding could be used to reimburse providers and researchers who must use their personal time when engaging in community-based collaboration.

\section{Recommendation \#2: Promote policies and processes that incentivize cross-sector}

collaboration: In order to promote awareness and interest in study opportunities, collaborative efforts between organizations must take place across sectors within communities. In some organizations, efforts are blocked by policies forbidding discussions or interactions with patients about research opportunities:

Healthcare provider: "Because of the population that we treat, [the Centers for Medicare and Medicaid Services] has it in our regulations that we are not allowed to enroll our participants in research ... And it's really because we're a capitated plan ... we control nearly everything about our participants. So, if we don't approve it, and we don't transport them to it, and we don't provide it, they don't get it."3

Healthcare provider: "I suppose we could say, 'There are some research projects over at [research organization]. If you're interested you can contact this person or this phone number.' But that's on the edge of what we're supposed to do."

\footnotetext{
${ }^{3}$ Interviewee sees patients through the Program for All-Inclusive Care of the Elderly (PACE), a capitated comprehensive health and long-term service program for dual-eligible, frail, community-dwelling, older adults. (Centers for Medicare and Medicaid Services, 2011)
} 
In other cases, providers may be unaware of opportunities, resulting in patients or caregivers conducting their own searches for research opportunities, or simply remaining unaware:

Caregiver: "I had asked our primary care physician. She did not recommend any particular person to contact ... Then we have a friend at church who was in a study at [research organization] ... and he knew I wanted to get [wife] in, so he gave me the contact person at [research organization]."

Person with ADRD: "It has never come up. [They] haven't asked me. You're the first one ... I don't know of any other research."

These interviews highlight that there are organizational policies hindering conversations about research, which results in a lack of sharing knowledge of research opportunities. Interviewees also spoke of policies that have resulted in the avoidance of cross-sector collaboration:

Healthcare provider: "I honestly devoted hours and hours and hours to trying to develop processes and education. But, over time, it's been hard to remain enthusiastic because any time I spend working on things like that, comes out of my own personal time. And people don't seem to understand that I'm not funded to attend two to three hour-long meetings. And if I spend time in a meeting, then I have to make up for that time at the end of the day later."

Researcher: "It is a challenge, particularly for minority research, where the key element to successful research in minority communities is establishing a long standing relationship. In order to do that, it takes resources. You can't really go out and fund a grant to create those relationships. It's basically volunteer time in a way ... to develop those relationships."

Policies can be put in place to promote cross-sector collaboration, such as funding to develop these relationships, and funding for providers, researches, or advocates to partner with other organizations. Some of these opportunities exist, such as the NIA's R24 grant opportunity (NIH, 2018), so more work may need to be done to highlight these opportunities. Additionally, policies that aim to develop community collaborations and 
partnerships can be redesigned to ensure that goals are being met. For example, one researcher described an NIH policy designed to streamline research processes during multi-site collaboration by allowing one central IRB to approve study protocols for all of the sites. According to this researcher, the policy has not achieved its intended outcome:

Researcher: "I think institutions tend to stay within their bounds of what they're used to doing. One area this has clearly shown its face is in the area of trying to enact a uniform, a single consent process, so I won't have 20 different IRBs reviewing the same consent but coming up with 20 different versions ... It's been aspirational. It's still not happening. I still see just in recent experience with multi-center projects that we're supposed to use a central IRB or a single IRB. In practice, it still hasn't happened."

Many interviewees described how collaborations provide benefits to their organization

and to their patients or participants:

Researcher: PreSERVE [Coalition, comprised of researchers, African American community members, and other community organization representatives] tries to have a balance between asking people to participate in research, but not having it be like 'We're really just having this event because we need to have you participate in research.' We really try to communicate why this research is important to the community, what is the giveback, how is this a sustained relationship instead of a one-time, 'We get from you what we need."'

This researcher further described how one community organization provides benefits that are not research-related:

Researcher: "[The Alzheimer's Association] has tables at [our] events ... they have these materials about ... what are 10 signs of Alzheimer's disease? What are some free online workshops that you can attend? Where are there caregiving classes or even just bringing the person you're caring for even to the Alzheimer's Association to the Portland branch and they can do art activities while you discuss with other caregivers? So all those types of things really feel like it's for the community."

Other interviewees described partnerships that were not necessarily collaborations, but referral-based: 
Healthcare provider: "When we have a patient come in, and they have dementia or depression, our team works very closely with community resources, [for example] finding them a clinical psychologist for counseling or finding an adult daycare program where somebody with early dementia, a family member needs to go to work but needs to have them be in a safe place during the day."

Other researchers described new collaborations and partnerships -- one to improve research awareness and education in the local community and another to facilitate backend processes to reduce time delays:

Researcher: "the Oregon Alliance for Clinical Research, it's made up of a few different sites throughout Portland ... [and] is aimed at educating the population [about] what clinical research is and how it can help some individuals, especially if there's no other avenue for them to take."

Researcher: "The Alzheimer's Clinical Trial Consortium, ACTC, they help clinical trials get started up a little bit faster ... for example, having a master agreement between our institution and that sponsor so that we don't have to negotiate all of the parts of the contracts every single time."

Conclusion: Action Area 2 conceptualizes how partnerships and collaborations, and the policies that fund and support them, can predispose to equity and further inclusion of community members (Towe et al., 2016). Findings from these interviews demonstrate how collaborations can be used to promote equity and participation. They also highlight how a lack of communication or collaboration reduces knowledge of opportunities within the community. Finally, these findings highlight how organizational and government policies can be used to create and remove barriers.

\section{Culture of Health Action Framework Action Area 3: Creating healthier, more equitable communities}

This action area articulates how policies and processes may not have the intended outcome unless community input is included from the beginning. In many cases, this 
action area is applied to considerations of equitable communities through the built environment, social and economic development and policies (Dubowitz et al., 2016). In the context of this research, this action area is applied through a lens that equity may be better achieved through the inclusion of community members with lived experiences of living with or caring for someone with ADRD. Community members have the expertise that can only be developed from living in the community, which researchers, advocates, and clinicians may not share. Community members should be included to achieve desired results - in this case, improving ADRD clinical research recruitment. Collaboration and equity can be encouraged by recognizing caregivers as full participants, and by including both caregivers and people with ADRD in research design processes. This leads to empowerment and motivation to create more palatable options and ideas around research recruitment and participation. Further, removing power dynamics by including people with ADRD and caregivers in the design process and valuing the expertise of people with ADRD reduces inequities, which may lead to identifying problems and solutions that are needed to reduce inequities, remove barriers, and improve ADRD clinical research participation.

Recommendation \#3: Recognize caregivers as full participants: Although caregivers engage in different study activities from the person with ADRD, such as answering questionnaires, driving to appointments, or ensuring the person with ADRD is adhering to study protocols, their participation is absolutely essential to the study, as noted by some researchers:

Researcher: "Sometimes the participant is really gung-ho and ready and wants to be involved and the caregiver just doesn't care, so that's really unfortunate and 
we see that often enough, where it's the caregiver dropping the ball, just not following through, not getting back to us for scheduled appointments, and just lost through follow up, basically, which is really unfortunate."

When asked to provide recommendations to improve ADRD research participation, interviewees often focused on improving the caregiver's motivation to participate in research through education or caregiver support:

Healthcare provider: "If caregivers could get support ... sometimes maybe there's a small stipend or agreement that people get from research participation. But sometimes it could be in terms of supporting caregivers. They might see this as a plus instead of just a monetary part of it."

Caregiver: "They probably know a caregiver's under a lot of pressure. So ease some of that pressure and you might help with participation ... They could provide a caregiver compensation for a couple of hours. They could have an activity that we could drop the person off at another time. They could help deal with any issues you're dealing with your person in particular. That's not their role, but those are useful things."

Caregiver: "You know, [my survey responses] are all going into a big file in a computer, and you think, 'Here I am pouring out my heart about how lonely I was' ... So, if I say I'm lonely, just lend me an ear, and give me wisdom about how to deal with this."

As such, researchers should recognize study partners as full participants who deserve compensation for their time and effort. Caregiver compensation should be provided in a manner that is most suitable to them as caregivers, including education, respite, or asneeded support and advice. Researchers can include funding for caregiver support staff, such as care coordinators or social workers, within their grants, or simultaneously involve them in studies that focus on caregiver health and support. This specific strategy may prove problematic if research organizations do not have the capacity, or there is concern about the integrity of a clinical trial if the study partner is receiving their own intervention to address caregiver burden. In those cases, the organization can provide 
information about local resources (support groups and training programs, respite care, care coordinators or social workers who are trained to provide resources). It may also be worth designing a randomized clinical trial to identify the effects of caregiver support when a person with ADRD in enrolled in a clinical trial.

\section{Recommendation \#4: Include people with ADRD and caregivers in the research}

design process: In the Gilmore-Bykovskyi et al. (2019) systematic review on improving dementia research recruitment among disadvantaged populations, it was noted that none of the papers that described strategies to improve research participation included bringing community members into the design process, and recommended researchers begin doing so. In this particular study, interviewees had mixed feelings on incorporating community members with and without ADRD. Some interviewees noted that they do not include community members because they pose challenges, particularly in the context of a person with ADRD participating in the research process:

Researcher: "Those are challenging because I think they sound good, but if you think about it, how can one person from a community represent the community? I mean, you take it as input, but I think that it's hard to get the voice of a community from a single community representative.

Healthcare provider: "For the things I've done, we haven't included individuals with dementia ... with dementia, people often lack insight, they often have enough memory impairment that they won't remember a previous conversation. So, you can't build on what you've done previously and it's just a much more limited interaction."

Others viewed community members as experts, highlighting that caregivers and people with ADRD are the only ones with the true expertise in living with or caring with someone with ADRD. 
Researcher: "If we have a meeting, I make sure that we don't have 7 [researchers] and 3 community members on the other side of the table ... We are conscious about power imbalance ... We always want to approach it as 'we want to learn from you' ... we want to say, 'look, you're the experts, help us learn from you."”

Advocate: "We have people living with cognitive impairment and change ... They are [on the board] because they're an expert. They know what it means to live with this disease every single day. They know what it means to care for someone's disease or to watch their husband change before their eyes. They know what it means. They're an expert and that's who policymakers want to hear from."

One pathway to include people with ADRD and caregivers in the research design process may be to start with websites that are designed for finding available research

opportunities. Several interviewees noted that the study websites that they used to search for trials or join a registry were difficult to navigate:

Caregiver: "One of the people at the [organization] said their website is a nightmare to navigate, so I doubt if I would have looked and found [the clinical trial] there."

Caregiver: "[The study search websites] are probably for medical professionals who are used to doing it all the time. I talked to the family doctor. I say, 'How come you can find this stuff and I can't?' She says, 'Because I do it every day, and I know exactly how these people think when they set these websites up ... You don't do it every day. You're not familiar with how it works. '"

At least one caregiver had ideas to improve online study search functionality and ability:

Caregiver: "[The organizations] send you an email and say .... 'Go to this website, fill this thing out.' ... [Then] they look at the questionnaire and they say, 'Okay, now if this answer was yes, then they might be a candidate,' or that type of thing. They could at least establish an initial contact ... Because right now, searching through a whole bunch of websites looking for research, studies where the criteria might meet what she has ... doing that is very time-consuming."

This example illustrates how people with ADRD and caregivers provide needed expertise based on their lived experiences in the research design process. They identified barriers 
affecting their ability to search for research studies and suggested a strategy to mitigate that barrier.

When asked about being on an advisory board where caregivers and people with ADRD collaborate with researchers and staff, most people with ADRD and caregivers described these boards as not important in their decision to participate in research. Some caregivers mentioned wanting to join an advisory board in the future but were unaware of current opportunities. A small number of people with ADRD said they would be interested in serving on an advisory board. One interviewee was a former neurologist diagnosed with Alzheimer's, who speaks to medical students about treating individuals with dementia. This person is also part of a research advisory board for the Alzheimer's Association, but was unable to join their advocacy board due to policies regarding caregivers:

Person with ADRD: “The Alzheimer's Association invited me to join an advocacy group; I said I'd be happy to do it but they required a partner because they didn't want people to travel on their own ... I don't know how much longer I'll be able to do that, but right now that's not an issue. But that was something that was etched in stone ... [so] they said how about a different division?"

Notably, several people with ADRD felt that they would not be able to contribute:

Person with ADRD: “My brain isn't doing very well. I don't think that's something that would help anybody with me getting confused. I don't need to help people when I can't [help] myself."

Organizations may benefit from recognizing the expertise that people with ADRD bring to the table. People with ADRD can be empowered through an organization's inclusive decision-making processes, such as inviting them to join advisory boards if they are competent to participate fully to the extent to which they are capable. Some interviewees 
with ADRD felt their contributions would be unhelpful, so an invitation with an explanation of why they are being invited might serve as a way to empower them personally. In doing so, this may lead to a change in the social construction of people with ADRD by empowering them within their community as well as increasing equity by having their voices and expertise included in the research design process.

As noted in Chapter 3 and the methods section of this chapter, a CAB was established for this study. In addition to professional members, the CAB included people with ADRD, caregivers, and older African Americans. In addition to helping develop study materials and interpreting the results, they provided numerous insights, such as using the terms "Alzheimer's disease" and "dementia" instead of "memory loss," describing how compensation is helpful but researchers need to consider how it may affect their benefits, and articulating what learning styles were most helpful to them (e.g. visual versus auditory), which led to multiple ways that knowledge was disseminated before, during, and after the $\mathrm{CAB}$ meeting. Additionally, at every $\mathrm{CAB}$ meeting debrief, the members identified what was positive about the session and what could be adjusted to better suit their needs.

Further, when provided with a list of factors affecting ADRD clinical research participation, which was developed based on providers, researchers, and advocates input, every Phase 2 interviewee provided insights on what factors were important, which were not, and how the list could be improved. They demonstrated that individuals with ADRD and caregivers have the expertise to improve study design even in cases where they believed they did not have the capacity to do so. 
Conclusion: The conceptualization of Action Area 3 -- creating healthier, more equitable communities -- is generally applied through the lens of the built environment, social and economic conditions, and policies (Dubowitz et al., 2016). In the context of this study, the lens of equity is achieved through the inclusion of people with ADRD and caregivers in creating policies and processes for ADRD research. Findings from interviews recognize the necessity of caregiver participation in ADRD research but also highlight the sometimes negative position they may be placed in. Recognizing caregivers as full participants rather than study partners offers the ability for researchers to provide the caregivers with some of the benefits they need and could motivate caregivers to be involved in research. Including caregivers and people with ADRD in the research design process provides opportunities to remove barriers and strengthen resources that address the needs of their community while encouraging participation. It also empowers people with ADRD and their caregivers by recognizing and respecting their expertise, further reducing inequities.

\section{Culture of Health Action Framework Action Area 4: Strengthening and integration of health services and systems}

The clinical research, advocacy, and health sectors have similar goals of improving the experiences of people with ADRD and their caregivers as well as discovering an ADRD prevention and treatment (NIH 2018; Alzheimer's Association, 2019). This action area is conceptualized as including access to care, balance and integration, and consumer experience (Martin et al., 2016). In the context of ADRD research, this can be applied in the realm of access to research opportunities, balance and 
integration of research services, and participant experience. Individuals report being more open to clinical research participation if organizations are more accommodating by offering flexible scheduling, fewer in-person visits with more opportunities for video conferencing, and free or subsidized modes of transportation (Danner et al., 2011; Law et al., 2014; Friedman et al., 2015; Mahon et al., 2016). Study opportunity knowledge could also be fostered by modifying both large-scale and local online registries and clinical research websites, as noted in Recommendation 4. These sites and registries, including TrialMatch, ClinicalTrials.gov, and local research registries, offer information about or the option to be notified about study opportunities but are inaccessible to those who are scientific or Internet illiterate or with limited time to navigate the cumbersome sites. The relationship between organizational staff and a person with ADRD and their caregiver often contributes to the decision to participate in research.

\section{Recommendation \#5: Offer alternative options to reduce participation burden:}

Interviewees noted multiple factors affecting their ability and motivation to participate in ADRD clinical research. Time spent driving, using the internet to search for study opportunities, and participating in study activities were frequently discussed:

Researcher: "So the sponsor sends somebody to do [quality assurance testing] on one specific machine, so we can't even just send them to one facility, it has to literally be one specific machine that was [tested] by them, so that they're scanned under the same protocol each time on the same machine, really just anything to reduce variability. That would help if we could send people to other places."

Caregiver: "We drive out [across town] for an optometry appointment, and have to drive back, and it's just like that was what really put her off to it. And me, too ... I mean, they had one place for CT and one place for MRI ... [and then a third place for] optometry ... [Then], she had a hearing test at another place." 
Caregiver: "There are a lot of opportunities for us to do things that enrich what are still good years. If it was going to be some intervention that would interfere with that or gobble up a lot of our time, that would be a substantial negative."

Research organizations' strategies may benefit from modifications to reflect community needs. Based on the interviews, this could include presentations at ADRD and caregiver support groups, making materials reflective and culturally responsive to the community that researchers wish to work with, and providing additional incentives for caregivers. This also applies to industry sponsors that may be receiving suggestions from coordinators or community members about the best recruitment strategies but continue to adhere to their original processes. Willingness to accommodate their schedules and needs, such as requiring fewer in-person visits, reducing or removing the time spent or distance traveled, or providing resources or supports specific to the caregiver, may help in increasing their ability and motivation to participate in ADRD research. For example, including more secondary sites for specialty testing (e.g. optometry or CT scans), or working with home health agencies to reduce the number of clinic visits, could address travel-related burdens and may increase participation.

\section{Recommendation \#6: Evaluate and improve relationships between providers/}

researchers and patients/participants: In Phase 1, several interviewees described the fear that some providers have when encountering a person with signs of dementia:

Healthcare provider: "I think on the provider's side ... it's just uncertainty and feeling like there's nothing for them to do. So why make the diagnosis? Because they could be wrong. When in fact, there are things to do. Get your affairs in order, advanced directives, you know? There are lots of things to do. Do a bucket list..." 
This sentiment was echoed by several of the Phase 2 interviewees, who noted negative interactions with their provider. Less obvious dementia symptoms, such as trouble concentrating at work, were noticed more often by the person with ADRD who felt their symptoms were minimized or dismissed by providers, despite continued concerns, resulting in a delay in the diagnosis. Several interviewees noted interactions that led to uncertain diagnoses or diagnoses received in an unprofessional manner:

Person with ADRD: "I knew that there were some issues. And so I went to my family care physician and I said, 'You know, I just can't remember things like I used to.' And he just discounted it ... So then I went and spent another few years after that and it just kept continuing to get worse."

Person with ADRD: "At first [the neurologist] was sort of dismissive, "Oh, I'm sure you're just being neurotic," kind of message ... [After testing], he comes back, he throws down the papers on the table and he said, "You're right! You do have a problem!" ... I just kind of sat there in stunned silence. I just thought, "Wow. Do you let all of your patients know the results that way?"

Some noted that relationships with their research staff were different from their

experiences with their providers, and had positive things to say about the staff:

Caregiver: "The staff, research assistants, are just fantastic. They really do everything they can to make it easy for you."

Person with ADRD: "I can't think of anything that [research staff] can do better ... It's just superb. It's better than even dreamed of. And they make you feel worthwhile ... They look at me, they listen, they reflect back, they communicate beautifully."

Others noted that the relationship between their provider or research staff affected their decision to keep seeing them:

Caregiver: "If [research staff] treated him or us more as a number instead of a person, that would definitely be a factor [in participating]. But we haven't seen that ... these days, you go in for [a doctor's] appointment and you feel like a number instead of a person." 
Conversely, others were apprehensive because of previous bad experiences:

Person with ADRD: "If you don't care anymore about your patient than [having the secretary call to give the diagnosis], he needs to be doing something else ... He was so lackadaisical, like, 'Geez, I've done this test for so many times'... I don't know if I should [go see another neurologist]. I'm scared to go to one now."

These quotes exemplify how important the experience and interaction between staff and patient/participant is in the decision to participate in research or seek treatment. These interviewees had positive experiences with research staff, but that cannot be generalized for all ADRD research participants.

Conclusion: In the context of ADRD research, this study conceptualizes Action Area 4 through access to research, balance and integration of research services, and participant experience. Developing alternative options to reduce participant burden provides greater access and integration of research services, and improves the experience and quality of participating in ADRD research. Similarly, the relationship between a provider or researchers and their patient or participant substantially influences their experience.

\section{Findings that do not align with the Culture of Health Action Framework}

Two findings did not align with the Culture of Health Action Framework. The first involves the number of people who will not participate in research due to their concerns about taking study drugs. Ten of the 24 Phase 2 interviewees noted that they would not participate in a study that required them to take study drugs. In some cases, learning from local ADRD research champions, particularly those who have participated in drug trials, may inform people with ADRD and caregivers of the purpose, benefit, and concerns of participation. Five of those Phase 2 interviewees described a history of taking 
a new medication that negatively impacted their health and did not want to repeat that experience, especially if the drug was not proven to treat their dementia. In these cases, the Culture of Health Action Framework would not be helpful for increasing their likelihood of participating in an ADRD drug intervention. However, the framework could still be used to improve their motivation and ability to participate in alternative types of ADRD research opportunities that do not require taking an investigational drug.

The second finding concerns the number of people who are ineligible or forced to withdraw from a study. Three of the 12 people with ADRD interviewed noted that they had been ineligible for, or withdrawn from, studies for three reasons: age, neuropsychological testing scores, and/or for scoring too high on screening tests after receiving an intervention. Additionally, one caregiver noted that she was ineligible to participate in an ADRD prevention study for herself because she did not have a study partner, despite having normal cognitive function. Of these four reasons, age is the only ineligibility factor that has been addressed due to a policy that promotes equity in ADRD research by removing arbitrary age exclusion criteria $(\mathrm{NIH}, 2017 \mathrm{a})$. The other three exclusionary reasons are necessary to ensure study validity, intervention efficacy, and the overall safety and wellbeing of the participants. Because of their necessity, they are not likely to be adjusted or modified.

Despite these two findings not having a good fit with the Culture of Health Action Framework, the majority of the findings do fit within the framework. Thus, this framework remains a useful tool for conceptualizing factors and organizing recommendations to improve ADRD clinical research participation. 


\section{Synthesis and Conclusion}

The goal of finding a treatment or prevention for ADRD by 2025 is contingent on having enough people with ADRD and study partners who are able and willing to participate in clinical research. For Oregonians with ADRD and their caregivers, there are many policy, system, organizational, and personal barriers potentially standing in the way of capability and motivation to participate in ADRD clinical research. However, the recommendations presented here may mitigate these barriers. The Culture of Health Action Framework was used to frame this research; these six recommendations were derived from the expertise of the interviewees and were then applied to this framework. They can serve as a map to build capacity and partnerships, recognize and mitigate barriers caused by structural racism, help research organizations conduct more outreach to motivate community members and increase ADRD clinical research enrollment.

There are several limitations to this project. First, the sample size is small $(n=33)$ and not fully representative of one metropolitan area. However, saturation was reached through these interviews. These findings are specific to the Portland, OR metropolitan area. They may be contextually relevant for similar communities, but are not necessarily generalizable nor are they intended to be, in the quantitative sense. Rather, these findings can be transferable to other researchers, clinicians, or advocates in other metropolitans areas if they believe these findings may be applicable to their setting and specific context (Smith, 2017). 
Second, there is sampling bias in this study. Though Phase 1 interviews were designed to be organizationally representative, all but one of the healthcare providers were geriatricians and there were no providers who specifically focused on primary care. This is because the investigator relied upon referrals from key informants and community advisory boards, and nearly all provider referrals were for geriatricians. Further, one of the three local ADRD research organizations did not respond to requests for interviews. The study also did not include interviews with local county government employees despite the fact that county governments in the Portland metropolitan area provide services for people with ADRD and caregivers. This is because the investigator was not referred to county employees for Phase 1 interviews by either the $\mathrm{CAB}$ members or other key informants. These county agencies were discussed by advocates, but not by providers and researchers, so it is possible that the services provided by the counties are unknown to local health and research organizations. In Phase 2, all but one interviewee was white, despite efforts to recruit African Americans. Factors that specifically affect African Americans were not captured in this study. It is likely that a more robust discussion of built environments and shared values, specifically around local ADRD research studies that target African Americans, would have emerged from the findings if there had not been this bias. This sampling bias is discussed in detail in Chapter 7 .

Confirmation and response biases also exist in this study. Interviewees may have censored themselves due to the audio-recording of interviews and may have focused on what they perceived the interviewer wanted to hear, resulting in response bias. Confirmation bias is also likely given that the investigator was the single coder and drew 
upon the conceptual framework and the literature. The investigator mitigated some of this bias by writing field notes immediately after each interview, a conscious effort to selfassess when analyzing codes and themes, and $\mathrm{CAB}$ member-checking and interpretation of analyses. Furthermore, while there are no conscious biases due to the researcher's identity, it is important to acknowledge that subconscious biases are possible due to her identity as a female academic who is a proponent of ADRD research and community inclusion and may have affected the rapport with interviewees. The consequences of these biases are discussed further in Chapter 7.

This study contributes to the literature by providing recommendations to improve dementia clinical research recruitment and by providing a deep and rich understanding of the experiences of people with ADRD, caregivers, clinicians, researchers, and advocates. Additionally, these recommendations derive from the voices of interviewees and are organized using the Culture of Health Action Framework, applying the framework in a unique manner. Further, the study successfully demonstrated that including people with dementia and caregivers on an advisory board can be valuable, and improved the study design and interpretation of findings. Through this study, the potential exists to increase research participation at the local level to the point that it increases the national rate of recruitment, and ultimately helps to achieve the US Congress's goal of finding a prevention or treatment for ADRD by 2025. This study should be repeated in a larger context or as pilots in other communities to determine contextual relevance and generalizability for other areas. Further, strategies implemented as a result of the recommendations provided here should be routinely evaluated to determine their 
feasibility and effectiveness. Future research related to ADRD should consider the larger context - these recommendations are not only about finding a way to increase participation in ADRD clinical research studies, but more importantly about the larger goal of finding a prevention, treatment, or cure for ADRD. 


\section{CHAPTER 7: CONCLUSION}

This final chapter offers a detailed description of the conclusions of this dissertation. It is organized as follows:

a) Restatement of the study's purpose and a summary of the findings;

b) Assumptions and limitations;

c) Plans for dissemination of the findings;

d) Implications for future research; and

e) Conclusion.

\section{Overview of the Study Purpose and Summary of Findings}

The purpose of this study was to identify organizational, system, and policy factors affecting ADRD clinical research participation in the Portland, Oregon metropolitan area. This research question was addressed through three aims.

\section{Aim 1: Identify organizational, system, and policy factors that impede or enhance clinical research enrollment among people with ADRD.}

Aim 1 was addressed through nine semi-structured interviews with local clinicians, researchers, and advocates who were identified through referrals from key informants and a Community Advisory Board. Organizational, system, and policy factors affected ADRD clinical research participation in the local community. Federal policy attempts to streamline ADRD research studies have not resulted in the intended outcomes at the local level. People with ADRD and caregivers lacked awareness of research 
opportunities. In some cases, this was due to a lack of provider knowledge of ongoing clinical research. In others, it was due to organizational directives preventing providers from discussing research opportunities. At times, providers preferred not to refer patients to research opportunities. Research opportunities were not shared in places where people with ADRD and their caregivers are likely to congregate, such as support groups. Interviewees, many of whom participated in professional partnerships, were often hesitant to join community collaborations or formal partnerships. This reduced the likelihood of building a relationship and shared resources with people with ADRD and caregivers.

Many interviewees, mostly clinicians and some researchers, were wary of including community members, with or without ADRD, on their advisory boards. Reasons ranged from not receiving true representation from the community to people with ADRD negatively affecting the progress of the advisory board. Advocates and other researchers spoke positively of including people with ADRD and caregivers, noting that they were the only true experts because they had the lived experiences.

\section{Aim 2: Describe personal factors that persuade or dissuade individuals with ADRD from enrolling in clinical research.}

Personal factors affecting ADRD clinical research participation in the Portland, Oregon metropolitan area were identified through interviews with twelve people with $\mathrm{MCI}$ or ADRD and their caregivers $(\mathrm{n}=24)$. Interviews explored factors affecting the interviewees' ability and motivation to participate in research. Half of those interviewed had participated in dementia clinical research; participants with ADRD must have had a 
clinician diagnosis. Several interviewees noted their provider initially dismissed their symptoms of ADRD, with two citing unprofessional behavior. There was a lack of knowledge about research opportunities in the local Portland area. Many interviewees were unaware of dementia studies, which was surprising as many were recruited from organizations with rich knowledge of research opportunities.

One's perceived value of ADRD research did not increase their likelihood of joining a study. Many caregivers either did not have or did not want to use their limited free time to be a study partner; however, several caregivers noted that they would take the time to participate if the study provided some sort of caregiver benefit to ease their burden. Benefits might include listening to the caregiver's challenges, providing or referring to individual therapy or support groups, caregiver education, or respite services. Ten of 24 Phase 2 interviewees noted that they would not participate in a study that required them to take an investigational drug, but all expressed willingness to participate in other types of ADRD studies.

\section{Aim 3: Develop motivational strategies and policy recommendations based on the findings from Aims 1 and 2.}

The discussion of Aim 3 presented recommendations for local clinicians, researchers, and advocates to facilitate ADRD clinical research participation. Six strategies emerged from the qualitative interviews, $\mathrm{CAB}$ discussions, and an extensive review of the literature: 1) identify and promote local champions for ADRD clinical research participation; 2) promote policies and processes that incentivize cross-sector collaboration; 3) recognize caregivers as full research participants; 4) include people with 
ADRD and caregivers in the research design process; 5) offer alternative options to reduce participation burden; 6) evaluate and improve relationships between healthcare/ research staff and patients/participants.

The Culture of Health was adapted and applied as a guiding framework to help conceptualize factors affecting ADRD clinical research participation and to organize the strategies within the framework's four action areas. Using the Culture of Health Action Framework, the six recommendations can serve as a map to build capacity and partnerships, recognize and mitigate barriers caused by structural racism, help research organizations conduct more outreach to motivate community members and increase ADRD clinical research enrollment.

\section{Assumptions and Limitations}

As with any research, there are some assumptions and limitations that influence the interpretation of the findings and conclusions of this dissertation study.

\section{Assumptions}

Three assumptions were made. First, the investigator correctly assumed that clinicians, researchers, advocates, and dyads of people with ADRD and their caregivers were willing to participate in semi-structured interviews. Second, there was an assumption that the investigator would be able to successfully recruit African Americans for Phase 2 of the study given her connections with organizations that work with older African Americans in the Portland area. The investigator was not successful; this is discussed in the following subsection. Third, there was an assumption that individuals 
have the capacity and motivation to join a CAB. This assumption was accurate. Lastly, there was an assumption that the CAB would confirm the investigator's findings. This also was accurate.

\section{Limitations}

There are several limitations to this dissertation research, including a lack of African American representation, and issues of generalizability, sampling bias, response bias, and confirmation bias.

Lack of African American representation: This study's recruitment goal of 50\% African Americans for the Phase 2 interviews was unmet; no African Americans were interviewed for Phase 2. Recruitment was attempted through CAB referrals to four African American community organizations, two OHSU African American cohort studies, a PreSERVE member with several friends who care for a friend or family member with ADRD, and by sharing flyers and attending three community events that catered to older African Americans. Of the four African American community organizations suggested by the $\mathrm{CAB}$, three of the contacts did not respond and one ceased communication. Of the respondents from the two OHSU studies, none of the individuals had a clinical diagnosis of MCI/ADRD. Of the five participants that responded to an email from the study investigator, none were caregivers. The investigator brought flyers and interacted with attendees at community events. Two attendees reported being interested but did not respond to follow-up. Limitations due to lack of African American Phase 2 interviewees as well as steps to recruit and interview African Americans for future studies are described below. 
Additional limitations: In addition to the lack of African American representation, other limitations must be acknowledged in interpreting study findings. This includes generalizability, sampling bias, response bias, and confirmation bias.

Generalizability: This was a small pilot study in one midsized city based upon 33 interviews, but saturation was reached in both phases of interviews. These findings are specific to the Portland, OR metropolitan area but may be contextually relevant for similar communities. They are not necessarily generalizable nor are they intended to be in the quantitative sense. Rather, these findings can be transferable to other researchers, clinicians, or advocates in other metropolitan areas if they believe these findings may be applicable to their setting and specific context (Smith, 2017).

Sampling bias: Although saturation was reached, 33 interviewees are not representative of all of the ADRD clinicians, researchers, advocates, people with ADRD or caregivers in the Portland, OR area, resulting in possible sampling bias (Kovera, 2010). Phase 1 sought to include representation of numerous organizations by including interviewees from organizations of varying sizes and structures. Nine representatives from six organizations of varying sizes and structures were included, but the study did not include representatives from two local healthcare systems, independent health clinics, and one of the three ADRD research organizations. Had these representatives been included, it is possible that there would have been more discussion of different funding sources, community collaborations, and interactions with people with ADRD and caregivers. Three of the clinicians identified as geriatricians and one identified as a neurologist. Not having primary care providers omitted unique findings of being the first healthcare 
provider to see a person complaining of dementia symptoms, as compared to a neurologist or geriatrician who has received a referral.

Additionally, this study did not include representatives from local county government offices on health and aging, who provide social services and supports for people with dementia and caregivers in the Portland metropolitan area. This was because they were not recommended by professional contacts nor by the CAB as a Phase 1 interviewee but rather suggested as a recruiting source for Phase 2 interviews. These additional perspectives may have provided insights into the value of social gatherings and fostering a community among people with ADRD and caregivers. Representatives from pharmaceutical or other industry groups were not included because they do not directly interact with people with ADRD or caregivers. Given the leverage they have over research study design, their insights could provide a better understanding of their organizational roles and structures, funding, and decision-making processes.

Sampling bias is also likely in Phase 2 interviews. Phase 2 interviews included just 24 people with dementia and caregivers in a state with over 67,000 people diagnosed with Alzheimer's disease and an estimated 200,000 caregivers (Alzheimer's Association, 2019a). Interviewees who had not participated in ADRD clinical research were involved in some type of group activity related to ADRD or caregiving, resulting in a lack of inclusion of people who are less connected to ADRD supports and services. This may have omitted insights on factors affecting their decisions regarding engaging in research or group supports. Working with a 100\% non-African American sample versus 50\% nonAfrican American sample meant that some factors described in the literature, by Phase 1 
interviewees, and by the $\mathrm{CAB}$ were not identified. African American interviewees might have discussed how egregious acts conducted by researchers, Oregon's history of mistreating African Americans, current gentrification, and lack of formal health service access and utilization negatively impacts their decision to participate in ADRD clinical research (Hannah-Jones, 2011; Parks, 2012; Multnomah County Health Department, 2014; Geiling, 2015; Bonds \& Lyons, 2017). It also left out a robust discussion from African Americans who do participate in ADRD research about why they choose to join a study. These findings would have been useful for identifying culturally competent and community engagement practices to increase ADRD research participation.

Response bias: The researcher identifies as an academic, is employed at a large research organization and is a proponent of research, all of which could have affected power dynamics between the researcher and interviewees. It could also have led to response bias, where interviewees may answer questions in a way that seems more agreeable (Kovera, 2010). For example, individuals who have negative perspectives of academics and research may have felt they needed to speak positively about research in order to please the investigator. Additionally, it is possible that stronger connections could have been made with interviewees whose views align with the researcher's, which could have led to more sharing of details about interviewees' perspectives, as compared to those who are not proponents of dementia research. Possible response bias may have led to a lack of robust discussion on more negative perspectives of research or relationships with research staff. 
Confirmation bias: Per Portland State University and Health System \& Policy Ph.D. program requirements, only one researcher coded and analyzed the data. The investigator used the Culture of Health Action Framework as a conceptual framework and drew on the literature and various theories for coding and analysis. This increases the risk of confirmation bias, or the predisposition to value evidence that confirms preconceived notions and dismiss evidence to the contrary (Karson \& Goodwin, 2010). It is possible that the investigator ignored data that did not fit with the framework or literature, and missed important findings. To mitigate these risks, the researcher took field notes after each interview to capture various emerging themes and findings, particularly those that were surprising and not found in the literature. Additionally, the researcher worked with the $\mathrm{CAB}$ to member-check and interpret the findings by identifying and describing what was interesting, surprising and/or missing.

Despite these limitations, the qualitative design elucidated contextual factors affecting ADRD clinical research participation in Portland, OR that have not been described elsewhere, and could not have been gleaned from quantitative analyses alone.

\section{Dissemination of the Research}

Originally, the researcher planned to host a community event so people with dementia, caregivers, and other interested community members could learn about findings and recommendations from this study. The $\mathrm{CAB}$ noted that caregivers and people with ADRD are unlikely to attend an event that was specifically held to describe the findings, even if it did offer other resources. They recommended that the findings be shared in a 
multitude of ways to serve varying populations. The first is to join presentations that discuss healthy aging and dementia, such as those hosted by health clinics, advocacy groups, community organizations, or the local government. The second strategy is to speak at professional conferences that are designed for local caregivers, such as the McGinty Caregiver Conference. The third is to speak at professional conferences that are designed for local dementia professionals, such as the Oregon Gerontological Association. The fourth is to staff information booths at community events specific and non-specific to dementia, ranging from neighborhood festivals to the annual Alzheimer's Walk. These recommendations are in addition to making presentations at academic conferences and submitting manuscripts to relevant journals.

\section{Implications for Future Research}

Findings from this dissertation suggest several topics for further research. First, this study could be expanded to include more interviewees in the Portland metropolitan area. Recruitment could be conducted through research organizations, advocacy groups, clinics, county offices, support groups, and memory cafes. Single or dyadic interviews may be resource-prohibitive due to the time and money required to conduct and analyze interviews with dozens of individuals. In these cases, focus groups or surveys could be used to build upon the findings identified in this dissertation. Strategies developed through this study, such as establishing local champions to promote ADRD clinical research participation, should be implemented, routinely evaluated and modified to fit community needs and increase ADRD clinical research participation 
Second, pilot studies could be conducted in other communities to identify how contextual factors affect dementia research participation. Identifying how organizational, system and policy factors affect the ability and motivation of a person with dementia to participate in local dementia clinical research opportunities cannot be generalized to a larger study in a different geographic area. Larger studies can be developed after pilot studies, and as noted previously, strategies developed through this study should be implemented, routinely evaluated and modified to fit community needs and increase research participation.

Third, this study should be expanded to involve communities of color. This requires extensive preparation through coalition building with community leaders and the development of a trusting relationship with the community. As previously mentioned, this study attempted to focus on African Americans because of their increased likelihood of developing ADRD but the investigator did not successfully recruit interviewees. Based on suggestions from the $\mathrm{CAB}$ and the literature, three recruitment strategies should be used in a future study to include African Americans in this research. First, efforts should be made to reach out and work with African American churches in the local area. Many older African Americans attend church services, and many churches have health ministries that can work to promote health and reduce disparities among their parishioners (Levin, 1984; Holt et al., 2018). Another strategy would be to work with local community health clinics, such as North by Northeast, a community health clinic in Portland that focuses on African American health, to recruit for future studies. A third strategy would be to contact local African American clinicians who are identified on 
websites that promote African American medical professionals in Portland. A fourth strategy would be to contact and work with Portland's Black-owned businesses to hang flyers, meeting customers, and encourage word-of-mouth referrals.

Other communities of color, including older Latinx, Asian Americans, and Native Americans, are also more likely to develop ADRD as compared to non-Hispanic white adults. Therefore, the specific inclusion of communities of color should be part of future studies. In addition to coalition building and development of a trusting relationship, this will require funding resources for bilingual, culturally competent staff and translation of materials. Strategies for recruitment from communities of color, described above, should be modified and applied for these other communities.

Fourth, studies should be conducted to determine if involving caregivers as full participants and providing them with caregiver supports increases recruitment and retention. An advisory board of current and former caregivers can identify the best options for what they need from the research team. Researchers may be hesitant to offer caregiver supports because it is difficult to ascertain if improvements are due to the study intervention for the person with dementia or because of the caregiver support. It could be beneficial to develop a randomized clinical trial where some study partners receive caregiver supports and others do not, and thus test if these supportive interventions improve retention.

Fifth, studies can be conducted to evaluate the success of various research websites and registries in recruiting new participants into studies, as well as how they can be better designed. Study opportunities can be found through specific research 
organization websites (e.g. a research university), through federal websites (e.g. Clinical Trials.gov), and local and global dementia-specific websites and registries (e.g. ACTNOW, Brain Health Registry, TrialMatch, Alzheimer's Prevention Initiative). Evaluations can include how to define "success" (e.g. website search clicks or registry signups, initial contacts, screening visits, enrollments), which type of website design is most successful, and how to improve design and functionality of websites. Advisory boards of people with ADRD, caregivers, and community members, focus groups, and/or surveys can be utilized to identify website strengths and weaknesses and develop solutions.

There are theoretical implications of this research study. The Culture of Health Action Framework was applied in a novel way for ADRD clinical research. This framework served as a map to build capacity and partnerships, recognize and mitigate barriers caused by structural racism, help research organizations conduct more outreach to motivate community members and increase ADRD clinical research enrollment. Elements of transaction cost theory, civic engagement, and contingency theory can be used by research organizations to increase the ability and motivation of people with ADRD and caregivers to participate in research. Transaction cost theory describes the cost of participating in a study. Civic engagement describes how ability and motivation affect the decision to participate in a study. Contingency theory describes how, and to what extent, organizations can address the needs of people with ADRD and caregivers.

There are a number of research implications that can be directed to specific groups in order to facilitate ADRD research recruitment and retention. Researchers can 
partner with clinics and community organizations to briefly present research opportunities and share flyers, particularly those that do not require study drugs. Researchers can also develop studies that evaluate their recruitment strategies and identify which are effective and which can be improved. They can also create advisory boards with people with ADRD and caregivers to improve recruitment and study design processes. Federal agencies could earmark some of the 2.8 billion dollars designated for dementia research to fund studies that focus on recruitment and retention. In recent years, the NIH and the Patient-Centered Outcomes Research Institute (PCORI) have created formative efforts to increase community outreach and collaboration to improve clinical research design, recruitment, and outcomes (PCORI, 2013; NIH, 2018). PCORI recently funded a two-year award to LiveWell, a dementia service provider in Connecticut (PCORI, 2019), to increase the capacity of people living with dementia and their caregivers and reduce barriers to their engagement in patient-centered research (LiveWell, 2018). There are many advocacy groups that advocate for this type of collaboration, such as the Alzheimer's Association, Us Against Alzheimer's, and the Dementias and Neurodegenerative Diseases Research Network (DeNDRoN) in England. ADRD research organizations have not encouraged this type of collaboration to the extent that advocacy groups have done, possibly due to reasons articulated in Phase 1 interviews and described in Chapters 4 and 6.

Advocates, healthcare providers, and policymakers can also facilitate ADRD clinical research recruitment. Advocates can share research opportunities with constituents and list local research opportunities on their websites and resource packets. 
Healthcare providers should view research studies as another resource and include materials in referral or resource packets for patients, or share flyers and brochures from outside of their clinics in their waiting rooms. In addition to earmarking designated funds for recruitment studies, policy-makers can remove policies that forbid discussions of research and promote marketing campaigns that publicize dementia diagnoses and research participation.

\section{Conclusion}

The US Congress's deadline to find a prevention or treatment for ADRD by 2025 is only a few years away. In addition to the billions of dollars that Congress has earmarked for dementia research, the NIA released a report highlighting strategies to increase ADRD clinical research participation (NIA, 2018). This study addressed their fourth strategy, developing a science of recruitment, through a first step of identifying factors affecting clinical research participation within the local Portland, OR metropolitan area. Subsequent steps for researchers and policy analysts include developing and implementing policies to improve research recruitment and retention, evaluating and modifying policies as needed to fit the needs of the community, and achieving the overarching goal of increasing research participation.

This study contributed to the literature by providing contextually relevant factors affecting clinical research participation as well as providing recommendations to improve recruitment. Further, this study successfully demonstrated that including people with dementia and caregivers on an advisory board can be effective, and the use of a CAB 
improves the study design and interpretation of findings. Through the findings and conclusions of this study, the potential exists to increase research participation at the local level to the point that it increases the national rate of recruitment, and ultimately helps to achieve the US Congress's goal of finding a prevention or treatment for ADRD by 2025. 


\section{REFERENCES}

$21^{\text {st }}$ Century Cures Act, Pub. L. No. 114-255, 130 Stat. 1033 (2016).

Abrahamson, K., Clark, D., Perkins, A., \& Arling, G. (2012). Does cognitive impairment influence quality of life among nursing home residents? The Gerontologist, 57(5), 632-640.

Adams, M., Caffrey, L., \& McKevitt, C. (2015). Barriers and opportunities for enhancing patient recruitment and retention in clinical research: findings from an interview study in an NHS academic health science centre. Health Research Policy and Systems, 13(8), 1-9.

Adelman, R.D., Tmanova, L.L., Delgado, D. Dion, S., \& Lachs, M.S. (2014). Caregiver burden: a clinical review. Journal of the American Medical Association, 311(10), 1052-1059.

Aging and Disability Resource Center. (2017). Adult Day Services. Retrieved from: https://adrcoforegon.org/consite/explore-adult-day-services.php.

Ahmed, R.M., Paterson, R.W., Warren, J.D., Zetterberg, H., O'Brien, J.T., Fox, N.C., Halliday, G.M., \& Schott, J.M. (2014). Biomarkers in dementia: clinical utility and new directions. Journal of Neurology, Neurosurgery, and Psychology, 85(12), 1426-1434.

Alzheimer's Association. (2019a). Alzheimer's Association Report: 2019 Alzheimer's disease facts and figures. Alzheimer's \& Dementia, 15(3), 321-387.

Alzheimer's Association (2019b, December 16). Congressional leaders agree on $\$ 350$ million Alzheimer's and dementia research funding increase. [press release]. Retrieved from: https://www.alz.org/news/2019/congressional-leaders-agree-on\$350-million-alzhei.

Aminzadeh, F., Molnar, F.J., Dalziel, W.B., \& Ayotte, D. (2012). A review of barriers and enablers to diagnosis and management of persons with dementia in primary care. Canadian Geriatrics Journal, 15(3), 85-94.

Anderson, A., Borfitz, D., \& Getz, K. (2018). Global public attitudes about clinical research and patient experiences with clinical trials. JAMA Network Open, l(6), e182969.

Anstey, K.J., von Sanden, C., Salim, A., \& O'Kearney R. (2007). Smoking as a risk factor for dementia and cognitive decline: A meta-analysis of prospective studies. American Journal of Epidemiology, 166(4), 367-78. 
Aranda, J. (2017). Multisystemic approach to dementia care research: Person; family/ close/informal care; care supports/services; large institutions/regulatory policies. [powerpoint presentation] 2017 NIH Research Summit on Dementia Care. Washington, D.C.

Arras, J. (2008). The Jewish Chronic Disease Hospital case. In: Emanuel, E.K., et al. (Eds.) The Oxford textbook of clinical research ethics (pp. 73-79). New York, NY: Oxford University Press.

Association for Frontotemporal Degeneration. (2018). Disease Overview. Retrieved from: https://www.theaftd.org/what-is-ftd/disease-overview/.

Axovant (2017, September 26). Axovant announces negative topline results of intepirdine phase 3 MINDSET trial in Alzheimer's disease. Retrieved from: http://investors.axovant.com/node/7286/pdf.

Balazs, C.L. \& Morello-Frosch, R. (2013). The three Rs: How community-based participatory research strengthens the rigor, relevance, and reach of science. Environmental Justice, 6(1), 9-17.

Balintfy, J. (2017). New NIH consortium award to enhance clinical trials for Alzheimer's disease, related dementias. Retrieved from: https://www.nia.nih.gov/news/newnih-consortium-award-enhance-clinical-trials-alzheimers-disease-relateddementias.

Ball, M.M., Perkins, M.M., Hollingsworth, C., Whittington, F.J., \& King, S.V. (2008). Pathways to assisted living: The influence of race and class. Journal of Gerontological Social Work, 28(1), 81-108.

Banzi, R., Camaioni, P., Tettamanti, M., Bertele, V., \& Lucca. U. (2016). Older patients are still under-represented in clinical trials of Alzheimer's disease. Alzheimer's Research \& Therapy, 8(32), 1-10.

Basit, T.M. (2003). Manual or electronic? The role of coding in qualitative data analysis. Educational Research, 45, 143-154.

Battistin, L. \& Cagnin, A. (2010). Vascular cognitive disorder: A biological and clinical overview. Neurochemical Research, 35(12), 1933-1938.

Beecher, H.K. (1966). Ethics and clinical research. New England Journal of Medicine, 274, 1354-1360.

Bekris, L.M., Yu, C.E., Bird, T.D., \& Tsuang, D.W. (2010). Genetics of Alzheimer disease. Journal of Geriatric Psychiatry \& Neurology, 23(4), 213-227. 
Bellah, R.N., Madsen, R., Sullivan, W.M., Swidler, A., \& Tipton, S.M. (1991). The good society. New York, NY: First Vintage Books Edition.

Benson, L., Harkavy, I., \& Puckett, J. (2011). Democratic transformation through university-assisted community schools. In Saltmarsh, J. \& Hartley, M. (Eds.). To serve a larger purpose: Engagement for democracy \& the transformation of higher education (pp. 49-81). Philadelphia, PA: Temple University Press.

Berwick, D.M., Nolan, T.W., \& Whittington, J. (2008). The Triple Aim: Care, health, and cost. Health Affairs, 27(3), 759-769.

Beyerlein, K. \& Bergstrand, K. (2016). It takes two: a dyadic model of recruitment to civic activity. Social Science Research, 60, 163-180.

Biogen. (2019, October 22). Biogen plans regulatory filing for aducanumab in Alzheimer's disease based on new analysis of larger dataaset from Phase 3 studies. [press release]. Retrieved from: https://investors.biogen.com/newsreleases/news-release-details/biogen-plans-regulatory-filing-aducanumabalzheimers-disease.

Black, B. S., Taylor, H. A., Rabins, P. V., \& Karlawish, J. (2018). Study partners perform essential tasks in dementia research and can experience burdens and benefits in this role. Dementia, 17(4), 494-514.

Blondell, S.J., Hammersley-Mather, R., \& Veerman, J.L. (2014). Does physical activity prevent cognitive decline and dementia?: A systematic review and meta-analysis of longitudinal studies. BMC Public Health, 14(510), 1-12.

Boller, F. \& Forbes, M.M. (1998). History of dementia and dementia in history: An overview. Journal of the Neurological Sciences, 158, 125-133.

Bonds, K. \& Lyons, K.S. (2017). Formal service use by African American individuals with dementia and their caregivers: An integrative review. Journal of Gerontological Nursing, 44(6), 33-39.

Botye, H. (2008). Against the current: Developing the civic agency of students. Change, $40(3), 8-15$.

Boustani, M., Peterson, B., Hanson, L., Harris, R., \& Lohr K.N. (2003) Screening for dementia in primary care: A summary of the evidence for the U.S. Preventive Services Task Force. Annals of Internal Medicine, 138(11), 927-37. 
Bowman Rogers, M. (2019, July 25). New PET staging scheme for amyloid? Alzforum. Retrieved from: https://www.alzforum.org/news/conference-coverage/new-petstaging-scheme-amyloid.

Boyer, E.L. (1996). The scholarship of engagement. Bulletin of the American Academy of Arts and Sciences, 49(7), 18-33.

Bradford, A., Kunik, M.E., Schultz, P., Williams, S.P., \& Singh, H. (2009). Missed and delayed diagnosis of dementia in primary care: Prevalence and contributing factors. Alzheimer's Disease \& Associated Disorders, 23(4), 306-14.

Brady, H.E., Schlozman, K.L., \& Verba, S. (1999). Prospecting for participants: Rational expectations and the recruitment of political activists. American Political Science Review, 93(1), 153-168.

Brown, R., Cadet, D., Houlihan, R., Thomson, M., Pratt, E., Sullivan, A. \& Siminoff, L. (2013). Perceptions of participation in a phase I, II, or III clinical trial among African American participants with cancer: What do refusers say? Journal of Oncology Practice, 9(6), 287-293.

Buchbinder, S.P., Metch, B., Holte, S.E., Scheer, S., Coletti, A., \& Vittinghoff, E. (2004). Determinants of enrollment in a preventive HIV vaccine trial: hypothetical versus actual willingness and barrier to participation. Journal of Acquired Immune Deficiency Syndrome, 36(1), 604-612.

Caldwell, K. (2014). Dyadic interviewing: A technique valuing the interdependence in interviews with people with intellectual disabilities. Qualitative Research, 14(4), 488-507.

Califf, R.M. (2006). Clinical trials bureaucracy: Unintended consequences of wellintentioned policy. Clinical Trials, 3(6), 496-502.

Campus Compact (2016). West Philadelphia Improvement Corps. Program Models. Retrieved from: https://compact.org/resource-posts/west-philadelphiaimprovement-corps/.

Canevelli, M., Valletta, M., Trebbastoni, A., Sarli, G., D’Antonio, F., Traciotti, L., ..., \& Bruno, G. (2016). Sundowning in dementia: Clinical relevance, pathophysiological determinants, and therapeutic approaches. Frontiers in Medicine, 3(73), 1-7.

Carder, P.C., Tunalilar, O., Elliott S., \& Dys, S. (2017). Oregon community-based care survey: assisted living, residential care, and memory care. Portland, OR: Portland State University. 
CareOregon. (2017). FoodRx: Capitalizing on the power of nutrition. Retrieved from: http://www.careoregon.org/about-us/what-we-do/community-programs/foodrx.

Carlisle, B., Kimmelman, J., Ramsay, T., \& MacKinnon, N. (2015). Unsuccessful trial accrual and human subject protections: An empirical analysis of recently closed trials. Clinical Trials, 12(1), 77-83.

Cary, M., Rubright, J., Grill, J., \& Karlawish, J. (2015). Why are spousal caregivers more prevalent than nonspousal caregivers as study partners in AD dementia clinical trials? Alzheimer's Disease and Associated Disorders, 29, 70-74.

Centers for Disease Control and Prevention. (1997). Principles of community engagement $\left(1^{\text {st }}\right.$ ed.). Atlanta, GA: CDC/ATSDR Committee on Community Engagement.

Centers for Medicare and Medicaid Services (2011). Program of All-Inclusive Care for the Elderly. Retrieved from: https://www.medicaid.gov/medicaid/ltss/pace/index.html.

Chandra, A., Miller, C.E., Acosta, J.D., Weiland, S., Trujillo, M., \& Plough, A. (2016). Drivers of health as a shared value: Mindset, expectation, sense of community, and civic engagement. Health Affairs, 35(11), 1959-1963.

Chen, R., Desai, N.R., Ross, J.S., Zhang, W., Chau, K.J., Wayda, B., ..., \& Krumholz, H.M. (2016). Publication and reporting of clinical trial results: Cross-sectional analysis across academic medical centers. BMJ, 352(8045), i637.

Chin, A.L., Negash, S., \& Hamilton, R. (2011). Diversity and disparity in dementia: The impact of ethnoracial differences in Alzheimer's disease. Alzheimer's Disease \& Associated Disorders, 25(3), 187-195.

ClinicalTrials.gov. (2019). A study to confirm safety and efficacy in BAN2401 in participants with early Alzheimer's disease (Clarity-AD). Retrieved from: https://clinicaltrials.gov/ct2/show/NCT03887455.

Colello, K.J. \& Talaga, S.R. (2015). Who pays for long-term care? A fact sheet. Congressional Research Service. Retrieved from: https://fas.org/sgp/crs/misc/R43483.pdf.

Collins, S., Klobuchar, A., Tillis, T., Whitehouse, S., Hoeven, J., Blumenthal, R., ..., \& King, Jr., A.S. (January 31, 2018). Letter to the President. Retrieved from: https://www.collins.senate.gov/sites/default/files/ALZ\%20funding\%20letter.pdf. 
Common Rule. 45 CFR 46 (2018). Retrieved from: https://www.ecfr.gov/cgi$\mathrm{bin} /$ retrieveECFR $\mathrm{gp}=\& \mathrm{SID}=83 \mathrm{~cd} 09 \mathrm{e} 1 \mathrm{c} 0 \mathrm{f5} 6637 \mathrm{~cd} 9 \mathrm{~d} 7513160 \mathrm{fc} 3 \mathrm{f} \& \mathrm{pitd}=20180$ $719 \& n=p t 45.1 .46 \& r=P A R T \& t y=H T M L \# s e 45.1 .46 \_1110$

Community Research Partners. (2012). Resources. Retrieved from: http://communityresearchpartners.net/.

Connell, C.M., Roberts, J.S., McLaughlin, S.J., \& Akinleye, D. (2009). Racial differences in knowledge and beliefs about Alzheimer disease. Alzheimer's Disease \& Associated Disorders, 23(2), 110-116.

Cook, K.E. (2008). In-depth interviews. In Given, L.M. (Ed.) The SAGE encyclopedia of qualitative research methods (p. 422). Thousand Oaks, CA: SAGE Publications Ltd.

Corbie-Smith, G., Thomas, S.B., Williams, M.V., Moody-Ayers, S. (1999). Attitudes and beliefs of African-Americans towards participation in medical research. Journal of General Internal Medicine, 14(9), 537-546.

Corriveau, R., Koroshetz, W., Gladman, J., Jeon, S., Babcock, D., Bennett, D., ..., \& Holtzman, D. (2017). Alzheimer's disease-related dementias summit 2016: National research priorities. Neurology, 89, 2381-2391.

Creswell, J.W. (2000). Determining validity in qualitative inquiry. Theory into Practice, $39,124-130$.

Creswell, J.W. (2013). Qualitative inquiry \& research design: Choosing among the five approaches. Thousand Oaks, CA: SAGE Publications, Inc.

Croff, R., Gowen, L.K., Lindauer, A., Shofner, S., Brown, K., \& Eckstrom, E. (in press). Including older rural adults in research: Practical guidelines for addressing the NIH Inclusion Across the Lifespan policy. Journal of Clinical and Translational Research.

Cummings, J., Lee, G., Mortsdorf, T., Ritter, A., \& Zhong, K. (2017). Alzheimer's disease drug development pipeline: 2017. Alzheimer's \& Dementia, 3(3), 367384.

Cummings, J.L., Morstorf, T., \& Zhong, K. (2014). Alzheimer's disease drug development pipeline: Few candidates, frequent failures. Alzheimer's Research \& Therapy, 6(37), 1-7. 
Danner, D.D., Darnell, K.R., \& McGuire, C. (2011). African American participation in Alzheimer's disease research that includes brain donation. American Journal of Alzheimer's Disease and Other Dementias, 26(6), 469-476.

De Strooper, B. (2018, January 11). Dementia is too big a problem to walk away fromfor Pfizer or any of us. The Guardian. Retrieved from: https://www.theguardian.com/commentisfree/2018/jan/11/dementia-pfizeralzheimers-research-big-pharma.

Delgado, R. \& Stefancic, J. (2001). Critical race theory: An introduction. New York: NYU Press.

Denhardt, J.V. \& Denhardt, R.B. (2003). The new public service: Service, not steering. Armonk, NY: M.E. Sharpe, Inc.

Department of Human Services Seniors and People with Disabilities Division. (2010). Oregon Administrative Rule: Chapter 411 Division 57 Memory Care Communities. Retrieved from: https://www.dhs.state.or.us/policy/spd/rules/411_057.pdf.

Dilts, D.M. \& Sandler, A.B. (2006). Invisible barriers to clinical trials: The impact of structural, infrastructural, and procedural barriers to opening oncology clinical trials. Journal of Clinical Oncology, 24(28), 4545-4552.

Dubowitz, T., Orleans, T., Nelson, C., May, L.W., Sloan, J.C. \& Chandra, A. (2016). Creating healthier, more equitable communities by improving governance and policy. Health Affairs, 35(11), 1970-1975.

Ehrlich, T. (2000). Preface. In Ehrlich, T. (Ed.). Civic responsibility and higher education. (pp. v-x). Westport, CT: The American Council on Education and The Oryx Press.

English, R., Lebovitz, Y., and Griffin, R. (2010). Transforming clinical research in the United States: Challenges and opportunities: workshop summary. Forum on Drug Discovery, Development, and Translation: Institute of Medicine. Retrieved from: www.nap.edu/catelog/12900/transformingclinical-research-in-the-united-stateschallenges-and-opportunities.

Esmail, L., Moore, E., \& Rein, A. (2015). Evaluating patient and stakeholder engagement in research: Moving from theory to practice. Journal of Comparative Effectiveness Research, 4(2), 133-145.

Espino, D.V. \& Lewis, R. (1998). Dementia in older minority populations. Issues of prevalence, diagnosis, and treatment. American Journal of Geriatric Psychiatry, 6(Suppl 1), S19-25. 
Estes, C.L. (1980). Constructions of reality. Journal of Social Issues, 36(2), 117-132.

European Science Foundation. (2009). Forward Look: Investigator-Driven Clinical Trials. Retrieved from:

http://archives.esf.org/fileadmin/Publicdocuments/Publications /IDCT.pdf.

European Science Foundation (2009). Forward look: Investigator-driven clinical trials.

Retrieved from:

http://archives.esf.org/fileadmin/Publicdocuments/Publications/IDCT.pdf.

Evans, D., Robertson, J. \& Candy, A. (2016). Use of photovoice with people with younger-onset dementia. Dementia, 15(4), 798-813.

Fagan, T. (2019, August 20). Move over, CSF, p-tau in blood also tells us there's plaque in the brain. Alzforum. Retrieved from:

https://www.alzforum.org/news/conference-coverage/move-over-csf-p-tau-bloodalso-tells-us-theres-plaque-brain.

Feldman, M.A., Bosett, J., Collet, C. \& Burnham-Roisa, P. (2014). Where are persons with intellectual disabilities in medical research? A survey of published clinical trials. Journal of Intellectual Disability Research, 58(9), 800-809.

Fernandopulle, R. (August 17, 2015). Breaking the fee-for-service addiction: Let's move to a comprehensive primary care payment model. Health Affairs Blog. Retrieved from: http://healthaffairs.org/blog/2015/08/17/breaking-the-fee-for-serviceaddiction-lets-move-to-a-comprehensive-primary-care-payment-model/.

Food \& Drug Administration. (2016). Human factors studies and related clinical study considerations in combination product design and development: Draft guidance for industry and FDA staff. Retrieved from:

https://www.fda.gov/downloads/RegulatoryInformation/Guidances/UCM484345. pdf.

Ford, C.L. \& Airhihenbuwa, C.O. (2010). Critical race theory, race equity, and public health: Towards antiracism praxis. American Journal of Public Health, 100(S1), S30-S35.

Foucault, M. (1980). Power/knowledge: Selected interviews and other writings, 19721977. C. Gordon (Ed.). New York, NY: Pantheon Books.

Freeman, R., Gwadz, M.V., Silverman, E., Kutnick, A., Leonard. N.R., Ritchie, A.S., ..., \& Martinez, B.Y. (2017). Critical race theory as a tool for understanding poor engagement along the HIV care continuum among African American/Black 
and Hispanic persons living with HIV in the United States: A qualitative exploration. International Journal for Equity in Health, 16(54), 1-14.

Freire, P. (1972). Pedagogy of the oppressed. New York, NY: Herder and Herder. (Originally published in 1968).

Frew, P.M., Archibald, M., Hixson, B., \& del Rio, C. (2011). Socioecological influences on community involvement in HIV vaccine research. Vaccine, 29(36), 6136-6143.

Friedman, D., Foster, C., Bergeron, C., Tanner, A., \& Kim, S. (2015). A qualitative study of recruitment barriers, motivators, and community-based strategies for increasing clinical trials participation among rural and urban populations. American Journal of Health Promotion, 29(5), 332-338.

Galbraith, J. (1973). Designing complex organizations. Reading, MA: Addison-Wesley.

Galvin, J.E., Howard, D.H., Denny, S.S., Dickinson, S., \& Tatton, N. (2017). The social and economic burden of frontotemporal degeneration. Neurology, 89(20), 20492056.

Gardner, R. \& Yaffe, K. (2014). Traumatic brain injury may increase risk of young onset dementia. Annals of Neurology, 75, 339-41.

Gates, B. (2017, November 13). Why I'm digging deep into Alzheimer's. [Blog post]. Retrieved from: https://www.gatesnotes.com/Health/Digging-Deep-IntoAlzheimers.

Gates, B. (2019, November 5). Here's a way you can help fight Alzheimer's. [Blog post]. Retrieved from: https://www.gatesnotes.com/Health/How-you-can-help-fightAlzheimers.

Geiling, N. (February 18, 2015). How Oregon's second-largest city vanished in a day. Smithsonian Magazine. Retrieved from:

https://www.smithsonianmag.com/history/vanport-oregon-how-countrys-largesthousing-project-vanished-day-180954040/?no-ist.

Gelman, C.R. \& Rhames, K. (2018). In their own words: The experiences and needs of children in younger-onset Alzheimer's disease and other dementia families. Dementia, 17(3), 337-358.

Gelmon, S., Bouranis, N., Sandberg, B., \& Petchel, S. (2018). Strategies for addressing the challenges of patient-centered medical home implementation: Lessons from Oregon. Journal of the American Board of Family Medicine, 31(3), 334-341. 
Gelmon, S., Wallace, N., Sandberg, B., Petchel, S., \& Bouranis, N. (2016). Implementation of Oregon's PCPCH program: Exemplary practice and program findings. Portland, OR: Portland State University.

Gifford, A.L., Cunningham, W.E., Heslin, K.C., Andersen, R.M., Nakazona, T., Lieu, D.K., ... , \& Bozzette, S.A. (2002). Participation in special research and access to experimental treatment by HIV-infected patients. New England Journal of Medicine, 346(18), 1373-1382.

Gilmore-Bykovskyi, A., Jin, Y., Gleason, C., Flowers-Benton, S., Block, L.M., DilworthAnderson, P., ..., \& Zuelsdorff, M. (2019). Recruitment and retention of underrepresented populations in Alzheimer's disease research: A systematic review. Alzheimer's and Dementia (NY), 19(5), 751-770.

Gitlin, L.N., Maslow K., \& Khillan R. (2018). Report to the National Advisory Council on Alzheimer's Research, Care and Services. National Research Summit on Care, Services, and Supports for Persons with Dementia and their Caregivers. Retrieved from: https://aspe.hhs.gov/system/files/pdf/259156/FinalReport.pdf.

Goldblatt, H., Karnieli-Miller., O. \& Neumann, M (2011). Sharing qualitative research findings with participants: Study experiences of methodological and ethical dilemmas. Patient Education and Counseling, 82, 389-95.

Goldman, J.S., Hahn, S.E., \& Bird T. (2011). Genetic counseling and testing for Alzheimer disease: Joint practice guidelines of the American College of Medical Genetics and the National Society of Genetic Counselors. Genetics in Medicine, 13(8), 597-605.

Gollhofer, S., Wiskemann, J., Schmidt, M., Klassen, O., Ulrich, C., Oelmann, ..., \& Steindorf, K. (2015). Factors influencing participation in a randomized controlled resistance exercise intervention study in breast cancer patients during radiotherapy. BMC Cancer, 15(186), DOI: 10.1186/s12885-015-1213-1.

Gomperts, S.N. (2016). Lewy-body dementias: Dementia with Lewy body and Parkinson disease dementia. Continuum (Minneapolis, Minnesota), 22(2), 435-463

Goodman, H. (2001). In-depth interviews. In Thyer, B.A. (Ed.). The handbook of social work research methods (pp. 308-319). Sage Publications Ltd; Thousand Oaks, CA.

Goss, K.A. (2010). Civil society and civic engagement: Towards a multi-level theory of policy feedbacks. Journal of Civil Society, 6(2), 119-143. 
Grill, J.D. \& Galvin, J.E. (2014). Facilitating Alzheimer's disease recruitment. Alzheimer's Disease \& Associated Disorders, 28(1), 1-8.

Habermas, J. (1974). Theory and practice (J. Viertel, Trans.). London, UK: Heinemann. (Original work published 1963).

Hannah-Jones, N. (May 10, 2011). Portland housing audit finds discrimination audit in 64 percent of tests; city has yet to act against landlords. The Oregonian. Retrieved from: https://www.oregonlive.com/portland/2011/05/a_portland_housing_audit_finds.ht $\mathrm{ml}$.

Hardman, R.J., Kennedy, G., Macpherson, H., Scholey, A.B., \& Pipingas, A. (2016). Adherence to a Mediterranean-style diet and effects on cognition in adults: A qualitative evaluation and systematic review of longitudinal and prospective trials. Frontiers in Nutrition, 3(22), 1-13.

Harris, D.A., Pensa, M.A., Redlich, C.A., Pisani, M.A., \& Rosenthal, M.S. (2016). Community-based participatory research is needed to address pulmonary health disparities. Annals of the American Thoracic Society, 13(8), 1231-1238.

Hawkes, N. (2018). Pfizer abandons research into Alzheimer's and Parkinson's diseases. BMJ, 360, k122.

Heller, C., Balls-Berry, J., Nery, J., Erwin, P., Littleton, D., Kim, M. \& Kuo, W. (2014). Strategies addressing barriers to clinical trial enrollment of underrepresented populations: a systematic review. Contemporary Clinical Trials, 39(2), 169-182.

Hemminki, E. (2016). Research ethics committees in the regulation of clinical trials: Comparison of Finland to England, Canada, and the United States. Health Research Policy and Systems, 14(5), 1-12.

Herbert, L.E., Weuve, J., Scherr, P.A., \& Evans D.A. (2013). Alzheimer disease in the United States (2010-2050) estimated using the 2010 census. Neurology, 80(19), $1178-1783$.

Hermann, L.K., Welter, E., Leverenz, J., Lerner, A.J., Udelson, N., Kanetsky, C., \& Sajatovic, M. (2018). A systematic review of dementia-related stigma research: Can we move the stigma dial? The American Journal of Geriatric Psychiatry, 26(3), 316-331.

Hernández, I., Fernández, M-V., Tàrraga, L., Boada, M., \& Ruiz, A. (2018).

Frontotemporal lobar degeneration: Review and update for clinical neurologists. Current Alzheimer's Research, 15, 1-24 
Hodes, R. (2018). We have a budget for FY 2019! National Institute on Aging. Retrieved from: https://www.nia.nih.gov/research/blog/2018/10/we-have-budget-fy-2019.

Holland, B.A. \& Gelmon, S.B. (1998). The state of the "engaged campus:" What have we learned about building and sustaining university-community partnerships. American Association of Higher Education Bulletin, October 1998, 3-6.

Holt, C. L., Graham-Phillips, A. L., Daniel Mullins, C., Slade, J. L., Savoy, A., \& Carter, R. (2017). Health ministry and activities in African American faith-based organizations: A qualitative examination of facilitators, barriers, and use of technology. Journal of Health Care for the Poor and Underserved, 28(1), 378388 .

Huang, H-H., \& Coker, A.D. (2010). Examining issues affecting African American participation in research studies. Journal of Black Studies, 40(4), 619-636.

Hudson, K., Lauer, M., \& Collins, F. (2016). Towards a new era of trust and transparency in clinical trials. Journal of the American Medical Association, 316(13), 13531354.

Hudson, R.B. (2014). Contemporary challenges to aging policy. In Hudson, R.B. (Ed.). The new politics of old age policy (3rd ed., pp. 3-19). Baltimore, MD: Johns Hopkins University Press.

Hudson, R.B. \& Gonyea, J.G. (2014). Baby Boomers and the political construction of old age. The Gerontologist, 52(2), 272-282.

Hurria, A., Dale, W., Mooney, M., Rowland, W.H., Ballman, K.V., Cohen, H.J., ..., \& the Cancer and Aging Research Group. (2014). Designing therapeutic clinical trials for older and frail adults with cancer: U13 conference recommendations. Journal of Clinical Oncology, 32(34), 2587-2594.

Iliffe, S., McGrath, T., \& Mitchell, D. (2013). The impact of patient and public involvement in the work of the Dementias and Neurodegenerative Diseases Research Network (DeNDRoN): Case studies. Health Expectations, 16(4), 351361.

Inungu, J.N., Bender, B. \& Gieber, L. (2017). Socio-behavioral challenges to HIV vaccine trials: Literature review. Clinical Research in HIV/AIDS: 4(1), 16d.

Israel, B.A., Litchenstein, R., Lantz, P.M., McGranaghan, R.J., Allen, A., Guzman. J.R., ..., \& Maciak, B. (2001). The Detroit Community-Academic Urban Research Center: Lessons learned in the development, implementation, and evaluation of a 
community-based participatory partnership. Journal of Public Health Management and Practice, 7(5), 1-19.

Israel, B.A., Schultz, A.J., Parker, E.A. Becker, A.B., Allen III, A.J., \& Guzman, J.R. (2003). Critical issues in developing and following CBPR principles. In Minkler, M. \& Wallerstein, N. (Eds). Community-based participatory research for health: From processes to outcomes (2nd ed., p. 56-73). San Francisco, CA: Jossey-Bass.

Jenkins, V. \& Fallowfield, L. (2015). For the benefit of others: Reasons why women with breast cancer participate in RCTs. Breast Care, 10, 88-93.

Johnson, D.A., Joosten, Y.A., Wilkens, C.H., \& Shibai, C.A. (2015). Case study: Community engagement and clinical trial success: Outreach to African American women. Clinical and Translational Science, 8(4), 388-390.

Johnson, J.M. \& Rowlands, T. (2012). The interpersonal dynamics of in-depth interviews. In Gubrium, J.F, Holstein, J.A., Marvasti, A.B. \& McKinney, K.D. (Eds.). The SAGE handbook of interview research: The complexity of the Craft ( $2^{\text {nd }}$ ed., pp. 99-114). SAGE Publications Ltd; Thousand Oaks, CA.

Jones, C.P. (2000). Levels of racism: A theoretical framework and a gardener's tale. American Journal of Public Health, 90(8), 1212-1215.

Jones, J. H. (1993). Bad blood: The Tuskegee syphilis experiment. New York: The Free Press.

Kaiser Family Foundation. (2016). An overview of Medicare. Retrieved from: http://files.kff.org/attachment/issue-brief-an-overview-of-medicare.

Kalaria, R.N., Akinyemi, R., \& Ihara, M. (2016). Stroke injury, cognitive impairment and vascular dementia. Biochimica et Biophysica Acta-Molecular Basis of Disease, 1862(5), 915-925.

Kalu, A.F. \& Bwalya, J.C. (2015). What makes qualitative research good research? An exploratory analysis of critical elements. International Journal of Social Science Research, 5(2), 43-56.

Karlawish, J., Kim, S., Knopman, D., Van Dyck, C., James, B., \& Marson D. (2008). The views of Alzheimer's disease patients and their study partners on proxy consent for clinical trial enrollment. American Journal of Geriatric Psychiatry, 16, 240247.

Karson, M. \& Goodwin, J. (2010). Critical thinking. In N. J. Salkind (Ed.), Encyclopedia of Research Design (p. 84). Thousand Oaks, CA: SAGE Publications, Inc. doi: 10.4135/9781412961288.n92. 
Katz, R.V., Green, B.L., Kressin, N.R., James, S.A., Wang, M.Q., ..., \& Russell, S.L. (2009). Exploring the "legacy" of the Tuskegee Syphilis Study: A follow-up study from the Tuskegee Legacy Project. Journal of the National Medical Association, 101(2), 179-183.

Kerr, C. (1994). Troubled times for American higher education. Albany: State University of New York Press.

Khachaturian, Z.S. (1998). An overview of Alzheimer's disease research. The American Journal of Medicine, 104(4, S1), 26S-31S.

King, W.D., Defreitas, D., Smith, K., Andersen, J., Perry, L.P., Adeyemi, T., ..., \& Mildvan, D. (2007). Attitudes and perceptions of AIDS clinical trials group site coordinators on HIV clinical trial recruitment and retention: A descriptive study. AIDS Patient Care \& STDS, 21(8), 551-563.

Knopman, D.S. \& Roberts, R.O. (2011). Estimating the number of persons with frontotemporal lobar degeneration in the US population. Journal of Molecular Neuroscience, 45(3), 330-335.

Kovera, M.B. (2010). Bias. Critical thinking. In N. J. Salkind (Ed.), Encyclopedia of Research Design (pp. 304-307). Thousand Oaks, CA: SAGE Publications, Inc. doi: 10.4135/9781412961288.n92.

Kramer, J., Smith, P., \& Califf, R. (2012). Impediments to clinical research in the United States. Clinical Pharmacology and Therapeutics, 93(3), 535-541.

Kremer, I. (2017). The imperative of diverse voices and collaborative decision-making. Opening Remarks at the 2017 NIH Research Summit on Dementia Care: Washington, DC.

Lang, L., Clifford, A., Wei, L., Zhang, D., Leung, D., Augustine, F., ..., \& Chen, R. (2017). Prevalence and determinants of undetected dementia in the community: A systematic literature review and a meta-analysis. BMJ Open, 7, 1-8.

Lang, R., Kelkar, V.A., Byrd, J.R., Edwards, C.L., Pericak-Vance, M., \& Byrd, G.S. (2013). African American participation in health-related research studies: Indicators for effective recruitment. Journal of Public Health Management and Practice, 19(2), 110-118.

Langer, E. (1964). Human experimentation: Cancer studies at Sloane-Kettering stir public debate on medical ethics. Science, 143, 551-553. 
Lapadat, J.C. (2010). Thematic Analysis. In: Mills, A.J., Durepose, G., Wiebe, E. (Eds.). Encyclopedia of case study research, Vol 2. (pp. 925-927). Thousand Oaks, CA: SAGE Publications.

Lauer, M. (November 15, 2017). Teaming with ORCID to reduce burden and improve transparency. [Blog post]. Retrieved from:

https://nexus.od.nih.gov/all/2017/11/15/teaming-with-orcid-to-reduce-burdenand-improve-transparency/.

Lautenschlager, N.T., Cupples, L.A., Rao, V.S., Auerbach, S.A., Becker, R., Burke, J., ..., \& Farrer, L.A. (1996). Risk of dementia among relatives of Alzheimer's disease patients in the MIRAGE study: What is in store for the oldest old? Neurology, 46(3), 641-650.

Lavis, J.N., Robertson, D., Woodside, J.M., McLeod, C.B., Abelson, J., \& the Knowledge Transfer Group. (2003). How can research organizations more effectively transfer knowledge to decision makers? The Milbank Quarterly, 81(2), 221-248.

Law, E. Russ, T.C. \& Connolly, P.J. (2014). What motivates patients and carers to participate in dementia research? Results from a series of focus groups in Scotland. International Journal of Geriatric Psychiatry, 29, 106-107.

Lawrence, P.R. \& Lorsch, J.W. (1967). Organization and environment: Managing differentiation and integration. Boston, MA: Harvard University.

Leaders Engaged in Alzheimer's Disease (LEAD) Coalition (2017). Who We Are. Retrieved from: http://www.leadcoalition.org/who-we-are/ourmembers/alzheimers-orange-county/.

Lee, L., Piliavan, J.A., \& Call, V.R.A. (1999). Giving time, money, and blood: Similarities and differences. Social Psychology Quarterly, 62(3), 276-290.

Lennox, N., Taylor, M., Rey-Conde, T., Bain, C., Purdie, D.M., \& Boyle, F. (2005). Beating the barriers: recruitment of people with intellectual disability to participate in research. Journal of Intellectual Disability Research, 49(P4), 296305.

Levin, J.S. (1986). Roles for the black pastor in preventive medicine. Pastoral Psychology, 35(2), 94-103.

Lewy Body Dementia Association (2015). Treatment of Lewy Body Dementia. Retrieved from: https://www.lbda.org/sites/default/files/treatment.pdf 
LiveWell. (2018). Empowering partners: Engaging individuals with dementia and care partners in PCOR/CER. [Powerpoint slides]. Retrieved from:

https://www.pcori.org/sites/default/files/Empowering-Partners-Presentation.pdf.

Livingston, G., Sommerlad, A., Orgeta, V., Costafreda, S.G., Huntley, J., Ames, D., ..., \& Mukadam, N. (2017). Dementia prevalence, intervention, and care. The Lancet, 390, 2673-2674.

Lounsbury, M. \& Pollack, S. (2001). Institutionalizing civic engagement: Shifting logics and the cultural repackaging of service-learning in US higher education.

Organization, 8(2), 319-339.

Love, S. (2005). Neuropathological investigation of dementia: A guide for neurologists. Journal of Neurology, Neurosurgery, \& Psychiatry, 76(S5), v8-v14.

Luzi, A.M., Gallo, P., Colucci, A., Marcotullio, S., Bellino, S., Longo, O., \& Ensoli, B. (2011). Communication, recruitment, and enrollment in the preventative and therapeutic Phase I clinical trial against HIV/AIDS based on the recombinant HIV-1 tat protein. AIDS Care, 23(8), 939-946.

Mahley, R.W. \& Rall Jr., S.C. (2000). Apolipoprotein E: Far more than a lipid transport protein. Annual Review of Genomics and Human Genetics, 1, 507-37.

Mahon, E., Roberts, J., Furlong, P., Uhlenbrauck, G., \& Bull, J. (2016). Barriers to clinical trial recruitment and possible solutions. Applied Clinical Trials, 25(2), 2025.

Martin, T.L., Plough, A., Carman, K.G., Leviton, L., Bogdan, O. \& Miller, C.E. (2016). Strengthening integration of health services and systems. Health Affairs, 35(11), 1976-1981.

Masliah, E. (March 7, 2018). Seeking your ideas for ways to enhance recruitment and retention of Alzheimer's disease study participants. Retrieved from:

https://www.nia.nih.gov/research/blog/2018/03/seeking-your-ideas-waysenhance-recruitment-and-retention-alzheimers-disease.

McCaffrey, P. (2019, August 30). Technology brings dementia detection to the home. Alzforum. Retrieved from: https://www.alzforum.org/news/conferencecoverage/technology-brings-dementia-detection-home.

McCullough, J.M. \& Leider, J.P. (2016). Government spending in health and nonhealth sectors associated with improvement in county health rankings. Health Affairs (Millwood), 35(11), 2037-43. 
McDonald, K.E. \& Stack, E. (2016). You say you want a revolution: An empirical study of community-based participatory research with people with developmental disabilities. Disability \& Health Journal, 9(2), 201-207.

McDonald, K.E., Conroy, N.E., Kim, C.I., LoBraico, E.J., Prather, E.M., \& Olick, R.S. (2016). Is safety in the eye of the beholder? Safeguards in research with adults with intellectual disability. Journal of Empirical Research of Human Research Ethics, 11(5), 424-438.

McKeith, I.G., Boeve, B.F., Dickson, D.W., Halliday, G., Taylor, J.P., Weintraub, D., ..., \& Kosaka, K. (2017). Diagnosis and management of dementia with Lewy bodies. Neurology, 89(1), 88-100.

Meador, K.J. (2015). Decline of clinical research in academic medical centers. Neurology, 85(13), 1171-1176.

Mendez-Luck, C.A. Luck, J., Larson, A.E., \& Dyer, G.B. (2017). The state of nursing facilities in Oregon, 2016. Corvallis, OR: OSU College of Public Health and Human Sciences.

Merck \& Co. (2018). Merck announces discontinuation of APECS study evaluating Verubecestat (MK-8931) for the treatment of people with prodromal Alzheimer's Disease. Retrieved from: https://investors.merck.com/news/press-releasedetails/2018/Merck-Announces-Discontinuation-of-APECS-Study-EvaluatingVerubecestat-MK-8931-for-the-Treatment-of-People-with-ProdromalAlzheimers-Disease/default.aspx.

Mergenthaler, P., Lindauer, U., Dienel, G.A., \& Meisel, A. (2013). Sugar for the brain: The role of glucose in physiological and pathological brain function. Trends in Neuroscience, 36(10), 587-97.

Mettler, S. \& Welch, E. (2004). Civic generation: Policy feedback effects of the G.I. Bill on political involvement over the life course. British Journal of Political Science, 34(3), 497-518.

Mettler, S. (2002). Bringing the state back in to civic engagement: Policy feedback effects of the GI Bill for World War II veterans. The American Political Science Review, 96(2), 351-365.

Michener, L., Cook, J., Ahmend, S.M., Yonas, M.A., Coyne-Beasley, J., \& AguilarGaxiola, S. (2012). Aligning the goals of community-engaged research: Why and how academic health centers can successfully engage with communities to improve health. Academic Medicine, 87(3), 285-291. 
Miles, M.B., Huberman, A.M., \& Saldana, J. (2020). Qualitative Data Analysis: A Methods Sourcebook (4 ${ }^{\text {th }}$ Ed.). Thousand Oaks, California: SAGE Publications.

Millinaar, J.K., Bakker, C., Koopmans, R.T., Verkey, F.R., Kurz, A. \& de Vugt, M.E. (2016). The care needs and experiences with the use of services of people with young-onset dementia and their caregivers: A systematic review. International Journal of Geriatric Psychiatry, 31(12), 1261-1276.

Minkler, M. \& Wallerstein, N. (2008). Introduction to community-based participatory research: New issues and emphases. In Minkler, M. and Wallerstein, N. (Eds). Community-based participatory research for health: From processes to outcomes $\left(2^{\text {nd }}\right.$ ed., pp. 5-24). San Francisco, CA: Jossey-Bass.

Mintzberg, H. (1983). Structure in fives: Designing effective organizations. Englewood Cliffs, NJ: Prentice-Hall, Inc.

Monson, K., Parlour, L., Simcock, R., Fallowfield, L. \& Jenkins, V. (2012). Group recruitment sessions enhance patient understanding in a small multi-centre phase III clinical trial. Contemporary Clinical Trials, 33(2), 286-290.

Moody, H.R. (2004). Silver industries and the new aging enterprise. Generations, 75-79. Morgan, D. (2008). Snowball sampling. In Given, L.M. (Ed.). The SAGE encyclopedia of qualitative research methods (pp. 816-828). Thousand Oaks, California: SAGE Publications.

Morgan, D.L., Ataie, J., Carder, P., \& Hoffman, K. (2013). Introducing dyadic interviews as a method for collecting qualitative data. Qualitative Health Research, 23(9), 1276-1284.

Morris, M.C., Tangney, C.C., Wang, Y., Sacks, F.M., Barnes, L.L., Bennett, D.A., \& Aggarwal, N.T. (2015). MIND diet slows cognitive decline with aging. Alzheimer's \& Dementia, 11(9), 1015-22.

Multnomah County Health Department (2014). 2014 Report Card on Racial and Ethnic Disparities: December 2014). Retrieved from: https://multco.us/file/37530/download.

Nabers, A., Perna, L., Lange, J., Mons, U., Schartner, J., Guldenhaupt, J., ..., \& Brenner, H. (2018). Amyloid blood biomarker detects Alzheimer's disease. EMBO Molecular Medicine, e8763.

Nathan, D.G. \& Wilson, J.D. (2003). Clinical research and the NIH - A report card. New England Journal of Medicine, 349, 1860-1865. 
National Alliance for Caregiving \& AARP Public Policy Institute (2015). Caregiving in the USA. Retrieved from:

https://www.aarp.org/content/dam/aarp/ppi/2015/caregiving-in-the-united-states2015-report-revised.pdf.

National Commission for the Protection of Human Subjects of Biomedical and Behavioral Research. (1979). The Belmont report: Ethical principles and guidelines for the protection of human subjects in research. Retrieved from: https://www.hhs.gov /ohrp/regulations-and-policy/belmont-report/read-thebelmont-report/index.html.

National Institute on Aging (2017). Video: How Alzheimer's changes the brain. Retrieved from: https://www.nia.nih.gov/health/video-how-alzheimers-changesbrain.

National Institute on Aging. (2018). Together we make the difference: National strategy for recruitment and participation in Alzheimer's and related dementias clinical research. Retrieved from: https://www.nia.nih.gov/sites/default/files/ 201810/alzheimers-disease-recruitment-strategy-final.pdf.

National Institutes of Health (1994). NOT-OD-00-048: NIH Guidelines on the Inclusion of Women and Minorities as Subjects in Clinical Research. Retrieved from: https://grants.nih.gov/grants/guide/notice-files/not94-100.html.

National Institutes of Health (2000). NOT-OD-00-048: NIH Guidelines on the Inclusion of Women and Minorities as Subjects in Clinical Research-Updated August 2, 2000. Retrieved from: https://grants.nih.gov/grants/guide/notice-files/NOT-OD00-048.html.

National Institutes of Health (2014). NOT-OD-15-105: Notice of Revised NIH Definition of "Clinical Trial." Retrieved from: https://grants.nih.gov/grants/guide/noticefiles/NOT-OD-15-015.html.

National Institutes of Health (2016a). NOT-OD-16-094: Final NIH Policy on the Use of a Single Institutional Review Board for Multi-site Research. Retrieved from: https:/grants.nih.gov/grants/guide/notice-files/NOT-OD-16-094.html.

National Institutes of Health (2016b). NOT-OD-16-148: Policy on Good Clinical Practice Training for NIH Awardees Involved in NIH-funded Clinical Trials. Retrieved from: https://grants.nih.gov/grants/guide/notice-files/NOT-OD-16148.html. 
National Institutes of Health (2016c). NOT-OD-16-149. NIH Policy on the Dissemination of NIH-Funded Clinical Trial Information. Retrieved from:

https://grants.nih.gov/grants/guide/notice-files/not-od-16-149.html.

National Institutes of Health (2017a). NOT-OD-18-116: Revision: NIH Policy and Guidelines on the Inclusion of Individuals Across the Lifespan as Participants in Research Involving Human Subjects. Retrieved from: https:/grants.nih.gov/grants/guide/notice-files/NOT-OD-18-116.html.

National Institutes of Health (2017b). NIH's Definition of a Clinical Trial. Retrieved from: https://grants.nih.gov/policy/clinical-trials/definition.htm.

National Institutes of Health (2017c). NOT-OD-18-014: Amendment: NIH Policy and Guidelines on the Inclusion of Women and Minorities as Subjects in Clinical Research. Retrieved from: https:/grants.nih.gov/grants/guide/notice-files/NOTOD-18-014.html.

National Institutes of Health (2017d). NOT-OD-17-109: Notice of Changes to NIH Policy for Issuing Certificates of Confidentiality. Retrieved from: https:/grants.nih.gov/grants/guide/notice-files/NOT-OD-17-109.html.

National Institutes of Health (2018). PAR-18-749: Examining Diversity, Recruitment and Retention in Aging Projects (R24 Clinical Trials Not Allowed). Retrieved from: https://grants.nih.gov/grants/guide/pa-files/PAR-18-749.html.

National Institutes of Health (2019). NOT-OD-20-044. Request for Information (RFI): Invitation to Comment on Inclusion Across the Lifespan II Workshop. Retrieved from: https://grants.nih.gov/grants/guide/notice-files/NOT-OD-20-044.html.

Neary, D., Snowden, J.S., Northen, B., \& Goulding, P. (1988). Dementia of frontal lobe type. Journal of Neurology, Neurosurgery, and Psychiatry, 51(3), 353-361.

Newington, L. \& Metcalfe, A. (2014). Researchers' and clinicians' perceptions of recruiting participants to clinical research: A thematic meta-synthesis. Journal of Clinical Medical Research, 6(3), 162-172.

Newman, S.D., Andrews, J.O., Magwood, G.S., Jenkins, C., Cox, M.J. \& Willliamson, D.C. (2011). Community advisory boards in community-based participatory research: A synthesis of best processes. Preventing Chronic Disease, 8(3), 1-12.

Nicolaidis, C., Raymaker, D., Katz, M., Oschwald, M., Goe, R., Leotti, S., ..., \& Partnering with People with Disabilities to Address Violence Consortium. (2015). Community-based participatory research to adapt health measures for use by people with developmental disabilities. Progress in Community Health Partnerships, Research, Education, Action, 9(2), 157-170. 
Nicolaidis, C., Raymaker, D., McDonald, K.E., Dern, S., Ashkenazy, E., Robertson, S., \& Baggs, A. (2011). Collaboration strategies in nontraditional community-based participatory research partnerships: Lessons from an academic-community partnership with autistic self-advocates. Progress in Community Health Partnerships, Research, Education, Action, 5(2), 143-150.

NORC at the University of Chicago. (2014). ACA Section 2401, Community First Choice Option (Section 1915(k) of the Social Security Act): Oregon State Option (Section 1915(k) of the Social Security Act): Oregon State Plan Amendment Summary. Research Summary. Retrieved from: https://www.medicaid.gov/medicaid/hcbs/downloads/or-cfc-spamatrix.pdf;/hcbs/downloads/or-cfc-spa-matrix.pdf.

Norton, M.C., Smith, K.R., Ostbye, T., Tschnatz, J.T., Corcoran, C., Schwartz, S., ..., \& Welsh Bohmer, K.A. (2010). Greater risk of dementia when spouse has dementia? The Cache County study. Journal of the American Geriatric Society, 58(5), 895900 .

Nuremberg Code. (1947). Retrieved from: https://history.nih.gov/research/downloads/nuremberg.pdf.

Office for Human Research Protections. (2017). Informed Consent FAQ. Retrieved from: https://www.hhs.gov/ohrp/regulations-and-policy/guidance/faq/informedconsent/index.html.

Office for Human Research Protections. (n.d.). Office for Human Research Protections. Retrieved from: https://www.hhs.gov/ohrp/.

OHSU Brain Institute (2018). Patient Education and Resources. Retrieved from: https://www.ohsu.edu/xd/health/services/brain/gettingtreatment/diagnosis/alzheimers-aging-dementia/education-andresources/index.cfm.

Oregon Department of Human Services (2015). Family Caregiver Support Program Standards. Retrieved from: http://www.oregon.gov/DHS/SENIORSDISABILITIES/SUA/Documents/FCSP\%20Standards\%202015\%20Revised\%20 2-26- 16.pdf.

Oregon Department of Human Services. (2017). OAR Chapter 411 Division 32: Oregon Project Independence. Retrieved from:

http://www.dhs.state.or.us/policy/spd/rules/411_032.pdf. 
Oregon Health Authority. (n.d.). Coordinated Care Organizations (CCOs). Retrieved from: https://www.oregon.gov/oha/HSD/OHP/Pages/Coordinated-CareOrganizations.aspx.

Oregon State Legislature (2017). HB 3359. Retrieved from: https://olis.leg.state.or.us/liz/2017R1/Downloads/MeasureDocument/HB3359.

Owens, O.L., Jackson, D.D., Thomas, T.L., Friedman, D.B., \& Hebert, J.R. (2013). African American men's and women's perceptions of clinical trial research: focusing on prostate cancer among a high-risk population in the South. Journal of Health Care for the Poor and Underserved, 24(4), 1-16.

Palombaro, K.M., Black, J.D., Dole, R.L., Pierce, J.L., Santiago, M.R. \& Sabara, E.J. (2017). Assessing the development of civic mindedness in a cohort of physical therapy students. Journal of the Scholarship of Teaching and Learning, 17(4), 3143.

Parks, C. (2012, September 22). Fifty years later, Emanuel Legacy Medical Center apologizes attempts to make amends for razing neighborhoods. The Oregonian Retrieved from: https://www.oregonlive.com/portland/2012/09/post_273.html.

Patient-Centered Outcomes Research Institute. (2013). Strategic Plan. Retrieved from: https://www.pcori.org/sites/default/files/PCORI-Strategic-Plan.pdf.

Patient-Centered Outcomes Research Institute (2017). PCORI Methodology Report: June 2017. Retrieved from: https://www.pcori.org/sites/default/files/PCORIMethodology-Report.pdf.

Patient-Centered Outcomes Research Institute. (2019). Empowering partners: Engaging individuals with dementia and care partners in PCOR/CER. PCORI. Retrieved from: https://www.pcori.org/research-results/2018/empowering-partnersengaging-individuals-dementia-and-care-partners-pcorcer.

Patient-Centered Outcomes Research Institute (2020a). Dementia and Cognitive Impairment. Retrieved from: https://www.pcori.org/topics/dementia-andcognitive-impairment.

Patient-Centered Outcomes Research Institute (2020b). Cancer. Retrieved from: https://www.pcori.org/topics/cancer.

Patient-Centered Outcomes Research Institute (2020c). Mental and Behavioral Health. Retrieved from: https://www.pcori.org/researchresults?f\%5B0\%5D=field_project_type $\% 3 \mathrm{~A} 298 \& \mathrm{f} \% 5 \mathrm{~B} 1 \% 5 \mathrm{D}=$ field_award_tags $\% 3 \mathrm{~A} 234$ 
Penberthy, L., Brown, R., Wilson-Genderson, M., Dahman, B., Ginder, G., \& Siminoff, L. (2012). Barriers to therapeutic clinical trials enrollment: Differences between African-American and White cancer patients identified at the time of eligibility assessment. Clinical Trials, 9(9), 788-797.

Penn Frontotemporal Degeneration Center. (n.d.). Common Medications and Treatments. Retrieved from: https://ftd.med.upenn.edu/living-with-ftd-relateddisorders/common-medications-treatments.

Perez-Stable, E.J. (2018, June 28). Communicating the value of race and ethnicity in research. Science, Trust, and Public Health. Retrieved from: https://www.nih.gov/about-nih/what-we-do/science-health-publictrust/perspectives/science-health-public-trust/communicating-value-raceethnicity-research.

Pickett, J. (2017). European perspectives and experiences in involving persons with dementia and dementia family caregivers as members of the research team. [Powerpoint presentation]. 2017 NIH Research Summit on Dementia Care. Washington, DC.

Pickett, J. (2018). Pfizer ends neuroscience discovery programme, including research into Alzheimer's disease treatment- Alzheimer's Society comment. The Alzheimer's Society. Retrieved from:

https://www.alzheimers.org.uk/news/article/292/pfizer_ends_neuroscience_disco very_programme_including_research_into_alzheimer_s_disease_treatment_\%E2 \%80\%93_alzheimer_s_society_comment.

Pierson, P. (1993). When effect becomes cause: Policy feedback and political change. World Politics, 45(4), 595-628.

Plassman, B.L., Langa, K.M., Fisher, G.G., Heeringa, S.G., Weir, D.R., Ofstedal, M.B., ..., \& Wallace, R.B. (2007). Prevalence of dementia in the United States: The aging, demographics, and memory study. Neuroepidemiology, 29(1-2), 125-132.

Plunkett, R. \& Chen, P. (2016). Supporting healthy dementia culture: An exploratory of the church. Journal of Religious Health, 55, 1917-1928.

Pool, L.R., Weuve, J., Wilson, R.S., Buotmann, U., Evans, D.A., \& Mendes de Leon, C.F. (2016). Occupational cognitive requirements and late-life cognitive aging. Neurology, 86(15), 1386-92.

Potter, M., Handley, M., Goldstein, E., Abrams, D., Alvarez, R., Benson, M., ..., \& Raine-Bennett, T. (2010). Community-engaged research: A quick-start guide for 298 
community-based clinicians. San Francisco, CA: Clinical Translational Science Institution Community Engagement Program.

PreSERVE Coalition for Black/African American Memory and Brain Health (n.d.). Who we are. Retrieved from: http://preservecoalition.org/.

Prince, M., Wimo, A., Guerchet, M., Ali, G.C., Wu, Y.T., Prina, M. \& Alzheimer's Disease International. (2015). World Alzheimer's report 2015, the global impact of dementia: An Analysis of prevalence, incidence, cost, and trends. London, UK: Alzheimer's Disease International. Retrieved from: https://www.alz.co.uk/research/WorldAlzheimerReport2015.pdf

Putnam, R.D. (2000). Bowling alone: The collapse and revival of American community. New York, New York: Simon \& Schuster.

Rabinovici, G.D., Gatsonis, C., Apgar, C., Chaudhary, K., Gareen, I., Hanna, L., ..., \& Carillo, M. (2019). Association of amyloid Positron Emission Tomography with subsequent change in clinical management among Medicare beneficiaries with mild cognitive impairment or dementia. Journal of the American Medical Association, 321(13), 1286-1294.

Rahman, S., Majumder, M.A.A., Shaba, S.F., Rahman, N., Ahmed, M., Abdulrahman, K.B. \& D'Souza, U.J.A. (2011). Physician participation in clinical research and trials: issues and approaches. Advances in Medical Education \& Practice, 2, 8593.

Readhead, B.,Haure-Mirande, J.V., Funk, C.C., Richards, M.A., Shannon, P., Harotunian, V., ..., \& Dudley, J.T. (2018). Multiscale analysis of independent Alzheimer's cohorts finds disruption of molecular, genetic, and clinical networks by human herpesvirus. Neuron, 99(1), 64-82.

Rice, T.W. (2008). The historical, ethical, and legal background of human-subjects research. Respiratory Care, 53(10), 1325-1329.

Richesson, R.L. \& Krischner, J. (2007). Data standards in clinical research: Gaps, overlaps, challenges, and future directions. Journal of the American Medical Informatics Association, 14, 687-696.

Robert Wood Johnson Foundation. (2016). The Culture of Health Action Framework. Retrieved from: https://www.rwjf.org/en/cultureofhealth.html.

Roberts, J.S., Connell, C.M., Cisewski, D., Hipps, Y.G., Demissie, S., \& Green, R.C. (2003). Differences between African Americans and whites in their perceptions of Alzheimer disease. Alzheimer Disease \& Associated Disorders, 17(1), 1926. 
Roberts, R. \& Knopman, D.S. (2013). Classification and epidemiology of MCI. Clinical Geriatric Medicine, 29(4), 1-19.

Rollin-Sillaire, A., Breuilh, L., Salleron, J., Bombois, S., Cassagnaud, P., Deramecourt, V., Mackowiack, M.A., \& Pasquier, F. (2013). Reasons that prevent the inclusion of Alzheimer's disease patients in clinical trials. British Journal of Clinical Pharmacology, 75(4), 1089-1097.

Sa, M.S., Li, S.X., \& Faubert, M. (2011). Faculties of education and institutional strategies for knowledge mobilization: an exploratory study. Higher Education, $61,501-\quad 512$.

Saltmarsh, J. \& Hartley, M. (2011). Democratic engagement. In Saltmarsh, J. \& Harley, M. (Eds.) To serve a larger purpose: Engagement for democracy and the transformation of higher education. (pp. 14-26). Philadelphia, PA: Temple University Press.

Sanders, T.H. \& Putnam, R.D. (2010). Still bowling alone? The post-9/11 split. Journal of Democracy, 21(1), 9-16.

Sando, S.B., Melquist, S., Cannon,A., Hutton, M., Sletvold, O., Saltvedt, I., ..., \& Aasly, J. (2008). Risk-reducing effect of education in Alzheimer's disease. International Journal of Geriatric Psychiatry, 23(11), 1156-1162.

Schneider, A.L., Ingram, H., \& deLeon, P. (2014). Democratic policy design: Social construction of target populations. In Sabatier, P.A \& Weible, C.M. (Eds.) Theories of the policy process (3rd ed., pp. 105-149). Boulder, CO: Westview Press.

Schneider, J.A., Arvanitakis, Z., Bang, W., \& Bennett, D.A. (2007). Mixed brain pathologies account for most dementia cases in community-dwelling older persons. Neurology, 69(24), 2197-2204.

Schneider, L.S. (2010). The potential and limitations for clinical trials in early-onset Alzheimer's disease and some recommendations. The Journal of Nutrition, Health, and Aging 14(4), 295-298.

Schneider, L.S., Olin J., Lyness, S., \& Chu, H. (1997). Eligibility of Alzheimer's disease clinic patients for clinical trials. Journal of the American Geriatric Society, 45, 923-928.

Scott, W.R. (1987). Organizations: Rational, natural, and open systems (2nd ed.). Englewood Cliffs, NJ: Prentice-Hall, Inc. 
Selby, J.V., Beal, A.C., Frank, L. (2012) The Patient-Centered Outcomes Research Institute (PCORI) national priorities for research and initial research agenda. Journal of the American Medical Association, 307(15), 1583-1584.

Shavres, V.L., Lynch, C.F., \& Burmeister, L.F. (200). Knowledge of the Tuskegee study and its impact on the willingness to participate in medical research studies. Journal of the National Medical Association, 91, 563-572.

Skocpol, T. (1992). Protecting soldiers and mothers: The political origins of social policy in the United States. Cambridge, MA \& London, UK: The Belknap Press of Harvard University Press.

Smith, J. \& Merrithew, N. (2017). Primary care as a cornerstone of reform. In Stock, R. \& Goldberg, B. (Eds.). Health reform policy to practice: Oregon's path to a sustainable health system: A study in innovation. (pp. 85-99). London, UK: Elsevier.

Snowden, J.S., Neary, D., \& Mann, D.M.A. (2002). Frontotemporal dementia. British Journal of Psychiatry, 180(2), 140-143.

Sojka, B.N. \& Sojka, P. (2008). The blood donation experience: self-reported motives and obstacles for donating blood. Vox Sanguinis, 94, 56-63.

Sood, A., Prasad, K., Chhatwani, L., Shinozaki, E., Cha, S.S., Loehner, L.L. \& WahnerRoedler, D.L. (2009). Patient's attitudes and preferences about participation and recruitment strategies in clinical trials and recruitment strategies in clinical trials. Mayo Clinic Proceedings, 84(3), 243-247.

Stephenson J. (2001). Racial barriers may hamper diagnosis, care of patients with Alzheimer disease. Journal of the American Medical Association, 286, 779-780.

Stone, R. (2011). Long-term care for the elderly. Washington, DC: The Urban Institute Press.

Stout, S.S., Powers, L., Ramsey, R., Rickter J., Ewanchyna, K., \& Dillon, K. (2017). The healthcare journey to population health: Guideposts from the Oregon experience. In Stock, R. \& Goldberg, B. (Eds.). Health reform policy to practice: Oregon's path to a sustainable health system: A study in innovation. (pp. 283-299). London, UK: Elsevier.

Strobel, G. (n.d.). What is early-onset familial AD? Alzforum. Retrieved from: https://www.alzforum.org/early-onset-familial-ad/overview/what-early-onsetfamilial-alzheimer-disease-efad. 
Sugarman, J., Getz, K., Speckman, J.L., Byne, M.M. \& Emanuel, E.J. (2005). Consortium to evaluate clinical research ethics: The cost of institutional review boards in academic medical centers. New England Journal of Medicine, 352, 1825-1827.

Swanson C.J., Zhang, Y., Dhadda, S., Wang, J., Kaplow, J., Lai, R., ..., \& Luthman, J. (2018). Treatment of early AD subjects with BAN2401, an anti-A $\beta$ protofibril monoclonal antibody, significantly clears amyloid plaque and reduces clinical decline. [Plenary session] Alzheimer's Association International Conference. Chicago, IL.

Tanner, D. (2012). Co-research with people with dementia: Experiences and reflections. Journal of Mental Health, 21(3), 296-306.

Tillman, L. (2017). Promoting health equity. In Stock, R. \& Goldberg, B. (Eds.). Health reform policy to practice: Oregon's path to a sustainable health system: A study in innovation. (pp.153-171). London, UK: Elsevier.

Towe, V.L., Leviton, L., Chandra, A., Sloan. J.C., Tait, M. \& Orleans, T. (2016). Crosssector collaborations and partnerships: Essential ingredients to help shape health and wellbeing. Health Affairs, 35(11), 1964-1969.

Trzepacz, P.T., Hochstetler, H., Wang, S., Walker, B., Saykin, A.J., \& the Alzheimer's Disease Neuroimaging Initative. (2015). Relationship between the Montreal Cognitive Assessment and Mini-mental State Examination for assessment of mild cognitive impairment in older adults. BMC Geriatrics, 15(107), 1-9.

U.S. Census Bureau (2019). 2012-2016 American Community Survey 5-Year Estimates. Retrieved from: https://www.census.gov/programs-surveys/acs/technicaldocumentation/table-and-geography-changes/2016/5-year.html.

U.S. Food \& Drug Administration (2018). The drug development process. Retrieved from: https://www.fda.gov/forpatients/approvals/drugs/ucm405622.htm.

United States Census Bureau. (2018). Population estimates, July 1, 2018, (V2018). QuickFacts Oregon. Retrieved from: https://www.census.gov/quickfacts/OR.

University of Washington (2018). Community Events and Programs. Retrieved from: http://depts.washington.edu/mbwc/events/community-events-programs.

UyBico, S.J., Pavel, S., \& Gross, C.P. (2007). Recruiting vulnerable populations into research: A systematic review of recruitment interventions. Journal of General Internal Medicine, 22(6), 852-863. 
Vigoda-Gadot, E. \& Cohen. A. (2004). Citizenship and management in public administration: Integrating behavioral theories and managerial thinking. Cheltenham, UK: Edward Elgar Publishing Limited.

Villemagne, V.L., Burnham, S., Brown, B., Ellis, K.A., Salvado, O., Szoeke, C., ..., \& the Australian Imaging Biomarkers and Lifestyle (AIBL) Research Group. (2013). Amyloid beta deposition, neurodegeneration, and cognitive decline in sporadic Alzheimer's disease: A prospective cohort study. The Lancet Neurology, 12(4), 357-367.

Voytek, C.D., Jones, K.T. \& Metzger, D.S. (2011). Selectively willing and conditionally able: HIV vaccine trial participation among women at "high risk" of HIV infection. Vaccine, 29(36), 6130-6135.

Wallerstein, N. \& Duran, B. (2008). The theoretical, historical, and practice roots of CBPR. In Minkler, M. \& Wallerstein, N. (Eds.), Participatory research: From processes to outcomes. (pp. 25-46). San Francisco, CA: Jossey-Bass.

Wang, H-X, Karp, A., Winblad, B., \& Fratiglioni, L. (2002). Late-life engagement in social and leisure activities is associated with a decreased risk of dementia: A longitudinal study from the Kungsholmen Project. American Journal of Epidemiology, 155(12), 1081-7.

Watson, J.L., Ryan, L., Silverberg, N., Cahan, V. \& Bernard, M.A. (2014). Obstacles and opportunities in Alzheimer's clinical trial recruitment. Health Affairs (Millwood), 33(4), 574-579.

Weil, A.R. (2016). Building a culture of health. Health Affairs, 35(11), 1953-1958.

Wentz, P. (2017). Strategic communication and engaging the public. In Stock, R. \& Goldberg, B. (Eds.). Health reform policy to practice: Oregon's path to a sustainable health system: A study in innovation. (pp. 49-65). London, UK: Elsevier.

White, R. (2000). Unraveling the Tuskegee Study of Untreated Syphilis. Archives of Internal Medicine, 160, 585-598.

Wiersma, E.C. (2011). Using photovoice with people with early-stage Alzheimer's disease: A discussion of methodology. Dementia, 10(2), 203-216.

Williams, M., Scharff, D.P., Mathews, K.J., Hoffsuemmer, J.S., Jackson, P., ..., \& Edwards, D.F. (2010). Barriers and facilitators of African American participation in Alzheimer's disease biomarkers research. Alzheimer's Disease \& Associated Disorders, 24(Supp), S24-S29. 
Williams, M.M, Meisel, M.M., Williams, J. \& Morris, J.C. (2011). An interdisciplinary outreach model of African American recruitment for Alzheimer's disease research. The Gerontologist, 51(S1), S134-S141.

Williamson, O. (1975). Markets and hierarchies: Analysis and antitrust implications. New York, NY: Free Press.

Windish, D.M., Hut, S.J. \& Green, M.L. (2007). Medicine residents' understanding of the biostatistics and results in the medical literature. Journal of the American Medical Association, 298, 1010-1022.

Wood, R.Y., Giuliano, K.K., Bignell, C.U., \& Pritham, W.W. (2006). Assessing cognitive ability in research: Use of MMSE with minority populations and elderly adults with low education levels. Journal of Gerontological Nursing, 32(4), 4554.

World Health Organization (2017). Global action plan on the public health response to dementia: 2017-2025. Retrieved from: http://apps.who.int/iris/bitstream/handle/10665/259615/9789241513487eng.pdf;js essionid=9D81C306411B0AE77CDAF2EC083D9A60? sequence $=1 /$.

World Medical Association (1964). Declaration of Helsinki. Retrieved from: https://www.wma.net/wp-content/uploads/2018/07/DoH-Jun1964.pdf.

Yanniopoulou, K.G. \& Papegeorgiou, S.G. (2013). Current and future treatment for Alzheimer's disease. Therapeutic Advances in Neurological Disorders, 6(1), 1933.

Yates, L.A., Ziser, S., Spector, A. \& Orrell, M. (2016). Cognitive leisure activities and future risk of cognitive impairment and dementia: Systematic review and metaanalysis. International Psychogeriatrics, 9, 1-16.

Zweig, Y.R. \& Galvin, J.E. (2014). Lewy body dementia: The impact on patients and caregivers. Alzheimer's Research \& Therapy, 6(2), 21. 


\section{APPENDIX A: CAB WELCOME AND EXPECTATIONS}

\section{Welcome to the First Community Advisory Board Meeting!}

\section{Why does this Community Advisory Board exist?}

The purpose of this community advisory board (CAB) is to engage with me (Nicole) onPh.D. PhD research. I want to identify factors that affect a person diagnosed with memory loss's decision to participate in clinical research and develop recommendations to make it easier for them to participate.

I will do this in 4 steps. First, I will interview clinicians, researchers, and advocates of people diagnosed with memory loss. Second, I will interview people diagnosed with memory loss and their caregivers. Third, I will develop recommendations and send them to research organizations, health clinics, and advocacy groups to improve dementia clinical research participation rates based on the interview findings. Lastly, I will share the findings from this study with local community members.

\section{Why Are You Here?}

I come from a vein of science called community-engaged research, which believes that people who are impacted by something (in this case, a memory loss disorder such as Alzheimer's disease) should be at the table when researching a topic that impacts themnot just as interviewees or subjects, but as stakeholders. Further, you have lived experience of living with memory loss or have cared for someone diagnosed with memory loss, which others do not have. You are here because you are the experts!

Community-engaged research also focuses on including people whose voices have not been traditionally represented in research are not heard. For this research project, this includes people with memory loss and caregivers, and in particular older African Americans. Older African Americans are more likely to be diagnosed with memory loss and serve as caregivers, but are less likely to participate in clinical research. Furthermore, their voices are not frequently included so it's important their expertise is sought out and included in the $\mathrm{CAB}$ and from the interviewees.

Given the nature of a dissertation (where I prove that I can conduct research independently and in a timely manner), I have to take the lead in decision-making. However, collaboration and the principles of community-engaged research are incredibly important to me and influences how I plan to do research now and in the future. This means that I will be responsible for creating the agenda, facilitating the conversation and making the final decisions based on CAB expertise. 


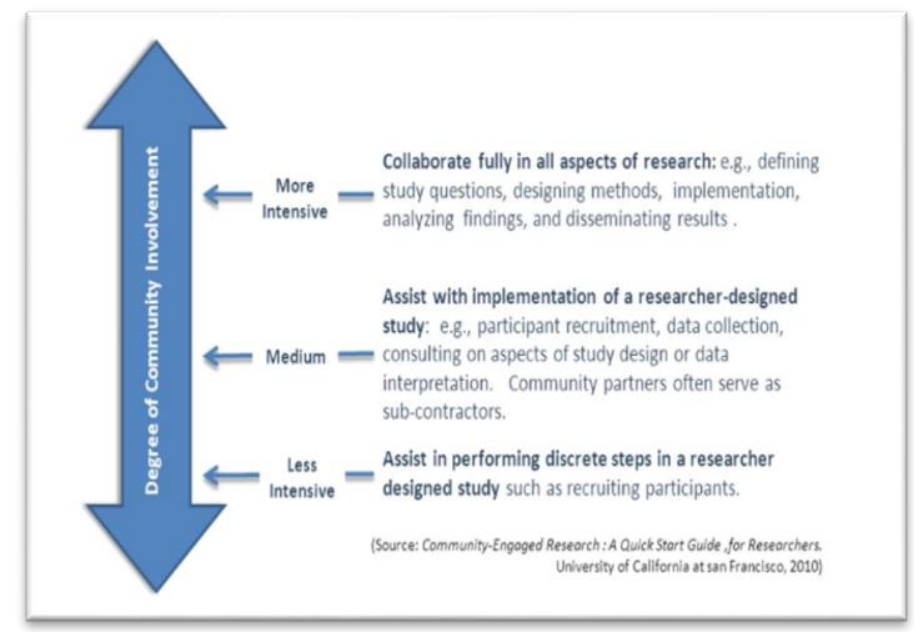

\section{What Do I Bring to the Table?}

1. Skills to facilitate the conversation among CAB members

2. Knowledge based on literature on memory loss and clinical research

3. Drafts of recruitment materials and interview questions

4. Findings from interviews

5. Ideas to share findings from this research with community members (people diagnosed with memory loss, caregivers, older African Americans, etc.)

\section{What Do You Bring to the Table?}

1. Skills to think of and discuss great ideas

2. Knowledge based on expertise in living with or caring for someone diagnosed with memory loss

3. Ideas to improve recruitment materials and interview questions

4. Identifying what's most important from the findings based on your expertise

5. Ideas to share findings from this research with community members (people diagnosed with memory loss, caregivers, older African Americans, etc.)

6. What else? 


\section{The Big Picture: Why Does This Research and the CAB Matter?}

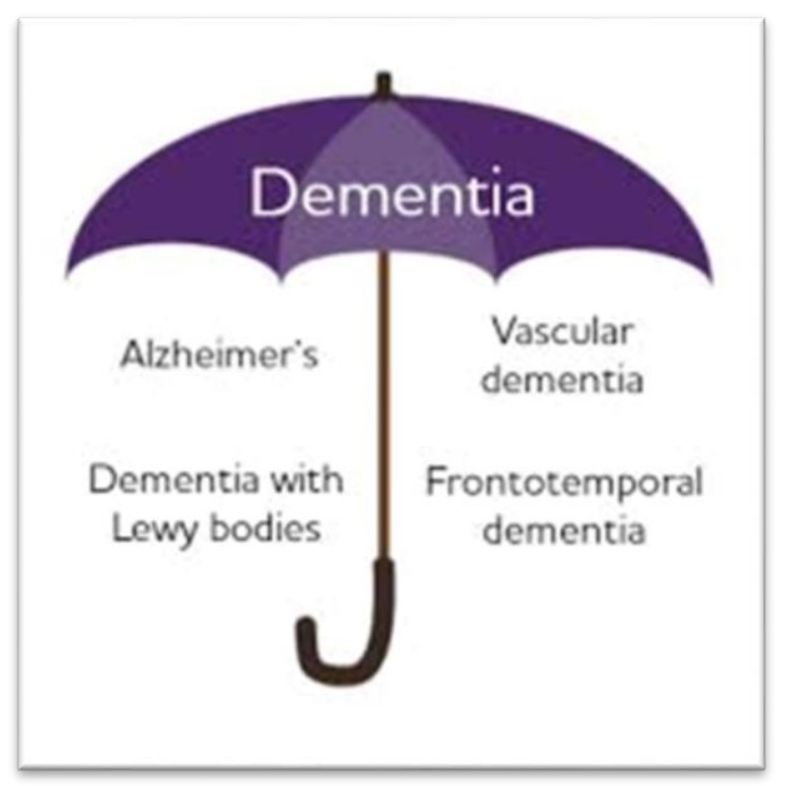

Source: Alzheimer’s Association, 2018

Memory loss disorders such as Alzheimer's disease and other dementias takes a social, financial, and medical toll on the person diagnosed, their friends and family, and their communities. There is no prevention, treatment, or cure, and number of people diagnosed and impacted by these disorders is growing fast.

- 65,000 Oregonians are diagnosed with Alzheimer's disease. This number is expected to increase $30 \%$ to 84,000 by $2025^{4}$. This doesn't include people with other forms of dementia.

Finding a preventive strategy, treatment, or cure matters to the more than 65,000 Oregonians diagnosed with memory loss, their friends and family members, and their communities. In order to find an intervention, we need people with memory loss to participate in clinical research. However, the rates of people with memory loss participating are low, so we need to find out ways to improve those rates.

In order to determine why people diagnosed with memory loss do or don't participate in research, and to recommend ways to a) be motivated to participate and b) make it easier for participate, researchers need to incorporate your voices because you are the experts on this topic.

Furthermore, including your voices in the research design process ensures that the research is designed in a way that will be most impactful to people diagnosed with

\footnotetext{
${ }^{4}$ Alzheimer's Association (2018). 2018 Alzheimer's disease Facts and Figures. Retrieved from: https://www.alz.org/media/Documents/facts-and-figures-2018-r.pdf
} 
memory loss, their friends and family members, and their local communities within the Portland metro area.

\section{Membership Responsibilities:}

1. Meeting materials are sent out at least one week prior to the meeting via email. I will include questions to prompt feedback. All members will review the material ahead of time and provide feedback at the meetings. I will send out a reminder email 48-72 hours before the meeting (or phone call, if preferred).

2. If a member is unable to attend in-person, members may join the meeting through telephone or video-chat. Members may also submit their feedback and edits prior to the meeting if they cannot attend. Members can still contribute even if they are unable to attend all 4 meetings.

3. All community members are paid $\$ 30$ grocery gift cards for attending each meeting, for up to $\$ 120$ total. Community members are defined as older African Americans, people diagnosed with memory loss, and current/former caregivers of people diagnosed with memory loss.

\section{Meeting Expectations:}

1. I will write up the meeting materials in a brief, clear manner. Exceptions may include the interview questions.

2. If a member requests it, I will meet or chat with anyone ahead of the meeting to review materials and obtain their feedback.

3. There will be 2 note-takers and time-keepers for each meeting, one for each half.

4. At the beginning of every meeting, I will review the expectations and ask if there are motions to clarify or modify them.

5. All attending members will give feedback. If changes are being made, all members need to be okay with the changes taking place. This doesn't mean they have to love the decision, but rather than they are okay with it enough for it to move forward. I will ultimately make the final decision to include each change in the final materials.

6. At the end of each discussion, the $\mathrm{CAB}$ will wait 1 minute for thought-processing or to make a final comment/suggestion before moving on to the next agenda item.

7. The CAB will take breaks as needed. Any other accommodations requested by $\mathrm{CAB}$ members will be included in the $\mathrm{CAB}$ procedures.

8. I will send out meeting notes to all members within 48 hours of the meeting. 


\section{CAB Schedule:}

Meeting dates are based first on the availability of community members, followed by Dawn Richardson, Ph.D.(PhD advisor) availability, then a Doodle poll majority vote.

\begin{tabular}{|c|c|c|}
\hline Date and time & Meeting Agenda & Notes \\
\hline $\begin{array}{l}\text { March 4, } 20192- \\
4 p m(2 \mathrm{hrs})\end{array}$ & $\begin{array}{l}\text { - Introductions and overview of } \\
\text { CAB } \\
\text { - Review Phase } 1 \text { recruitment } \\
\text { material and interview questions }\end{array}$ & \\
\hline July 2019 (1 hr) & - Interpret Phase 1 findings & \multirow{2}{*}{$\begin{array}{l}\text { These two meetings can be } \\
\text { combined if the CAB } \\
\text { prefers to do so. }\end{array}$} \\
\hline $\begin{array}{l}\text { July/August } 2019 \\
\text { (1 hr) }\end{array}$ & $\begin{array}{l}\text { - Review Phase } 2 \text { recruitment } \\
\text { material and interview questions }\end{array}$ & \\
\hline $\begin{array}{l}\text { November } 2019 \\
(2 \mathrm{hrs})\end{array}$ & $\begin{array}{l}\text { - Interpret Phase } 2 \text { findings } \\
\text { - Develop strategy to share } \\
\text { findings with community } \\
\text { members } \\
\text { - Discuss future of CAB }\end{array}$ & \\
\hline
\end{tabular}




\section{APPENDIX B: FIRST CAB MEETING AGENDA AND NOTES}

First CAB Meeting, February 4, 2019, Agenda:

\begin{tabular}{|c|c|c|}
\hline Time & Agenda Item & Objective \\
\hline $2: 00-2: 10$ & Arrival \& grab food & - Get settled in \\
\hline $2: 10-2: 20$ & Welcome & - Describe the reasons for this CAB \\
\hline $2: 20-2: 40$ & Group intro & $\begin{array}{l}\text { - Learn each other's name, expertise, } \\
\text { reason for joining CAB }\end{array}$ \\
\hline $2: 40-2: 55$ & Expectations & 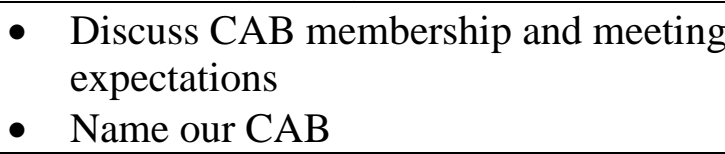 \\
\hline \multicolumn{3}{|c|}{5 minute break } \\
\hline $3-3: 24$ & $\begin{array}{l}\text { Discuss Recruitment } \\
\text { Materials }\end{array}$ & $\begin{array}{l}\text { - Enhance the recruitment list and } \\
\text { materials for Phase } 1 \text { interviewees to } \\
\text { send out }\end{array}$ \\
\hline \multicolumn{3}{|c|}{1 minute for thought processing } \\
\hline $3: 25-3: 49$ & $\begin{array}{l}\text { Discuss Interview } \\
\text { Questions }\end{array}$ & - Improve Phase 1 interview questions \\
\hline \multicolumn{3}{|c|}{1 minute for thought processing } \\
\hline $3: 50-4: 00$ & Debrief & $\begin{array}{l}\text { - Find out what worked well in this } \\
\text { meeting and communication } \\
\text { - Find out what could be improved upon }\end{array}$ \\
\hline
\end{tabular}

Note-taker 1:

Time-keeper 1:

Note-taker 2:

Time-keeper 2: 
Bouranis Community Advisory Board- First Meeting

Monday, February $4^{\text {th }}, 2019,2 \mathrm{pm}-4 \mathrm{pm}$ at New Seasons Market

In attendance: [Redacted]

Not in attendance: [Redacted]

*****************************************************************************

- Nicole introduced herself and the subject of her dissertation

- Find out why people either choose to or choose not to participate in research

- She will develop recommendations to improve research participation rates

- She will share findings from study with community members in Portland

- Nicole represents community engaged research and that's why she is bringing together people directly affected by A\&D

- She is also focused on including underrepresented groups

Attendees:

- $\quad$ Redacted]- living with dementia. Joined advisory board, because her husband is always hoping to find something new. She's hoping to learn something from it.

- [Redacted]- his wife is living with dementia. He states he's in denial about the diagnosis. Has been experiencing another family who has a dx of ALS, and in interacting with other caregiver support groups and doesn't hear a lot of hope. Would like to find ways to help so there can be hope for caregivers. Experiencing frustration and depression.

- [Redacted]- Has been involved in research about 20 years with various academic institutions. His wife got sick about four years ago and he is caring for her.

Recruits for research and wasn't sure why he was getting so many no's. Has a dx of Parkinson's disease.

- [Redacted]- Program director for Alzheimer's Association. Invited to the group as an advocate for people living with AD and other dementias.

- [Redacted]- Cared for his mother, she passed away from dementia 6 years ago. He began attending a support group in Tigard while caring for her, and now facilitates two support groups. He volunteers for the Alzheimer's association on both the care and support and fundraising side. He is currently involved in two clinical studies. He is part of research because he wants to help find a cure.

- [Redacted]- Found out about the program because they are part of an in home monitoring program. She is hoping to gather more skills as a caregiver. She expressed that it's hard for her to wrap her head around the changes with her husband, and it's frustrating that he isn't who he used to be anymore. She wants to help with research. She and her husband have explored alternative treatments for dementia, including coconut oil.

- [Redacted]- Living with Dementia. He and Jerry have been married for 50 years. Talked about how music is helpful. Phil's wife brought him to the meeting. Has not been diagnosed. 
- [Redacted]- Research assistant at the Layton center. Serves on the PreSERVE coalition with Nicole. Joined the advisory board because she is interested in Nicole's work and how to bring more people to research.

- [Redacted]- Nicole's PhD advisor, was not able to be here due to a family emergency.

- [Redacted] and [Redacted] are members of the group but weren't able to be here today due to schedules. They have provided feedback on the materials.

CAB expectations

- Nicole will send out meeting materials in advance, members should review and write down feedback for the items.

- Each member of the group will receive a $\$ 30$ gift card for each meeting attended.

- There will be note taker and time keeper for each meeting.

- Nicole asked if each group member would let her know if there is anything that would help them to focus/and/or participate in meetings to let her know privately or share with the group.

5 minute break, group to reconvene at 2:47

Action items:

- Recruitment email: It was suggested that memory loss be changed to MCI, Alzheimer's or other dementia. Feedback was given that it was not clear that the interview with the caregiver would be separate from the person with dementia interview. Nicole will work on the wording.

- Recruitment flyer: combine first two sentences about caring for or a person with MCI, dementia etc. Delete sentence with your responses to a list of factors.

- Reviewed interview list: [Redacted] suggested [Redacted], she's a PCP with house call MD. Discussed public policy director with Alz Association \{Redacted]. Harvey suggested someone with Multnomah County Health. Alz Association facilitator. Heidi to intro Nicole to [Redacted] with Summit. Harvey suggested [Redacted] to represent veterans.

- Review of interview questions: [Redacted] asked about the value of clinical research question, requested clarification. [Redacted] brought up clarification of early stage dementia for the interviews, since that is clearly outlined in the interview questions. It was suggested that ADRD be spelled out at the beginning and then can be abbreviated after, this will be changed. Nicole will change the value of clinical research to how high of a priority is clinical research. Will fix typo in form.

Debrief of meeting:

- $\quad$ [Redacted] asked how the meeting location was determined. Nicole said she looked for someplace free, with parking and that would allow her to serve food. [Redacted] suggested that earlier in the day would be better for traffic considerations. Nicole brought up the meeting schedule for the summer, we are 
scheduled to have two shorter meetings in the summer, but we could combine the two meetings into one. 


\section{APPENDIX C: SECOND CAB MEETING AGENDA AND NOTES}

Date and time: Tuesday, July $9^{\text {th }} 2019,10 \mathrm{am}-12 \mathrm{pm}$

Location: Alzheimer's Association Portland office

\begin{tabular}{|l|l|l|}
\hline Time & Agenda Item & Objective \\
\hline 10:00-10:10 & Arrival \& grab food & $\bullet$ Get settled in \\
\hline 10:10-10:25 & $\begin{array}{l}\text { Introductions and } \\
\text { Expectations }\end{array}$ & $\bullet \begin{array}{l}\text { Introductions and review CAB } \\
\text { expectations }\end{array}$ \\
\hline 10:25-11:05 & $\begin{array}{l}\text { Interpret Phase 1 } \\
\text { findings }\end{array}$ & $\bullet \begin{array}{l}\text { Identify what's most important, } \\
\text { surprising, and missing }\end{array}$ \\
\hline \multicolumn{3}{|c|}{$\mathbf{5}$ minute break } \\
\hline 11:10-11:29 & $\begin{array}{l}\text { Discuss Recruitment } \\
\text { Materials }\end{array}$ & $\begin{array}{l}\text { Enhance the recruitment list and phone } \\
\text { script for Phase 2 interviewees }\end{array}$ \\
\hline \multicolumn{3}{|c|}{ 1 minute for thought processing } \\
\hline 11:30-11:50 & $\begin{array}{l}\text { Discuss Interview } \\
\text { Questions and materials }\end{array}$ & $\begin{array}{l}\text { Improve Phase 2 interview questions } \\
\text { (includes list of factors affecting } \\
\text { decision to participate) }\end{array}$ \\
\hline & \multicolumn{2}{|c|}{ 1 minute for thought processing } \\
\hline 11:51-12:00 & Debrief & $\begin{array}{l}\text { Find out what worked well in this } \\
\text { meeting and communication } \\
\text { Find out what could be improved upon }\end{array}$ \\
\hline
\end{tabular}

Note-taker 1:

Note-taker 2:
Time-keeper 1:

Time-keeper 2: 
Bouranis Community Advisory Board - Second Meeting

Friday, Tuesday, July $9^{\text {th }} 2019,10 \mathrm{am}-12 \mathrm{pm}$ at the Alzheimer's Association

In attendance: [Redacted]

Not in attendance: [Redacted]

********************************************************************************

Nicole reviewed expectations for participation in the CAB and clarified the participatory process.

Overview of agenda provided. Introduction of the broad themes found (on posters)

Nicole sent out summary of the findings. Asked CAB to review and provide reflections

on the findings she'd emailed out and listed on posters.

$\mathrm{CAB}$ takes time to review findings, distribute post-its, share some initial thoughts:

- [Redacted]: a lot of work to do moving forward

- $\quad$ [Redacted]: can't think of anything not being captured (yet)

Nicole leads discussion and unpacking of post-it/findings review process

- Role of geriatrician as one of the most important factors- why?

$\circ$ [Redacted]: Gerontologist doesn't know history of patient

[Redacted]: Primary care physician referred to geriatrics at VA

$\circ \quad$ [Redacted]: Too often the PC has little to no geriatric training so getting to a specialist is important

- Alzheimer Association was important for engagement \& connection \& resources

$\circ \quad$ [Redacted]: Not referred to AA, but here now. Does VA work with AA?

No one there mentioned it to us.

○ [Redacted]: Was surprised at the extent. Knows it's a great resources, but

- [Redacted]: We were referred to AA, went through OHSU (10 years ago) and used the resources there

- [Redacted]: AA has been working w/physicians to educate on the importance of early diagnosis and referral, so that families will be provided with resources at diagnosis. Re: findings, it could be there is a skewed perception here, since the people sampled might be more familiar w/AA than others.

- Policies \& Funding Mechanisms (research activities dominated by sponsors)

O [Redacted]: Surprising

O [Redacted]: Surprising

○ [Redacted]: Wanted more detail

- Nicole: Industry funding was found to drive decision making. There are policies for increasing diversity in research recruitment.

- [Redacted]: Policies around research recruitment is very important, as far as why or why not people participate

- Partnerships \& Collaboration

○ [Redacted]: Wanted more detail; Finds important

- [Redacted]: Surprised that community collaboration is something they're required to make up later 
[ [Redacted]: This whole page could be greens for me

$\circ$ [Redacted]: Surprising that people w/ADRD not included- the thought process from people w/in this field is surprising

- Clinical research as a value

○ [Redacted]: Surprising. Thinks this should be such a major priority.

○ [Redacted]: Surprising.

$\circ$ [Redacted]: The doctor should be knowing more than I do

- Perceptions of Clinical Research

$\circ$ [Redacted]: Wants more detail

○ [Redacted]: Wants more detail.

$\circ \quad$ [Redacted]: Surprising. I should look at home experience. There's a study we're a part of where the questions make him angry (because he can't answer them).

- [Redacted]: I have 3 organizations asking the same stupid questions.

- Nicole: Need to reframe dementia as a public health issue; Comparison to cancer and how it's been reframed. Emphasis on resiliency.

- [Redacted]: I think it's important that people get reprogrammed. Because it's less of a thing to be ashamed of- it's something that happens. This man is almost 85 years old, and $90 \%$ of the time he's all there. People don't want to talk about it. But stop-it's ok. It should be more of a public health issue.

- Nicole: Historical relationship that has damaged the relationship between black Americans and the medical community.

- [Redacted]: Wanted sisters to know they were loved and cared for throughout the whole process; and that she was supporting them as best as possible.

\section{Recruitment Conversation}

[Redacted]: Convene a meeting with the organizations to have a discussion about this project, and see if they'd be willing to work together, with Nicole.

[Redacted]: How about senior centers? Potlucks, guest speakers- great way to connect Nicole: Would either of you be willing to connect me to your senior centers?

[Redacted]: I know people at Tigard \& Tualatin, Wilsonville too.

[Redacted]: How will you reach people "on the edge" of Alzheimers? Might need a flyer. You've also got DHS you can work with.

[Redacted]: - Memory cafes.

[Redacted]: Memory café directory. And what about Next Door? Have enough people posting on Next Door you'd reach a lot of household.

[Redacted]: Community Action Team. They have a finder on the pulse of where people are.

\section{Interview Questions Conversation}

[See Interview questions edit document] 


\section{List of Questions Conversation}

Agree/disagree? Options?

It is a long list of factors- it feels like a lot.

[Redacted]: use these, but divide in to 3 sections. Make print larger ([Redacted]: yes).

[Redacted]: It's all jammed together

[Redacted]: Some repetitiveness, and some things that are facilitators and others that are barriers.

[Redacted]: It seems like you want to know their thought process, rather than just yes/no [Redacted]: Might have implications for paycheck/benefits. A concern that some might have.

[Redacted]: Reading level should be at $6^{\text {th }}$ grade level. Condense and simplify where you can.

\section{Meeting Debrief}

[Redacted]: Post-its was a good exercise. Helps to know what you're needing. [Redacted]: We were able to get involved. If your committee understand the concept here, we do have productive meetings because we know what you're working towards. [Redacted]: This meeting felt a lot more productive. Felt like good work. My only preference: $10 \mathrm{am}$ is too early for getting here. 


\section{APPENDIX D: THIRD CAB MEETING AGENDA AND NOTES}

\section{Third CAB Meeting Agenda:}

Date and time: Friday, Jan $17^{\text {th }}$, 2020, 11:30am-1:30pm

Location: Alzheimer's Association Portland office

\begin{tabular}{|c|c|c|}
\hline Time & Agenda Item & Objective \\
\hline $11: 30-11: 40$ & Arrival \& grab food & - Get settled in \\
\hline $11: 40-11: 55$ & $\begin{array}{l}\text { Introductions and } \\
\text { Expectations }\end{array}$ & $\begin{array}{ll}\text { - Introductions and review } \mathrm{CAB} \\
\text { expectations }\end{array}$ \\
\hline $11: 55-12: 35$ & Interpret Phase 2 findings & $\begin{array}{l}\text { - Identify what's most important, } \\
\text { surprising, and missing }\end{array}$ \\
\hline \multicolumn{3}{|c|}{5 minute break } \\
\hline $12: 40-12: 55$ & $\begin{array}{l}\text { Discuss Six } \\
\text { Recommendations }\end{array}$ & $\begin{array}{l}\text { - Identify what's most important, } \\
\text { surprising, and missing }\end{array}$ \\
\hline \multicolumn{3}{|c|}{1 minute for thought processing } \\
\hline $12: 56-1: 20$ & $\begin{array}{l}\text { Discuss Dissemination } \\
\text { event }\end{array}$ & $\begin{array}{l}\text { What type of event is best way to share } \\
\text { findings with community? } \\
\text { - What else should the event include } \\
\text { (e.g. food, dancing, activity, etc.) } \\
\text { - What community resources can be } \\
\text { shared at event? } \\
\text { - Other ways to share findings }\end{array}$ \\
\hline \multicolumn{3}{|c|}{1 minute for thought processing } \\
\hline $1: 21-1: 30$ & Debrief & $\begin{array}{l}\text { - Find out what worked well in this } \\
\text { meeting and communication } \\
\text { - } \quad \text { Future collaborations }\end{array}$ \\
\hline
\end{tabular}

Note-taker 1:

Note-taker 2:
Time-keeper 1:

Time-keeper 2: 
Bouranis Community Advisory Board - Third Meeting

Friday, Jan 17, 2020 11:30-1:30 at the Alzheimer's Association

Attendees in person: [Redacted]

Attendees via phone: [Redacted]

$* * * * * * * * * * * * * * * * * * * * * * * * * * * * * * * * * * * * * * * * * * * * * * * * * * * * * * * * * * * * * * * * * * * * * * * * * * * *$

\section{Review of Findings}

\section{Theme 1: Symptom Recognition and Diagnosis}

Subtheme: Symptom recognition

- [Redacted] thought symptom recognition was important. A lot of people do not have enough knowledge to recognize symptoms. Some people may be used to people doing things for them for their whole life, and it may mask symptoms as this seems "normal".

- [Redacted] found it surprising that people pass off symptoms as normal aging. She felt she noticed symptoms in her husband earlier.

- [Redacted] found it surprising that symptoms were overlooked often.

- [Redacted] had noticed that his wife had always made lists to go to the grocery store and she always repeated things. Bonnie went to the neurologist due to forgetting students' name. Bonnie was diagnosed with younger onset Alzheimer's. After that. [Redacted] noticed her symptoms before anyone else. [Redacted] reports that she is still capable of doing everything she used to do. It's hard for her when her husband tells her that she has already said something.

- [Redacted] marked this finding as important and a bit surprising. He did report being in denial with his mom, he knew there was something wrong but didn't think it could be dementia. Availability of specialists is an issue with receiving dx.

Subtheme: Diagnosis:

- [Redacted] was surprised that doctors are not as educated as they should be on diagnosis.

- [Redacted] stated that there have been a lot of efforts to educate physicians, and this is improving some. Shared that GP's are tasked with so much. We don't have great medical treatments for dementia, and that can be difficult for docs and this can be a reason for hesitancy with dx. Primary care docs may have known patients for a long period of time, and may also be in denial. Competing interests i.e. cancer and heart.

\section{Theme 2: Factors Affecting Ability to Participate}

Subtheme: Knowledge of study opportunities

- [Redacted] wanted more info on how to find study opportunities.

- [Redacted] shared that when [Redacted] was referred to go to a neurologist in Hillsboro. [Redacted] was shocked that her meeting was at 9am and was supposed to be there $45 \mathrm{~min}$. They kept her there until $3 \mathrm{pm}$, and she was a "mess". He went to get a second opinion, and she was diagnosed with MCI. Dr. called at $8: 15 \mathrm{pm}$, and said that [Redacted] had Alzheimer's. He did not offer 
support. Wants to be given lifestyle interventions instead of just drugs.

Caregivers are dying faster than the patients.

Subtheme: Ineligibility/forced withdrawal from study

- [Redacted] was surprised about people not being able to be in studies, or studies being cancelled. [Redacted] agreed.

Subtheme: Time/Distance

Subtheme: Caregiver Burden

- [Redacted] asked about the Caregiver burden, and participant burden. [Redacted] reported as a clinically normal study participant that he does feel the participant burden. He feels strongly that it is important to participate.

Subtheme: Using the internet

Theme 3: Motivation to Participate in Research

Subtheme: Motivation to help others

- $\quad$ Redacted] found this important

Subtheme: Living life to the fullest

- $\quad$ Redacted] would like to see something positive come out of research.

- [Redacted] noted this was very important. It resonated with her, as she has worked with a lot of study participants.

Subtheme: Taking study drugs

- [Redacted] said it was important because people are being asked to take drugs that have not been fully tested and do not understand side effects.

- $\quad$ [Redacted] found it surprising and wondered about distrust of medical community.

- $\quad$ [Redacted] expressed distrust of pharmaceutical industry.

- [Redacted] talked about having a range of trials available lowest to highest risk.

- [Redacted] shared that many commercials are pharmaceutical in nature, and side effects are worse than the disease. The first thing the neurologist did was to prescribe medications. Would rather have [Redacted] be happy then be drugged.

- [Redacted] asked if dementia is genetic. Nicole and [Redacted] shared that it can be. [Redacted] shared that there are also environmental and lifestyle concerns as well.

- [Redacted] reported that African Americans have historicaly been taken advantage of in studies.

Subtheme: Caregiver Support

- [Redacted] said this is more disappointing than surprising.

Subtheme: Advisory board participation

- $\quad$ Redacted] found this interesting 


\section{Six Recommendations}

1. Identify and promote local champions for ADRD clinical research participation

2. Promote policies and processes that incentivze collaboration between organizations

3. Recognize caregivers as full research participants

4. Include people with ADRD and caregivers in the research design process

5. Offer alternative options to reduce participation burden

- [Redacted] noted it would be helpful if research staff can go to the local clinics, in a setting where they would feel more comfortable

6. Evaluate and improve relationships between healthcare/research staff and patients/participants

- [Redacted] brought up pharmacist education

\section{Sharing These Findings with the Community}

- Set up a booth at already-established community events (they don't need to be specific to dementia) to share information about dementia research that people with dementia and caregivers that may already be attending those events

- [Redacted] noted Talk and Tastes

- Presentations at McGinty conference

- People probably would not come out for a presentation that is just on this topic.

- Future articles, presentations, writings- some CAB members would be interested in reviewing 


\section{APPENDIX E: RECRUITMENT EMAIL}

Hi there.

My name is Nicole Bouranis. I'm a doctoral student at the Oregon Heath \& Science University-Portland State University School of Public Health. I'm interested in clinical research for memory loss disorders. Specifically, I want to know all of the reasons why people diagnosed with MCI, Alzheimer's disease, or another related dementia do or don't participate in clinical research. For my dissertation, I'm planning to interview people who are experts on living with memory loss or caring for a family member or friend diagnosed with memory loss. This email is an invitation to you to participate in the study.

Who do I want to interview? People who have been diagnosed with memory loss and their primary caregivers (friend or family member).

What would we talk about? We would talk about the following topics:

- When signs or symptoms of memory loss were first noticed, and the diagnosis experience.

- Why people diagnosed with memory loss are or aren't motivated to participate in clinical research.

- What makes it easier or more difficult to participate in clinical research.

How long will this interview last and where would it take place? You and your friend/family member would be interviewed separately. Each interview would last between 30-60 minutes and take place in the home of the person with memory loss (or another preferred location).

Will I get paid for this? Yes! You and your friend/family member will each receive a $\$ 20$ gift card to a preferred retailer.

This sounds great! How can I get involved? If you are interested in learning more about the study or being interviewed, please contact me at Bour4@pdx.edu or XXX$\mathrm{XXX}-\mathrm{XXXX}$. If you are not interested, please also let me know so that I will not continue to contact you.

Thank you in advance. I look forward to hearing from you, Nicole Bouranis 


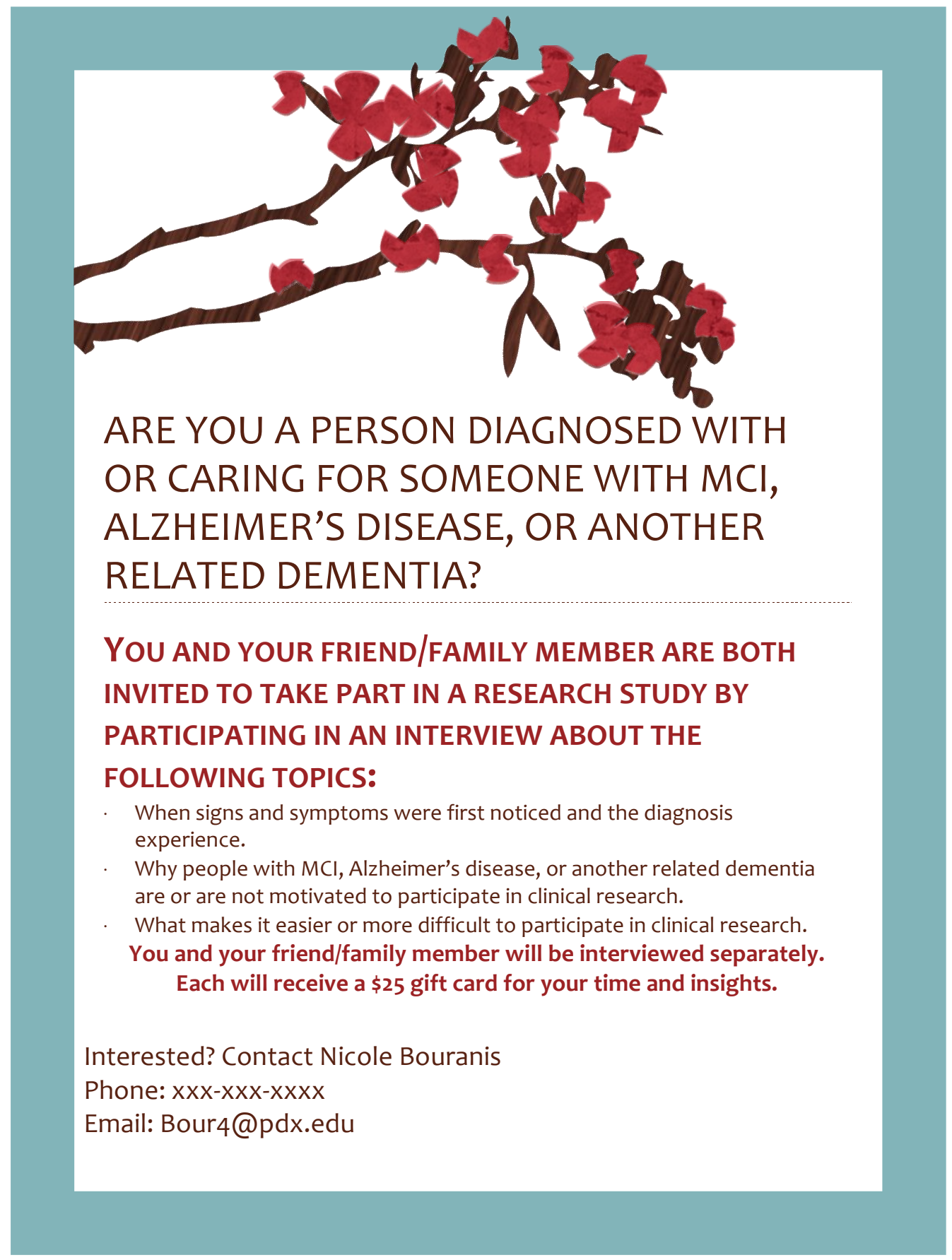




\section{APPENDIX G: SCREENING PHONE SCRIPT}

Hello, is this $\mathrm{xxxx}$ or $\mathrm{xxx}$ ?

If wrong number, thank them and hang up.

If yes, Hi xxxx, this is Nicole Bouranis. I'm calling because you emailed [me]/[key informant]/ [other recruiter] about a study I'm working on, and said you might be interested in participating. Is that correct?

If no, thank them and hang up.

If yes, Is now a good time to talk?

If no, ask when is a good time to call back, and schedule time to call back. Provide them contact information.

If yes, Great. So to give you background on the study, I am a doctoral student at the Oregon Health and Science University - Portland State University School of Public Health. My research looks at the different types of factors that affect a person with memory loss's ability and motivation to participate in clinical research. We hope to better understand what helps or gets in the way of a person's decision to enroll in a clinical research study by interviewing both people who have been diagnosed with memory loss and their friend or family member who acts as their primary caregiver. The interview will ask some questions about memory loss and about clinical research.

Through these interviews, we hope to improve access and motivation to participate, increase enrollment, and find a cure, treatment, or prevention for memory loss diseases such as mild cognitive impairment, Alzheimer's disease and related dementias. Interviews should last about 90 minutes total and will be audio-recorded. The recording of the interview will be erased once it has been written up, and your name will not appear in any written documentation or any other places where the study results are shared. To thank you for your time, both you and your friend/family member will each receive a $\$ 20$ gift card to your preferred retailer.

Are you still interested?

If no, thank them and hang up.

If yes, Great. I'd like to continue by asking you some eligibility questions. This will just take a few minutes.

First, have you or your friend/family member been given a diagnosis by a doctor or other clinician of mild cognitive impairment (MCI), Alzheimer's disease, or another related 
dementia, such as Lewy-body dementia, frontotemporal dementia (FTD), vascular dementia, or mixed dementia?

If the person responds with any form of ADRD: Are you or your friend/family member considered to be in the early or moderate stages as per your/their last provider visit within the last six months?

Second, do you and your friend/family member speak fluent English?

Third, are you and your friend/family member able to engage in a conversation about your experiences with being diagnosed with [diagnosis], living with [diagnosis], and your perceived values about clinical research?

Finally, will you and your friend/family member both be able to individually sign a consent form?

If no to any of these questions: Unfortunately, it looks like you do not meet the eligibility criteria for this study. Thanks for your interest. If you know anyone who would meet the criteria and think they might be interested in participating, please give them my information and tell them to reach out to me.

If yes, Wonderful, you and your friend/family member meet all the criteria for participating in the interview. One last question: have you/your friend/family member participated in clinical research related to memory loss previously?

Set up a time and location for the interview.

I'm going to send you and your friend/family member the consent forms ahead of the interview for your review. Do you prefer email or regular mail? What are your addresses/email addresses?

Great, I'll see you on DATE at LOCATION. I'll call you the day before to remind you. If you need to postpone or cancel, please call me at XXX-XXX-XXXX or email me at Bour4@pdx.edu. I look forward to seeing you and xxxx. 


\section{APPENDIX H: KEY INFORMANT CONSENT FORM}

\section{Portland State University \\ Consent to Participate in Research \\ Multi-level factors affecting clinical research enrollment among people with Alzheimer's disease or related dementias (ADRD)}

Study By: Nicole Bouranis, MA, Doctoral Student, Bour4@pdx.edu

Principal Investigator: Dr. Sherril Gelmon, Dissertation Committee Chair, gelmons@pdx.edu

\section{What is this form for?}

We invite you to participate in a research study being conducted by Nicole Bouranis, a doctoral student at the School of Public Health at Oregon Health \& Science University and Portland State University. This form will explain the research study and the possible risks and benefits to you if you decide to participate. If you have any questions, please ask Nicole Bouranis.

\section{Why am I being asked to participate?}

You are a clinician who treats people with ADRD, an investigator or research assistant for ADRD clinical research, or an advocate for people with ADRD.

\section{What is this study about?}

This study looks at the different types of factors that affect a person with MCI, Alzheimer's disease or a related dementia's ability and motivation to participate in clinical research. We hope to better understand what helps or gets in the way of a person's decision to enroll in a clinical research study. By doing so, we hope to improve access and motivation to participate, increase enrollment, and find a cure, treatment, or prevention for Alzheimer's disease and related dementias.

\section{What will happen if I decide to participate?}

If you agree to be in the study, you will participate in an interview with Nicole Bouranis. This interview will take place on [DATE]. During this interview you will talk about:

- Your and your organization's engagement with people with ADRD.

- Policies and funding mechanisms that affect your engagement with people with ADRD.

- Partnerships that engage with or relate to people with ADRD.

- How valued clinical research for ARDD is within your organization. 
- Your perceptions of what makes it easier or more difficult to recruit people with ADRD into clinical research.

The interview will be audio-taped.

You do not have to answer any questions that you do not want to. You can end the interview and your participation in this study at any time.

\section{How long will this study take?}

This interview will last about one hour.

\section{What are the risks of being in this study?}

It is very unlikely that participating in this study could cause you harm as the questions discussed relate to organizational and policy elements, not personal experiences. There is also a risk of loss of privacy if the researcher fails to follow the strict rules about confidential information, but there are many safeguards in place to prevent this. For more information about risks and discomforts, please ask Nicole Bouranis.

\section{What are the benefits to being in this study?}

Participating in the interview may or may not be helpful to you. However, your answers may help make it easier to improve access for people with MCI or ADRD to participate in clinical research in the future.

\section{How will my information and data be kept private or confidential?}

When the project is complete, there will be a report of the results. Your name or information that might identify you will not be in the report. Everyone involved in the study will be trained to keep your information private. The recording of the interview will be erased once it has been written up, and your name will not appear in any written documentation or any other places where the study results are shared. Information from this study will be kept on a password-protected computer.

\section{Will I be paid for taking part in this study?}

No.

\section{Can I stop being in the study once I begin?}

You do not have to be in this research study. You have the right to change your mind about being in the study at any time. Your participation in this study will help researchers find ways to improve participation in clinical research for MCI and ADRD.

\section{Who can I contact with questions or complaints about this study?}


If you have any questions, concerns, or complaints at any time about the research study, please contact Nicole Bouranis at XXX-XXX-XXXX or Bour4@pdx.edu.

\section{Who can I contact with questions about my rights as a research participant?}

If you have questions regarding your rights as a research participant, you may contact the Portland State University (PSU) Office for Research Integrity at (503) 725-2227 or 1 (877) 480-4400 or email hsrrc@pdx.edu. The ORI is the office that supports the PSU Institutional Review Board (IRB).

\section{CONSENT}

You are making a decision about whether to participate in this study. By signing below, you indicate that you have read the information in this form, have had an opportunity to ask any questions and have them answered, and are agreeing to participate in the study. A copy of this consent form will be given to you, if you request it.

Your signature below indicates that you have read the information provided (or the information was read to you). By signing this consent form, you are not waiving any of your legal rights as a research participant.

Name of Adult Subject (print) Signature of Adult Subject $\quad$ Date

\section{INVESTIGATOR SIGNATURE}

This research study has been explained to the participant and all of his/her questions have been answered. The participant understands the information described in this consent form and freely consents to participate.

Name of Investigator/ Research Team Member (type or print)

(Signature of Investigator/ Research Team Member) Date 


\section{APPENDIX I: CAREGIVER CONSENT FORM}

\section{Portland State University \\ Consent to Participate in Research \\ Multi-level factors affecting clinical research enrollment among people with Alzheimer's disease or related dementias (ADRD)}

Study By: Nicole Bouranis, MA, Doctoral Student, Bour4@pdx.edu

Principal Investigator: Dr. Sherril Gelmon, Dissertation Committee Chair, gelmons@pdx.edu

\section{What is this form for?}

We invite you to participate in a research study being conducted by Nicole Bouranis, a doctoral student at the School of Public Health at Oregon Health \& Science University and Portland State University. This form will explain the research study and the possible risks and benefits to you if you decide to participate. If you have any questions, please ask Nicole Bouranis.

\section{Why am I being asked to participate?}

You are a person who is a primary caregiver of someone who has been diagnosed with MCI, Alzheimer's disease, or a related dementia. ADRD research requires the subject to have a study partner to attend appointments and make sure the person with ADRD adheres to study protocols. This means that your ability and motivation to participate in a clinical research is just as necessary as the person with MCI or ADRD.

\section{What is this study about?}

This study looks at the different types of factors that affect the ability and motivation to participate in clinical research pf a person with MCI, Alzheimer's disease or a related dementia. We hope to better understand what helps or gets in the way of a person's decision to enroll in a clinical research study. By doing so, we hope to improve access and motivation to participate, increase enrollment, and find a cure, treatment, or prevention for Alzheimer's disease and related dementias.

\section{What will happen if I decide to participate?}

If you agree to be in the study, you will participate in an interview with Nicole Bouranis. Your friend or family member will also be interviewed. If they wish, you may sit in on their interview, but it is important that all questions in that interview are answered by the person with MCI/ADRD. This interview will take place on [DATE]. During this interview you will talk about: 
- When you noticed signs or symptoms of MCI/ADRD and when your friend or family member received an ADRD diagnosis.

- Why you are or aren't motivated to participate in clinical research.

- What makes it easier or more difficult to participate in clinical research.

- Your responses to a list of factors that make it easier or more difficult to participate in clinical research that was created by providers, researchers , and advocates.

The interview will be audio-taped.

You do not have to answer any questions that you don't want to. You can end the interview and your participation in this study at any time.

\section{How long will this study take?}

The two interviews should take no more than 90 minutes, with your interview lasting about a half hour, followed by your friend or family member's interview, which will last up to one hour.

\section{What are the risks of being in this study?}

It is very unlikely that participating in this study could cause you harm. However, it is possible that some questions or comments may bring up memories of difficult experiences with the health care system or a research organization. There is also a risk of loss of privacy if a researcher failed to follow the strict rules about confidential information, but there are safeguards in place to avoid this. For more information about risks and discomforts, please ask Nicole Bouranis.

\section{What are the benefits to being in this study?}

Participating in the interview may or may not be helpful to you. However, your answers may help make it easier to improve access for people with MCI or ADRD to participate in clinical research in the future.

\section{How will my information and data be kept private or confidential?}

When the project is complete, there will be a report of the results. Your name or information that might identify you will not be in the report. Everyone involved in the study will be trained to keep your information private. The recording of the interview will be erased once it has been written up, and your name will not appear in any written documentation or any other places where the study results are shared. Information from this study will be kept on a password-protected computer.

\section{Will I be paid for taking part in this study?}

Both you and your friend or family member will receive a $\$ 25$ gift card to your preferred grocery retailer at the end of the interview.

\section{Can I stop being in the study once I begin?}


You do not have to be in this research study. You have the right to change your mind about being in the study at any time. If you decide not to participate, you will still receive the $\$ 25$ grocery gift card. Your participation in this study will help researchers find ways to improve participation in clinical research for MCI and ADRD. 


\section{Who can I contact with questions or complaints about this study?}

If you have any questions, concerns, or complaints at any time about the research study, please contact Nicole Bouranis at XXX-XXX-XXXX or Bour4@pdx.edu.

\section{Who can I contact with questions about my rights as a research participant?}

If you have questions regarding your rights as a research participant, you may contact the Portland State University (PSU) Office for Research Integrity at (503) 725-2227 or 1 (877) 480-4400 or email hsrrc@pdx.edu. The ORI is the office that supports the PSU Institutional Review Board (IRB).

\section{CONSENT}

You are making a decision about whether to participate in this study. By signing below, you indicate that you have read the information in this form, have had an opportunity to ask any questions and have them answered, and are agreeing to participate in the study. A copy of this consent form will be given to you, if you request it.

Your signature below indicates that you have read the information provided (or the information was read to you). By signing this consent form, you are not waiving any of your legal rights as a research participant.

Name of Adult Subject (print) Signature of Adult Subject $\quad$ Date

\section{INVESTIGATOR SIGNATURE}

This research study has been explained to the participant and all of his/her questions have been answered. The participant understands the information described in this consent form and freely consents to participate.

Name of Investigator/ Research Team Member (type or print)

(Signature of Investigator/ Research Team Member) Date 


\section{APPENDIX J: PERSON WITH ADRD CONSENT FORM}

\section{Portland State University \\ Consent to Participate in Research \\ Multi-level factors affecting clinical research enrollment among people with Alzheimer's disease or related dementias (ADRD)}

Study By: Nicole Bouranis, MA, Doctoral Student, Bour4@pdx.edu

Principal Investigator: Dr. Sherril Gelmon, Dissertation Committee Chair, gelmons@pdx.edu

\section{What is this form for?}

We invite you to participate in a research study being conducted by Nicole Bouranis, a doctoral student at the School of Public Health at Oregon Health \& Science University and Portland State University. This form will explain the research study and the possible risks and benefits to you if you decide to participate. If you have any questions, please ask Nicole Bouranis.

\section{Why am I being asked to participate?}

You are a person who has been diagnosed with MCI, Alzheimer's disease, or a related dementia.

\section{What is this study about?}

This study looks at the different types of factors that affect the ability and motivation of a person with MCI, Alzheimer's disease or a related dementia to participate in clinical research. We hope to better understand what helps or gets in the way of a person's decision to enroll in a clinical research study. By doing so, we hope to improve access and motivation to participate, increase enrollment, and find a cure, treatment, or prevention for Alzheimer's disease and related dementias.

\section{What will happen if I decide to participate?}

If you agree to be in the study, you will participate in an interview with Nicole Bouranis. Your primary caregiver will also be interviewed. They are welcome to sit in on your interview if you wish, but it is important that you are the one answering the questions. This interview will take place on [DATE]. During this interview you will talk about:

- When you noticed signs or symptoms of MCI/ADRD and your ADRD diagnosis.

- Why you are or aren't motivated to participate in clinical research.

- What makes it easier or more difficult to participate in clinical research. 
- Your thoughts on a list of factors that make it easier or more difficult to participate in clinical research that was created by providers, researchers, and advocates.

The interview will be audio-taped.

You do not have to answer any questions that you don't want to. You can end the interview and your participation in the study at any time.

\section{How long will this interview take?}

This interview will take a total of up to 90 minutes. Your primary caregiver will be interviewed for up to a half hour. After the interview with your primary caregiver is finished, our interview will begin and will last for up to one hour. During your interview, you will respond to a list of factors that make it easier or more difficult to participate in clinical research. We will discuss your responses to the list during the interview

\section{What are the risks of being in this study?}

It is very unlikely that participating in this study could cause you harm. However, it is possible that some questions or comments may bring up memories of difficult times within the health care system or a research organization. There is also a risk of loss of privacy if a researcher failed to follow the strict rules about confidential information, but there are many safeguards in place to avoid this. For more information about risks and discomforts, please ask Nicole Bouranis.

\section{What are the benefits to being in this study?}

Participating in the interview may or may not be helpful to you. However, your answers may help make it easier to improve access for people with MCI or ADRD to participate in clinical research in the future.

\section{How will my information and data be kept private or confidential?}

When the project is complete, there will be a report of the results. Your name or information that might identify you will not be in the report. Everyone involved in the study will be trained to keep your information private. The recording of the interview will be erased once it has been written up, and your name will not appear in any written documentation or any other places where the study results are shared. Information from this study will be kept on a password-protected computer.

\section{Will I be paid for taking part in this study?}

You and your primary caregiver will each receive a $\$ 25$ gift card to your preferred retailer at the end of the interview.

\section{Can I stop being in the study once I begin?}


You do not have to be in this research study. You have the right to change your mind about being in the study at any time. If you decide not to participate, you will still receive the $\$ 25$ grocery gift card. Your participation in this study will help researchers find ways to improve participation in clinical research for ADRD. 


\section{Who can I contact with questions or complaints about this study?}

If you have any questions, concerns, or complaints at any time about the research study, please contact Nicole Bouranis at XXX-XXX-XXXX or Bour4@pdx.edu.

\section{Who can I contact with questions about my rights as a research participant?}

If you have questions regarding your rights as a research participant, you may contact the Portland State University (PSU) Office for Research Integrity at (503) 725-2227 or 1 (877) 480-4400 or email hsrrc@pdx.edu. The ORI is the office that supports the PSU Institutional Review Board (IRB).

\section{CONSENT}

You are making a decision about whether to participate in this study. By signing below, you indicate that you have read the information in this form, have had an opportunity to ask any questions and have them answered, and are agreeing to participate in the study. A copy of this consent form will be given to you, if you request it.

Your signature below indicates that you have read the information provided (or the information was read to you). By signing this consent form, you are not waiving any of your legal rights as a research participant.

Name of Adult Subject (print) Signature of Adult Subject $\quad$ Date

\section{INVESTIGATOR SIGNATURE}

This research study has been explained to the participant and all of his/her questions have been answered. The participant understands the information described in this consent form and freely consents to participate.

Name of Investigator/ Research Team Member (type or print)

(Signature of Investigator/ Research Team Member) Date 


\title{
APPENDIX K: KEY INFORMANT INTERVIEW PROTOCOL
}

\author{
Bouranis Dissertation \\ Study ID: \\ Subject Contact Information:
}

This study looks at the different types of factors that affect a person with MCI, Alzheimer's disease or a related dementia's, which I will refer to as ADRD throughout this interview, ability and motivation to participate in clinical research. We hope to better understand what helps or gets in the way of a person's decision to enroll in a dementia clinical research study. You are a clinician who treats people with ADRD, an investigator or research assistant for ADRD clinical research, or an advocate for people with ADRD.

This interview will last approximately one hour. During this interview, we'll discuss you and your organization's engagement with people with ADRD, policies and funding mechanisms that affect your engagement with people with ADRD, partnerships that engage with or relate to people with ADRD, how valued clinical research for ADRD is within your organization, and your perceptions of what makes it easier or more difficult to recruit people with ADRD into clinical research.

You do not have to answer any questions that you don't want to. You can end the interview and your participation in this study at any time. This interview will be audiotaped. The recording of the interview will be erased once it has been written up, and your name will not appear in any written documentation or any other places where the study results are shared.

Do you have any questions before we get started?

Okay, let's get started.

1. Describe this organization and your role within it.

a. Prompt: How does your organization engage people with ADRD?

2. What policies or funding mechanisms enhance your work?

3. What policies or funding mechanisms have made it easier or more difficult to engage with people with ADRD and/or increase knowledge of research opportunities?

4. What kinds of partnerships are you involved in that relate to people with ADRD? Please describe these briefly.

5. How is this/are these partnership(s) funded? How are stakeholders involved?

6. Describe your process for informing [recruiting, and enrolling (if organization is a research organization)] people with ADRD and their caregivers of research opportunities.

a. Prompt: Has this always been the process? How has that process changed since it was implemented? 
7. How high of a priority is clinical research at this organization?

8. What are some factors that you think may facilitate recruitment?

a. Prompt: examples may include transportation, awareness of opportunities, time, etc.

9. What about factors that impede recruitment?

a. Prompt: examples may include transportation, awareness of opportunities, time, etc.

10. What are your recommendations for increasing ADRD clinical research participation?

Thanks so much for your input; this concludes this interview. As you know, my next phase of interviews will be with people diagnosed with MCI or ADRD and their caregivers. Would you be willing to help me recruit for these interviews by posting this flyer and also sending my recruitment letter to your email list, or specific people that would eligible for my interviews? Individuals diagnosed with memory loss need to be diagnosed with or in the early stages of MCI or ADRD. They also need to be able to speak about their diagnosis experience, living with their diagnosis, and their perceptions about clinical research. Lastly, they need to be able to sign a consent form. Both dyad members also need to speak fluent English.

Remember, you can follow up with me at XXX-XXX-XXXX or Bour4@pdx.edu. When my research is finished, I will contact you again to offer you a summary of my major findings. Thanks again! 


\section{APPENDIX L: CAREGIVER INTERVIEW PROTOCOL}

\section{Bouranis Dissertation}

Study ID:

Participant contact information:

Thank you for participating in my dissertation research. As noted in the consent form, this research looks at the different types of factors that affect a person with diagnosed memory loss's ability and motivation to participate in clinical research. You are a primary caregiver of someone who has been diagnosed with memory loss. ADRD research requires that a person living with MCI, Alzheimer's disease, or a related dementia who participates as a research participant must have a study partner to attend appointments with them and make sure they adhere to study protocols. This means that your ability and motivation to participate in a clinical research study is just as necessary as the person living with MCI or ADRD.

This interview with you will take up to an hour. During the interview, we'll talk about when <person living with ADRD/MCI> was diagnosed, motivations to participate in dementia clinical research and factors that make it easier or difficult to participate in clinical research. After our interview is over, I'll interview <person living with dementia $>$ for up to an hour. You are welcome to sit in on that interview, but it is important that you let <person living with dementia> answer the questions. You can provide me with any corrections of facts or clarifications after that interview (by email or telephone).

You do not have to answer any questions that you don't want to. You can end the interview and your participation in this study at any time and you and <person living with ADRD/MCI> will still each receive the $\$ 25$ grocery gift card to [PREFERRED RETAILER]. This interview will be audio-recorded, and I'll also jot down notes on my clipboard. The recording of the interview will be erased once it has been written up, and your name will not appear in any written documentation or any other places where the study results are shared.

Do you have any questions before we get started?

Okay, let's get started.

1. When did you first notice signs of $\mathrm{MCI} /$ dementia in <person living with $\mathrm{ADRD} / \mathrm{MCI}>$ ?

2. When did they receive a diagnosis?

3. Please describe in your own words what "clinical research" is. 
a. Prompt for those who don't have an answer: Would you describe it as) any medical research involving people; $b$ ) research that involves drugs or medical procedures; or c) research that tests if one drug, device, or medical procedure works better than another one?

b. Have you ever heard of clinical research opportunities? What about clinical research for dementia?

c. What do you think about clinical research?

4. In our previous conversation, you mentioned that you and <person living with dementia $>$ have/have not participated in dementia clinical research. What made you decide to participate/not participate?

a. Prompt FOR THOSE WHO HAVE PARTICIPATED: what was your experience like? Would you participate again?

b. Prompt for all: did you attempt to join other studies but were considered ineligible? What happened?

5. Are there any barriers that made or make it more difficult for you to participate?

a. Prompt: What were they?

6. Was there anything that made or would make it easier for you to participate?

a. Prompt: what would it be?

7. What makes you not want to participate?

8. If you could tell research organizations anything to improve research participation, what would it be?

9. I'm going to show you a list of factors that make it easier or more difficult to participate in clinical research for dementia. This list was created by local providers, researchers, and advocates. Please tell me if you agree or disagree with any of these factors and why.

Thanks so much for your input. That concludes this interview, and we'll get $<$ person living with dementia> to begin their interview. Remember, you can follow up with me at XXX-XXX-XXXX or Bour4@pdx.edu. When my research is finished, I will contact you again to offer you a summary of my major findings. Thanks again! 


\section{APPENDIX M: PERSON WITH ADRD INTERVIEW PROTOCOL}

\section{Bouranis Dissertation}

Study ID:

Subject contact information:

Thank you for participating in my dissertation research. As noted in the consent form, this research looks at the different types of factors that affect a person diagnosed with memory loss's ability and motivation to participate in clinical research.

This interview will take about an hour. During the interview, we'll talk about when you were diagnosed, motivations to participate in dementia clinical research and factors that make it easier or difficult to participate in clinical research. We'll also discuss your answers to the form on factors that make it easier or difficult to participate in research.

You may choose to have <caregiver> with us for this interview, or choose to have them not here. If you choose to have them here, it's important that you're answering the questions, not <caregiver>. The caregiver already answered these questions in their interview, and we want to learn about this from your perspective.

You do not have to answer any questions that you don't want to. You can end the interview and your participation in this study at any time and you and <caregiver> will still each receive the $\$ 20$ gift card to [PREFERRED RETAILER]. This interview will be audio-recorded, and I'll also jot down notes on my clipboard. The recording of the interview will be erased once it has been written up, and your name will not appear in any written documentation or any other places where the study results are shared.

Do you have any questions before we get started?

Okay, let's get started.

1. When did you first notice signs of MCI/ADRD?

2. When did you receive a diagnosis?

3. Please describe in your own words what "clinical research" is.

4. Have you ever been aware of clinical research opportunities? How so?

a. What about dementia research opportunities?

5. What do you think about clinical research?

6. In our previous conversation, you have mentioned that you have/have not participated in dementia clinical research. What made you decide to participate/not participate?

a. Prompt FOR THOSE WHO HAVE PARTICIPATED: what was your experience like? Would you participate again?

7. Are there any barriers that made or make it more difficult for you to participate?

a. Prompt: What were they? 
8. Was there anything that made or would make it easier for you to participate?

a. Prompt: what would it be?

9. What makes you not want to participate?

10. Let's review this form you filled out. This list was created by local providers, researchers, and advocates, about factors that make it easier or more difficult to participate in clinical research for dementia.

a. Prompt for each factor: For <factor 1, 2, etc.>, you said you $<$ agree/disagree, and ...>. Why is that? Is there anything else you'd like to add?

11. If you could tell research organizations anything to improve dementia research participation, what would it be?

Okay, that concludes our interview. Thanks so much for your input. Remember, you can follow-up with me at XXX-XXX-XXXX or Bour4@pdx.edu. When my research is finished, I will contact you again to offer you a summary of my major findings. Thanks again! 


\section{APPENDIX N: LIST OF FACTORS}

Bouranis Dissertation

Date:

Study participant ID (for researcher use only, please don't fill out):

Directions: Please indicate your level of agreement with each of the following factors regarding how they affect one's decision to join dementia clinical research studies. Please circle your answer for each statement. If you have additional comments, please write them in the spaces provided on each page.

\section{Factors that Affect the Ability to Join Research}

1. Learning about research opportunities from one's provider.

2. Learning about research opportunities from a presentation or from a trusted community leader, organization, or group (e.g. senior or cultural center, support group, clergy).

3. Learning about research opportunities from flyers, newspapers, radio, tv, or social media.

4. If one's caregiver is interested and able to participate.

5. The study staff is willing to be flexible for the caregiver's schedule.

6. Being told about other study opportunities if not eligible for an initial study.

7. Options that make it easier to participate (e.g. ride to the study site, no site visits).

8. Not receiving a timely and accurate dementia diagnosis from one's healthcare provider.

Agree Disagree Don't

Know

Agree Disagree Don't

Know

Agree Disagree Don't

Know

Agree Disagree Don't

Know

Agree Disagree Don't

Know

Agree Disagree Don't

Know

Agree Disagree Don't

Know

Agree Disagree Don't

Know

Agree Disagree Don't

Know

Agree Disagree Don't

Know

Agree Disagree Don't

Know research (e.g. age, memory scores, history of disease, medications, distance from study site).

12. Finding out the study won't start for a long time after initially hearing about it.

Agree Disagree

Don't

Know

Please write any additional comments here. 


\section{Factors that Affect Motivation to Join Research}

13. The hope that research will help the person with dementia, their family, or society.

14. Being able to contribute to science.

15. Whether the study builds trust and benefits one's community.

16. The fear of being identified as a person with dementia.

$\begin{array}{lll}\text { Agree } & \text { Disagree } & \begin{array}{l}\text { Don't } \\ \text { Know }\end{array} \\ \text { Agree } & \text { Disagree } & \begin{array}{l}\text { Don't } \\ \text { Know }\end{array} \\ \text { Agree } & \text { Disagree } & \begin{array}{l}\text { Don't } \\ \text { Know }\end{array} \\ \text { Agree } & \text { Disagree } & \begin{array}{l}\text { Don't } \\ \text { Know }\end{array}\end{array}$

Agree Disagree Don't

Know

Agree Disagree Don't

Know education, support groups).

19. Being able to try a new medication.

20. Receiving regular updates about the study.

21. Public awareness/opinions about dementia and dementia clinical research.

22. Ability to socialize within research.

23. The fear that nothing can be done even if one does participate.

Please write any additional comments here.

24. Being paid an adequate amount to participate in the study (and without affecting benefits).

25. Study recruitment materials (e.g. flyers) that feature people who look like the person with dementia.

26. Study staff that look like the person with dementia and have a shared socio-historical background or other shared connection.

27. Being a part of an advisory board that collaborates on research.

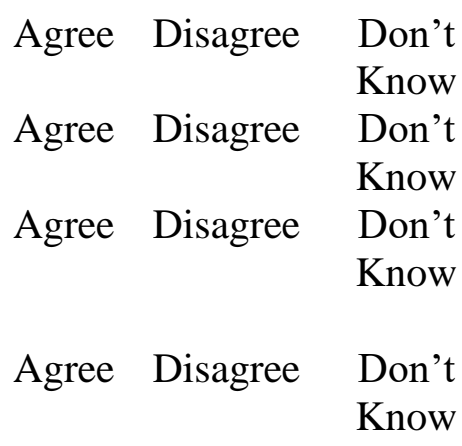

Agree Disagree Don't

Know

Agree Disagree Don't

Know

Agree Disagree Don't

Know

Agree Disagree Don't

Know

Agree Disagree Don't

Know 
28. The relationship or connection between an individual and the research staff/research

Agree Disagree Don't organization.

29. Whether the study seems invasive or risky.

Agree Disagree Don't

Know

Please write any additional comments here.

Thank you for your responses! By completing this survey, you are helping researchers understand what helps or gets in the way of the decisions made by a person living with memory loss to participate (or not) in clinical research. 